\title{
Les langues savantes
}

\section{Xavier Lafon (dir.)}




\section{Les langues savantes}

\section{Xavier Lafon (dir.)}

DOI : 10.4000/books.cths.441

Éditeur : Éditions du Comité des travaux historiques et scientifiques Année d'édition : 2017

Date de mise en ligne : 13 novembre 2018

Collection : Actes des congrès nationaux des sociétés historiques et scientifiques

ISBN électronique : 9782735508686

\section{Sbooks}

http://books.openedition.org

\section{Édition imprimée}

Nombre de pages : 117

\section{Référence électronique}

LAFON, Xavier (dir.). Les langues savantes. Nouvelle édition [en ligne]. Paris : Éditions du Comité des

travaux historiques et scientifiques, 2017 (généré le 22 décembre 2020). Disponible sur Internet: <http://books.openedition.org/cths/441>. ISBN : 9782735508686 . DOI : https://doi.org/10.4000/ books.cths. 441 .

(C) Éditions du Comité des travaux historiques et scientifiques, 2017

Conditions d'utilisation:

http://www.openedition.org/6540 
COMITÉ DES TRAVAUX HISTORIQUES ET SCIENTIFIQUES

\section{Les langues savantes}

Sous la direction de

Xavier LAFON

ÉDITIONS DU CTHS

2017 
Ministère de l'Éducation nationale, de l'Enseignement supérieur et de la Recherche

Congrès national des sociétés historiques et scientifiques 139 , Nîmes, 2014

Collection Actes des congrès des sociétés historiques et scientifiques, Version électronique

ISSN 1764-7355 


\section{Introduction}

Xavier LAFON

p. 5

Dimensions linguistique et prosodique de l'ironie chez le chroniqueur humoristique radiophonique Élodie BAKLOUTI \& Fabrice HIRSCH

La communication technique interne chez Peugeot:

les journaux d'entreprise

Robert BELOT \& Pierre LAMARD

Progrès et ruptures de la communication dans les sciences exactes Jean BRINI

Nommer le travail agricole. Application à la viticulture (1850-1950) Jean-Louis ESCUDIER

Joseph-Diez Gergonne, ses Annales de Mathématiques éditées à Nîmes, et le premier article d'Évariste Galois Jean-Michel FAIDIT

Les mots pour démolir : les instruments discursifs et les schèmes cognitifs de l'action publique sur et par l'espace

Les métaphores favorisent-elles la compréhension d'une théorie scientifique? Le cas des images darwiniennes de "lutte pour la vie» et de " sélection naturelle » 
Ya-t-il une langue de l'économie?

Jean MATOUK

p. 94

Professionnels et professionnelles de la publicité française:

les femmes dans l'histoire de la profession publicitaire

de 1925 à nos jours

Nathalie PELIER

p. 98

L'explication du geste technique, la transmission

des savoir-faire : l'indicible et la communication non verbale

Bénédicte ROLLAND-VILLEMOT

p. 107 


\title{
Les langues savantes
}

\author{
Xavier LAFON \\ Professeur d'archéologie romaine à l'université d'Aix-Marseille, \\ ancien directeur de l'institut de recherche sur l'architecture antique, USR 3155
}

Extrait de : Xavier LAFON, Les langues savantes, Paris, Édition électronique du CTHS (Actes des congrès des sociétés historiques et scientifiques), 2017.

Cet article a été validé par le comité de lecture des Éditions du CTHS dans le cadre de la publication des actes du $139^{\mathrm{e}}$ Congrès national des sociétés historiques et scientifiques tenu à Nîmes en 2014.

La communication et les langages qui la soutiennent ne concernent pas uniquement les domaines littéraire, politique ou religieux. Une session de ce congrès consacré aux langages et à la communication devait donc comporter une section consacrée aux domaines plus techniques, des sciences «dures» aux métiers, de l'élaboration à la transmission des savoirs. Les différents aspects abordés dans ces dix communications sont par définition multiples et semblent à première vue ne présenter aucune unité, aucun point commun, de l'abstraction totale des mathématiques au geste infiniment répété de la potière. À y regarder de plus près, ces «communications » présentent cependant des éléments de convergence non négligeables.

La langue «commune» utilisée tous les jours pour communiquer avec ses proches ne permet pas de formuler toute la palette de nuances et même de contenus exigés pour rendre compte d'un domaine particulier de savoir, qu'il soit scientifique ou qu'il relève de travaux appliqués, en particulier dans le cadre du travail artisanal. Il faut donc créer et partager une langue spécifique, au moins un vocabulaire propre. C'est ainsi que l'on peut comprendre (J.-L. Escudier) le maintien encore aujourd'hui dans les zones de vignobles français d'un vocabulaire spécifique à la viticulture permettant par exemple de mesurer le temps de travail comme la spécificité des actions à entreprendre, les types de relation entre propriétaire et exploitant liés en grande partie au caractère pluriannuel de la vigne. Emprunté pour l'essentiel aux différents «patois », ce vocabulaire traduit l'unité de la profession et son histoire complexe par des mots divers selon les régions, mais convergents. En contrepartie, il nécessite une véritable initiation pour ceux amenés à le pratiquer (qui peut être «naturelle» pour ceux qui naissent et grandissent dans ce milieu) alors que ce langage demeure pratiquement totalement hermétique aux autres. Mutatis mutandis, cette situation n'est pas très différente de celle de l'anglais utilisé pour rendre compte des concepts liés à l'économie et plus spécifiquement à la finance (J. Matouk). Ces mots détachés du contexte linguistique qui les a vu naître, en l'occurrence l'anglais, prennent donc une valeur que l'on peut qualifier d'universelle tout en devenant les outils d'un groupe plus ou moins étendu d'individus, y compris parmi les anglophones. Il n'est pas évident que tous comprennent le sens économique particulier donné à ce vocabulaire.

Le même besoin de dépasser un cadre linguistique trop restreint caractérise le domaine scientifique: aucune science ne peut être considérée comme autonome sans l'appropriation d'un champ spécifique, d'une méthodologie particulière mais également d'un langage adapté. Il peut s'agir cette fois de véritables concepts même s'ils demeurent rassemblés sous un vocable unique. Là encore, la spécificité de chaque discipline scientifique la rend quelque peu hermétique aux autres. Dans l'article sur les destructions urbaines contemporaines (P. Genestier \& C. Jacquenod-Desforges), il suffit de voir la nécessité pour la linguiste de donner dans les notes un véritable lexique : l'intrusion de cette science, la linguistique ou du moins l'étude des discours officiels, sur un sujet où 
l'axe dominant est l'urbanisme, crée un important porte-à-faux, et pour prendre en compte puis se faire comprendre des premiers intéressés, les "urbanistes » au sens large comprenant donc également les politiques, il n'existe pas d'autre moyen que de préciser le sens des concepts utilisés dans une perspective éminemment scientifique...

Des raisons historiques sont à l'origine de ces vocabulaires spécialisés. Il n'est pas question de les détailler ici, mais leur adaptation et leur diffusion dans un milieu plus ou moins large posent d'autres séries de questions: comment, tout simplement, communiquer en interne avec les spécialistes, et en externe pour faire partager ces connaissances? La question de la diffusion de ces langages spécifiques et donc de leur communication permet de repérer d'autres points de convergence possible entre ces différentes interventions.

Certains outils utilisés pour communiquer peuvent relever de pratiques rhétoriques comme la polysémie en mettant en avant un sens du mot différent de celui le plus habituellement retenu, ou en multipliant les métaphores pour tenter de faire vivre et partager un nouveau concept. À ce sujet la compréhension par ses contemporains des propos de Darwin (A. La Vergata), permet de vérifier, en particulier par le biais des traductions, combien il a fallu de temps pour que ses concepts les plus novateurs puissent être compris et assimilés. Il y a donc bien un risque d'approximation ou d'erreur à partir d'une communication qui se veut pourtant totalement rationnelle et scientifique (J. Brini). Il existe également des moyens beaucoup plus complexes pour communiquer les savoirs accumulés, avec l'espoir de les augmenter grâce aux interventions de toute la communauté en favorisant les échanges. Une revue scientifique comme les Annales de mathématiques (J.-M. Faidit) semble un outil adéquat, même si ses moyens demeurent très modestes. En l'occurrence, il s'agit d'une initiative individuelle mais qui est largement adoptée par la communauté scientifique concernée, pourtant encore en voie de constitution, et de toute évidence beaucoup moins nombreuse qu'aujourd'hui, parmi laquelle la sélection d'articles demeure beaucoup plus aisée, permettant à un jeune étudiant comme Évariste Gallois d'être publié au même titre qu'un savant confirmé. Dans ce souci de partage et de diffusion des connaissances, une entreprise industrielle comme Peugeot (P. Lamard, R. Belot), multiplie tout au long de son histoire les supports pour faire partager les progrès des connaissances, tout en favorisant «l'esprit maison », avec des hiérarchies clairement définies dans les publics visés, selon les niveaux et les postes occupés. Dans ce cas, les mots ne suffisent plus et il faut mettre en jeu d'autres supports, notamment les «images ». Ces dernières peuvent être de nature très diverses et dans le cas de Peugeot nécessiter l'intervention de spécialistes, photographes ou cinéastes professionnels. Mais l'enquête ethnographique sur les métiers de l'artisanat (RollandVillemot) montre que l'ethnologue doit savoir non seulement décrire les gestes du métier qu'il observe (en suivant une matrice très codifiée dans son organisation) mais également dessiner: le proverbe dit qu'un dessin vaut mieux qu'un long discours, il existe des situations où le discours n'est tout simplement pas possible! Dans d'autres cas le contenu réel du message peut se révéler sans dessin ou photographie complémentaire en prenant en compte, au-delà des mots prononcés (É. Baklouti), l'intonation, le rythme du phrasé, les aspects les plus physiologiques de la parole: il est donc possible de montrer scientifiquement, c'est-à-dire preuves à l'appui, que la même phrase peut traduire des interprétations fondamentalement différentes.

La communication ou plutôt le désir de convaincre à tout prix, au-delà de la preuve scientifique (et souvent contre cette dernière) peut aller jusqu'à prendre la forme de la "réclame», la publicité d'aujourd'hui. La réputation de ce média est demeurée longtemps très négative $(\mathrm{N}$. Pellier) rendant difficile l'étude de leurs auteurs et encore plus de leurs auteures car les noms d'emprunt sont la règle pour ceux qui sont les créatifs dans les agences. De fait certains comme Peugeot font mine de s'en passer au profit de la seule information sur les « produits ». La frontière n'est donc pas toujours sensible entre désir d'informer et désir de convaincre... 


\title{
Dimensions linguistique et prosodique de l'ironie chez le chroniqueur humoristique radiophonique
}

\author{
Élodie BAKLOUTI, \\ Fabrice HIRSCH \\ Université Paul Valéry, UMR 5267 Praxiling, CNRS
}

Extrait de : Xavier LAFON, Les langues savantes, Paris, Édition électronique du CTHS (Actes des congrès des sociétés historiques et scientifiques), 2017.

Cet article a été validé par le comité de lecture des Éditions du CTHS dans le cadre de la publication des actes du $139^{\mathrm{e}}$ Congrès national des sociétés historiques et scientifiques tenu à Nîmes en 2014.

\section{État de l'art}

\section{La prosodie}

La prosodie se définit généralement comme l'étude du rythme et de l'intonation dans la parole. En d'autres termes, une étude prosodique est susceptible de porter sur tous les éléments suprasegmentaux présents dans un signal de parole. La durée des segments, qui impacte le débit de la parole, la fréquence fondamentale, responsable de la sensation de hauteur, l'intensité, procurant la sensation de puissance vocale, sont les paramètres les plus généralement utilisés en vue de décrire les variations de cadences et de mélodie dans un énoncé (Lacheret, 2011, 28).

Plusieurs fonctions sont généralement attribuées à la prosodie (Martinet ${ }^{1}, 1960$, 108) : celle-ci permet notamment de distinguer une phrase affirmative $d^{\prime}$ une phrase interrogative, raison pour laquelle il est possible de parler de fonction distinctive. Le rythme et l'intonation peuvent également être utiles en vue de hiérarchiser syntaxiquement un énoncé ; on parlera alors de fonction démarcative. Enfin, la prosodie peut avoir une fonction expressive, dans la mesure où elle permet de laisser transparaître une émotion ou encore de conférer une connotation à un énoncé (Léon, 1993, 38). Par conséquent, il est permis de penser qu'un sens secondaire donné à un énoncé peut être marqué d'un point de vue rythmique et/ou intonatif.

\section{L'ironie}

D’un point de vue rhétorique, l'ironie peut se définir comme un «énoncé par lequel le locuteur dit le contraire de ce qu'il veut faire entendre » (Fontanier, 1977, 145). Dans le cadre de notre article, qui porte en partie sur des corpus médiatiques, on parlera plus spécifiquement d'ironie pour désigner un écho implicite qui est fait à un énoncé dont le locuteur veut marquer le manque de pertinence, voire l'absurdité :

«Le locuteur fait écho à une proposition d'une manière propre à manifester qu'il la désapprouve soit parce qu'elle manque de vérité (et partant de pertinence), soit parce qu'elle manque directement de pertinence. » (Sperber \& Wilson, 1978, 409)

C'est à partir de ces définitions que nous avons pu sélectionner des passages ironiques dans les chroniques humoristiques d'une part et, d'autre part, former des énoncés ironiques pour la constitution du corpus dit «naturel ». De nombreux linguistes partent

1. A. Martinet, 1960, p. 108. 
du principe que l'ironie est marquée prosodiquement (Kebrat-Orecchioni 1980, 110, Eggs $2005,4)$. Cette affirmation repose pourtant uniquement sur une appréhension empirique de l'ironie. À l'heure actuelle, aucune étude ne démontre formellement que le locuteur marque son ironie par des signaux prosodiques.

Partant du fait que l'ironie s'énonce de manière indirecte, on peut postuler que des éléments non-verbaux, dont la prosodie, participent à sa reconnaissance, raison pour laquelle notre étude vise à analyser la relation entre le rythme et l'intonation d'une part et la réalisation linguistique de l'ironie d'autre part. Anne Karpf évoque justement le fait que trop peu d'études linguistiques prennent en considération cette dimension prosodique ou "vocale » dans l'étude de la production de sens des énoncés, la mise en lien des plans linguistique et prosodique étant pourtant essentielle dans le processus de la communication humaine :

«La plupart des études linguistiques sur la conversation laissent de côté le véhicule qui la rend possible. Voix et discours sont traités à l'identique, et le discours l'est à peine davantage qu'un simple langage parlé. Le langage parlé est considéré comme porteur essentiel du sens, comme si la voix n'était que le véhicule des mots, la force qui gouverne la direction et la vitesse d'une phrase. Nous décortiquons le langage pour en débusquer le sens sémantique, puis nous jetons la voix comme nous nous débarrasserions d'un détritus. » (Karpf, 2008, 245)

\section{Objectif et hypothèse}

Le premier objectif de ce travail sera de vérifier l'existence d'une "prosodie de l'ironie» en parole dite «normale» et en parole radiophonique. En d'autres termes, nous nous demanderons s'il existe une prosodie de l'ironie. Si cette dernière est avérée, nous chercherons à vérifier si elle se manifeste de façon est similaire en parole dite " naturelle » et dans les médias. La première hypothèse que nous souhaitons examiner dans le cadre de cet article est celle selon laquelle les locuteurs pourraient s'aider d'une intonation particulière pour transmettre l'ironie, notamment lorsqu'un énoncé est ambigu. Le rythme et l'intonation aideraient ainsi à véhiculer un sens supplémentaire en direction de l'interlocuteur.

Le second postulat que nous éprouverons concerne les patterns prosodiques. Il s'agira de vérifier si ces derniers, éventuellement attestés en parole "normale ", seraient différents de ceux potentiellement relevés en parole radiophonique, dans la mesure où une mise en scène du discours s'instaure dans ce cadre précis.

\section{Méthodologie}

\section{Les corpus}

Le corpus en parole dite "normale »

10 locuteurs (5 hommes et 5 femmes) ont été enregistrés dans une chambre insonorisée en train de prononcer 10 phrases sur un ton neutre puis sur un ton ironique. Pour chaque phrase, un contexte était initialement présenté par écrit. Ce contexte est constitué de façon à faire émerger chez un locuteur soit une phrase prononcée sur un ton neutre (en a) soit une phrase prononcée sur un ton ironique (en b), comme il est possible de le constater dans le cas suivant :

(a) Paul et Martin finissent leur café lorsqu'une jolie serveuse vient leur demander s'ils souhaitent prendre autre chose. Ils répondent négativement et Paul en la regardant s'éloigner admiratif dit à Martin :

- Elle est vraiment belle. 
(b) Paul et Martin sont venus s'asseoir à la terrasse d'un café dans lequel Paul a croisé une serveuse qu'il a trouvée superbe, ce qu'il n'a pas manqué de dire à Martin. Alors qu'ils se sont rendus dans ce café afin que Martin constate la beauté de celle-ci, une serveuse aux traits disgracieux vient leur demander s'ils souhaitent prendre quelque chose. Ils répondent négativement et Martin en la regardant s'éloigner, s'adresse à Paul ironiquement :

- Elle est vraiment belle!

Une fois le corpus enregistré, un test de perception, nécessitant l'utilisation du logiciel Perceval, a été réalisé auprès de 10 auditeurs afin de vérifier que les phrases qui devaient être prononcées sur un ton ironique sont catégorisées de la sorte par un jury naïf. Seules les phrases perçues comme étant ironiques ont fait l'objet de notre étude.

\section{Le corpus radiophonique}

Les chroniques radiophoniques de 3 humoristes français (Sophia Aram, François Morel et Stéphane Guillon) ont été reprises d'Internet. 20 extraits qualifiés d'ironiques ont été identifiés et analysés.

\section{Analyse prosodique}

Les phrases du corpus de parole dite "normale» et celles provenant des chroniqueurs ont été analysées à l'aide du logiciel Praat et de Prosogram. Le premier programme cité a permis notamment de relever les éventuels ajouts et de calculer le débit moyen par phrase tandis que Prosogram a été employé en vue d'obtenir un suivi de la fréquence fondamentale (valeurs données en demi-tons).

\section{Principaux Résultats}

\section{Résultats en parole dite «normale»}

Les figures 1 et 2 présentent la phrase «Tu es en avance » prononcée par le locuteur Michel, respectivement sur un ton neutre et sur un ton ironique. Comme il est possible de le constater, la phrase de la figure 1 est produite sur un ton relativement plat avec une baisse de la fréquence fondamentale, correspondant à l'accent final du français, sur la dernière syllabe. Aucune proéminence n'est visible ici. Concernant la même phrase produite sur un ton ironique, on observe un pic de F0 sur la première syllabe du nom «avance » qui constitue l'élément rhématique de l'énoncé. S'en suit une baisse de la fréquence fondamentale sur la dernière syllabe du même mot.

Notons que ce schéma, consistant à observer une proéminence sur l'élément rhématique d'un énoncé produit sur le ton ironique, est celui qui est revenu le plus régulièrement dans notre corpus.

Par ailleurs, lorsque l'élément rhématique est accompagné d'un adverbe modalisateur, on remarque un déplacement de l'accent sur ce même adverbe. C'est le cas pour les figures 3 et 4 qui représentent la phrase «Elle est vraiment belle » produite par le même locuteur à nouveau sur un ton neutre, puis sur un ton ironique. $\mathrm{Si}$, dans le premier cas, on relève à nouveau une intonation plate se terminant par une baisse de F0 sur la dernière syllabe, la phrase produite ironiquement se caractérise par une montée de la fréquence fondamentale sur l'adverbe "vraiment» avant qu'une diminution de la fréquence fondamentale ne soit réalisée sur la dernière syllabe de l'énoncé.

D'autres particularités ont également été notées concernant le ton ironique. C'est le cas notamment pour la phrase «Elle est vraiment belle » prononcée par la locutrice Christel (figure 5). En effet, s'il est possible de relever une courbe intonative relativement plate tout au long de la production de cette phrase, celle-ci est accompagnée d'une voix rieuse, en témoigne le bruit de friction présent sur certains segments (voir flèches blanches sur la figure 5). 
De même, l'ironie peut également être exprimée à l'aide d'un rythme saccadé. C'est le cas pour la phrase «Ton bureau est super bien rangé » produite par la locutrice Maud. En effet, si aucune proéminence n'est observable sur cet énoncé (figure 6), on remarque un allongement de la durée de l'ensemble des voyelles présentes dans ce même énoncé (tableau 1).

Enfin, signalons également que, dans $36 \%$ des cas, nos locuteurs ont ajouté le marqueur «hein » à la phrase à prononcer sur un ton ironique. C'est le cas par exemple pour l'énoncé «Tu es en avance » produit par la locutrice Nada (voir figure 7) prononcée avec une montée de F0 sur la dernière syllabe du mot «avance », montée qui se prolonge sur le mot « hein » situé en fin de phrase.

Les travaux menés dans la littérature sur le sujet révèlent d'ailleurs qu'il s'agit d'un marqueur discursif indiquant la relation phatique, la recherche de la connexion avec l'autre (Malandain, 1983, 83 ; Traverso 1996, 66). En d'autres termes, le locuteur appelle à la perception de l'autre sur l'énoncé qu'il vient de produire, perception que le locuteur veut co-orienter à la sienne.

\section{Résultats en parole radiophonique}

Le procédé consistant à accentuer l'adjectif sur lequel repose l'ironie est également employé en parole radiophonique. C'est le cas par exemple pour Stéphane Guillon dans l'énoncé "Elle est classe la couverture de Paris Match». L'ironie est repérable à la discordance qui existe entre l'adjectif « classe » subjectif axiologique positif se rapportant ici, en tant qu'attribut, au sujet "elle», renvoyant à la couverture du magazine représentant l'ancienne prostituée Zaïa. La suite du discours de l'humoriste dans lequel il décrit l'ancienne prostituée comme adoptant une position lascive qui met en valeur sa "croupe» et sa poitrine généreuse, les connaissances partagées autour de la catégorisation en tant que prostituée qu'il utilise, nous invitent à comprendre qu'il ne pense pas que cette couverture est effectivement «classe» mais vulgaire. Une discordance de l'ordre de l'inversion s'instaure donc entre le pensé et le dit de l'humoriste.

C'est le même type de discordance qui se réalise dans l'énoncé de Sophia Aram lorsqu'elle évoque $l^{\prime}$ «idée lumineuse de la bien nommée Christine Maso ». En effet, l'adjectif subjectif axiologique positif «lumineuse» entre en discordance avec le terme " maso » qui sert à la fois à dénommer et à décrire la personne qui a émise cette idée. L'humoriste suggère en effet un lien motivé et non arbitraire entre le sens de dénomination de la personne "Maso » et le caractère de cette dernière, femme politique dont l'humoriste ne partage pas les opinions, en utilisant la boucle méta énonciative «bien nommée » signalant l'adéquation du nom «maso » au référent réellement perçu. Dans ces deux énoncés antiphrastiques, on observe une proéminence respectivement sur les adjectifs "classe » (figure 8) et "lumineuse », appartenant aux adjectifs subjectifs axiologiques positifs dans le contexte (figure 9).

On notera cependant que, contrairement à la parole dite "normale », très peu d'ajouts dans les chroniques humoristiques ont été relevés. Aucun marqueur de type « hein » n'a par exemple été répertorié. Les seules adjonctions présentes dans les énoncés radiophoniques ironiques sont davantage réalisées dans le but de produire un effet d'oralité et de mettre en scène le discours radiophonique. 


\section{Synthèse, limites et perspectives}

\section{Synthèse}

En résumé, il est possible de constater la présence d'un accent d'insistance sur les éléments axiologiques lorsqu'un énoncé est ironique. Dans le cas où un énoncé contiendrait un adverbe intensif (comme "vraiment», "super», etc.) modifiant un adjectif subjectif axiologique, la proéminence porte sur cet adverbe. D'autres particularités ont été observées pour mettre en évidence l'ironie, comme l'utilisation d'une voix rieuse, d'un rythme saccadé ou d'un marqueur discursif de demande d'approbation de type « hein », «n'est-ce pas » ou « dis donc ».

Dans le cadre du discours radiophonique, on remarque également la présence d'un accent soit sur les éléments axiologiques eux-mêmes, soit sur les adverbes intensifs les accompagnant. En revanche, la demande d'approbation est moins équivoque puisque l'on retrouve moins d'ajouts. Cela peut sans doute s'expliquer par le fait, d'une part, que le discours radiophonique est davantage formaté et, d'autre part, que son intonation dépend en grande partie de la mise en scène humoristique.

\section{Limites}

La principale limite à ce travail porte sur le caractère «naturel» de la parole dite « normale ». En effet, le fait de faire lire une phrase à nos locuteurs en imposant plus ou moins un ton neutre puis un ton ironique rend l'exercice quelque peu artificiel. Par ailleurs, «imposer » une intonation ironique suppose que le locuteur produise un énoncé qui sera tout sauf neutre. Par conséquent, cela écarte la possibilité de trouver des énoncés ironiques produits sur un ton neutre alors même que ceux-ci doivent bel et bien exister. En outre, notre étude ne tient pas compte des éléments extra-linguistiques comme les gestes, le regard, etc. qui peuvent accompagner l'ironie.

\section{Perspectives}

Il serait intéressant de reprendre cette étude avec davantage de locuteurs et de phrases à prononcer sur un ton ironique. En outre, il serait également important d'augmenter le nombre d'occurrences ironiques prononcées par des chroniqueurs humoristiques pour compléter notre analyse. Enfin, le lien entre la manière de marquer l'ironie d'un point de vue prosodique et le type d'ironie mériterait également d'être étudié. En effet, selon les théories énonciativistes (Benveniste, 1966, 156 ; Kebrat, 1980, 114), un locuteur peut s'impliquer plus ou moins dans son discours par l'usage de termes objectifs n'impliquant pas la notion d'appréciation ou de termes subjectifs qui, au contraire, comportent l'idée d'une évaluation. Un locuteur pouvant utiliser ces diverses catégories d'unités dans l'ironie, on peut supposer l'existence d'énoncés ironiques de différentes natures.

Pour exemple, si un énonciateur, regardant la pluie tomber, prononce la phrase « le soleil brille ce matin », nous aurons à faire à un énoncé ironique objectif. Dans le cas où une locutrice dirait «cet enseignant est mignon » tout en pensant le contraire, il s'agirait d'un énoncé ironique subjectif. Partant de là ainsi que du postulat selon lequel la prosodie rend plus évidente la reconnaissance de l'ironie, nous voudrions vérifier ou infirmer notre hypothèse selon laquelle plus un énoncé est subjectif, plus l'ironie sera marquée prosodiquement et inversement, dans la mesure où la perception du caractère ironique sera plus aisée pour l'interlocuteur dans le cas où le fait énoncé porte sur une réalité unanimement vérifiable. 


\begin{abstract}
Résumé
Mise en parallèle de la réalisation prosodique de l'ironie chez le chroniqueur humoristique et en parole naturelle. Se situant à la fois dans la catégorie des professionnels de l'écrit et dans celle des professionnels de l'oral, le chroniqueur humoristique radiophonique peut être considéré comme l'un des successeurs du poète comique du monde antique. Pour susciter le rire, le chansonnier dispose d'une multitude de procédés comme la caricature, la parodie, le jeu de mot... Dans le cadre de cet article, nous voulons nous interroger sur ce que nous considérons avec Charaudeau (2011) comme un autre de ces procédés: l'ironie. En particulier, nous voulons questionner le lien qui peut exister entre la prosodie et la réalisation linguistique de l'ironie. Pour ce faire, nous comparons des extraits ironiques issus de chroniques humoristiques avec des énoncés ironiques prononcés par des locuteurs en contexte dit «naturel» et tentons de vérifier si l'ironie est marquée prosodiquement et, si oui, si ces manifestations prosodiques sont les mêmes dans les deux contextes.
\end{abstract}

\title{
Bibliographie
}

BENVENISTE Emile. 1966, Problèmes de linguistique générale, tome 1, Paris, Gallimard.

EGGS Ekkehard. 2009, Rhétorique et argumentation: de l'ironie. Argumentation et analyse de discours, 2, 1-15.

FONTANIER Pierre. 1977, Les figures du discours, Paris, Flammarion.

Kerbrat-OrecChioni Catherine. 1980, L'ironie comme trope, Poétique, 41, 108-127.

KeRBRAT-ORECCHIONi Catherine. 1980, L'énonciation de la subjective dans le langage, Paris, Armand Colin.

KARPF Anne. 2008, La voix: un univers invisible, traduit de l'anglais par Geneviève Brzustowski, Paris, Éditions Autrement.

LACHERET Anne 2011, Le corps en voix ou l'expression prosodique des émotions, Évolutions psychomotrices, 23, 90, 25-37.

LÉON Pierre. 1993, Précis de phonostylistique, parole et expressivité, Paris, Nathan.

MALANDAIN, Jean-Louis. 1983, Il faut bien faire avec des bribes hein! Français dans le monde, $176,84-86$

MARTINET André. 1960, Éléments de linguistique générale, Paris, Armand Colin.

SPERBER Dan \& Wilson Deirdre. 1978, Les ironies comme mention. Poétique, 36, 399-412.

TRAVERSO Véronique, 1996, La conversation familière: analyse pragmatique des interactions, Lyon, Presses Universitaires de Lyon, coll. Linguistique et sémiologie. 
Annexe

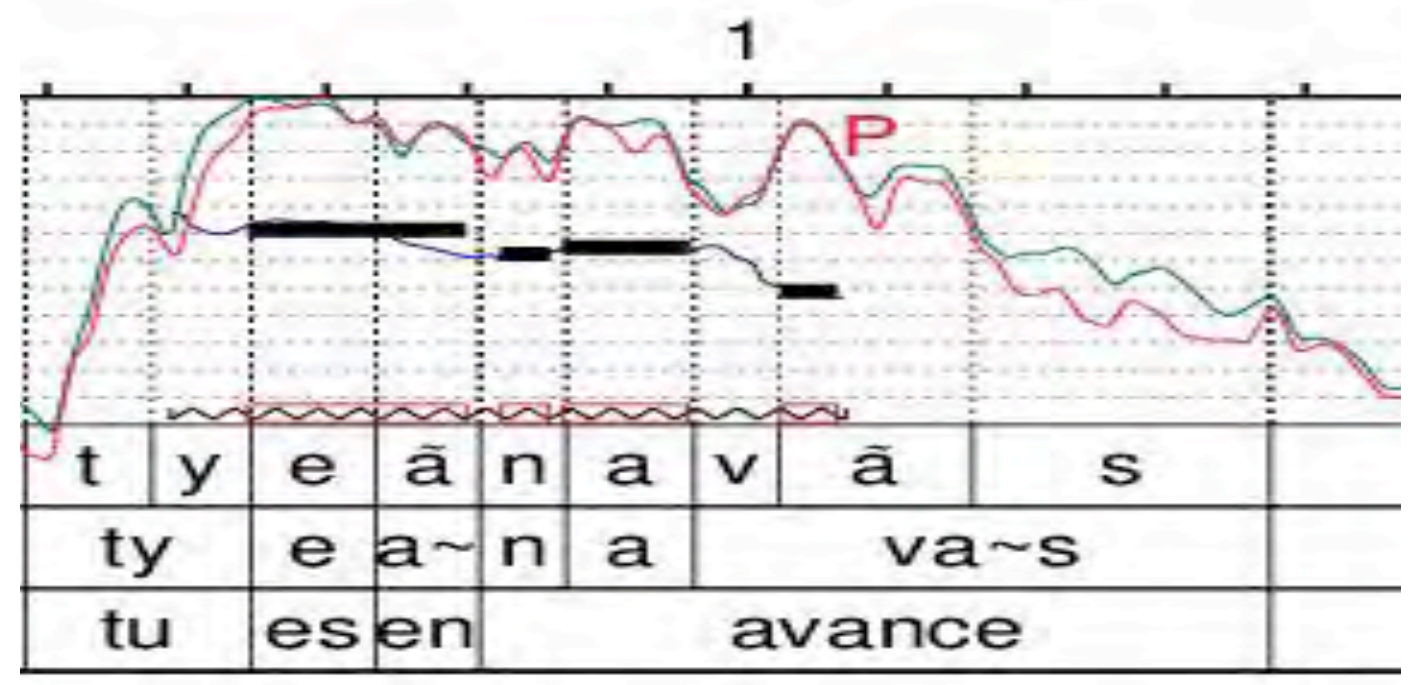

Figure $1:$ : Tu es en avance » prononcé sur un ton neutre.

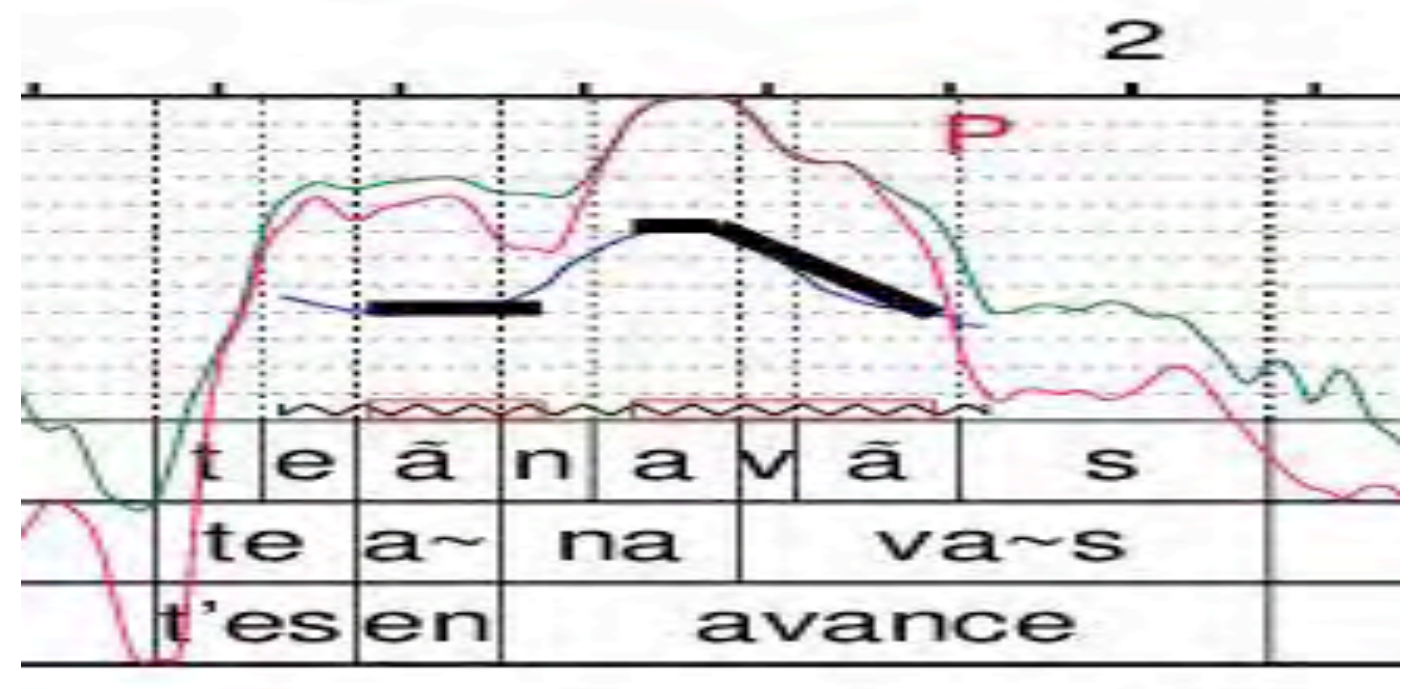

Figure 2 : «Tu es en avance » prononcé sur un ton ironique (?). 


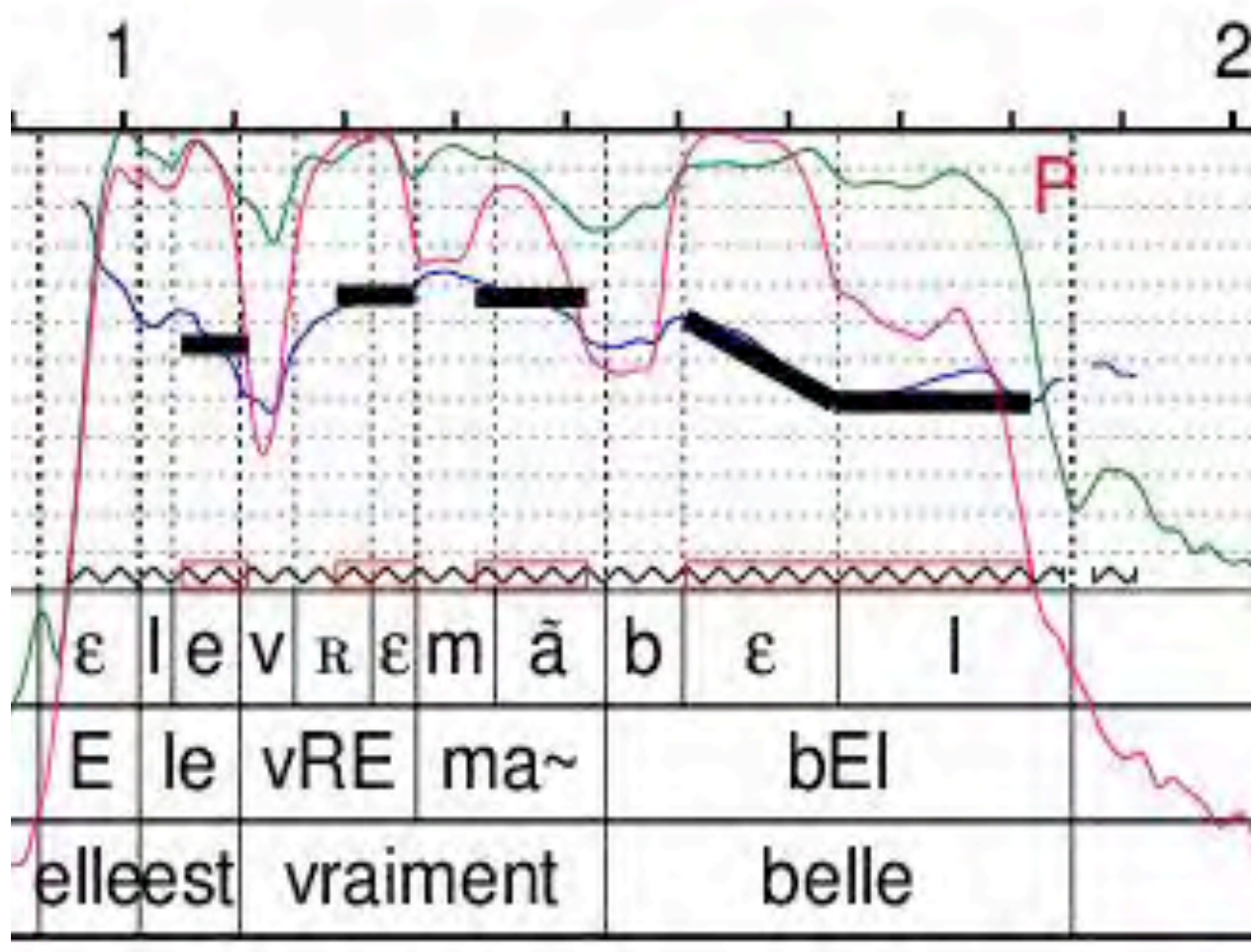

Figure $3:$ « Elle est vraiment belle » prononcé sur un ton neutre.

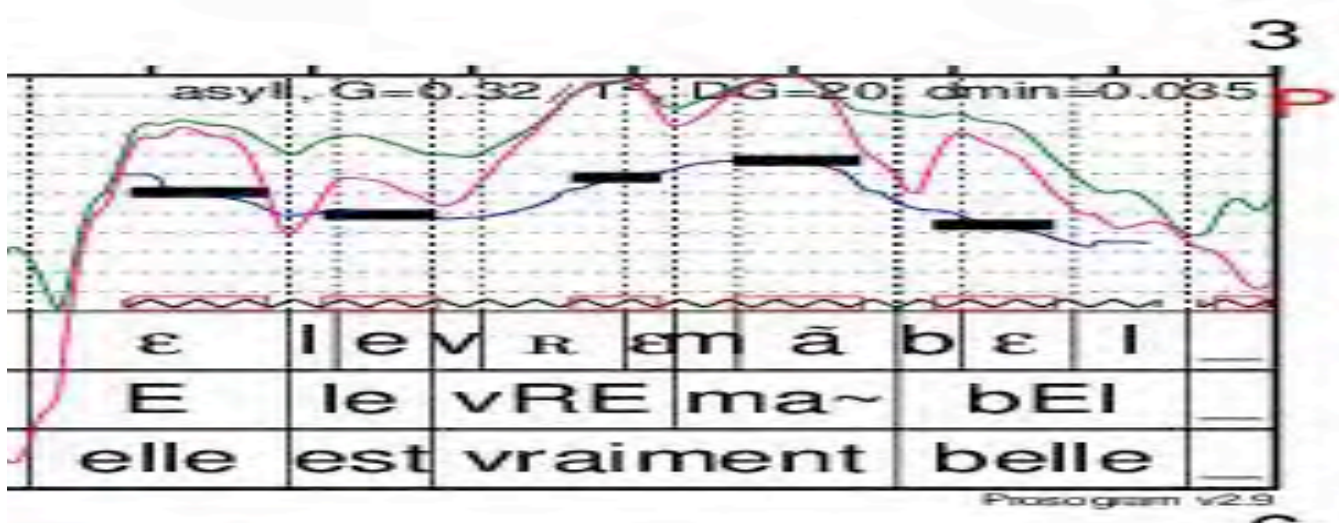

Figure 4 : « Elle est vraiment belle » prononcé sur un ton ironique (?). 


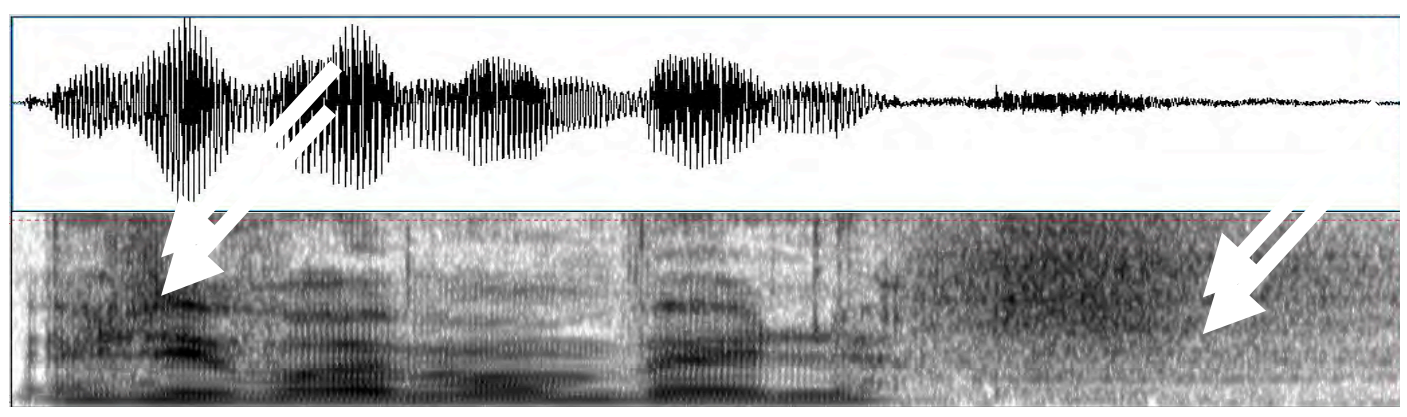

Figure 5: Suivi de F0, signal acoustique et spectrogramme de la phrase «Elle est vraiment belle » produite sur un ton ironique par Christel. Les flèches indiquent la présence d'un bruit de friction.

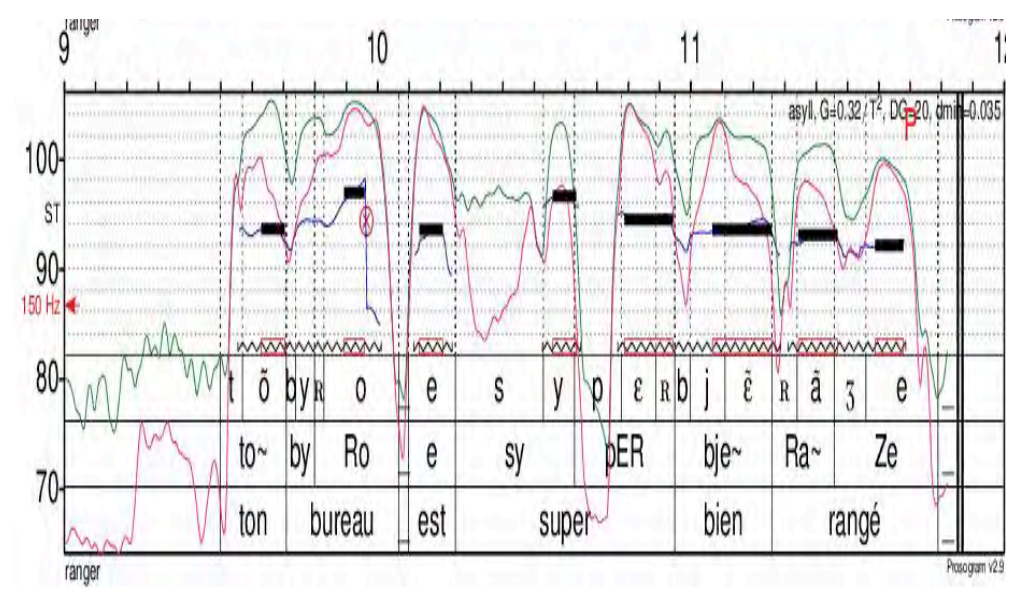

Figure 6: courbe intonative de la phrase «Ton bureau est super bien rangé » prononcée par la locutrice $\mathrm{M}$ sur un ton ironique.

\begin{tabular}{|c|c|c|}
\hline Voyelles & Normal & Ironie \\
\hline $\mathrm{o} \sim$ & 110 & 140 \\
\hline $\mathrm{y}$ & 90 & 60 \\
\hline $\mathrm{o}$ & 130 & 240 \\
\hline $\mathrm{e}$ & 159 & 149 \\
\hline $\mathrm{y}$ & 90 & 100 \\
\hline $\mathrm{E}$ & 80 & 110 \\
\hline $\mathrm{E} \sim$ & 70 & 150 \\
\hline $\mathrm{a} \sim$ & 130 & 120 \\
\hline $\mathrm{e}$ & 140 & 230 \\
\hline Moyenne & $\mathbf{1 1 1}$ & $\mathbf{1 4 4}$ \\
\hline E-T & $\mathbf{3 0}$ & $\mathbf{5 8}$ \\
\hline
\end{tabular}

Tableau 1: durée moyenne des voyelles dans la phrase «Ton bureau est super bien rangé » prononcée par la locutrice M sur un ton ironique 


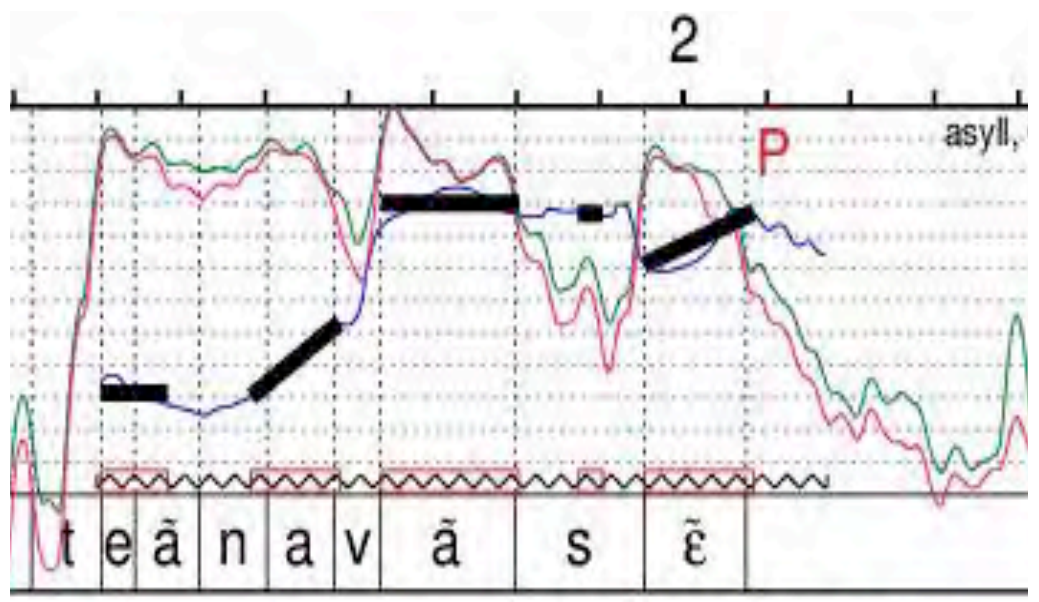

Figure 7 : courbe intonative de la phrase «Tu es en avance » prononcée par la locutrice Nada sur un ton ironique.

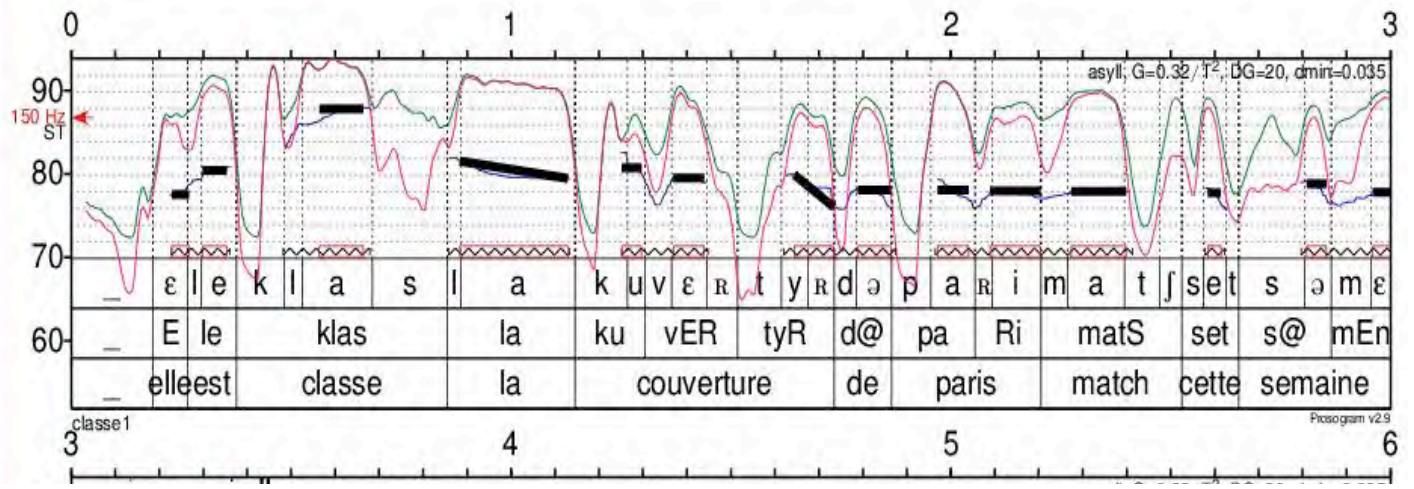

Figure 8: Contour intonatif de l'énoncé « elle est classe la couverture de Paris Match cette semaine » prononcé par Stéphane Guillon.
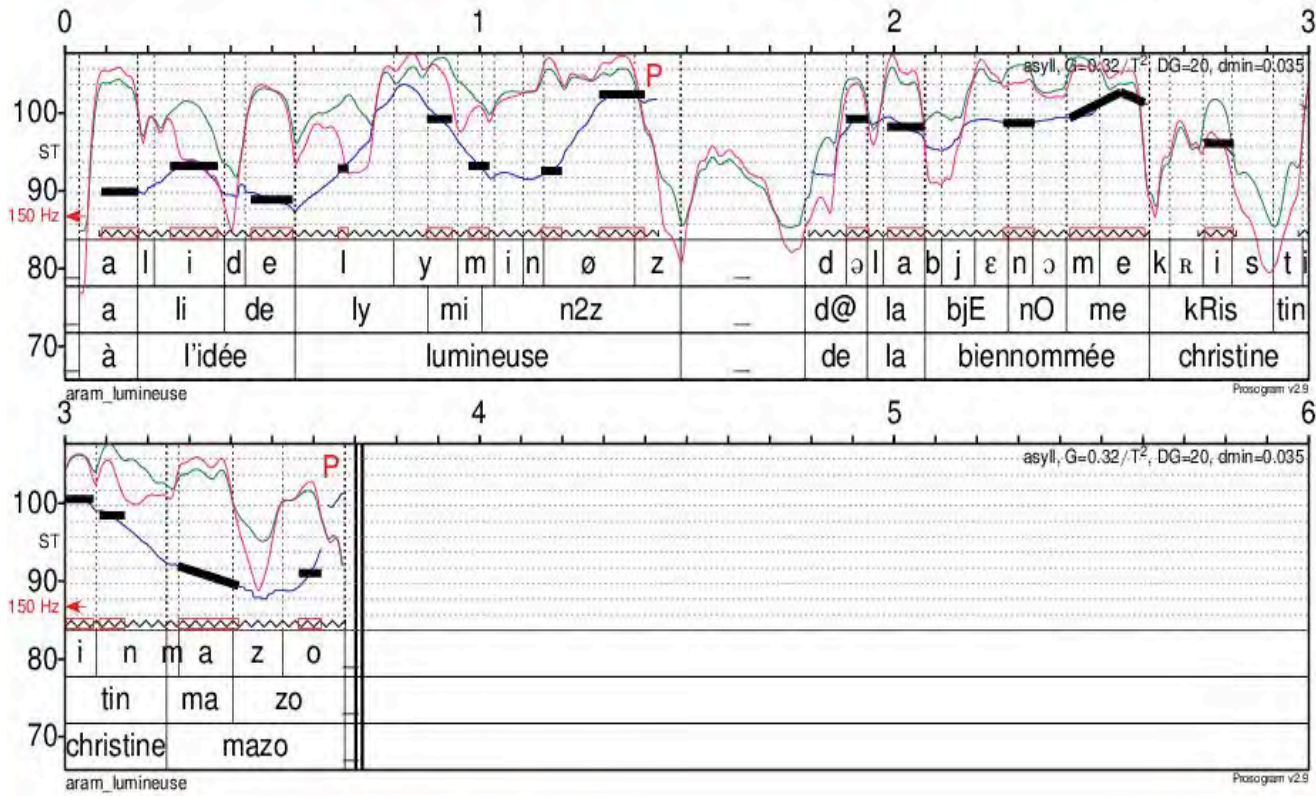

Figure 9: Contour intonatif du syntagme prépositionnel «à l'idée lumineuse de la bien nommé Christine Mazo » prononcé par Sophia Aram. 


\title{
La fabrique du discours de l'entreprise : les vertus heuristiques de la communication technique de Peugeot
}

\author{
Robert BELOT \\ Professeur des Universités \\ Université de Lyon-Saint-Étienne
}

UMR EVS 5600 CNRS

Pierre LAMARD

Professeur des Universités

Université de Belfort-Montbéliard UMR FEMTO-RECITS 6174 CNRS

\author{
Extrait de : Xavier LAFON, Les langues savantes, Paris, \\ Édition électronique du CTHS (Actes des congrès des sociétés historiques et scientifiques), 2017. \\ Cet article a été validé par le comité de lecture des Éditions du CTHS dans le cadre de la publication \\ des actes du $139^{\circ}$ Congrès national des sociétés historiques et scientifiques tenu à Nîmes en 2014.
}

Une entreprise ne produit pas que des objets, de l'organisation et des procédés. Elle communique, en interne et en externe, et produit donc aussi des discours et des images qui constituent et révèlent un imaginaire. Cette dimension "software » de l'entreprise a assez peu été explorée par l'historiographie ${ }^{1}$. Certainement à tort dans la mesure où la communication est une source idéale pour accéder à la culture d'entreprise et à son système représentationnel. Cette contribution s'inscrit dans un travail au long cours de ses auteurs, attachés à l'émergence d'une histoire culturelle et patrimoniale de l'entreprise 2 .

C'est une des plus vieilles et plus importantes entreprises automobiles françaises que nous avons choisie pour illustrer notre propos: Peugeot. Grâce à la découverte d'un fonds d'archives photographiques, nous avions tenté, naguère, d'appréhender l'image comme un outil d'investigation à part entière, considérant que l'image a une vertu informative et « véritative » (P. Ricoeur), et pas seulement illustrative ${ }^{3}$.

Nous souhaitons ici nous attacher à l'analyse de la communication technique de Peugeot pour essayer de montrer ce qu'elle nous dit de la manière dont cette entreprise se représente elle-même et aborde les enjeux techniques et organisationnels de son évolution. Une évolution qui court sur presque un siècle, depuis 1912, date de la création de l'usine dans la plaine de l'Allan. Nous proposons, sur un temps long et de manière comparatiste, de repérer les méthodes et outils communicationnels qui sont mis en œuvre et d'analyser le discours (champ lexical et notionnel, thèmes et mots-clés) et ses objets. Ce faisant, nous aimerions révéler les idéologies (mythes, modèles et valeurs) qui traversent (implicitement ou explicitement) ces discours et légitiment pratiques et politiques.

1. R. Huguenin, L'univers visuel de Suchard (1945-1990). Des images de l'entreprise à l'image d'entreprise ; N. Gérome (dir.), Archives sensibles. Images et Objets du monde industriel et ouvrier; K. Tenfelfde, «Le recours à la photographie : Krupp », p. 77-94 ; M. Pironi, J. Roux (dir.), Le travail photographié ; A. Michel, Les images du travail à la chaîne dans les usines Renault de Boulogne-Billancourt (1917-1939). Une analyse des sources visuelles : cinéma, photographies, plans d'implantation; N. Pierrot, D. Woronoff (dir.), Les images de l'industrie de 1850 à nos jours ; D. Woronoff, La France industrielle. Gens des ateliers et des usines, 1890-1950; N. Pierrot, «Les images du monde industriel », p. 223-228.

2. R. Belot, P. Lamard, Image[s] de l'industrie, XIX et $X X^{e}$ siècles, p. 33-55.

3. R. Belot, P. Lamard, Peugeot à Sochaux, des hommes, une usine, un territoire. 


\section{Une première communication de nature technique destinée à l'encadrement}

\section{Informer pour former à une nouvelle culture de l'organisation du travail}

C'est d'abord un souci de formation qui a conduit l'entreprise Peugeot à prêter attention à la communication. En effet, il fut un temps où l'information était rare et la formation des hommes n'était pas pensée de manière systématique et didactique. Il s'agissait essentiellement d'un transfert pragmatique de pratiques et de savoir-faire.

La nécessité et l'utilité d'une prise en compte de la communication se sont fait sentir quand Peugeot est passé à un nouveau mode de production. À cet égard, la Première Guerre mondiale a été un moment charnière. On sait que les fabrications de guerre ont été un test grandeur nature d'une nouvelle manière de rationaliser la production. Les décideurs ont compris que l'industrie de la très grande série, qui deviendra de masse, industrie de demain, serait d'abord une affaire d'organisation. Après l'ère des bricoleurs de génie vient le temps des ingénieurs et des organisateurs. C'est le moment où se popularisent les idées tayloriennes.

La publication d'Henri Fayol en 1918 (Administration industrielle et générale) annonce l'émergence de la science de la gestion et du management et sa diffusion dans l'entreprise. La famille Peugeot épouse ce parti pris de la modernité dès 1912, lorsque Robert, dans une intuition assez géniale, achète 200 hectares sur la plaine de l'Allan à Sochaux, dans le Pays de Montbéliard pour y construire une nouvelle usine ${ }^{4}$. Peugeot est alors le deuxième constructeur français. Elle va confier à Ernest Mattern, un ingénieur de génie qui achève sa carrière comme directeur général des Fabrications et des Services techniques des Automobiles Peugeot, le soin de mettre l'entreprise sur les rails du taylorisme, de la planification et du management ${ }^{5}$. C'est lui qui va tenter de coordonner le travail des différentes usines Peugeot (essentiellement Beaulieu et Audincourt ${ }^{6}$ ).

Les premiers numéros du Bulletin des usines, la guerre à peine achevée, portent témoignage de ce mouvement qui se manifeste notamment par un engouement en faveur des méthodes américaines ${ }^{7}$. On cite longuement Charles Schwab, le grand industriel américain directeur de la construction navale des États-Unis pendant la Grande Guerre. Un symbole bien américain des «leaders sortis du rang » qui, sans être des "prodiges », ont réussi parce qu'ils ont su travailler plus que ce qui leur était demandé. Le 21 mai 1919 est publié un article de Bertrand Thompson, ancien collaborateur de Taylor, qui s'intitule L'organisation scientifique des usines. On y apprend que les principes doivent être basés sur les méthodes, et non pas l'inverse. Une formation trop théorique est considérée comme inefficace : il ne suffit pas de connaître parfaitement les lois de la thermodynamique pour construire un moteur en état de fonctionner. La France doit imiter l'Amérique qui a fait profession de pragmatisme et croit au "progrès de la science appliquée ». Une révolution culturelle est à faire en France, plaide l'Américain.

Le primat de l'organisation implique de penser à la fois la formation (d'où la volonté de créer des écoles d'apprentissage à partir de $1919^{8}$ ) et la communication, et tout particulièrement la communication technique et les nouvelles valeurs et méthodes de

4. Il s'agit au départ de fabriquer des petits utilitaires : châssis de camions, autocars et voitures de livraison. Le ministère de la Guerre voulait stimuler ce type de productions. Les voitures de tourisme, les cycles et motocycles sont produits par d'autres sites.

5. Mattern occupe ce poste éminent de 1917 à 1941, avec une coupure de la fin 1922 au début de 1928 qui correspond à son passage chez Citroën. Sur les débuts de Mattern à Peugeot, on se reportera au livre fondamental de : Y. Cohen, Organiser à l'aube du taylorisme. La pratique d'Ernest Mattern chez Peugeot, 1906-1919. 6. Et subsidiairement Levallois et Issy-les-Moulineaux.

7. Les sources non spécifiées proviennent du Centre de Documentation et d'Archives de Peugeot (Sochaux), devenu en 2010 Centre d'Archives de Terre Blanche.

8. P. Lamard, "Le paysage de la formation ouvrière chez Peugeot et Alsthom (1914-1970) ", Les Cahiers de RECITS, 2015. 
l'art de produire. La communication n'est pas seulement une manière de distribuer de l'information. Elle sert un projet plus vaste d'intensification de la "rationalisation » et de la «coordination », les deux maîtres mots de Mattern. Il s'agit d'unifier, de maîtriser, d'éduquer.

La «préface » du premier numéro proclame ce nouvel état d'esprit, après avoir rappelé que la Victoire oblige l'entreprise à intensifier « le meilleur de ses forces accrues » :

«Pour cela, le bon vouloir de tous lui sera nécessaire et, plus encore, la coordination des moyens de chacun, leur organisation rationnelle, leur orientation définie vers des buts connus, toutes conditions dont la collectivité recueillera, par une réussite immédiate et certaine, la juste prospérité qu'elle attend. " ${ }^{9}$

Derrière la dimension technique et formationnelle, il y a la volonté de créer un sentiment d'appartenance, de provoquer une unicité des pratiques pour mieux acculturer les nouvelles méthodes de travail. D'ailleurs, " des collaborateurs, d'origine très modeste, sont quelquefois pour les Directeurs des auxiliaires extrêmement précieux» déclare Ernest Mattern dans ses mémoires ${ }^{10}$. Il faut créer du lien pour que chaque partie, même la plus modeste, puisse connaître (et se reconnaître dans) le tout afin de se mobiliser en faveur du projet collectif et de partager des valeurs et une culture communes. Pour cela, il convient d'informer les gens sur la marche de l'entreprise. Une dimension d'autant plus impérative que l'entreprise se développe, que les effectifs croissent et que les tâches se parcellisent et se spécialisent :

«[...] si dans plusieurs branches de fabrication, la spécialisation demeure indispensable, si tous ne peuvent prétendre à une compétence embrassant la diversité des travaux, tous néanmoins doivent désirer connaître ce qui se fait à côté d'eux. L'effort commun n'en devient que plus efficace et ses multiples composantes, liées en un même faisceau, n'en concourent que plus étroitement au résultat cherché, partant au mieux-être de tous. C'est dans ce but que nous entreprenons la publication du Bulletin des Usines. ${ }^{11}$

\section{Peugeot, pionnier de la presse d'entreprise}

Le Bulletin des Usines Peugeot, premier bulletin de liaison interne, est créé en 1918. L'entreprise est pionnière dans ce domaine. Seul Berliet l'a devancée, en 1916, avec L'Effort. L'idée en revient à Ernest Mattern, le puissant directeur technique des usines du Doubs. Ce qu'il vise, comme le proclame le premier numéro, c'est :

« Développer les initiatives et ouvrir les esprits aux idées nouvelles. »

Avec l'ingénieur Mattern, la marque au Lion quitte le monde des ateliers familiaux qui dominent le Pays de Montbéliard pour entrer de plain-pied dans l'ère industrielle. Ce passage a provoqué une sorte de traumatisme dans les habitudes. En juillet 1922, un mouvement social est déclenché. Pour la première fois, E. Mattern incarne au sein de la société l'autorité avec laquelle il faut négocier, les Peugeot ne sont plus en première ligne. Plus fordien que jamais, Mattern, dès le retour de la paix, souhaite aller encore plus loin dans la restructuration et engager l'entreprise sur la voie du modèle unique en grande série et de la généralisation de la chaîne de montage ${ }^{12}$. Les grandes directions (technique, ressources humaines...) voient leurs prérogatives s'affirmer quant à la gestion au quotidien des usines. Mais Mattern s'intéresse d'abord à la maîtrise et à la formation d'une nouvelle maîtrise. Le Bulletin des usines ne sera distribué qu'à partir du niveau de chef d'équipe. C'est par le haut que la réforme doit s'imposer et l'impulsion se propager.

9. Centre d'Archives de Terre Blanche PSA Peugeot-Citroën, Le Bulletin des Usines Peugeot, $\mathrm{n}^{\circ} 1$, mercredi 17 juillet 1918 .

10. E. Mattern, « Principes à observer pour la Direction d'une Société », p. 2.

11. Centre d'Archives de Terre Blanche PSA Peugeot-Citroën, Le Bulletin des Usines Peugeot, $\mathrm{n}^{\circ} 1, \mathrm{mercredi}^{\circ}$ 17 juillet 1918 .

12. Y. Cohen, «Un ingénieur et sa pratique. Les techniques et la subjectivité », p. 77-210. 
Il s'agit de former les cadres de l'entreprise à une culture nouvelle: la culture d'innovation, de rationalisation et d'implication.

Ce premier journal Peugeot témoigne d'une nouvelle philosophie managériale. L'organisation scientifique des usines dépend des méthodes mais aussi des hommes et de la manière de les utiliser. Le système taylorien vise à responsabiliser des individus, grâce au " principe d'exception » qui consiste à réserver à la décision d'une autorité supérieure uniquement les seules questions qui ne peuvent être résolues par les subordonnés dans la hiérarchie. D'où le rôle du «contremaître » dans le système américain : "l'autorité du contremaître spécialisé est absolue dans la sphère clairement définie qui lui est dévolue ».

Cette idée est reprise dans le numéro $4 \mathrm{du}$ Bulletin des Usines (18 décembre 1918), à la rubrique «Demain », où est reproduit un article («L'intelligence dans la production ») paru dans La Démocratie Nouvelle:

«Mais nous avons appris à nos dépens que les plus importantes découvertes restent stériles s'il n'intervient pas une troisième catégorie de grands esprits, dits organisateurs, inventeurs, d'une autre sorte, qui possèdent le don de créer et de faire vivre de vastes entreprises parce qu'ils ont le sens aigu des réalités et savent mener les hommes. On les appelle des chefs d'industrie, mais ceux-ci ne peuvent agir à leur tour que s'ils sont secondés par des employés supérieurs, des techniciens, des contremaîtres, etc. La civilisation moderne est donc le produit d'une élite qui comporte des grades variés, où peut pénétrer tout homme accusant une supériorité de connaissances, de talent, ou d'énergie. »

La figure du contremaître est omniprésente dans la presse Peugeot. Plus, d'ailleurs, que celle de l'ingénieur. Il est présenté comme étant le cœur de la révolution taylorienne que Peugeot souhaite s'approprier. Dans le numéro $7 \mathrm{du}$ Bulletin des Usines (22 septembre 1919), on trouve la traduction d'un article de John H. Van Deventer, publié dans l'American Machinist de mars 1919, dont le titre est tout un programme : "Le credo du contremaître » :

« Je crois à la dignité de la maîtrise, à la manière de servir et à la responsabilité de la Direction. Je crois à la mission qui m'est confiée. J'ai foi en ma Maison. Sa renommée est la mienne. Je veux la garder de toute considération et, par mon exemple et mon travail, la faire grandir de jour en jour. Pour les ouvriers dont j'ai la direction, je suis la Maison et c'est d'après mes actes qu'ils la jugeront bonne ou mauvaise. Je crois à notre production. Afin de lui conserver sa qualité parfaite, je ferai tout ce qui est en mon pouvoir pour récompenser le bon travail, améliorer la qualité de travail médiocre et éliminer le mauvais travail. J'étudierai le fonctionnement de nos véhicules, leur façon de se comporter et en expliquerai à mes ouvriers le pourquoi et le comment...»

Conclusion on ne peut plus moralisatrice, bien en phase avec la mentalité américaine :

«Et ainsi, lorsque j'aurai fini mon travail, le monde dira de moi (et ma conscience aussi) : le monde est meilleur depuis que vous y avez vécu et votre industrie est plus forte depuis que vous y avez travaillé. »

L'approche de ce premier journal se veut technique et didactique. Il convient en effet de montrer la manière dont on innove ailleurs. Il faut aussi développer une culture technique et managériale. D'où des recensions d'articles, de livres ou de pratiques. Des points précis sont traités : traitement thermique des aciers, interchangeabilité des pièces, dispositifs d'usinage, innovations d'outillage, frais généraux, etc. Les allusions à l'actualité sont rarissimes. Le numéro du 18 décembre 1918 ne peut pas ne pas évoquer l'armistice. C'est l'occasion de rappeler la philosophie de l'entreprise et de ses dirigeants directement en lien avec les grandes préoccupations de la nation. Cette philosophie repose sur la revendication d'un patriotisme qui se veut intransigeant et l'acceptation de la « guerre économique » au service de l'idéal collectif qu'est la prospérité : 
«Il faut plus que jamais rester unis, plus intimement encore, car la lutte continue. C'est notre bonheur qui est en jeu. Qu'est-ce donc que la lutte économique si ce n'est la volonté et les forces des peuples dirigées vers une prospérité plus grande et plus juste? »

La solidarité nationale, que la guerre a su cristalliser, est le modèle que l'entreprise veut diffuser :

«Coupable est celui qui s'attarde à sa tâche, car tous souffriront à cause de lui ; lorsqu'une cellule ne fonctionne plus, l'organisme tout entier ne tarde à être atteint. »

L'effort de rationalisation des méthodes et des comportements va de pair avec l'exigence de mobilisation, au sens presque militaire. Enfin, l'efficacité. La guerre a provoqué des changements de méthodes dont il faut tirer parti pour la production de paix (qui est une production de "guerre économique»). Le succès dépendra de «l'intelligente organisation» des moyens de production. Outre les articles purement techniques, on trouve dans ce Bulletin des résumés d'articles ou de livres théoriques sur l'organisation de la production qui sont censés incarner ces valeurs.

La vie intérieure des usines occupe une place très modeste. Il faut un événement majeur, comme, le 22 septembre 1919, l'annonce que sera poursuivie l'application de la journée de 8 heures instaurée le $1^{\text {er }}$ juin 1919. Cette nouvelle est présentée comme un "événement social ». Mais c'est l'occasion pour réaffirmer l'exigence de «rendement » (on ne parle pas alors de productivité) :

«Le travail que nous demandons doit gagner en intensité ce qu'il perd en durée; que pendant nos 8 heures de labeur nous donnions joyeusement toutes nos forces et toute notre attention au travail. L'oisiveté est un crime. »

\section{Les rapports techniques}

Parallèlement au Bulletin, une communication très technique est déployée avec les Rapports techniques. Avec un rythme mensuel, ils s'adressent à l'élite technicienne de l'entreprise. Créés en 1918, à l'initiative de l'inévitable Ernest Mattern, ils s'arrêtent en $1970^{13}$. Leur forme est rudimentaire : des feuillets dactylographiés. Après 1945, ils sont ronéotés et pourront atteindre une centaine de pages. Dès l'origine, ils sont illustrés de tableaux et schémas soulignant leur portée didactique. C'est d'une véritable éducation à la technique dont il s'agit. Nous sommes donc dans la «boîte noire» et non dans la stratégie, qui relève du conseil d'administration et obéit à un autre usage et à un autre type de diffusion, résolument confidentielle. Mais c'est un élément important de la communication d'entreprise à l'égard d'elle-même dans une perspective mélioriste. Ces Rapports sont également un élément d'information sur le suivi de la réforme organisationnelle mise en œuvre par Mattern au début de son règne. On y apprend ce qui se passe dans les usines du groupe. Ainsi, le Rapport technique d'avril 1919 se félicite que les Services Vérification et Entretien soient définitivement constituées dans l'usine de Beaulieu, «pour le plus grand bien de la fabrication »:

«C'est ainsi que les moyeux de bicyclette se montent beaucoup plus facilement et sont mieux exécutés. Les monteurs de vélos ont aussi beaucoup moins de retouches à faire. »

La lecture des Rapports techniques est éclairante par les informations qu'ils permettent de révéler sur l'évolution des systèmes techniques, à travers notamment la recherche permanente de l'optimisation de la production et de l'amélioration tant de la qualité que de la rentabilité.

13. Selon Nicolas Hatzfeld, cet arrêt serait lié à la mise en place d'une nouvelle organisation d'Automobiles Peugeot : un système «multi-centres » qui fait perdre à Sochaux sa spécificité de lieu matriciel pour devenir un centre de production parmi d'autres. 
Ainsi, les efforts quant à une impérative et salutaire rationalisation sont précisément quantifiés pour toutes les usines du groupe Peugeot notamment au cours de l'année 1951 :

«En carrosserie, où les réalisations ont pu être poussées plus activement, sur un total de valeurs de gamme de 3 heures étudié dans le cercle de simplification, les économies réalisées à ce jour représentent 32 minutes de M. O. et 100 F de matière par voiture. »

Il arrive aussi que les retours de la clientèle soient évoqués et analysés. Des essais, des enquêtes (sur la question des horaires de doublage) et chiffrages avant application sont étudiés. En mai 1925, par exemple, dans le domaine de la peinture, nous apprenons que «les pistolets à peinture Lebaron à jet plat, modèle Chromographe $\mathrm{N}^{\circ} 9$, ont un rendement supérieur de $20 \%$ aux pistolets DeVilbiss », d'origine américaine.

«Il faut savoir trouver, au détour d'un paragraphe, une remarque de tel ou tel directeur qui va nous éclairer sur l'évolution des techniques. Si la peinture au pistolet est notée en 1919 à Beaulieu pour les cadres de vélos, Mandeure, qui fabrique des caisses dans l'ancienne usine du carrossier Gauthier, applique la peinture par «vaporisation » dès janvier 1920. En même temps la direction se plaint de l'absence de spécialistes peintres... et plus loin annonce que la mise au point des «appareils» (sans doute des pistolets) DeVilbiss devra être poussée pour suppléer au manque de peintres. Les spécialistes en question étaient donc des peintres au «pinceau » et l'application au pistolet devrait donc permettre l'embauche de personnel moins qualifié, en tout cas plus rapidement formé. " ${ }^{14}$

Il convient de préciser que, outre les grandes rubriques (production, productivité), le domaine de la main-d'œuvre et du personnel apparaît, mais il est traité en fin de rapport. $C^{\prime}$ est à la fin de leur parution seulement qu'interviennent les questions de gestion. On peut également noter que les questions extra-techniques prennent de l'ampleur à partir de 1945, en lien avec la montée en puissance du site sochalien (formations au chronométrage, à la simplification du travail, suggestions, reconfiguration des bâtiments et d'ateliers, grèves, système de transports collectifs, logements et dortoirs, travailleurs migrants, ravitaillement après-guerre...).

Les Rapports techniques permettent au haut encadrement d'avoir une idée de l'activité du "Contrôle» et de s'acculturer à la démarche qualité. Le choix des fournisseurs est l'occasion d'une évaluation permanente. Ainsi, en 1957, c'est un fournisseur textile qui est en cause. Le tissu à pavillon est de mauvaise qualité, note le Rapport d'avril 1957. Le fournisseur a été convoqué pour procéder au constat :

«Le Contrôle a été amené à faire démonter journellement un nombre assez important de pavillons non livrables et à en laisser passer de douteux par manque d'approvisionnement correct. Il est urgent de faire redresser cette situation si on veut éviter l'embouteillage en usine, ainsi que des réactions justifiées en clientèle. »

Mais les fournisseurs ne sont pas les seuls en ligne de mire. Les défauts relevés, et exposés dans les Rapports, sont aussi imputables à des erreurs, des dégradations du travail, des négligences, qui appellent des modifications d'organisation. On peut donc considérer que les Rapports techniques sont à la fois un miroir d'activité, une source de savoir et un outil d'auto-évaluation et de préconisation pour la hiérarchie.

Le caractère technique des Rapports n'empêche pas les événements (internes et externes) de s'y faufiler. Car la prise en compte des aléas et des incidents est nécessaire pour que les décideurs réussissent à mesurer l'écart qui s'installe inévitablement entre les objectifs mensuels et les résultats. Le plus souvent, ce sont les pannes (de transformateurs électriques, par exemple), les retards de livraison de pièces, de matières ou d'accessoires qui perturbent le bon déroulement du processus de fabrication.

14. Bernard Derelle, ancien ingénieur dans le domaine de la peinture. Voir : http://patrimoine-archives.psapeugeot-citroen.com/menu/index/actualite. 
Mais l'environnement extérieur a naturellement prise sur l'entreprise. Ainsi, dans le rapport de juillet 1949, on explique que, "malgré plusieurs causes de ralentissement: panne de courant de 1 heure le 15, Tour de France le 22, grève de 1 heure le 27, la réalisation du programme a suivi l'arrivée des châssis ». Grâce à ses Rapports, on pourrait reconstituer l'histoire des grèves à EDF et leur impact sur une grande entreprise comme Peugeot!

Ainsi, le 15 octobre 1957, la direction apprend que, par suite d'arrêts de travail, les usines seront privées d'énergie électrique le lendemain. Elle prend donc la décision d'arrêter les usines pour 24 heures et l'annonce au Comité d'Établissement et au personnel par téléphone et par les gardes aux portières. Le Rapport d'octobre 1957 rend compte de l'événement:

«La grève de l'EDF est totale le 16 octobre, et les usines privées complètement d'énergie. [...] Pour compenser les pertes de salaires et de production résultant du chômage, la décision est prise de faire travailler les usines le samedi 19 octobre suivant l'horaire général habituel, soit de 6 h 45 à 12 heures et de 13 h 30 à 17 h 45 . Il a été enregistré les absences suivantes au cours de cette journée parmi le personnel ouvrier : matin $17 \%$, et après-midi $26 \%$.»

La situation politique générale (Occupation, Algérie, Indochine...) et les caprices du temps (qui parfois perturbent les ramassages) ont également toute leur place dans ces Rapports, qui nous disent que la technique n'est pas séparable de l'organisation et de l'environnement.

Pour résumer, on pourrait dire, à l'instar de Nicolas Hatzfeld, que les Rapports techniques " éclairent à la fois sur les structures, les ressources et les acteurs, et les acteurs interviennent dans ce fonctionnement ${ }^{15}$. Ces Rapports sont une source documentaire majeure grâce à laquelle l'historien a pu écrire l'histoire de l'usine de Carrosserie à Sochaux.

\section{Peugeot-Revue, pour les heureux possesseurs d'une automobile Peugeot}

La communication Peugeot conçue par l'ingénieur Mattern repose sur trois piliers. Aux deux premiers piliers, voués à la technique et à l'information interne, il faut ajouter un support qui se présente de prime abord comme non-technique et qui s'adresse à l'extérieur de l'usine. En 1923, un nouveau média est lancé : Peugeot-Revue. Mais cette revue papier glacé assez luxueuse, qui s'arrêtera en 1934, est l'organe du groupe (et pas seulement du site sochalien) et s'adresse à l'extérieur et d'abord aux possesseurs d'une Peugeot. Cette revue, qui mériterait une étude spécifique, comporte des informations techniques accessibles pour l'acquéreur ou le futur acquéreur d'une Peugeot. Mais elle est centrée sur le produit et comporte des publicités abondantes agrémentées de nombreuses caricatures. Il s'agit plus ici de faire rêver. Pourtant, comme le verra, la culture austère et protestante des Peugeot ne porte pas naturellement l'entreprise vers le discours publicitaire, comme si la publicité avait un caractère dégradant et fallacieux.

On observe que les supports tournés vers le grand public sont imprégnés de culture technique qui doit garantir le sérieux de l'automobile Peugeot. Un exemple: Peugeot entend très tôt s'inscrire dans une proto-démarche "qualité », comme en témoigne un numéro de Peugeot-Revue qui lance une « Référendum Peugeot » :

«Faites-nous ci-dessous la critique (en bien ou en mal) des différents organes de la voiture et dites-nous les qualités et les défauts que vous avez constatés. »

En 1924, Peugeot-Revue consacre un long historique à la marque. Dans la première partie intitulée Une grande famille d'industriels, l'auteur explique que s'il était ministre de

15. N. Hatzfeld, Les gens d'usine. 50 ans d'histoire à Peugeot-Sochaux, p.153. Certains exemples cités ici ont été tirés de cet ouvrage. 
l'Instruction publique, il modifierait les programmes d'histoire. Au lieu de bourrer la tête des jeunes avec des batailles, des mots héroïques et des noms de maîtresses de rois, il faudrait évoquer l'importance des «questions économiques» et saluer le rôle des entreprises dans la grandeur de la France :

«La grandeur de notre pays, la prospérité de certaines de nos provinces sont dues à l'initiative, à l'intelligence et au travail opiniâtre de braves artisans qui auraient droit, plus que les favorites de nos rois, à voir leurs noms dans les manuels d'histoire. »

\section{Vers une communication de masse}

\section{Le Trait d'Union, ou comment parler à l'ensemble des acteurs de l'entreprise?}

L'entrée de Peugeot dans l'ère des méthodes américaines est une rupture qui provoque des craintes. La parcellisation des tâches comporte un risque de fragmentation de la communauté de travail et de dilution de l'espèce de patriotisme d'entreprise qui est un trait caractéristique de la culture Peugeot, qu'on a tant assimilé au «paternalisme ». Communiquer à une échelle plus large devient une nécessité. C'est en octobre 1929 que l'ensemble du personnel des usines du groupe Peugeot (autos, cycles, outillage, quincaillerie) devient destinataire d'un périodique gratuit qui prendra le nom de Trait d'Union Peugeot. Il est officiellement «l'organe mensuel des établissements Peugeot dans le département du Doubs ». La direction l'a conçu comme un facteur de cohésion tout autant qu'un outil d'information, en un temps où le développement des effectifs ne permet plus l'établissement de rapports personnels avec la haute hiérarchie et une circulation de l'information fluide :

«Les dirigeants des différentes usines estiment en effet que la collaboration qui a toujours existé entre eux et tous les membres du personnel est le premier facteur de la prospérité des usines du groupe : de cette prospérité résulte le bien-être de chacun. Mais si les contacts directs avec les dirigeants étaient fréquents et faciles quand les usines n'occupaient que peu de monde, ils sont malheureusement plus difficiles à établir aujourd'hui à cause de l'extension qu'elles ont prise. ${ }^{16}$

Cette publication correspondant au retour dans le Pays de Montbéliard d'Ernest Mattern, il est clair que le Trait d'Union participe de cette volonté de renforcer le processus d'unification et de spécialisation des sites et des usines. Il y a aussi, très certainement, le souci de ne pas laisser les syndicats se constituer en uniques médiateurs et pourvoyeurs d'informations.

Cet «heureux équilibre» est l'arme des Peugeot pour faire en sorte que les relations sociales de l'entreprise ne soient pas vécues sur un mode violent. D'où le thème, récurrent, de la valorisation des progrès à la fois techniques, économiques et sociaux réalisés par la communauté et qui sont sensés rejaillir sur chaque membre de cette communauté, quel que soit son rang. Au moment du Front Populaire, le journal justifie et vante les résultats de sa politique. Peugeot explique que la plus grande partie de ses bénéfices est réinvestie dans l'outillage des usines, ce qui permet d'accroître leur puissance de production et d'améliorer la précision et la qualité des produits. Ceci « pour le plus grand bien de tous » car la modernisation constante des machines permet de soutenir la lutte contre les concurrents mieux outillés. Elle permet aussi d'alléger la pénibilité du travail humain.

L'intérêt bien compris des travailleurs, martèle le Trait d'Union, est de ne pas dissocier leur destin de celui de l'entreprise. La créativité technique et l'investissement dans la machine sont un gage de prospérité tant économique que sociale. Ce credo Peugeot 
apparaît parfaitement dans la conclusion du numéro spécial de l'automne 1937, célébrant le vingt-cinquième anniversaire de la création de Sochaux :

«Il faut de toute nécessité que la diminution du nombre des heures de travail soit compensée non seulement par les progrès du machinisme et des méthodes de travail, mais aussi par un effort énergique du peuple des travailleurs soutenu par le sentiment que son propre intérêt ne se peut séparer de celui des entreprises. [...] Nous sommes persuadés que tous auront à cœur, au lieu de s'épuiser dans des luttes stériles, de collaborer sans arrièrepensée, en se rappelant que tous à la Maison Peugeot, du plus petit au plus grand, travaillent à la même œuvre pour la prospérité commune. »

Le journal d'entreprise est conçu comme un miroir de ceux qui y travaillent et comme le signe extérieur d'une "communauté de travail ». Celles et ceux qui font l'entreprise au quotidien, les décideurs comme les cadres et les exécutants, doivent sortir de l'anonymat. On montre des visages. Visages individuels ou en groupes. Dans le premier numéro du Trait d'Union, un grand édito énonce le credo de l'entreprise ("Produire») mais il est flanqué d'une photo où l'on voit «quelques vétérans des Automobiles Peugeot toujours solides au poste ", comme Émile Pheulpin, chef d'atelier à la Carrosserie, 41 ans de service. Parfois, on jette un projecteur sur un groupe. Comme la maîtrise des ateliers de peinture et d'assemblage de caisses. On regarde les travailleurs au travail et on visite des usines. On évoque des destins, lors des départs en retraite ou des décès.

Un miroir de l'individu pour mieux prendre conscience du collectif et montrer la direction où les efforts doivent converger. Au final, Le Trait d'Union a pour mission de recréer du collectif et de l'orgueil dans un nouveau monde du travail guetté par les risques de déshumanisation liés au travail " en miettes », de la production en série et à la massification de l'entreprise. Tous les journaux et magazines qui succéderont au Trait d'Union seront habités par le même souci et répondront au même schéma décliné en de nombreuses allégories. On compare l'usine à un vaisseau, à un orchestre, à une équipe de football, à un corps humain, à une grande famille.

\section{Une communication généraliste d'après-guerre : le Courrier des Usines}

La parution du Trait d'Union est interrompue en septembre 1942. Lui succède une petite brochure de 4 pages à caractère social, Communications, contrôlé par le Service central de coordination et son directeur, Pierre Sire. En mai 1945 paraît Contacts, en un seul exemplaire... Quelques mois après lui succède Avis, qui est inséré dans les enveloppes de paie. Il faut attendre avril 1946 pour qu'un lien régulier et sûr soit institué pour les usines Sochaux-Montbéliard. Ce sera le Courrier des usines. Un changement important intervient au cours de l'année 1955 : ce journal devient magazine, avec papier glacé. Le Courrier des usines devient Courrier des automobiles Peugeot le $1^{\mathrm{er}}$ janvier 1960.

Le Courrier des usines innove en consacrant deux pages aux arts et au tourisme. Il s'agit d'une communication assez généraliste. Ce journal évoque les questions qui touchent les salariés hors de l'espace strict du travail : le logement, les constructions pavillonnaires, les magasins Ravi, le nombre de voitures vendues au personnel, l'évolution et l'extension du site productif, les liens entre les usines. À partir de $1947^{17}$ et tout au long des années 1950, les récits de voyages aux États-Unis abondent, notamment ceux de Jean-Pierre Peugeot. Bien qu'il y ait des zooms sur certains métiers («Nous les fondeurs», par exemple), tout se passe comme si Le Courrier des usines et le Courrier des automobiles Peugeot se délestaient de l'information technique sur les Rapports techniques qui, comme on l'a vu, prennent de l'ampleur.

Des sujets très généraux sont abordés. L'automobile, le fisc et les charges sociales sont des thèmes récurrents. La situation de l'industrie française est régulièrement évoquée. Ainsi, en février 1952, le Courrier des usines commente la fermeture de Salmson, intervenant

17. Centre d'Archives de Terre Blanche PSA Peugeot-Citroën, «Voyage aux USA », Courrier des usines, $n^{\circ} 8,1^{\mathrm{er}}$ janvier 1947. 
après celle de Talbot. Une certaine presse de l'époque déplore ces faillites de l'industrie de luxe et brocarde Peugeot, Citroën et Renault qui font de la série et de la fabrication «à la va vite». Le journal de Sochaux se défend et considère que la fabrication en grande série a la «même valeur que la fabrication au compte-gouttes ». Cette valeur est fondée sur les contrôles et le credo de la qualité est régulièrement récité. D'où ce rappel à l'ordre aux travailleurs :

«Travailleurs de nos usines, si vous voulez que nos voitures continuent à être populaires, malgré les charges écrasantes auxquelles nous avons à faire face, il vous appartient d'apporter tous vos soins à votre tâche si humble soit-elle, car votre prospérité est liée à la qualité et par conséquent au succès de nos voitures. Et si nous voulons vivre, il est nécessaire que nos voitures soient meilleures que les autres. »

Bien que le modèle américain domine, le journal se plaît à vanter les prouesses de la technologie nationale. En 1959, le Courrier des usines, soucieux de contrecarrer ceux qui «dénigrent sans cesse la France», lance une série d'enquêtes sur «les grandeurs de la technique française ». On y apprend, entre bien d'autres choses, que le réseau aérien le plus étendu du monde est celui d'Air France, que le calculateur électronique le plus rapide est français, que le plus grand port pétrolier est français, que la plus longue artère gazière est française, que le plus grand barrage du continent africain est français, que ce sont les Français qui ont atteint la plus grande profondeur des eaux etc. Un ouvrier de la Fonderie écrit au Courrier des usines :

«Devant l'état de misère d'un certain nombre d'habitants de la terre et même de Français, ne croyez-vous pas qu'on était plus heureux au bon vieux temps?»

Le journal réplique à ce nostalgique par un long argumentaire sur la nécessité du «progrès scientifique ». Sa conclusion :

« Nous avons eu l'an dernier (1950) 568000 décès; si le taux de notre mortalité avait été le même qu'en 1801, nous en aurions compté 1160 000, soit 592000 de plus. Êtes-vous certain que vous, votre femme ou un de vos enfants n'auriez pas été un de ces 592000 ? »

Un gros travail de pédagogie est fourni à partir de 1957 (traité de Rome) pour que le personnel comprenne les enjeux et les risques du Marché Commun Européen et de la libération des échanges. Un sujet qui préoccupe la direction. Celle-ci a conscience que la construction automobile française va perdre la «protection efficace » que constituent les taxes et le contingentement, ce qui fait qu'elle n'affronte la concurrence étrangère que sur les marchés extérieurs. Au départ, elle a bien du mal à savoir si c'est un « souci » ou un «espoir $»^{18}$. Puis, elle comprend que l'industrie française est restée trop longtemps « comme un boxeur qui s'est trop longtemps éloigné de la compétition». À partir de 1960, le Courrier des automobiles Peugeot consacre une page entière à « L'Europe ».

Un thème plus proche des ouvriers émerge qui concerne directement les conditions de travail : la prévention. Qu'il s'agisse de la prévention des accidents à la maison ou des accidents sur le lieu de travail. En 1950, des concours de sécurité seront organisés, confiés au Comité d'hygiène et de sécurité. On publie les noms et les photos des heureux gagnants. Au départ, il s'agit de slogans et de cas très concrets. Comment s'y prendre devant un étau-limeur, par exemple. Puis, l'information s'étoffe. On publie des études plus complètes, statistiques à l'appui. Lorsque le Courrier des usines évoque en 1951 la sécurité des yeux, il explique en juillet de cette année que le nombre d'ouvriers blessés aux yeux est de 89 ; que ceux qui ont dû arrêter le travail sont 23 ; que le nombre de journées de travail perdues est de 145 ; que le coût de ces accidents se monte à 197842 francs. En 1956, ce même journal publie les chiffres nationaux de 1953 des conséquences "d'une maladie trop souvent mortelle et qu'on pourrait appeler imprudence». Les accidents du travail, en France, auraient provoqué 2000 décès, plus de 60000 blessures

18. Centre d'Archives de Terre Blanche PSA Peugeot-Citroën, «Les entreprises devant le Marché Commun », Le Courrier des usines, décembre 1958. 
graves, près d'un million de blessures plus légères ayant entraîné un arrêt de travail. Ces accidents coûtent 60 milliards par an à la Sécurité Sociale qui indemnise 23 millions et demi de journées chômées :

« C'est comme si on immobilisait pour une année entière plus de 80000 ouvriers, l'effectif approximatif de cinq entreprises comme la nôtre. »

Il reste à savoir si ce type de communication institutionnelle a un réel impact sur les personnels. Les cadres semblent plus concernés que les ouvriers. La fin des années 1960, à travers notamment mai 68 qui a révélé la demande d'une nouvelle manière de vivre les rapports sociaux, se traduit par un changement important. Parallèlement, une page générationnelle se tourne dans la haute hiérarchie. Les dirigeants issus du rang font place à des ingénieurs qui viennent d'ailleurs et sont porteurs d'une autre culture du management. À l'instar d'un François Cusey, entré aux Automobiles Peugeot en 1962, après des études de droit, futur directeur des ressources humaines chez Citroën. Il expliquera que la volonté de «restaurer réellement la négociation sociale qui était complètement à zéro » devait s'accompagner d'une "politique de communication très puissante » :

«Il faut dire qu'avant, il y avait un journal, Le Courrier des Usines, sur papier glacé, qui ne parlait pas aux gens de ce qui les intéressait. Bon, je ne dis pas que les carnets mondains, le concert de l'Harmonie, le banquet des anciens et les remises de médailles, je ne dis pas que cela ne les intéressait pas, mais on n'y parlait pas véritablement des problèmes industriels, de ce qui faisait la vie des gens dans l'entreprise. Alors, nous avons décidé de créer le journal JIP qui était un journal engagé ${ }^{19}$. »

\section{La vie des gens dans l'entreprise et les enjeux sociétaux : le « JIP»}

Le Courrier des Usines disparaît le 13 février 1970 pour laisser naître le «JIP» (Journal d'information $d u$ personnel Peugeot). Il s'agit d'un journal et non plus d'un magazine, de format quotidien, dont la confection est confiée à une société extérieure. Sa périodicité (la quinzaine) conduit certains à penser qu'il vise à se substituer à la prose syndicale comme source d'information. C'est la première tentative d'une communication de masse. D'autres journaux existent, de moindre influence, ciblant des catégories particulières (Actuel Cadres, En Direct, Peugeot-Vendeurs...).

Le JIP entend promouvoir une information très factuelle, très pratique et très locale : les primes, les salaires, les carrières, la nouvelle présentation des feuilles de paie, l'indemnité de triplage, la prime d'horaire décalé le samedi matin, les modifications statutaires, la garantie maladie, l'extension de la Carrosserie, les travailleurs marocains, les prêts à la construction destinés au personnel, le résultat de l'élection des délégués au Comité d'établissement etc. La nouvelle politique de communication se veut en phase avec une nouvelle dynamique sociale. Le premier numéro, annonciateur du nouvel état d'esprit, porte le titre suivant : "À propos de l'accord de participation », accord signé en décembre 1970. Il s'agit de défendre et d'illustrer l'excellence de la politique sociale de l'entreprise au Lion.

Néanmoins, un thème récurrent s'impose tout au long de la décennie : les suggestions. Il faut défendre et à nouveau prendre en compte «l'intelligence ouvrière » dans les ateliers, chassée plus au moins par les méthodes fordistes qui réduisent l'O.S. à un simple rôle d'exécutant. Contribuer à améliorer les techniques de production, c'est également améliorer son pouvoir d'achat dans un rapport "gagnant/gagnant " et l'ouvrier y a désormais toute sa part. Le JIP du 25 avril 1970 est entièrement consacré à cette question et régulièrement les précieuses idées mises en œuvre sont présentées avec leurs résultats pour l'entreprise et pour leurs auteurs. On apprend que 300000 suggestions ont été ainsi examinées et traitées en 27 ans à Sochaux. Cette préoccupation revient à la fréquence d'un numéro sur quatre, tel un leitmotiv qui préfigure les grands changements du

19. Témoignage de François Cusey, cité par Nicolas Hatzfeld, Ibid, p. 380. 
système productif dans la construction automobile. Le JIP devient le miroir de cette rupture depuis la prise de conscience du danger japonais jusqu'à la mise en œuvre des nouvelles méthodes de travail. Le discours dans sa forme doit toucher le plus grand nombre :

«Apprenons un nouveau sport d'autodéfense... pour éviter que Mazda ne nous mette la pile...»

C'est un vocabulaire de combat qui est employé dans les titres : "La bataille de l'auto », "Une île qui coule» en évoquant les difficultés de l'industrie automobile britannique, «Sur tous les fronts », «Un combat sans merci», « Les Japonais en Allemagne!», « La révolution automobile »... Progressivement, les maîtres mots sont déclinés au rythme des numéros: cercles de qualité, zéro défaut, automatisation, robotisation, créativité... comme une thérapie annonciatrice d'une douloureuse modernité.

Il faut enfin remarquer que le JIP développe des thèmes émergents qui étaient absents auparavant. Par exemple la lutte contre la pollution. Un article inaugure cette nouvelle sensibilité, cette nouvelle valeur: «La pollution oui, au fait ${ }^{20} \ldots$ » $C^{\prime}$ est une époque où l'opinion accuse Peugeot de polluer le site de Sochaux : des flaques d'huile dérivant sur le cours de l'Allan en aval de Montbéliard ont été trouvées le 15 janvier 1973. Cet article évoque les nouvelles techniques de dépollution: "collecte des eaux de Mécanique, traitement des huiles solubles au sud de tous les ateliers de Mécanique... » Un article de 1975 annonce la mise en place de stations de détoxication pour 1975, puis le recyclage des eaux par une réutilisation en circuit fermé des eaux pour le refroidissement des machines. La question de «l'environnement "se renforce dans les années 1980: l'eau, l'air et les déchets. Le 30 juin 1989, le JIP titre : «Une usine propre ». Et en octobre 1992 : «Conjuguer industrialisation et protection de l'environnement ».

\section{À l'aube de l'an 2000 : Planète}

C'est en octobre 1999 que le journal redevient magazine avec Planète. Cette appellation n'est pas propre à Sochaux : il y a Planète Sochaux et il y a Planète Groupe. L'entreprise entend se délier de son attachement territorial pour être en phase avec la «mondialisation ». L'identité du site historique doit se diluer, d'autant que Peugeot a absorbé une autre marque : Citroën (1976).

Planète Sochaux se présente comme un magazine de belle qualité, sur papier glacé, d'environ 16 pages. Les textes sont beaucoup moins longs que ceux des journaux qui l'ont précédé, mais plus percutants. L'image y domine. Avec des photos qui permettent d'identifier et de valoriser une équipe, un homme ou une femme, une fonction. Des portraits, avec interviews, permettent de mieux appréhender des métiers. Un dossier thématique de quatre pages permet d'approfondir une question transversale. Par exemple le handicap et l'égalité des chances.

Le directeur du site signe l'éditorial. Sa vision est panoramique. Tout ce qui concerne les loisirs a disparu dans Planète Sochaux parce que le bulletin du Comité d'établissement y pourvoit de manière très dynamique. La nouvelle communication du Centre de production n'est pas simple à imaginer dans une entreprise qui se veut mondiale et qui ne souhaite pas survaloriser le site historique de Sochaux. Comment valoriser l'image d'un site qui est à la fois lieu de mémoire et laboratoire de la modernité ? Comment concilier le fort attachement identitaire des salariés avec l'appartenance à un grand groupe où Peugeot n'est plus seul ${ }^{21}$ ?

Un journal d'entreprise n'a plus la fonction qu'il avait avant l'ère d'Internet. D'abord, il existe des journaux d'unité: Fondeur Info, Le Câble du Mécano, etc. Ensuite, la

20. Centre d'Archives de Terre Blanche PSA Peugeot-Citroën, JIP, 11 avril 1973.

21. Témoignage aux auteurs de Jean-Charles Lefebvre, directeur de la communication. 
communication passe par d'autres médiums. Outre intranet, qui touche tous les niveaux de la maîtrise, la pratique de «la ligne arrêtée » permet une communication orale et un échange au sein de chaque usine.

\section{Le discours de l'image}

\section{Le discours publicitaire : l'information avant l'émotion}

La publicité, par nature orientée hors de l'entreprise, est aussi une manière de communiquer sur la technique. C'est même une caractéristique de Peugeot, du moins jusqu'en dans les années 1950, que de refuser un discours publicitaire trop esthétisant et enjôleur. La culture Peugeot montre une suspicion tendancielle à l'encontre de la mode. Une Peugeot doit durer et savoir résister à la volatilité des engouements. Dans un encart paru dans Peugeot-Revue, en 1931, qui montre la préparation d'une campagne d'affichage, on découvre ce slogan qui laisse entrevoir une réticence à la publicité : "La publicité fait connaître, la qualité fait vendre ».

Il y a même chez Peugeot un refus affiché de l'engouement publicitaire de l'époque. «Peugeot ne fait pas de publicité. » Ce slogan, qui claque comme une provocation lancée à l'encontre de ses concurrents, a été fièrement imprimé en bonne place dans tous les grands quotidiens de Paris et de province pendant le Salon de 1924. Cette bravade est une ingénieuse prétérition mais elle révèle également une certaine culture.

Il y a deux sortes de publicités, pour la firme. L'une est employée :

«Dans les parades de foire par les saltimbanques qui montrent gratuitement ce qu'ils ont de mieux comme artistes et comme boniment pour attirer la foule et qui n'offrent plus rien aux spectateurs qui pénètrent dans la baraque. »

Peugeot n'hésite pas à affirmer que ce genre de réclame est souvent en usage dans l'industrie automobile :

«On vante outrageusement les mérites d'une voiture au moyen d'articles, de brochures, de prospectus et d'affiches, et, lorsque le client a payé, on ne s'inquiète plus de lui. Si l'automobile ne marche pas, c'est qu'il s'en est mal servi : qu'il aille à tous les diables. »

La Société Peugeot se flatte de promouvoir la méthode inverse.

« Le client l'intéresse à partir du jour où il a acheté une Peugeot; à dater de ce moment, c'est pour lui que l'on fait tous les sacrifices, c'est pour lui que l'on dépense le budget de publicité. »

Toutes ces raisons permettent de mieux comprendre pourquoi Peugeot a moins développé la dimension publicitaire au début de l'aventure automobile. Sa communication grand public passe essentiellement, et ce dès l'origine, par le sport automobile, qui permet d'affirmer la solidité et la qualité de la technologie Peugeot. Le discours publicitaire est souvent technique et peu enclin à l'esthétisme. Nous pouvons prendre à témoin la 201, qui représente une innovation majeure en matière de communication puisque, pour la première fois de son histoire, Peugeot décide de lancer une véritable compagne publicitaire pour son lancement, en 1929. Cette campagne s'inscrit dans la stratégie de l'ingénieur Mattern.

Les roues indépendantes, invention Peugeot appliquée pour la première fois à cette voiture, font l'objet de nombreuses affiches et dessins. Il y a la présentation purement technique de cette technique, qui insiste sur la sécurité et le confort que va procurer ce système à l'acheteur de la 201. Les avantages représentés par cette innovation technique sont parfois évoqués de manière non-technique. Sur certains dessins, on voit un arc-en- 
ciel qui a vocation à faire naître un sentiment de sérénité. Il arrive qu'une affiche mette en avant le fait que la 201 a été testée sur le circuit de Montlhéry. On veut donner à penser que Peugeot ne recule pas devant des dépenses supplémentaires pour assurer la robustesse et la fiabilité de ses voitures. Ceci, bien sûr, pour susciter la confiance des acheteurs. L'autre vertu de la 201, c'est l'économie. Comme elle est petite, quoique performante, il faut convaincre, en ces temps de crise, qu'elle n'obérera pas le budget familial. De nombreuses affiches illustrent ce thème. En général, on représente une borne kilométrique pour signifier qu'avec une 201, le kilomètre est « moins cher ».

Sur ce thème, le catalogue de présentation de la 201 se montre très inventif. La déclinaison se fait autour de trois idées :

- une Peugeot ne se déprécie pas ; elle est semblable à l'or,

- une Peugeot peut se renégocier à l'occasion sans problème car c'est une valeur sûre,

- une Peugeot, c'est l'alliance inédite du luxe et de l'économie.

Bien que la marque Peugeot, qui trouve dans la moyenne gamme familiale son territoire d'épanouissement, cultive la sagesse et le sérieux, elle ne résiste pas toujours au culte de la vitesse. La vitesse comme attribut de la puissance et signe de fiabilité. Mais dans la publicité Peugeot, l'esprit de sérieux et de sagesse domine. Il faudra attendre le début des années 1990, avec la 106 notamment, pour que le discours publicitaire Peugeot se détende, sorte d'un cadre cartésien et flirte avec la provocation. Le slogan décalé fait son apparition.

\section{Le discours audiovisuel interne : miroir et vitrine}

Depuis les années 1930, Peugeot utilise les documentaires filmés pour faire passer des messages à son personnel ${ }^{22}$. Mais il faut attendre le lendemain de la Seconde Guerre mondiale pour que l'effort s'intensifie et laisse des traces archivistiques que l'historien peut traiter.

Un service spécialisé se met en place en 1952 (section Cinéma), chargé de développer une communication en interne, prioritairement ${ }^{23}$. Le fonds auquel nous avons eu accès ${ }^{24}$ contient des spots publicitaires, des films des rallyes auxquels les Automobiles Peugeot ont participé, d'enregistrements de matchs de football du FC Sochaux, d'informations techniques, mais aussi et surtout de nombreux films de formation. Nous sommes dans un contexte où l'image est rare, la télévision un objet peu répandu. L'impact devait donc être considérable sur le personnel. Les projections s'effectuent dans des salles semblables à des salles de cinéma : au rez-de-chaussée du Building Ouest, au Bungalow du stade Bonal et dans les locaux EGI (Entretien Général Incendie). Le public concerné est d'abord la maîtrise puis il s'élargit, sur le même principe que la presse papier. Peugeot est une des premières entreprises en France à employer le cinéma comme vecteur de formation, d'information et d'étude. Ainsi, en 1961, 18000 personnes ont vu un film, sur un total de 504 séances, dont 243 relatives à la prévention des accidents. En 1974, année la plus productive, on dénombre 1218 projections filmiques et diffusions vidéo qui ont atteint 55292 spectateurs. Il faudrait également mentionner les enregistrements de réunions et conférences, ainsi que 87 prises de vue cinéma.

22. Le développement qui suit s'appuie en partie sur le mémoire inédit de master de S. Perret-Gentil-ditMaillard, «Communiquer en interne : la sécurité en images. Mise en perspective du fonds audiovisuel du site de Peugeot-Sochaux », sous la direction de Robert Belot et Jean-Charles Lefèbvre, Université de Technologie de Belfort-Montbéliard, 2007.

23. Centre d'Archives de Terre Blanche PSA Peugeot-Citroën, «Le service Reproduction: la section cinématographique ", Courrier des Usines, n¹13, octobre 1962 ; "La section Cinéma du service Repro », Vite et Loin, n²56, septembre-octobre 1975 ; "L'ineffable Monsieur Cinéma », JIP, n²98, 28 avril 1989. Le service sera doté de moyens matériels importants. Il est fort de quatre techniciens de haut niveau. À noter que les films liés aux événementiels sont plutôt confiés à la société Valentin Audiovisuel basée à Sochaux.

24. Nous remercions vivement Henri Dubois, Guy Rota et Christian Viotte pour leurs témoignages et leur soutien actif. 
Dans ces années-là, on attribue à l'image un véritable pouvoir de persuasion et de transformation des esprits. D'autres images, militantes, circulent sur Peugeot qu'il s'agit de neutraliser ${ }^{25}$. Nous pensons notamment aux films réalisés par le "Groupe Medvedkine » de Sochaux et Besançon ${ }^{26}$, dans le sillage de Mai 68, qui sont une machine de guerre contre les conditions de travail chez Peugeot. Le film Week-end à Sochaux, tourné en 1971 avec de jeunes ouvriers, marie des séquences documentaires avec des scènes de théâtre filmé qui visent à dénoncer les «cadences » et « l'intensification » du travail à la chaîne et stigmatiser chronométreurs, agents de méthodes, services de recrutement, de logement et de gestion de la main-d'œuvre. Dans la même veine militante, on pourrait citer le film Avec le sang des autres, réalisé par Bruno Muel en 1974. Tourné à Sochaux, ce film présente sous des peintures apocalyptiques la vie des ouvriers, attaché à montrer une atmosphère de violence, $d^{\prime}$ " oppression » et l'emprise d'une entreprise sur un territoire ${ }^{27}$.

L'analyse du corpus (500) indique que la sécurité apparaît comme le thème principal de la production audiovisuelle. Non pas la sécurité des véhicules, mais la sécurité des conditions de travail, ce qui indique un des traits de la culture Peugeot: le souci de la lutte contre les accidents de travail, la prévention des maladies professionnelles, les risques d'accidents sur le site (entrées et sorties des personnels) puis, de manière plus tardive, la préservation de l'environnement ${ }^{28}$. C'est le fameux « esprit de sécurité » que l'on trouve souvent dans le discours de la Direction du site. Pour Peugeot, c'est un élément de distinction, porteur d'identité. C'est pourquoi l'entreprise communique à l'extérieur sur ce thème. Ainsi participe-t-elle, par exemple, à des concours interentreprises ou au premier concours de sécurité créé en 1951 par l'Institut National de la Sécurité. Peugeot est alors le seul constructeur automobile à être primée ${ }^{29}$.

\section{Le primat de l'information préventionniste et sécuritaire}

Notons que ce souci pédagogique et préventionniste est très présent dans la presse de l'entreprise dès l'origine ${ }^{30}$. Il apparaît dès le début du Trait d'Union, avec une petite bande dessinée de cinq vignettes qui revient de manière récurrente avec un thème nouveau, présenté de manière drolatique: "Histoire d'un Lion qui entre dans l'industrie ». Prenons la douzième séquence, parue dans le Trait d'Union en juin 1939 (n 116). Dans la première vignette, on voit un lion anthropomorphisé, désinvolte qui siffle, les mains dans les poches. Il ne daigne pas regarder l'affiche placardée dans l'atelier qui dit: "Une minute d'inattention coûte souvent un mois d'arrêt». Deuxième vignette: la queue du lion se prend dans le mécanisme de la machine-outil, l'animal hurle. Troisième vignette : le lion court en se tenant les fesses, et il regarde enfin l'affiche qui aurait dû le mettre en garde mais qu'il a ignorée. Quatrième vignette : le lion entre à l'infirmerie pour se faire panser ; on lit: "Vous serez quitte dans 15 jours ». Cinquième vignette : le lion, bandé, est chez lui, accueilli par son fils qui lui dit: "Tiens, voilà papa qui ressemble au grandpère!» Au-dessus de lui, on voit un tableau figurant le premier lion des usines Peugeot,

25. N. Hatzfeld, G. Rot, A. Michel, « Le travail en représentation dans les films militants. Caméras et micros dans les usines automobiles, 1968-1974 », p. 118-131.

26. Structure militante associant techniciens du cinéma et ouvriers, qui exista de 1967 à 1974 et dont Chris Marker fut la figure emblématique.

27. Le synopsis est ainsi présenté : «Une descente aux enfers. La chaîne chez Peugeot. Son direct et image simple, assourdissante image. C'est là l'essentiel de l'empire Peugeot : l'exploitation à outrance du travail humain ; et en dehors, cela continue. Ville, magasins, supermarchés, bus, distractions, vacances, logement, la ville elle-même : horizon Peugeot. On parcourt le circuit, tout est ramené à la famille Peugeot. »

28. En matière de lutte contre les accidents de travail, Peugeot est en avance de 10 ans sur le texte réglementaire du 4 août 1941 qui rend obligatoire les comités de sécurité. La société crée un comité de prévention des accidents du travail dès 1930. Elle obtiendra à ce titre la médaille d'or de l'Association des Industriels de France. 29. Centre d'Archives de Terre Blanche PSA Peugeot-Citroën, Courrier des Usines, n²0, novembre 1951 ; « Peugeot et Cie remporte la coupe de Sécurité 1956 », Bulletin des Usines, n¹00, avril-mai 1956.

30. Centre d'Archives de Terre Blanche PSA Peugeot-Citroën, «Pour prévenir les accidents de travail », Le Trait d'Union, $\mathrm{n}^{\circ} 1$, octobre 1929. Citons, au hasard : "Les dix commandements de la sécurité ", Courrier des Usines, $\mathrm{n}^{\circ} 85$, janvier 1958 ; «Le circuit des suggestions sécurité », JIP, n²1, 12 juillet 1971 ; "Accidents, pourquoi reculent-ils en mécanique? », JIP, n¹52, 21 juillet 1978. 
avec la date de $1819 .$. La sécurité sur le site et dans l'atelier constitue un des grands thèmes récurrents tant de la presse de l'entreprise que de sa production audiovisuelle.

Une base de données fait apparaître la présence de 2180 films, sans discrimination de provenance (ils n'ont pas tous été produits par l'entreprise). 201 films sont dédiés exclusivement à la sécurité, dont 115 conçus par Peugeot. On observe une montée en puissance de cette préoccupation à partir des années 1980, ce qui se traduit par une augmentation des films produits sur ce sujet.

Le périmètre qui relève de la question de la sécurité est très large. Mais on peut ainsi différencier et organiser les thèmes abordés autour de trois groupes :

- sécurité routière, manutention et incendie,

- amélioration du poste et ergonomie,

- sécurité et risques dans l'atelier.

Ce qui peut surprendre, c'est l'importance de l'attention portée à la sécurité routière, à la manutention et aux incendies. 52 films sont consacrés à la sécurité routière et 23 films à la sécurité incendie, entre 1950 et 2001. Les films concernent essentiellement la circulation lors des entrées et sorties d'usine. Cette importance témoigne de l'importance du centre de production de Sochaux, l'un des plus importants en termes d'effectifs d'Europe, dans les années 1970. Deux catégories de films : l'une destinée aux personnels, à vocation de prévention, l'autre à la Direction pour lui permettre $\mathrm{d}^{\prime}$ analyser la situation in vivo et améliorer la fluidité sur le site. Un film est consacré à la sécurité sur les abords des pistes d'essais.

La sécurité incendie (prévention, évacuation, techniques d'extinction), fléau important dans cet immense et complexe centre de production, est traitée sous tous ses aspects. On trouve de nombreux films consacrés à l'évacuation des bâtiments, qui ont la double nature de films de prévention et d'analyse d'exercice d'évacuation. La question de la manutention intervient dans ce cadre. Dans les années 1960, il s'agit d'abord de la manutention manuelle : comment soulever et déplacer une charge sans se blesser. Deux films traitent ce problème. Le film Danger Hall Carrosserie (Production Peugeot, 1962) montre le trafic quotidien en Carrosserie. Un commentaire sarcastique pointe les nombreuses situations à risques que l'on peut observer en quelques heures. Sagesse en manutention (Production Peugeot, 1968) est un film muet destiné à la formation des caristes. Il met en scène les caristes en situation dangereuse : l'un fumant devant la pompe à essence, s'endormant pendant une formation sur les extincteurs (ce qui lui aurait été fort utile lorsque quelques jours après son chariot élévateur prend feu alors qu'il est incapable d'actionner l'extincteur...).

Le deuxième centre d'intérêt est constitué par l'amélioration du poste (18 films) et l'ergonomie (27 films à partir de l'année 1960), souvent réalisés sous la direction de médecins du travail. Il s'agit d'étudier des gestes à différents postes de travail, d'en définir le caractère pathologique afin de proposer des modifications. La dimension pédagogique, toujours présente, s'inscrit dans une démarche d'autoévaluation, mettant en scène les opérateurs pour leur expliquer les bons gestes, mais il s'agit aussi de familiariser la maîtrise à l'enjeu ergonomique.

Typique est le film Gestes et postures en mécanique ${ }^{31}$, produit par l'entreprise en 1994 et réalisé sous la direction du médecin du travail Monique Deschamps. Il s'agit d'analyser les difficultés et les dangers (physiques et mentaux) provoqués par une mauvaise gestion du poste de travail en mécanique. Nous sommes en présence d'un film assez critique qui entend démontrer que les améliorations en cours ne sont pas suffisantes. Le film insiste sur le manque de concertations en amont (aux études, aux méthodes générales, à la

31. Centre d'Archives de Terre Blanche PSA Peugeot-Citroën, Gestes et postures en mécanique. Centre de production de Sochaux. Cote : 1227. Type : BVU. Production : Vidéos Sochaux. 17.01.1994. Durée : 12 minutes 45 secondes. 
direction des achats) avec les usines de fabrication. Dans la même veine, un film évoque, par exemple, les troubles musculo-squelettiques que peut faire naître la fonction de garnisseur $^{32}$ ou de finisseur. Parfois, $c^{\prime}$ est une usine qui est concernée, comme la carrosserie en $1999^{33}$. Un film présente les améliorations des conditions de travail réalisées au cours de l'année dans les ateliers de peinture du site de Sochaux, dans le cadre de la méthode «DACCORD ». L'objectif était de supprimer les déplacements inutiles, les postures torsio-lombaires contraignantes, le port de charges lourdes. Un dispositif complexe (coût: 1 million de francs) a été conçu à cette fin : basculeurs, élévateurs, tables réglables en hauteur, manipulateurs. Les conditions de travail sont alors qualifiées par la voix off de « quasi parfaites ».

Une communication soignée valorise les améliorations lancées par le service Ergonomie à partir des années 2000. Des films s'adressent à l'ensemble des personnels concernant des problèmes auxquels chacun peut être exposé, à l'instar de la vidéo École $d u$ dos, école du $g_{e s t e}{ }^{34}$. Le film débute par une explication médicale, où le dos est comparé à un amortisseur de voiture mais qui ne serait pas «interchangeable». Sont analysées les différentes maladies du dos, leurs causes et leurs conséquences. Véritable mise en garde contre les mauvaises postures, le film montre différents ouvriers au travail, évoque les gestes à ne pas faire et au contraire les gestes salutaires. Il conseille enfin une gymnastique d'entretien.

La volonté d'améliorer et de sécuriser les conditions de travail fait écho à l'exigence de simplification, thème récurrent. Le Centre de production a conçu en 1952 son premier film : Simplification du travail, d'une durée que 56 minutes $^{35}$. Il s'agit d'une présentation du poste de soudure des renforts de butées d'arrêt de porte de la 203. L'ouvrier est filmé pour décrire ses gestes, de manière à souligner la perte de temps et d'énergie. Suite à ce constat, on montre les différentes améliorations. À chaque fois, un script précise l'économie réalisée sur la main-d'œuvre, les gains de rendement ainsi que la durée de rentabilité de la nouvelle installation. Ici, les préoccupations sont économiques et non pas humaines.

Le troisième pôle thématique s'organise autour de la sécurité et des risques dans l'atelier. La formation aux règles de sécurité apparaît dans 16 films, entre 1954 et 1996. Cette thématique se déploie sur plusieurs registres. Les scénarios sont structurés sur une comparaison entre la sécurité au travail et la sécurité domestique. Ainsi en est-il du film Et chez vous comment ça va ? réalisé en 1984 par l'entreprise ${ }^{36}$. On met en scène la famille Laguigne. La première séquence présente cette famille à la maison : une voix off fait les commentaires sur toutes les situations à risques de la vie de tous les jours (à la salle de bains, lorsque l'on monte sur une chaise au lieu de prendre un escabeau...). La deuxième séquence entraîne le spectateur dans l'atelier de carrosserie où les ouvriers prennent des risques comparables : par exemple, la voix «off» rappelle que l'armoire électrique doit être fermée par l'électricien comme doit l'être l'armoire avec les produits ménagers. Le commentaire est fait sur un ton assez ironique mais le film se veut assez dramatique. Il ne montre rien mais il suggère : l'ouvrier, comme l'enfant, peut être en danger de mort lorsqu'il y a non-respect des consignes de sécurité. La conclusion du film est claire : « Ne

32. Centre d'Archives de Terre Blanche PSA Peugeot-Citroën, Garnisseurs aujourd'hui. Centre de production de Sochaux. Cote : 1335. Type : BVU. Production : Automobiles Peugeot. 25.09.1995. Durée : 8 minutes.

33. Centre d'Archives de Terre Blanche PSA Peugeot-Citroën, Amélioration R99 des conditions de travail à PEI Accessoires. Cote : 2696. Type: BVU. Production: Vidéo Sochaux. Date : 16.12.1999. Durée: 4 minutes 48 secondes.

34. Centre d'Archives de Terre Blanche PSA Peugeot-Citroën, Ecole du dos, école du geste. Cote : 2714. Type : BVU. Production: Vidéo Sochaux. Date: 26/05/2000 (cassette notée 08/06/2000, mais le film date de mai). Durée : 8 minutes 50 secondes.

35. Centre d'Archives de Terre Blanche PSA Peugeot-Citroën, Simplification du travail, Centre de production de Sochaux. Cote : 1164 et 1445 ainsi que 186 en version courte. Type : UMATIC pal. Production : Vidéos Sochaux. Date : 1952, noté Télécinéma août 1992 sur la cassette. Durée : 56 minutes.

36. Centre d'Archives de Terre Blanche PSA Peugeot-Citroën, Et chez vous comment ça va ? Centre de production de Sochaux. Cote : 227. Type : DVCAM. Production : Vidéo Sochaux, 30.09.1984. Durée : 9 minutes 15 secondes. 
soyez pas des vrais Laguigne [qui misent toute leur] vie sur la chance ». Autrement dit, le routinisme est l'ennemi de l'ouvrier.

La sécurité au poste a suscité la production de 9 films spécifiques, entre 1967 et 1997, à but d'information et de prévention. Il faut y ajouter les films dédiés à l'analyse d'accidents, qui sont au nombre de 11, de 1955 à 1984. Ce sont en général de petits scénarios, accompagnés d'une voix off, qui tentent d'expliquer que l'accident ne doit rien au hasard, que ses causes doivent être analysées (négligence, indulgence) pour éviter une réédition de l'acte. Des risques spécifiques font l'objet d'une attention, comme les risques auditifs (3 films dans les années 1980), les risques électriques (7 films entre 1954 et 1998), les risques oculaires (un seul film en 1990) et, surtout, l'alcoolisme, un fléau combattu depuis toujours auquel la famille Peugeot, qui a introduit la Croix bleue en France, est particulièrement sensible ${ }^{37} .12$ films ont été diffusés entre 1950 et 1986, mais bizarrement un seul film a été produit par la firme ${ }^{38}$.

Il s'agit de Roger ou la rencontre d'un autre type $e^{39}$. Roger, membre du personnel de Peugeot Vesoul, est alcoolique. Il rencontre des membres de l'association Amitié Peugeot-Vesoul qui vont l'aider à prendre conscience de son addiction et à en guérir. À la fin du film c'est lui qui aide un autre ouvrier à sortir de l'alcoolisme. Film muet durant le premier quart $\mathrm{d}^{\prime}$ heure, sans doute pour laisser les spectateurs s'exprimer sur ce qu'ils ont vu. Il vise lui aussi à montrer la porosité des frontières entre la vie familiale et la vie professionnelle, en montrant que l'alcoolisme a des conséquences sur la vie de famille, ruine le ménage. Par ailleurs, le film montre que Roger perd sa dignité, se ridiculise en société. Au début, il a un travail qui lui plaît, dont il est fier, puis il se retrouve à effectuer des tâches dépourvues d'intérêt qui le disqualifie. Le film insiste aussi sur le rôle que doivent avoir les autres ouvriers : il y a des personnages "positifs » qui aident Roger à se sortir de sa maladie et il y a des personnages "négatifs " qui cherchent à le faire boire, à le faire replonger dans l'alcool. Le film se termine par le message suivant :

«Toute ressemblance avec des personnes existantes n'est pas le fruit du hasard. »

Le thème de la sécurité prend une telle importance qu'il suscite la production de films spécifiquement dédiés aux nouveaux arrivants. L'un de ces premiers films s'appelle: Le trèfle à quatre feuilles ${ }^{40}$. Il débute par un discours moralisateur d'un directeur qui rappelle que si l'entreprise fait tout son possible pour que les ouvriers travaillent dans de bonnes conditions de sécurité, elle n'est pas seule en charge de cette sécurité : chacun doit se sentir comptable de sa sécurité et de celle des autres. Ce film s'inscrit dans le cadre d'un cycle de formation proposé à chaque nouvel arrivant. À la fin du film intervient un formateur avec lequel un échange est engagé.

Cette nouvelle forme de communication tente de marier l'audiovisuel, le discours, et la discussion. Quelques années après, le film d'accueil s'étoffe, comme Bienvenue à PeugeotSochaux qui présente l'ensemble du site et de l'entreprise ${ }^{41}$. Le film commence par un discours de M. Garnier, alors directeur du site de Sochaux, qui présente le site, insiste sur la qualité des hommes et des femmes nécessaire à la qualité des produits. Il présente l'entreprise, ses engagements de qualité et de professionnalisme. Les règles à respecter

37. La Croix Bleue est un organisme suisse fondé en 1877 à Genève, qui a été implanté en France en 1883 à l'instigation de Lucy Peugeot.

38. Ce qui ne veut pas dire que ce n'est pas une préoccupation constante de l'entreprise. En témoignent les nombreuses affiches diffusées dans l'ensemble des ateliers dès l'origine, ou la presse. Citons : «L'alcoolisme au travail : informer, sensibiliser, aider », JIP, n³06, 2 février 1990.

39. Centre d'Archives de Terre Blanche PSA Peugeot-Citroën, Roger ou la rencontre d'un autre type. Centre de production de Sochaux. Cote : 462. Type : UMATIC pal. Production : CNDCA coproduction association AmitiéPeugeot Vesoul, juin 1986. Durée : 34 minutes 50 secondes.

40. Centre d'Archives de Terre Blanche PSA Peugeot-Citroën, Le trèfle à quatre feuilles (version longue). Centre de production de Sochaux. Cote: 674. Type: UMATIC pal. Production: Peugeot DPRS/info. Date: 31.12.1978. Durée : 20 minutes.

41. Centre d'Archives de Terre Blanche PSA Peugeot-Citroën, Bienvenue à Peugeot Sochaux. Cote: 1400. Type: BVU. Production : Automobiles Peugeot. Date : 10.10.1996. Durée : 20 minutes. 
sont ensuite présentées et expliquées, images à l'appui, en neuf thèmes : circulation ; tenue de travail et protections de sécurité; hygiène, rangement, propreté ; travail sur machines, port de charges ; électricité ; produits à risques ; incendie ; accident et malade ; confidentialité. Il est précisé dans le film qu'une formation au secourisme sera dispensée aux nouveaux arrivants.

\section{L'intérêt heuristique de l'image animée et du thème de la sécurité}

Un des éléments majeurs de caractérisation de la production audiovisuelle est la chronologie. Celle-ci permet de mieux appréhender l'évolution du discours audiovisuel mais surtout l'évolution des préoccupations de l'entreprise qu'elle entend faire partager aux personnels.

Au départ, c'est la circulation sur le site sochalien qui polarise l'attention. Au faîte de sa puissance, le Centre de production de Sochaux est un site de 264 hectares, traversé par $20 \mathrm{~km}$ de routes et $28 \mathrm{~km}$ de voies ferrées. Le problème s'estompera au début des années 1970 avec la fermeture de la Route Nationale 463 et disparaîtra totalement avec l'interdiction, au début des années 2000, de toute circulation intérieure et la construction de parkings en périphérie. La deuxième vague thématique concerne les conditions de travail (ergonomie, améliorations de poste). Au départ, l'objectif est d'abord économique. Il s'agit de faire gagner du temps à l'entreprise. À partir des années 1980, le discours est plus humaniste et prend en considération la diminution de la pénibilité du travail et des risques de pathologie. Arrive enfin la sécurité et la question des risques. Cette montée en puissance de l'ergonomie et des risques correspond à un contexte de crise qui n'est pas favorable à l'embauche et qui a pour conséquence le vieillissement de la population ouvrière et une demande d'allégement de poste.

La sécurité (qui intègre la santé, les risques et l'amélioration des postes de travail) est une manière indirecte, mais très intime, d'évoquer le système technique et les rapports de $l^{\prime}$ homme avec le mode de production. Le film, pour Peugeot, sert trois ambitions : former (les nouveaux) ; auto-évaluer (quand un film est commandé par le médecin du travail) : améliorer le système. Pour l'historien, le film a une valeur documentaire, patrimoniale et heuristique exceptionnelle. Il renseigne sur l'évolution des conditions de travail et leur perception, sur des pratiques disparues. La sécurité permet également de révéler la manière avec laquelle l'entreprise diffuse des messages sur ses valeurs, notamment l'éthique et l'humanisme.

La production filmique nous permet de saisir une évolution majeure : comment on passe de la lutte contre les accidents de travail à un combat pour l'amélioration des conditions de travail à partir des années 1970. Est symptomatique une remarque dans le film En passant par la Franche-Comté (1954), destiné à informer les concessionnaires visitant l'usine: alors qu'un visiteur s'étonne que les pistoleurs ne portent pas de masques, l'ingénieur qui fait la visite répond que les extracteurs d'air suffisent et que les ouvriers travaillent sans danger... Le film est un miroir et une vitrine, vis-à-vis des personnels. Pour l'historien, $c^{\prime}$ est une source de premier ordre par laquelle on accède à une connaissance vivante des secteurs d'activité, des équipements, mais aussi des méthodes de travail. Comme L'Emboutissage, une usine de pointe (1988). Comme la Fonderie, rarement ouverte au public. Afin de mieux la faire connaître, de nombreux films sont créés, comme L'environnement, une volonté en Fonderie (2000). Le film, pour nous, recèle un potentiel heuristique en raison de la fonction documentaire qu'il remplit sans le savoir, et au-delà de l'enjeu et des circonstances pour lesquels il a été fabriqué. D'où son intérêt patrimonial. Il permet de se souvenir de métiers, de savoir-faire, de techniques ou de gestes disparus. Ainsi, le film Garnisseurs d'aujourd'hui (1995) réalisé par le médecin du travail Élisabeth Sérusclat dans le souci d'améliorer l'ergonomie des postes. À l'époque, on adapte une housse sur une pièce en mousse, alors qu'aujourd'hui (sachant que l'opération est externalisée) on injecte de la mousse à l'intérieur d'une housse. Mais surtout, il témoigne de l'émergence et de l'évolution des préoccupations sociétales. 
Le film n'est cependant pas à regarder comme une source unique. Loin de là. Ainsi, on observe que le sujet de la santé est quasiment absent du corpus. Par exemple le tabagisme. Cela ne signifie pas un désintérêt de l'entreprise à cette question. En effet, la lutte contre le tabagisme est omniprésente dans la presse de l'entreprise, et ce dès les années 1930, à une époque où la société ne se préoccupait pour ainsi dire pas de ce fléau $^{42}$. Le film ne remplace ni le papier ni les affiches qui sont un moyen de communiquer massivement en appui de campagne de sensibilisation. La vidéo est aujourd'hui supplantée par le support informatique et des logiciels spécifiques. À Sochaux, il n'y a plus de service spécialisé. La question se pose de savoir si l'historien de demain, compte tenu de l'obsolescence accélérée des supports, aura des ressources pour étudier l'entreprise d'aujourd'hui. La fin d'un patrimoine?

Au-delà d'une histoire culturelle du monde de l'industrie, cette approche de l'entreprise communicante produisant discours et images a pour première vertu de révéler un «matériau » historique de premier plan qui autorise de nouvelles heuristiques.

Il faudrait encourager les chercheurs à considérer les discours produits par l'entreprise comme des objets heuristiques dignes d'intérêt et non pas comme de simples adjuvants anecdotiques ou illustratifs. Prêter attention à des sources trop peu prisées, comme les archives audiovisuelles internes, constitue une promesse de renouvellement méthodologique et historiographique. La condition, c'est d'accepter de ne pas se contenter de l'angle d'approche traditionnel, se réduisant à un intérêt pour une communication de type publicitaire, commercial ou commémoratif, pour entrer dans l'univers plus intimiste et moins monstratif de l'entreprise. Il s'agit donc de s'attacher moins à l'image que l'entreprise donne d'elle-même à l'extérieur qu'au discours qu'elle tient sur elle et qu'elle adresse à son personnel.

La prise en compte de ses différents modes de communication interne (presse, affiches, films, documentaires, iconographie, photographie...) révèle et éclaire des enjeux qui touchent au « hardware » de l'acte productif et innovatif. Nous découvrons un miroir où se reflètent des préoccupations d'ordre technique, organisationnel, social, qui ellesmêmes nous permettent d'en savoir un peu plus sur la manière dont on prépare, accompagne et perçoit les mutations du système productif et des formes de gouvernance.

La société Peugeot fait figure de pionnière à de nombreux égards : précocité d'une presse interne jamais négligée par la direction, importance donnée à une administration de la communication (qui a compté plus de 200 personnes sur le site de Sochaux à la fin des Trente Glorieuses), emploi du cinéma comme vecteur d'information, de formation et d'évaluation... Quel que soit le support, apparaît une communication maîtrisée, forte, démonstrative qui reflète une intelligence perceptive face à un contexte socioéconomique et géopolitique en mutation permanente. Pour la direction, il s'agit autant que faire se peut d'anticiper puis d'élaborer une stratégie en fonction des évolutions d'un environnement de proximité ou « macro ». En effet, l'entreprise n'est jamais hors sol mais en résonance permanente avec des événements locaux comme mondiaux.

Toutefois, un grand sujet s'érige en fil rouge dans cette aventure automobile de plus d'un siècle. L'exigence de la rationalité productive parcourt toute la période sous des formes techniques différentes dont il faut faciliter l'appropriation. Il convient de sensibiliser, de diffuser principes et méthodes puis de mettre en œuvre et contrôler. La direction cherche constamment à accompagner le changement technique en misant sur les vertus d'une information préventive, formative et didactique. C'est pourquoi la communication interne y est appréhendée comme à la fois un instrument de cohésion identitaire et un outil d'éducation au changement. Et c'est en cela qu'elle intéresse l'historien : il pourra y

42. Centre d'Archives de Terre Blanche PSA Peugeot-Citroën, "Quelques conseils aux fumeurs », Courrier des Usines, $\mathrm{n}^{\circ} 40$, octobre 1953 . 
trouver les éléments qui lui permettront d'établir, dans une chronologie fine, la généalogie des grands enjeux auxquels l'entreprise est confrontée, marqueurs des époques: l'organisation fordienne, la prévention, les suggestions, la qualité... Aujourd'hui l'environnement, la santé, les risques. La prise en compte de la communication offre au regard une double focale qui permet d'entrer au cœur d'une histoire des techniques, dans ses aspects aussi bien internalistes que systémiques, mêlant l'humain, la technique, la stratégie, retraçant les succès industriels comme les échecs.

Le cas Peugeot est exemplaire pour pouvoir mesurer l'importance croissante de l'exigence communicationnelle et $\mathrm{du}$ facteur humain : réservée initialement aux ingénieurs et aux contremaîtres, elle s'étend à l'ensemble des acteurs au fil de la montée en puissance de l'entreprise sochalienne. Son périmètre thématique s'élargit dans le même temps, passant de la technique pure à la dimension sociale, culturelle et géopolitique. Après la Seconde Guerre mondiale, la communication s'inscrit dans un projet global de formation, de mobilisation, d'évaluation et d'organisation. Elle est conçue comme devant servir une pédagogie du destin commun, de l'atelier et du foyer jusqu'aux grands sujets de société extra-muros. C'est peut-être par ce biais que nous pouvons comprendre qu'une entreprise comme Peugeot a produit une culture où se rencontrent des réalités à la fois matérielles et immatérielles, une culture qui fait époque et patrimoine.

\footnotetext{
Résumé

Pionnière et exemplaire dans le domaine de la presse d'entreprise et de la communication audiovisuelle au $\mathrm{XX}^{\mathrm{e}}$ siècle, Peugeot est une est très bonne entrée pour pouvoir mesurer l'importance croissante de l'exigence communicationnelle interne et de la prise en compte du facteur humain dans les organisations fordiennes. Réservée initialement aux ingénieurs et aux contremaîtres, la presse technique s'étend à l'ensemble des acteurs au fil de la montée en puissance de l'entreprise sochalienne. Son périmètre thématique s'élargit dans le même temps, passant de la technique pure à la dimension sociétale, culturelle et géopolitique. Après la Seconde Guerre mondiale, la communication s'inscrit dans un projet global de formation, de mobilisation, d'évaluation et d'organisation. Elle est conçue comme devant servir une pédagogie du destin commun, de l'atelier et du foyer jusqu'aux grands sujets de société extra-muros. Ses supports se complexifient, intégrant à partir des années 1960 les techniques audio-visuelles qui permettent le développement d'une démarche résolument didactique et l'appropriation de la démarche qualité. C'est peut-être par ce biais que nous pouvons comprendre qu'une entreprise comme Peugeot a produit une culture où se rencontrent des réalités à la fois matérielles et immatérielles. Et c'est pourquoi l'historien ne peut plus ignorer les vertus heuristiques de la communication technique et interne de l'entreprise, à la fois miroir et fenêtre par lesquels il peut atteindre l'ontologie de l'acte productif dans ses dimensions organisationnelles et représentationnelles.
} 


\section{Bibliographie}

Belot Robert, Pierre LAMARD, Peugeot à Sochaux, des hommes, une usine, un territoire, Lavauzelle, Panazol, 2007.

Belot Robert, Pierre LAMARD, Image[s] de l'industrie, XIX et XX siècles, Paris, éd. ETAI, 2011.

COHEN Yves, Organiser à l'aube du taylorisme. La pratique d'Ernest Mattern chez Peugeot, 1906-1919, Presses universitaires franc-comtoises, 2001.

COHEN Yves, «Un ingénieur et sa pratique. Les techniques et la subjectivité », Documents pour l'histoire des techniques, nouvelle série $\mathrm{n}^{\circ} 15,1^{\mathrm{er}}$ semestre 2008, p. 77-210.

GÉROME Noëlle (dir.), Archives sensibles. Images et Objets du monde industriel et ouvrier, Cachan, Éditions de l'ENS Cachan, 1995.

HATZFELD Nicolas, Les gens d'usine. 50 ans d'histoire à Peugeot-Sochaux, 2002, Les Éditions de l'Atelier.

HATZFELD Nicolas, GWENAËLLE Rot, ALAIN Michel, « Le travail en représentation dans les films militants. Caméras et micros dans les usines automobiles, 1968-1974 ", Histoire et Sociétés, $\mathrm{n}^{\circ}$ 9, 2004, p. 118-131.

HuGUENIN Régis, L'univers visuel de Suchard (1945-1990). Des images de l'entreprise à l'image d'entreprise, Neuchâtel, Halphil, 2014.

LAMARD Pierre, «Le paysage de la formation ouvrière chez Peugeot et Alsthom (19141970) », dans Cultures et formations techniques des ouvriers et des techniciens (XVIII ${ }^{e}$ XX ${ }^{e}$ siècles), Cahiers de RECITS, $\mathrm{n}^{\circ}$ 10, Pôle éditorial UTBM, 2014.

MATTERN Ernest, "Principes à observer pour la direction d'une société», Document dactylographié, décembre 1941, p. 2, dans Documents pour l'histoire des techniques, nouvelle série $\mathrm{n}^{\circ} 15,1^{\mathrm{er}}$ semestre 2008.

MiCHEL Alain, «Les images du travail à la chaîne dans les usines Renault de BoulogneBillancourt (1917-1939). Une analyse des sources visuelles : cinéma, photographies, plans d'implantation », 2001, thèse EHESS, 2001.

PERRET-Gentil-dit-Maillard Stéphanie, "Communiquer en interne: la sécurité en images. Mise en perspective du fonds audiovisuel du site de Peugeot-Sochaux », Master HESIE, Robert BELOT et Jean-Charles LEFEBVRE (dir.), Université de Technologie de Belfort-Montbéliard, 2007.

PIERROT Nicolas, Denis WorONOFF (dir.), Les images de l'industrie de 1850 à nos jours, Paris, Comité pour l'histoire économique et financière de la France, 2002.

PIERROT Nicolas, «Les images du monde industriel », dans Gracia DOREL-FerRÉ (dir.), «Le patrimoine industriel, II », Historiens et Géographes, ${ }^{\circ} 401$, janvier 2008, p. 223-228.

PIRONI Michel, Jacques Roux (dir.), Le travail photographié, CNRS éditions, Publications de l’Université de Saint-Étienne, 1996.

TENFELFDE Klaus, «Le recours à la photographie : Krupp », Entreprises et Histoire, $\mathrm{n}^{\circ} 11$, mars 1996, p. 77-94.

WORONOFF Denis, La France industrielle. Gens des ateliers et des usines, 1890-1950, Paris, Éditions du Chêne-Hachette Livre, 2004. 


\title{
Progrès et ruptures de la communication dans les sciences exactes
}

\author{
Jean BRINI \\ Psychanalyste \\ Extrait de : Xavier LAFON, Les langues savantes, Paris, \\ Édition électronique du CTHS (Actes des congrès des sociétés historiques et scientifiques), 2017. \\ Cet article a été validé par le comité de lecture des Éditions du CTHS dans le cadre de la publication \\ des actes du $139^{\mathrm{e}}$ Congrès national des sociétés historiques et scientifiques tenu à Nîmes en 2014.
}

Nous partons de ce qui peut à première vue apparaître comme un paradoxe: notre science moderne, celle dont on peut dater l'apparition à la Renaissance avec - entre autres - Copernic et Galilée, celle qui a opéré en quelques siècles un bouleversement et une accélération de notre civilisation qui - me semble-t-il - est sans précédent dans l'histoire, et pour laquelle nous sommes incapables d'apercevoir ne serait-ce qu'un horizon qui lui ferait limite, cette science est fondée sur un postulat qui s'est avéré redoutablement efficace et qui peut s'énoncer ainsi :

«Le grand livre de la nature est écrit en langage mathématique. »

C'est en lien avec ce postulat que cette science s'est développée, en s'appuyant essentiellement sur un dispositif d'écriture en termes mathématiques des données initialement fournies par nos sens. La surprise est que ce dispositif d'écriture s'avère non seulement capable de rendre compte de nos observations - de les comprendre, en somme -, mais qu'il possède également une capacité prédictive qui nous permet de partir à la recherche de nouvelles observations, jamais effectuées jusqu'alors. La découverte de la planète Neptune est un exemple illustre de ce fait.

Les mathématiques constituent un langage à part où les règles de la dialectique sont définies de manière rigoureuse et univoque. C'est du moins le cas depuis les efforts considérables qui ont été déployés à la fin $\mathrm{du} \mathrm{XIX}^{\mathrm{e}}$ siècle pour en chasser autant qu'il était possible toute trace d'équivoque et tout recours à l'intuition. On pourrait dès lors s'attendre à ce que la transmission et le développement des connaissances scientifiques s'effectuent de manière pacifiée, la mise en œuvre de la logique mathématique pure ne laissant place dans la disputatio qu'à des arguments rationnels. Or l'expérience nous montre qu'il n'en est rien.

Si dans la plupart des cas notre science se transmet de manière indiscutable du fait de son écriture mathématique, son développement en revanche donne lieu à des controverses et à des soubresauts qui dépassent de loin le simple échange d'arguments et qui engagent les protagonistes au niveau de leur position de sujet.

Le paradoxe est donc qu'une science adossée aux mathématiques et à la logique, développant un langage dont la visée est de bannir toute équivoque dans ses concepts pour tenter d'atteindre à une objectivité idéalement absolue, se trouve si souvent entravée dans son développement par ce qu'on pourrait appeler une résurgence du sujet.

1. En 1960, le physicien Eugen Wigner publie un article au titre provocateur « La déraisonnable efficacité des mathématiques dans les sciences naturelles », (cf. E.P. Wigner " The unreasonable effectiveness of mathematics in the natural sciences ", Communications on Pure and Applied Mathematics, XIII, 1960, 1-14). 
Max Planck énonçait cela à propos de la mécanique quantique d'une manière singulièrement désabusée :

« Une vérité nouvelle en science n'arrive jamais à triompher en convainquant les adversaires et en les amenant à voir la lumière, mais plutôt parce que finalement ces adversaires meurent et qu'une nouvelle génération grandit, à qui cette vérité est familière. »²

Notre visée dans ce bref exposé sera d'abord d'illustrer ce qui est avancé ici à l'aide de deux exemples qui nous ont paru intéressants: celui de Desargues, inventeur de la géométrie projective, et celui de Cantor, créateur de la théorie des nombres transfinis. Nous tenterons ensuite de montrer que les concepts développés par la psychanalyse notamment depuis Lacan - nous donnent des indications précieuses nous permettant de lever cet apparent paradoxe.

\section{Girard Desargues le Lyonnais $(1591-1661)^{3}$}

Sieur Girard Desargues le Lyonnais, (il signait SGDL) contemporain et ami de Descartes (1596-1650), est l'inventeur de la géométrie projective. Il est celui qui le premier a su tirer les conséquences mathématiques des techniques picturales développées au $\mathrm{XV}^{\mathrm{e}}$ siècle sous l'appellation globale de perspective.

Ces techniques, il n'est pas inutile de le rappeler ici, avaient d'abord pour objet - dans la représentation, dans le tableau - de capter le regard du sujet en soulignant le caractère surnaturel des scènes représentées. Daniel Arasse a mis en valeur le lien intime qui existait entre la perspective et les représentations de l'Annonciation, scène surnaturelle s'il en fut ${ }^{4}$.

Sans revenir sur le dispositif de la perspective, dont la géométrie nous paraît souvent trompeusement simple, rappelons seulement qu'il permet de projeter rigoureusement chaque point du monde en un point précis du tableau. Apparaît alors la nécessité de prendre en compte des points à l'infini dans le monde puisqu'ils ont une image à distance finie dans le tableau: ce que nous appelons couramment l'horizon, ou plus généralement les points de fuite.

C'est Desargues $^{5}$ qui tira de cette obligation technique une géométrie nouvelle, où l'axiome des parallèles d'Euclide est remplacé par l'axiome plus simple : deux droites coplanaires se coupent en un point et un seul... toujours, même lorsqu'elles sont parallèles.

Desargues décrivait lui-même son activité de chercheur comme des contemplations capricieuses au cours desquelles «sa raison le menait là où l'entendement se perd ». Pour donner un exemple, nous pouvons citer le Brouillon project d'une atteinte aux événements de rencontre d'un cône avec un plan, traité dans lequel il renouvelle l'étude des coniques, et qui a largement inspiré le traité sur les coniques de Pascal. Dans cet ouvrage, il énumère la liste des formes que peut prendre l'intersection d'un plan avec un cône, et envisage le cas où le cône a son sommet à l'infini (il s'agit alors d'un cylindre) ou le plan est parallèle à l'axe de cône à distance suffisante de l'axe.

La courbe d'intersection des deux surfaces est alors tout entière rejetée à l'infini, Desargues en dit que «nous ne pouvons savoir ce qu'elle est». Les conceptions de Desargues rencontrèrent un accueil d'abord relativement froid de la part de ses contemporains. Il était en constante correspondance avec le groupe d'érudits qui gravitait

2. M. Planck, Autobiographie Scientifique, p. 84-85.

3. On trouvera un grand nombre d'information concernant Girard Desargues dans l'ouvrage de M. Chaboud, Girard Desargues bourgeois de Lyon, mathématicien, architecte.

4. D. Arasse, L'annonication italienne ; une affaire de perspectvie, Hazan, 2003.

5. J. Dhombres et J. Sakarovitch, Desargues en son temps, Paris, Albert Blanchard, 1994 et Taton René, L'œuvre mathématique de Girard Desargues, Paris, Vrin, 1988. 
autour du père Mersenne et reçut un certain nombre de signes d'estime notamment de la part des plus versés dans la géométrie comme Descartes ou Fermat. Néanmoins, il eut très vite des ennuis.

Dès 1640, Beaugrand édita un pamphlet contre son travail sur les coniques, en critiquant sa terminologie et en avançant que tout ce qu'il proposait était déjà chez Appolonius. En 1642 eut lieu à Paris une controverse par voie d'affiches, déclenchée par Desargues luimême, qui accusait Jean Dubreuil de l'avoir plagié, et où lui-même était accusé d'avoir commis «des erreurs incroyables, des fautes et faussetés énormes, etc. ». L'éditeur de Dubreuil publie en 1642 un recueil de pamphlets contre Desargues. En 1644, Jacques Curabelle parle là encore par voie d'affiches de la «Faiblesse pitoyable du sieur Desargues ». Girard Desargues répondit point par point à ces accusations dans un Récit au vray de ce qui a ésté la cause de faire cet escrit, mais fut finalement fortement affecté par ces polémiques, et abandonna ses activités mathématiques peu après.

Il n'est pas toujours aisé de comprendre exactement ce qui était reproché à Desargues. Parfois il s'agit de sa terminologie, parfois de sa méthode, parfois de sa prétention à avoir dégagé une "manière universelle». Son œuvre est rapidement tombée dans l'oubli, au point que certaines de ses publications, comme le Traité des ténèbres, ont été définitivement perdues et ne sont connues que par les allusions qu'y font d'autres auteurs. La géométrie projective ne revint sur le devant de la scène que lorsqu'elle s'avéra être un outil indispensable au développement de la géométrie descriptive inventée par Monge (1746-1818). La réhabilitation complète de Desargues ne fut accomplie qu'en 1864, avec l'édition de ses œuvres complètes conservées.

\section{Georg Cantor (1845 - 1918)}

Poursuivons avec l'exemple plus récent de Georg Cantor ${ }^{6}$. Cantor est essentiellement célèbre pour avoir ouvert aux mathématiques un champ qui jusqu'alors lui était resté impénétrable : celui de l'infini dit actuel. Il est connu comme le fondateur de la théorie des ensembles, qui permit au groupe Bourbaki de présenter l'ensemble des mathématiques comme un édifice unique bâti sur l'arithmétique des nombres entiers.

Parmi les résultats marquants de son œuvre, nous souhaitons en relever deux. Le premier est la création d'une arithmétique des nombres infinis (les alephs) permettant de mettre en évidence qu'il existe au moins deux types d'infini. Le premier, le dénombrable, concerne tous les ensembles infinis dont les éléments peuvent être comptés et qui sont pour cette raison équinumériques ${ }^{7}$ à l'ensemble des entiers naturels. L'ensemble des nombres pairs, des carrés, des nombres premiers, des fractions, sont de cette nature. Le second, le continu, concerne les ensembles équinumériques à l'ensemble des points d'une droite ou à l'ensemble des nombres réels.

Le second est lié au premier et concerne la notion de dimension. Cantor réussit en effet à montrer que l'ensemble des points d'une droite était équinumérique à l'ensemble des points d'un plan. De cette démonstration, il écrira son collègue et ami Dedekind :

«Tant que vous ne m'aurez pas approuvé, je ne puis que dire : Je le vois, mais je ne le crois pas. ${ }^{8}$

La création de cette écriture coûta beaucoup à Cantor, qui était profondément croyant et

6. Pour des informations sur Cantor, on consultera avec profit : J.-P. Belna, Cantor. V. Hasenbalg, Le drame subjectif de Cantor : texte disponible à l'adresse suivante : https:/ / www.mathinees-lacaniennes.net/fr/

7. Deux ensembles sont dits équinumériques lorsque leurs éléments peuvent être mis en correspondance biunivoque. On considère l'existence d'une telle correspondance comme équivalente à l'expression « ont le même nombre d'éléments ».

8. G. Cantor, correspondances, Cantor-Dedekind, Philosophie des mathématiques, trad. J. Cavaillés, Paris, Hermann, 1962, p. 179-250. 
savait parfaitement que ses travaux allaient à l'encontre de la doctrine thomiste, cette dernière avançait que l'infini actuel était réservé à Dieu, et qu'à l'homme était réservé le domaine du fini. Il passa beaucoup de temps à développer des arguments tendant à montrer que son audace était légitime aux yeux de Dieu.

Cantor rencontra lui aussi de nombreuses critiques. Ainsi, Henri Poincaré disait de la théorie des ensembles transfinis qu'elle était une maladie dont les mathématiques devaient tôt ou tard être guéries. L'un de ses maîtres, Kronecker, devint l'un de ses plus farouches opposants, déclarant «Dieu a créé les nombres entiers » et traitant Cantor de « charlatan scientifique », de « renégat » et de «corrupteur de la jeunesse ». On conçoit que ces accusations aient pu profondément affecter Cantor qui, de santé mentale fragile, fit plusieurs séjours en hôpital psychiatrique. Il finit, comme Desargues par abandonner ses activités scientifiques.

\section{Psychanalyse}

Le point sur lequel nous souhaiterions insister est le suivant. Nos deux héros, Desargues comme Cantor, témoignent dans leur œuvre d'avoir rencontré un impossible : impossible, pour Desargues d'imaginer ce que pourrait être une conique à l'infini ; impossible pour Cantor d'accepter qu'il y ait autant de points dans un plan que sur une droite.

Dans les deux cas, il s'agit d'atteindre un point où, comme le dit Desargues :

« La raison nous mène là où l'entendement se perd. »

Ce qui résulte de cette rencontre est l'ouverture d'un nouveau champ, encore inexploré de la science, et cette ouverture se trouve vivement contestée par les contemporains de la découverte.

Dans les deux cas, il ne s'agit pas d'une contestation fondée sur l'incompétence de ceux qui l'expriment. Au contraire, aussi bien les contestateurs de Desargues que ceux de Cantor sont des proches, des pairs, qui ont une connaissance approfondie du domaine étudié. Comment expliquer dès lors la violence de leurs attaques ? Comment expliquer le refus de prendre en considération les nouveautés apportées par nos deux auteurs... en somme, pourquoi tant de haine?

C'est là que nous voudrions tenter d'illustrer le fait que la psychanalyse est susceptible de jeter quelque lumière sur ce qui sans elle reste une question insoluble. Pour cela, il est nécessaire de faire quelques pas en arrière afin de nous donner une vue un peu élargie de la question.

Nous pouvons partir d'une distinction proposée par Freud dès la découverte de la psychanalyse : dans la Traumdeutung ${ }^{9}$ (la science des rêves) Freud souligne la nécessité de postuler l'existence d'une réalité psychique, distincte de ce que nous sommes convenus d'appeler la réalité tout court. Le rêve se déroule sur " une autre scène ", où d'autres lois régissent les enchaînements de représentations qui surgissent dans notre psychisme. Nous ne pouvons ici développer le détail de ce que sont ces lois. Soulignons simplement ce qui en résulte : il est inévitable de postuler l'existence d'un psychisme inconscient dont les manifestations révèlent l'existence de ce que Lacan nous a appris à désigner du terme de sujet, à distinguer de la personne.

Nous ne pouvons que noter combien telle conception est d'une certaine façon diamétralement opposée à la visée scientifique. Celle-ci postule en effet d'abord l'existence d'une réalité qualifiée d'extérieure, indépendante, qui s'avère descriptible à

9. S. Freud, La science des rêves, p. 517. 
l'aide d'un langage univoque, où chaque chose a son nom et chaque nom a sa chose.

Lacan reprendra cette distinction en soulignant qu'elle est l'effet de notre rencontre avec le langage ${ }^{10}$, et proposera de faire reposer l'ensemble des effets de cette rencontre sur ce qu'il appelle sa «robuste trilogie »: les trois registres du Réel, du Symbolique et de l'Imaginaire.

Une illustration de ces trois registres est facile à repérer dans la démarche de Desargues : ses « contemplations capricieuses », entendons le jeu des représentations par lesquelles il réfléchit aux diverses dispositions relatives d'un cône et d'un plan qui relèvent de l'Imaginaire. La mise en mots, en phrases, en énoncés, voire en théorème de ce qu'il contemple ainsi relève du registre Symbolique. C'est le développement de la combinatoire de ces énoncés organisés logiquement qui le conduit à concevoir (par exemple) l'intersection d'un cylindre avec un plan qui lui est parallèle et extérieur, et à constater qu'il lui est impossible d'associer la moindre représentation à ce que son système symbolique lui a cependant permis de concevoir. C'est la rencontre avec l'impossible qui caractérise, qui met en évidence ce que Lacan désigne du terme de Réel. Il est clair qu'on pourrait faire le même repérage pour la démarche de Cantor : il fait état de la même inquiétude lorsqu'il dit : «je le vois mais je ne le crois pas ».

Ces deux chercheurs ont, chacun pour leur compte, rencontré ce qui était pour eux à la fois présent, représenté dans le système d'écriture qu'ils mettaient en place, et cependant non représentable dans ce qu'ils étaient en mesure d'imaginer.

On peut au passage repérer ce que fait remarquer Lacan: lorsque se conjoignent symbolique et imaginaire, nous avons affaire à quelque chose qui fait sens pour nous. Lorsque l'un des deux registres manque à l'appel, nous avons affaire soit à l'Imaginaire seul: des images pour lesquelles nous n'avons pas de mots, ce qui peut occasionner une impression d'inquiétante étrangeté déjà notée par Freud ; soit au Symbolique seul, c'està-dire à une chaîne de simples lettres qui suivent leur chemin sans plus de représentation associée, c'est-à-dire à quelque chose qui n'est plus différenciable d'un délire.

Lacan n'a cessé tout au long de son enseignement de remettre sur le métier deux questions, qui pour lui étaient liées: la psychanalyse est-elle une science ? À cette question, il a répondu sans ambiguïté «non». Que serait une science qui tiendrait compte de la psychanalyse? À cette question, il n'a pas répondu, et il nous semble qu'elle est d'autant plus d'actualité que la science contemporaine nous montre son impuissance à trouver en elle-même ce qui pourrait lui faire limite ${ }^{11}$.

Pour en revenir à notre question de départ, il nous semble possible d'avancer une explication simple des difficultés rencontrées par nos deux héros. Si la science constituée est essentiellement comme un système d'écriture de type mathématique censé rendre compte des phénomènes, elle peut se transmettre sans problème, sans nulle nécessité d'aucun recours à un sujet au sens où nous l'avons évoqué ci-dessus, en ceci qu'elle se constitue en un système de signes. Rappelons la définition canonique que donne Lacan d'un signe : « quelque chose pour quelqu'un ».

En revanche, la création scientifique nécessite, et nos deux héros l'illustrent sans ambiguïté, un engagement d'un sujet dans son désir, dès lors qu'il se risque à écrire - ou d'abord à dire (Desargues dit « contempler ", Cantor dit « voir ») - quelque chose qui n'a jamais été écrit ou dit auparavant. Or cet acte de pure énonciation nécessite plus qu'un simple système de signes. Il nécessite la mise en jeu du signifiant, ce que Lacan définit comme : «ce qui représente un sujet pour un autre signifiant ».

10. Lacan J., Écrits, p. 237.

11. Le discours scientifique nous donne de plus en plus le spectacle d'une prolifération d'écritures Réelles, inscrites dans des articles, mais aussi dans le silicium et bientôt dans le génome. Cette prolifération a montré maintes fois au cours de ces dernières décennies qu'elle ne sait pas ce qui la pilote, d'où l'urgence de la question. 
La présence du sujet dans l'histoire de nos deux héros est patente dans le fait que leur œuvre a été manifestement adressée : adressée à la communauté scientifique de l'époque, adressée à des proches qui ont tenu le rôle de confident, voire de thérapeute. Cette prise de risque à laquelle ils ont consenti explique à notre sens les réactions négatives suscitées chez certains de leurs contemporains. Une étude plus détaillée des objections qui leur ont été présentées permettrait sans nul doute de montrer que leurs contradicteurs (Beaugrand, Curabelle, Kronecker) ont été eux aussi heurtés, non dans leur rationalité, mais dans leur subjectivité la plus intime. L'explication que nous avançons repose donc sur le fait simple que si la rationalité - fondée sur un système de signes univoques admis de tous - est une, la subjectivité au sens psychanalytique du terme - fondée sur un langage fait de signifiants nécessairement équivoques - est aussi nécessairement multiple.

\section{Résumé}

Le langage mathématique possède la particularité de pouvoir se traduire d'une langue à l'autre sans distorsion et sans équivoque. La constitution de notre corpus de connaissances consiste, depuis l'avènement de la science moderne, en une accumulation d'écritures dont nous attendons qu'elles rendent compte du réel d'une façon absolument consensuelle.

Or il s'avère que ce n'est pas le cas. Au contraire, les plus vives controverses accompagnent plus souvent les franchissements révolutionnaires dans les sciences dites exactes. Desargues, dont la géométrie projective fut dénigrée publiquement, Cantor, critiqué sans ménagement par Kronecker, sont des exemples célèbres de ce paradoxe : les sciences exactes n'évoluent pas selon les termes d'un débat rationnel, mais plutôt au rythme de controverses où la raison ne tient qu'une part marginale. Nous tentons de montrer qu'il est possible de rendre compte de cette contradiction à l'aide de la psychanalyse, notamment des outils que nous a laissés Lacan.

\section{Bibliographie}

BELNA Jean-Pierre, Cantor, Paris, Les Belles Lettres, 2000.

DHOMBreS Jean et SAKAROVITCH Joël, Desargues en son temps, Paris, Albert Blanchard, 1994.

FREUD Sigmund, La science des rêves, trad. Meyerson, Paris, Félix Alcan, 1926.

HASENBALG Virginia, Le drame subjectif de Cantor https://www.mathineeslacaniennes.net/fr/21-articles/articles / 95-pourquoi-cantor-texte-de-virginia-hasenbalg

LACAN Jacques, Écrits, Paris, Éditions du Seuil, 1966.

CHABOUD Marc, Girard Desargues, bourgeois de Lyon, mathématicien, architecte, Lyon, 1996, IREM ALÉAS.

PlanCK Max, «Autobiographie Scientifique » 1945, Traduction André GEORGE, Éditions Flammarion, Collection Champs, Paris, 2010.

TATON René, L'œuvre mathématique de Girard Desargues, Paris, 1981, 232 p. 2e éd., Vrin, 1988.

WiGNER E.P., "The unreasonable effectiveness of mathematics in the natural sciences ", Communications on Pure and Applied Mathematics, XIII, 1960, 1-14. 


\title{
Nommer le travail et les travailleurs de la vigne (1860-1960)
}

\author{
Jean-Louis ESCUDIER \\ Chargé de recherche CNRS, \\ Laboratoire montpelliérain d'économie théorique et appliquée (LAMETA), \\ Université Montpellier I, Faculté d’Économie \\ Extrait de : Xavier LAFON, Les langues savantes, Paris, \\ Édition électronique du CTHS (Actes des congrès des sociétés historiques et scientifiques), 2017. \\ Cet article a été validé par le comité de lecture des Éditions du CTHS dans le cadre de la publication \\ des actes du $139^{\circ}$ Congrès national des sociétés historiques et scientifiques tenu à Nîmes en 2014.
}

Les mots du vin donnent lieu depuis deux décennies à pléthore de publications dont le caractère promotionnel prime souvent sur la démarche scientifique. En revanche, le vocabulaire traditionnellement utilisé dans les vignes est désormais négligé. La présente contribution, partie prenante d'un programme de recherche centré sur l'histoire économique et sociale de la viticulture française, tend à poser les premiers jalons d'une investigation approfondie en la matière. Notre champ d'observation privilégie les zones d'expression occitane, mais nous nous référerons également à des termes utilisés dans des régions septentrionales d'expression française (Bourgogne, Champagne) ou germanique (Alsace).

Dans une première partie, nous présenterons la diversité des appellations des travailleurs de la vigne avant d'analyser dans une seconde partie comment la précision terminologique des travaux viticoles et des outils qu'ils requièrent répond à un souci d'efficacité économique.

\section{Du désordre dans la dénomination des travailleurs}

La dénomination des travailleurs offre un paysage lexical d'autant plus complexe que dans chaque microrégion perdure l'usage de termes ancestraux, même lorsque les pratiques culturales connaissent des modifications radicales. Il convient donc de tenter de dénommer chaque catégorie de travailleurs en fonction de sa qualification, de son mode de rémunération (au temps ou à la tâche), de ses avantages en nature.

\section{Le vigneron, un terme pétri d'ambiguïté}

En matière de démographie professionnelle, les apports de la grande enquête des 3000 familles commencée au début des années 1990 (dépouillement par un réseau de généalogistes amateurs de plus de 100000 actes s'étalant de 1790 à 1890) ne furent pas à la hauteur de l'investissement. Pouvait-il en aller différemment tant la terminologie est imprégnée de particularismes locaux qui, souvent, brouillent l'identification d'une profession et de son évolution? Si le «vigneron » arrive au dixième rang des professions en termes d'occurrences, ce qualificatif est loin de réunir la totalité des acteurs viticoles ${ }^{1}$. À partir de la reconstitution postphylloxérique, les propriétaires fonciers méridionaux mirent davantage en avant leur condition de "propriétaire ». Destiné à désigner le

1. J. Dupâquier, J-P. Pélissier, « Mutations d'une société : la mobilité professionnelle », p. 182. 
possesseur d'un bien, ce terme devint alors l'expression d'une condition sociale. Le " propriétaire » est tout à la fois celui possédant un vignoble qu'il exploite en famille et un grand exploitant employant de nombreux salariés. La localisation des 209 vigneronnes répertoriées par l'enquête des 3000 familles $^{2}$ est éloquente : elles résident presque toutes en Lyonnais, en Beaujolais et en Bourgogne, terroirs caractérisés tout au long du $\mathrm{XIX}^{\mathrm{e}}$ siècle par le vigneronnage, mode de faire-valoir fondé sur un partage à mi-fruits entre un propriétaire non exploitant et un couple de vignerons. A contrario, sur les listes nominatives de recensement, les détenteurs d'un contrat de vigneronnage sont indifféremment qualifiés de « vignerons », de « métayer-vignerons » et de « métayer ».

La Champagne fait exception. Au terme d'une réflexion sur «l'épineuse question de la définition $\mathrm{du}$ vigneron champenois ${ }^{3}$, Alexandre Niess conclut que les acteurs de la filière s'accordent pour distinguer les "ouvriers-vignerons », salariés cultivant la vigne pour le compte d'autrui, et les «vignerons ", petits propriétaires exploitants, vinifiant parfois eux-mêmes mais vendant le plus souvent leurs raisins à un négociant-éleveur.

Aux spécificités régionales se surajoute un traitement très différencié selon le genre. En bien des lieux, seuls les hommes cultivant la vigne sont qualifiés de vignerons ou d'ouvriers. Parmi d'autres exemples, citons cet ouvrage rédigé en 1865 par une kyrielle de sommités agronomiques signalant que la culture du vignoble médocain mobilise alors « 9000 vignerons, 9000 femmes et 1500 terrassiers » ${ }^{4}$. Selon le sous-préfet de Saint-Pons dans l'Hérault, en 1906, le domaine de Sériège, près de Cruzy emploie « 47 journaliers et 27 femmes $»^{5}$. Dans son Dictionnaire du monde rural, Marcel Lachiver ne retient pas la même définition pour les hommes que pour les femmes. Le vigneron est:

«Celui qui cultive la vigne et élabore le vin et que l'on voudrait aujourd'hui appeler viticulteur $»^{6}$

et

« celui qui, en Mâconnais, cultive la vigne à moitié-fruits. »

La seconde acception du terme « vigneronne » est « la femme qui cultive la vigne ${ }^{7}$. En se féminisant, la fonction s'est restreinte, sans la moindre explication, au seul espace de la vigne : à défaut de formulation explicite, le lecteur doit déduire de ces définitions que l'élaboration du vin est réservée au seul vigneron.

Nous nous sommes attaché, pour les principaux départements viticoles, à dénombrer les "vignerons » dans l'enquête de 1929, première des grandes enquêtes agricoles du $\mathrm{XX}^{\mathrm{e}}$ siècle $^{8}$. À en croire les données publiées, le territoire national aurait compté 156207 vignerons à la fin de l'année 1929. Pour aussi précis qu'il soit, ce nombre minore et déforme très fortement la réalité viticole. L'absence complète ou presque de « vignerons » dans des départements éminemment viticoles tels la Côte-d'Or, la Saône-etLoire, la Charente-Inférieure et les Bouches-du-Rhône prouve que, d'un département à l'autre, les maires interrogés n'ont pas retenu les mêmes critères pour répartir les individus contribuant à la production viticole. Manifestement, des individus classés ailleurs comme "vignerons » furent recensés dans le Gard et la Charente-Inférieure dans la catégorie «autres personnes... » puisque ces deux catégories y sont pléthoriques. Certaines commissions communales et cantonales comptabilisèrent dans cette catégorie une multitude d'individus qu'un examen un tant soit peu attentif aurait permis d'affecter à une catégorie plus explicite. Dans quelques départements, cette catégorie «autres

2. C. Motte, J-P. Pélissier, «La binette, l'aiguille et le plumeau : les mondes du travail féminin », p. 303.

3. A. Niess, "Champagne rouge, champagne sang. De l'épineuse question de la définition du vigneron champenois (1908-1914)», p. 97-111.

4. C. Alibert, « Des vignes du Médoc », p. 250.

5. Archives départementales de l'Hérault, 10 M 280, Lettre du sous-préfet de Saint-Pons au préfet de l'Hérault, 18 octobre 1906.

6. M. Lachiver, Dictionnaire du monde rural. Les mots du passé, p. 1692.

7. Ibid, p. 1692.

8. Ministère de l'Agriculture, Direction de l'Agriculture, Statistique agricole de la France. Résultats généraux de l'enquête de 1929, Paris, Imprimerie Nationale, 1936, p. 534-537. 
personnes » comprend plus de la moitié de la main-d'œuvre; le cas de la CharenteInférieure, où $70 \%$ des individus recensés furent affectés à cette catégorie, est caricatural.

\section{La dénomination des salariés viticoles : une palette riche et tout en nuances}

Le prolétariat viticole constitue un ensemble composite, aux contours difficiles à cerner en raison d'une diversité des statuts professionnels et des conditions sociales. Au début $\mathrm{du} X \mathrm{X}^{\mathrm{e}}$ siècle, cette classe ouvrière se subdivise schématiquement en deux groupes économiquement, socialement et culturellement nettement différenciés : les journaliers d'une part, et les gagés d'autre part.

Au premier rang des travailleurs de la vigne se situe le «journalier». Comme son nom l'indique, celui-ci est l'homme employé et rémunéré à la journée. Sous l'Ancien Régime, il se situe généralement sous le manouvrier qui peut posséder quelques parcelles et il constitue la main-d'œuvre des gros labours des grandes plaines à blé. Dans les régions moins riches, l'écart entre le journalier et le laboureur est moins marqué, le terme de «laboureur » pouvant même désigner l'homme de labeur. Le «volant» était, dans le Midi méditerranéen, un ouvrier viticole louant ses services à la journée pour différents propriétaires. Ce salarié percevait de 10 à $20 \%$ de plus que les journaliers attachés à un seul et même employeur : cette prime était censée rémunérer le risque de la précarité de l'emploi accepté par ces ouvriers, généralement habiles et rapides.

Rémunérés à l'année selon des modalités proches de celles pratiquées en polyculture/élevage, les ouvriers gagés embauchés en viticulture furent dénommés "domestiques » tout au long du XIX ${ }^{\mathrm{e}}$ siècle et, en bien des contrées, jusqu'à la Seconde Guerre mondiale. Le salaire en nature (gîte et couvert) prend alors nettement le pas sur le salaire en espèces, lui-même généralement modulé selon les saisons et l'intensité des travaux requis ; ces domestiques sont généralement des hommes célibataires. Employé au féminin, le terme «domestique » est ambigu, car il s'appliquait aussi bien à la fille de ferme qu'à l'employée de maison. Les femmes étant occupées tant dans la sphère domestique que dans la sphère professionnelle, dans bien des cas, la césure entre ces deux espaces de travail était ténue, voire inexistante. Les mésadiers étaient rémunérés au mois et souvent embauchés pour une partie de l'année. Célibataires ou hommes mariés résidant le reste de l'année dans des contrées non viticoles, ces salariés étaient également nourris et logés.

Sans être aucunement spécifique au pays de vignobles, au XIX ${ }^{\mathrm{e}}$ siècle, le terme de " brassier» se retrouve dans plusieurs terroirs. Son étymologie transparente (homme qui travaille avec ses bras) laisse à penser qu'il désignait des salariés dépourvus de qualification, mais il n'est pas pour autant synonyme de l'expression «homme (ou ouvrier) toutes mains", utilisée dans certains contrats de travail et conventions collectives jusqu'aux années 1960. Le brassier peut manipuler un outil (bêche, pioche, râteau...) mais ne se voit pas confier un attelage et encore moins une machine ou un tracteur. Il est souvent opposé au laboureur, travailleur qualifié effectuant les labours mais aussi toutes les tâches impliquant la conduite d'animaux de trait.

$\mathrm{Au} X \mathrm{X}^{\mathrm{e}}$ siècle, le terme $\mathrm{d}^{\prime}$ " ouvrier agricole» apparaît dans la viticulture méridionale, mais il resta longtemps limité aux exploitants ayant suivi une école d'agriculture. Les rédacteurs des listes de dénombrement établies dans le cadre des recensements quinquennaux de la population, lesquels, en milieu rural, furent les directeurs d'école faisant office de secrétaire de mairie, optèrent plus souvent pour "cultivateur », terme suffisamment vague pour englober des ouvriers démunis de toute propriété foncière, des métayers et la myriade de salariés détenteurs d'un vignoble plus ou moins important.

Ces recensements de la population ne sont d'ailleurs pas d'un grand secours pour faire émerger une quelconque logique dans la dénomination des salariés. À titre d'illustration, nous considérerons une très grande exploitation agricole et viticole méridionale, le domaine de l'étang asséché de Marseillette, au cœur du département de l'Aude : 820 
hectares d'un seul tenant dont 340 de terres labourables, 225 de vignes et 80 de luzernes s'étendant sur cinq communes contiguës. Au début des années 1930, la propriété emploie 160 salariés «des deux sexes ». La profession attribuée à ces salariés lors des recensements généraux de la population de 1926, 1931 et 1936 est extrêmement disparate selon leur commune de résidence. À Puichéric, les hommes sont qualifiés de «cultivateurs" alors que les jeunes filles sont « ouvrières agricoles ». Dans les deux domaines situés sur la commune de Marseillette, les hommes et leurs fils en âge de travailler sont "ouvriers agricoles», les femmes qualifiées de "sans profession ». À Saint-Frichoux, les hommes sont enregistrés comme "cultivateurs », les jeunes filles sont dénommées «cultivatrices". Alors que tous ces salariés sont employés dans les mêmes conditions salariales, ils font l'objet de classifications professionnelles diverses au gré de la perception du secrétaire de mairie de leur lieu de résidence. Chacun utilise les expressions lui paraissant les plus appropriées sans se soucier de s'inscrire dans une typologie commune que les services préfectoraux n'avaient d'ailleurs pas conçue. Toutefois, une constante demeure : les femmes salariées sur l'exploitation ne bénéficient pas du même traitement lexical que leurs homologues masculins.

\section{Des dénominations traduisant des rapports de genre inégalitaires}

La qualité de «travailleur agricole » est réservée au genre masculin. L'asymétrie des définitions retenues par $\mathrm{M}$. Lachiver prête à sourire. L'ouvrier y est décrit comme une " personne qui œuvre, qui travaille, surtout pour autrui. Ouvrier agricole ». En revanche, la seule acception retenue pour expliciter le substantif féminin " ouvrière " a trait aux abeilles ${ }^{9}$. Cet auteur procède de même pour les termes «journalier », logiquement défini comme un « homme qui travaille à la journée », et «journalière », dont la seule acception retenue renvoie à la dénomination d'une petite herse en Anjou ${ }^{10}$. Enfin, si le cultivateur est " celui qui cultive la vigne ${ }^{11}$, la cultivatrice $n^{\prime}$ a pas droit de cité dans ce dictionnaire. La très riche iconographie de cet imposant ouvrage suffirait pourtant à attester la présence active d'ouvrières agricoles dans toutes les régions et toutes les productions végétales ou animales.

Une autre pratique terminologique traduit le refus de reconnaître aux ouvrières viticoles l'exercice d'un métier au sens complet du terme : leur désignation en fonction de la tâche réalisée selon les besoins de l'heure. En les qualifiant de sarmenteuses, de lieuses, de couvreuses pour celles effectuant les opérations connexes au greffage, de vendangeuses ou encore de ciseleuses lors de l'apprêtement des raisins de table, les monographies agricoles mettent l'accent sur l'absence de statut économique des salariées viticoles et sur la précarité de leur emploi. En la matière, l'asymétrie avec les dénominations des travailleurs masculins est totale. En effet, la spécification de leurs fonctions viticoles (laboureurs, porteurs de hotte, greffeurs...) signifie de facto la reconnaissance d'une qualification, l'octroi d'un salaire majoré et un emploi pérenne.

Dans la plupart des grands domaines viticoles méditerranéens, le propriétaire délègue la direction effective de l'exploitation à un régisseur, fondé de pouvoir ou chargé d'affaires, généralement payé à l'année et intéressé aux bénéfices de l'exploitation. Chaque matin, le régisseur donne ses consignes au mousseigne, contremaître assurant le rôle de chef d'équipe des journaliers et à la mousseigne, responsable de l'équipe des journalières. Bien que rarement explicitée, cette organisation repose sur une hiérarchie de fait: le chef de l'équipe des hommes est généralement un maître-valet, et à ce titre "numéro deux » de l'exploitation alors que la mousseigne des journalières est elle-même journalière.

Le maître-valet, dénommé ramonet dans l'Aude et l'Hérault, païre dans l'Hérault, baïle dans le Gard, granger en Roussillon et dans le Var, est un ouvrier qualifié : rémunéré au mois, il est chargé de l'entretien des chevaux et fait office de chef d'équipe. Mais ce salarié doit obligatoirement être marié car son épouse (dénommée selon les lieux

9. M. Lachiver, Dictionnaire du monde rural. Les mots du passé, p. 1229.

10. Ibid., p. 996.

11. Ibid., p. 596 
ramonette, maïre ou baïlesse) joue un rôle essentiel dans l'organigramme des grandes exploitations viticoles. Cette femme nourrit les domestiques engagés à l'année et entretient le potager et les petits élevages (poulailler, clapier, pigeonnier...). Dans certains cas, elle facture au propriétaire les denrées achetées mais, le plus souvent, elle doit jongler avec une somme fixe trop restreinte pour assurer des repas corrects et suffisamment abondants, et, éventuellement, dégager un excédent correspondant à sa rémunération ${ }^{12}$.

En Camargue, où les domestiques logés et nourris constituent l'essentiel de la maind'œuvre, la plupart des grands domaines viticoles affectent à ce service de la nourriture une cuisinière et son aide, localement dénommées tante et tanton ${ }^{13}$. Cette question de la nourriture est cruciale, car les ouvriers nourris y attachent une importance primordiale. En bien des cas, leur implication dans les travaux de l'exploitation en dépend. Par les responsabilités qui lui incombent, cette femme assure donc sans conteste une fonction relevant de la maîtrise. Si ses compétences sont appréciées, en revanche, cette qualification de contremaîtresse ne lui est jamais reconnue. Pour être spécifique, son cas ne déroge pas à la loi commune de la précarité et de l'absence de reconnaissance de qualification de la main-d'œuvre agricole féminine.

\section{La précision terminologique, gage d'efficacité économique}

Les termes régionaux ou locaux visent à la précision des fonctions que le français académique ne peut exprimer que par une périphrase. Ils ont, entre autres fonctions, celle de désigner des outils, des espaces ou des situations de travail que la langue officielle ne distingue pas. Au travers d'un survol des unités de mesure utilisées en pays viticole et de l'examen de deux principales fonctions viticoles, à savoir la taille d'hiver et la vendange, nous analyserons le degré de cohérence de cette diversité terminologique.

\section{La persistance des unités de mesure locales anciennes}

Si l'instauration du système métrique n'a pas fait disparaître ipso facto, tant s'en faut, l'usage des anciennes unités de mesure, il serait fallacieux d'y voir le seul produit du caractère routinier des paysans. En effet, les mesures de quantité et de surface sont intimement liées à un processus de production, à des normes de productivité. Ainsi, le journal (parfois modifié en jour, journeau, journée ou journel), terme générique désignant la surface qu'un homme peut travailler dans une journée, se retrouve dans toute la France. Dans plusieurs contrées vigneronnes, du Beaujolais en Franche-Comté en passant par la Bourgogne, le terme ouvrée ou œuvrée (dérivé du substantif « œuvre ») eut la faveur des viticulteurs pour désigner la surface ou le nombre de ceps de vigne travaillés par un vigneron durant une journée. Toutefois, dans certains villages du Beaujolais, on employait le terme hommée. En Bordelais, le journal, qui se divise en règes, carreaux, brosses, onces, lattes ou escats, varie de 27 à 47 ares. À Bergerac (Dordogne), le journal vaut 33,73 ares. Spécifique au milieu viticole est la rège (ou ses variantes règue, reige, rèse). Désignant dans sa première acception le rang de vigne, la rège devint en Gironde viticole une unité de superficie utilisée faisant de $1 / 6^{\mathrm{e}}$ à $1 / 32^{\mathrm{e}}$ de journal et comptant dix pas carrés, soit $79 \mathrm{~m}^{2}$. Toujours en Médoc, le système de mesure était complété par le sadon valant 410 règes de cent pas carrés représentant 7,90 ares. Il y avait quatre sadons dans un journal de 31,60 ares et l'hectare était compté pour trois journaux ou douze sadons.

Cet ensemble métrologique peut aujourd'hui paraître abscons mais il était fort simple aux yeux des viticulteurs, qu'ils soient propriétaires fonciers, fermiers ou salariés. Ce n'est pas la superficie, notion abstraite, qui importait mais le travail concret effectué par un individu à l'œuvre dans une vigne. D'ailleurs, la rège a donné en Médoc le régéage, terme

12. J.-L. Escudier, Contribution à l'histoire des rapports économiques de genre. Application à la viticulture française, 1850-2010, p. 145-146.

13. J. Vigouroux, Essai sur le fonctionnement économique de quelques très grandes exploitations dans la Camargue et le Bas-Languedoc, p. 109. 
désignant le chaussage des vignes. Si ces mesures agraires ont officiellement disparu, il y est régulièrement fait appel non seulement dans le cadre restreint de l'exploitation mais encore dans les documents réglant les relations entre acteurs sociaux. Ainsi, la convention collective des exploitations viticoles de la Côte-d'Or, conclue le 27 juin 1952, stipule que "l'unité de superficie retenue comme base du calcul pour la détermination des salaires à la tâche est le journal (8 ouvrées) soit 34 a 24 ca » ${ }^{14}$.

\section{Identifier finement le terroir, les outils et le travail}

L'identification précise de l'espace viticole et du travail qu'il requiert est primordiale à toute exploitation bien comprise. Ainsi, le rang de vigne plus court que la majorité de ceux qui composent l'ensemble de la parcelle est-il appelé «cabot» en Médoc ${ }^{15}$. Le cavaillon, dont l'usage est encore patent de nos jours dans la plupart des vignobles, désigne la bande de terre d'accès difficile large d'environ quarante centimètres située sous le rang de vigne et en particulier autour de la souche où elle fait butte, qu'on creuse au printemps en décavaillonnant pour aérer les souches, mettre le fumier ou l'engrais, détruire les parasites. Ce terme était si utile à l'exploitant viticole que la mécanisation non seulement n'en précipita pas l'obsolescence mais fut l'occasion d'en étendre l'usage puisque les engins aratoires conçus pour cultiver mécaniquement cet espace aussi spécifique furent dénommés « décavaillonneuses ».

Le déchaussage désigne l'opération consistant, le printemps venu, à enlever la terre autour des souches sur une profondeur de vingt à trente centimètres. Mais le terme occitan escausselar, perçu comme plus parlant, fut souvent francisé en "échausseler ». Ainsi, que l' «escaussel » désigne tout à la fois la pioche spécialement conçue pour déchausser la vigne et l'excavation résultant du déchaussage ne générait aucune confusion; cette polysémie n'entravait en rien la précision terminologique. Pour l'exploitant, ce qui importait était la désignation et par là même l'individualisation de la tâche requise et sa compréhension par le personnel d'exécution.

En Languedoc, en matière de greffage, on distinguait les greffeurs proprement dits, les tuteleurs et les couvreuses. La vigneronne qui explique le contenu de ce travail ressent le besoin de détailler encore pour bien se faire comprendre de son interlocutrice :

«Il y a le greffeur, l'homme qui met le tuteur et puis la femme qui abousole, qui amotte quoi. $»^{16}$

Dans le même ordre d'idées, l'individu remplaçant les plants de vigne n'ayant pas pris ou les souches mortes, était dénommé le remandaire. De même, le quitchaire était l'homme relativement âgé, ne pouvant plus assurer le portage des raisins, qui tassait le raisin dans les comportes à l'aide d'une masse en bois et, au besoin, répartissait au mieux le contenu afin d'homogénéiser le chargement; on employait à ce propos l'expression "faire les comportes ». Ainsi, loin d'être superfétatoire, la précision terminologique des fonctions viticoles relève d'une exigence d'efficacité tant dans la commande de certains travaux que dans l'évaluation du temps de travail nécessaire.

\section{La taille d'hiver ou la confusion des outils}

Cette fonction viticole réalisée durant l'hiver comprenait historiquement deux volets bien distincts, à savoir la taille proprement dite et le ramassage des sarments coupés.

La taille de la vigne était ancestralement effectuée dans tous les terroirs au moyen d'une serpe, appelée en occitan poda, podet ou podadore. Au cours de ses investigations dans l'iconographie médiévale, Perrine Mane n'a identifié que deux œuvres mettant en scène

14. Journal officiel de la République française du 28 octobre 1953, p. 9676-9677.

15. A. Viaut, F. de vinha, En Médoc, paroles d'oc du vignoble à Saint-Estèphe, p. 115.

16. C. Amiel, G. Charuty, C. Fabre-Vassas, Jours de vigne. Les femmes du pays viticole racontent le travail, p. 37. 
une femme en train de tailler la vigne ${ }^{17}$. Cet homme taillant la vigne est logiquement dénommé poudaire mais, signe de la primauté accordée à cette tâche, ce substantif tend à qualifier plus généralement tout travailleur de vigne. L'onomastique témoigne également de la noblesse de la fonction: Poudevigne reste un nom propre répandu dans tout l'espace occitanophone. À partir de 1817, est ponctuellement utilisé dans le Midi méditerranéen un outil à deux lames tranchantes «ayant la forme d'un forceps [...] pour nettoyer les ceps et séparer le bois mort ${ }^{18}$. Ce nouvel outil, qui ne porte pas encore le nom de sécateur, mettra du temps à chasser la serpe du paysage viticole.

Nombre de confusions ultérieures trouvent leur origine dans une imprécision terminologique puisque le substantif «sécateur» fut durablement employé pour désigner indistinctement les sécateurs proprement dits, actionnés d'une seule main, et les ciseaux à tailler la vigne, lesquels s'apparentent plutôt à la famille des cisailles et mobilisent les deux mains de l'opérateur. En décrivant la serpe utilisée en Médoc comme un outil qui «remplit les fonctions de sécateur, de hache et même de petite scie ${ }^{19}$, C. Alibert participe en 1865 de la confusion sémantique ambiante. Dans le Traité d'agriculture à l'usage du Midi de la France publié en 1893, les ciseaux à tailler sont figurés sous la légende "Sécateur à vigne du Languedoc $»^{20}$. Robert Herman soutient que le ciseau à tailler, qu'il qualifie de "sécateur coupe-ceps", aurait remplacé à la fin du XIX siècle la scie égoïne et la serpe à dos tranchant car :

«Grâce à son manche relativement long et la robustesse de ses deux lames, [il] permet de couper facilement et rapidement les bois morts. $»^{21}$

Cette configuration correspond probablement à l'évolution constatée en certains terroirs mais elle est loin d'être générale : scie égoöne et ciseaux à tailler formèrent jusqu'aux années 1970 la panoplie du tailleur de vigne languedocien. Plus fondamentalement, nous ne sommes pas parvenus à dégager de la collection Herman une chronologie un tant soit peu fiable de l'usage du large éventail des outils tranchants destinés à la taille d'hiver ${ }^{22}$.

Sur une souche, la branche à fruit conservée plus ou moins longue et courbée en demicercle, dénommée en français "aste", reçut selon les régions les noms les plus divers : courgée, vinée, pleyon, sauterelle, flèche, tiret, dague, arçon, arceau. Elle s'oppose au cot de retour qui fournira le bois de la taille de l'année suivante. Lorsqu'il s'agit d'une branche à fruit supplémentaire conservée afin d'accroitre la production d'une souche, cette «aste " s'est vue donner le nom très évocateur de pisse-vin en Médoc comme en Languedoc.

Dans tous les terroirs viticoles français, les sarments furent durablement ramassés par des femmes, salariées ou épouses de petits exploitants. Réunis en fagots bien ordonnés, ces sarments alimentaient les cheminées de la demeure du propriétaire et, selon les usages locaux, la «sarmenteuse » salariée se voyait accorder un certain nombre de fagots pour sa consommation familiale. Le reliquat était vendu soit pour un usage domestique urbain soit pour alimenter les fours de boulangers. Les sarments, ramassés tous dans le même sens, étaient rassemblés par poignées de dix à douze et liés par un long sarment enroulé en spirale. Huit ou dix de ces poignées (la moitié disposée dans un sens, l'autre moitié dans l'autre) formaient un fagot, lui-même attaché par un brin d'osier, plus rarement par un fils de fer. Dans l'Aude, la buffanela était un fagot fait de sarments toujours ramassés dans le même sens (c'est-à-dire tous les pieds tournés du même côté). Dans le Biterrois, au contraire, la buffanela désignait un fagot de sarments ramassés en vrac.

17. P. Mane, « Vigneronnes et vignerons dans l'iconographie médiévale », p. 19.

18. R. Pech, «Quelques réflexions à propos de la taille de la vigne en Languedoc méditerranéen aux XIX ${ }^{\mathrm{e}}$ et $X X^{\mathrm{e}}$ siècles $»$, p. 83 .

19. C. Alibert, « Des vignes du Médoc », p. 250.

20. B. Chauzit, J.-B. Chapelle, Traité d'agriculture à l'usage du Midi de la France, p. 154, figure 58.

21. R. Herman, Anciens outils de la vigne et de la tonnellerie, p. 88.

22. Ibid., p. $79-88$ 
Selon les terroirs, le ramassage des sarments a donné lieu à une profusion de termes. Pour le seul espace occitanophone, on relève : ramasser ou lever les bûches, faire, tomber ou tirer les bois, faire les sarments, et en certains lieux gaveler, directement issu de l'occitan gavel. Pour R. Falissard, le gavèl (ou gaveu) serait la botte de sarments composée de douze javelles ou poignées de sarments ${ }^{23}$ alors que pour d'autres auteurs, ce terme désigne un seul et unique sarment.

Le temps de la taille se termine par la pose des échalas car, lors du développement de ses sarments, entre les mois d'avril à août, la vigne en croissance aura besoin d'être soutenue. Cette fonction, aujourd'hui assurée par des fils de fer, fut longtemps réalisée par un système plus ou moins complexe d'échalassement. Cette tâche était désignée en Bourgogne viticole par le terme paisselage ou empaisselage. Ces piquets de bois pour soutenir la vigne ont donné lieu à une grande diversité lexicale : paisseaux en Bourgogne, carassons en Bordelais, paissel, passel, paxel dans le Midi méditerranéen, pessiau en Chalonais et en Bresse ou tout simplement échalas en Champagne. L'échalas de quartier était en cœur de chêne ou en châtaignier, bois peu putrescibles. Il était alors distingué de l'échalas de pied, en aubier de chêne. Les dimensions des échalas étaient variables d'un terroir à l'autre selon la façon de tailler la vigne : 1,33 à 1,56 m en Bourgogne, 1,50 à $1,65 \mathrm{~m}$ en Gironde, 2,5 à $3 \mathrm{~m}$ en Alsace et dans le Jura pour la conduite des hautains. Les échalas étaient plantés avec un maillet, à la planchette, c'est-à-dire en pesant de tout son corps au moyen d'une planchette de bois attachée sur la poitrine, avec des fiche-échalas, ou encore à l'aide d'une clé-ficheuse. Enfin, le secaillage désignait, en Médoc, l'opération consistant à collecter à l'orée de l'hiver les vieux échalas hors d'usage (appelées secailles).

\section{Une diversité de modes opératoires durant les vendanges}

Aboutissement du travail de toute une année, la vendange est un temps fort et crucial qu'il convient de bien négocier afin de rentrer dans les meilleures conditions les raisins destinés à élaborer le vin. La diversité des pratiques répond à des contraintes topographiques (déclivité, taille des parcelles, éloignement des chais...) et climatiques (chaleur, humidité...) et à des exigences œnologiques (rendement, degré de maturité...).

L'équipe de vendangeurs était diversement identifiée selon les régions: hourdon ou hordon en Champagne, troupe en Bordelais, colle (de l'occitan colha) dans le Midi méditerranéen et le Sud-ouest. L'une des coupeuses était chargée de rythmer la cueillette; généralement apparentée au propriétaire ou au régisseur de l'exploitation, cette femme, dénommée menaira ou capitèna dans l'Hérault, menaida ou bailesa, dans le Gard et la basse vallée du Vidourle, musseigna dans l'Aude et les Pyrénées-Orientales, réglait l'allure du travail en coupant les raisins dans la première rangée, deux ou trois souches en avant des autres. Si l'organisation du travail de cette équipe plus ou moins fournie selon la dimension de l'exploitation est générale, il n'en a pas résulté une harmonisation des pratiques. Au risque de schématiser quelque peu, nous considérerons deux phases essentielles dans l'opération de vendange: la cueillette des raisins proprement dite et leur acheminement en bordure de la vigne, d'une part, et le charroi de la vigne au chai, d'autre part.

L'usage de la hotte se répandit dans presque tous les terroirs viticoles français sans être exclusif. Selon les régions, on relève plusieurs types de hotte ${ }^{24}$. Très profonde en Alsace et en Moselle, la hotte traditionnelle était moins profonde et plus évasée en Languedoc. À la hotte originelle, en bois, se substituera à partir de l'entre-deux-guerres la hotte en fer, elle-même remplacée vers 1960-1970, par la hotte en plastique, nettement plus légère. Le vendangeur portant la hotte est dénommé porteur dans le midi de la France (en occitan gorpéjaire), hotteur en Beaujolais, hostier ou hottier en Bourgogne et porte-hotte en

23. R. Falissard, Vignes, vins du pays d'Oc. Vocabulaire de la langue d'Oc concernant la culture, la vinification, les instruments et la boisson suivi des dictions et proverbes (avec définitions et explications en français), p. 134.

24. Plusieurs modèles de hotte sont figurés dans M. Lachiver, Dictionnaire du monde rural. Les mots du passé, p. 1009-1011. 
Gironde. La hottée (ou hottelée en Chalonnais) désigne le contenu variable d'une hotte de vendange.

Ignorant la hotte, certaines contrées eurent longtemps recours aux services de videurs de seaux sur le pourtour méditerranéen et de vide-paniers en Gironde. En Languedoc, le domaine de la hotte recouvrait les zones de coteaux aux rendements limités (40 à $60 \mathrm{hl} / \mathrm{ha}$ ). Dans les plaines fertiles et irrigables où prévalait l'aramon, cépage gros producteur (entre 100 à $150 \mathrm{hl} / \mathrm{ha}$ ), les videurs de seaux apparurent plus efficaces qu'un porteur de hotte n'ayant matériellement pas le temps d'effectuer le trajet aller-retour avant que les seaux ne soient déjà remplis. Toutefois, la force de l'habitude semble avoir été prépondérante, car ces façons de faire n'étaient pas remises en cause même dans les vignes à haut rendement des terroirs de coteaux ou, a contrario, quand, pour une cause ou une autre, la récolte était médiocre en plaine. Le lexique viticole du Biterrois de Jean-Marie Petit livre détaille en français et en occitan l'organisation de la vendange dans cette « mer de vigne » bas-languedocienne ${ }^{25}$.

La partition géographique entre hotte et videurs de seaux s'estompera quelque peu sous l'effet de la mécanisation. Les rédacteurs de l'Atlas linguistique du Languedoc oriental insistent sur la transformation à l'œuvre au cours des années 1970 :

«La hotte de vendange s'est répandue un peu partout, en même temps qu'apparaissaient les bennes automotrices. Au lieu de l'ancien système dans lequel le seau individuel du coupeur était vidé dans la comporte que deux hommes portaient sur la charrette. ${ }^{26}$

Mais cette nouvelle organisation sera elle-même rendue obsolète lorsque la machine à vendanger s'imposera dans la plupart des terroirs.

Dans le Montpelliérais et les basses plaines gardoises, on utilisait le banastou, petite comporte à vendange d'une contenance de 20 à 25 litres, transportée à dos d'homme. Contrairement aux comportes, ce banastou n'était pas placé directement sur la charrette pour être acheminé jusqu'au chai, mais transvasé dans un tombereau, localement dénommé pastière. En Champagne, en Mâconnais et en Beaujolais, le raisin coupé était acheminé à l'extérieur de la vigne au moyen du jarlot (parfois dénommé jerle, jarle, jalot ou encore jalais). Récipient doté le plus souvent de deux poignées, son contenu était versé dans un panier d'osier oblong contenant de 20 à $50 \mathrm{~kg}$ de raisin: le benon (ou ses variantes bénaton, bénéton ou benneton). Dans les plus prestigieux des vignobles champenois, la vendange proprement dite se doublait d'une opération de tri in situ. Attablées en bordure de vigne, de dix à vingt femmes, dénommées "éplucheuses ", expurgeaient chaque grappe des grains avortés ou gâtés.

Le charroi du raisin de la vigne au chai s'opérait selon deux grandes modalités: la balonge ou la comporte. La balonge (parfois dénommée balange, barlon ou ballote), sorte de cuvier rond ou ovale fixé à demeure sur une charrette, eut la faveur des vignobles septentrionaux. En Bourgogne et dans le Jura, la balonge est une cuve large, sans poignées, d'un diamètre approximatif de $1,5 \mathrm{~m}$ et de $0,85 \mathrm{~m}$ de hauteur. En Champagne, il était plutôt de coutume de positionner deux cuves sur le même plateau de charrettes, et en Alsace, trois cuves: par voie de conséquence, ces récipients étaient de dimension moindre. En Languedoc, l'instrument le plus répandu pour transporter le raisin de la vigne au chai fut la comporte (semal en occitan). L'usage de ce récipient fut si commun durant les années 1880-1950 qu'elle devint unité de mesure. Un propriétaire viticole ne comptabilisait pas la production de ses vignes en kilos ou en tonnes mais en comportes, le poids de celle-ci, raisonnablement remplie, étant estimé à $80 \mathrm{~kg}$.

La coutume de fêter la fin de vendange par un repas de fête offert par l'employeur se retrouve dans tous les terroirs viticoles, mais les appellations de ces agapes sont aussi

25. J-M. Petit, Le lexique viticole du Biterrois, p. 160-168.

26. J. Boisgontier (dir.), L'Atlas linguistique et ethnographique du Languedoc oriental, N872a: Hotte (Gorp), $872 \mathrm{~b}$ : benne (pastiera). 
multiples que les modalités qu'elles revêtent. Sans être exhaustif, citons gerbaude ou gerbebaude en Médoc et en Dordogne, acabailles dans le Sauternais, pampaillet dans les Graves de Bordeaux, paillou en Saintonge, manger l'oie en Touraine, Dius a vol en Corbières et en Narbonnais, soulinque en Biterrois, revole ou paulée en Beaujolais et en Mâconnais, poêlée en Sancerrois, tue-le-chien en Côte-d'Or, dans le Jura et en pays messin, cochelet en Champagne, Herbschtbrote en Alsace.

Le lexique viticole a pour fonction première de déterminer précisément un lieu, un espace à l'intérieur de la vigne, un outil adapté à un travail déterminé. La mécanisation d'une fonction viticole ne se traduit pas toujours par une rupture lexicale. On a souvent affublé un engin motorisé du terme précédemment utilisé pour qualifier une tâche manuelle. Mais le lexique viticole participe également de la cohésion sociale d'un groupe restreint. Les conventions collectives viticoles conclues à partir de 1950 et périodiquement remises à jour s'inscrivent dans le cadre aussi vaste qu'incertain des " usages locaux ». Certaines d'entre elles font d'ailleurs référence à d'ancestrales unités de mesure de temps ou de surface (journal, ouvrée, coupée, sadon...), à une terminologie purement locale pour dénommer fonctions (ramonet, païre, baile, granger, prix-faiteur...) et travaux viticoles (évasivage, relevage...).

À l'évidence, le statut social et le niveau de formation des individus influent sur la lexicologie viticole. Les viticulteurs formés dans les écoles d'agriculture acquirent un vocabulaire professionnel moins influencé par les dialectes locaux. Mais, la communication avec les salariés formés sur le tas ou sans qualification les obligeait à recourir au quotidien au vocabulaire traditionnel de leur terroir.

Enfin, les différents modes d'acquisition des savoirs viticoles n'ont pas encore généré une langue viticole unique. Il serait très réducteur sinon fallacieux d'évoquer les pratiques ou le mode de vie d'une communauté vigneronne. En réalité, coexistent une multitude de micro-communautés de vignerons, chacune d'entre elles pratiquant un jargon professionnel qui n'est pas immédiatement accessible aux vignerons d'autres terroirs. Cette diversité s'explique en partie par des considérations d'ordre climatique, topographique et économique. Toutefois, des terroirs viticoles comparables et peu éloignés révèlent un corpus linguistique profondément différent. Ces zones de fracture traduisent une réalité socioculturelle: loin d'être réductible à un simple outil de communication, le langage viticole contribue à forger une identité collective, donc in fine, le terroir. Ainsi, la terminologie revêt une forte composante patrimoniale dont attestent, dans toutes les contrées viticoles de France, les multiples musées de la vigne et du vin. La transformation radicale des facteurs de production et de l'organisation du travail viticole à l'œuvre depuis une génération justifierait à elle seule la mise en place d'une vaste enquête orale visant à préserver une richesse lexicale en voie d'extinction. 


\begin{abstract}
Résumé
Le vocabulaire traditionnellement utilisé dans les vignes est désormais largement supplanté par l'engouement dont bénéficient les lexiques œnologiques. Partie prenante d'un programme de recherche centré sur l'histoire économique et sociale de la viticulture française, cet article tend à poser les premiers jalons d'une investigation sur les termes viticoles et leur degré de rationalité. Notre champ d'observation privilégie les zones d'expression occitane mais nous nous référerons également à des termes utilisés dans des régions septentrionales d'expression française (Bourgogne, Champagne) ou germanique (Alsace).

Nous nous attachons, dans une première partie, à présenter la diversité des appellations des travailleurs de la vigne avant d'analyser dans une seconde partie comment la précision terminologique relative aux travaux viticoles et aux outils qu'ils requièrent répond à un souci d'efficacité économique.
\end{abstract}

\title{
Bibliographie
}

Alibert C., « Des vignes du Médoc » dans JoigneAux Pierre (dir.), Le Livre de la ferme et des maisons de campagne par une réunion d'agronomes, de savants et de praticiens, Paris, Victor Masson et fils, F. Tandou et Cie, 1865, p. 242-266.

AmIEL Christiane, CHARUTY Girodana, FABRE-VASSAS Claudine, Jours de vigne. Les femmes du pays viticole racontent le travail, Villelongue-d'Aude, Atelier du Gué, 1981.

BOISGONTIER Jacques, (dir.), L'Atlas linguistique et ethnographique du Languedoc oriental, Paris, 1981-1986, 3 vol. Éd. du CNRS.

Chauzit B. et CHAPElle J.-B., Traité d'agriculture à l'usage du Midi de la France, Montpellier, Camille Coulet libraire-éditeur, Paris, G. Masson, 1893.

DUPÂQUIER Jacques et PÉLISSIER Jean-Pierre, "Mutations d'une société : la mobilité professionnelle » dans DUPÂQUIER Jacques et KESSLER Denis, (dir.), La société française au XIX ${ }^{e}$ siècle. Tradition, transition, transformations, Paris, Fayard, 1992. p. 124-235.

ESCUDIER Jean-Louis, Contribution à l'histoire des rapports économiques de genre. Application à la viticulture française, 1850-2010, thèse de doctorat en histoire et histoire de l'art, Université de Perpignan Voie Domitienne, 2014, 2 vol.

FALISSARD Renaud, Vignes, vins du pays d'Oc. Vocabulaire de la langue d'Oc concernant la culture, la vinification, les instruments et la boisson suivi des dictions et proverbes (avec définitions et explications en français), Rodez, Lo Grelh roergàs, 2002.

HERMAN Robert, Anciens outils de la vigne et de la tonnellerie, avant-propos de Claude ROYER, Grenoble, Glénat, 1994.

LACHIVER Maurice, Dictionnaire du monde rural. Les mots du passé, Paris, Fayard, 1997.

MANE Perrine, « Le travail de la vigne et du vin en pays méditerranéen au Moyen Âge » dans Le vin au Moyen Âge dans le bassin méditerranéen, Paris, Office international de la Vigne et du vin, 1993, p. 19-38.

Ministère de l'Agriculture, Direction de l'Agriculture, Statistique agricole de la France. Résultats généraux de l'enquête de 1929, Paris, Imprimerie nationale, 1936.

MOTTE Claude, PÉLISSIER Jean-Pierre, « La binette, l'aiguille et le plumeau : les mondes du travail féminin » dans DUPÂQUIER Jacques et KESSLER Denis (dir.), La société française au XIX ${ }^{e}$ siècle. Tradition, transition, transformations, Paris, Fayard, 1992, p. 237-342. 
NIESS Alexandre, "Champagne rouge, champagne sang. De l'épineuse question de la définition du vigneron champenois (1908-1914)» dans LACOMBRADE Philippe et Nicolas Fabien, (dir.) Vin et République 1907-2007, Paris, Pepper-L'Harmattan, 2009, p. $97-111$.

PECH Rémy, "Quelques réflexions à propos de la taille de la vigne en Languedoc méditerranéen aux $\mathrm{XIX}^{\mathrm{e}}$ et $\mathrm{XX} \mathrm{X}^{\mathrm{e}}$ siècles ", Bulletin du centre Pierre Léon d'histoire économique et sociale, $\mathrm{n}^{\circ} 2-3,1983$, p. 75-91.

PETIT Jean-Marie, Le lexique viticole $d u$ Biterrois, thèse doctorat $3^{\text {ème }}$ cycle, Faculté des Lettres et Sciences humaines, Université de Montpellier, 3 volumes, 1970.

VIAUT Alain, Flor de vinha. En Médoc paroles d'oc du vignoble à Saint-Estèphe, Bordeaux, Maison des Sciences de l'Homme de l'A quitaine, 1992.

VIGOUROUX Jean, Essai sur le fonctionnement économique de quelques très grandes exploitations dans la Camargue et le Bas-Languedoc, thèse doctorat Sciences politiques et économiques, Université de Montpellier, 1906. 


\title{
Évariste Galois et son premier article dans les Annales de Mathématiques de Gergonne
}

\author{
Jean-Michel FAIDIT \\ Historien des Sciences \\ Extrait de : Xavier LAFON, Les langues savantes, Paris, \\ Édition électronique du CTHS (Actes des congrès des sociétés historiques et scientifiques), 2017. \\ Cet article a été validé par le comité de lecture des Éditions du CTHS dans le cadre de la publication \\ des actes du $139^{\mathrm{e}}$ Congrès national des sociétés historiques et scientifiques tenu à Nîmes en 2014.
}

En 1810, Joseph-Diez Gergonne lançait ses Annales de Mathématiques ${ }^{1}$, éditées à Nîmes durant une vingtaine d'années jusqu'en 1832, relayées en 1836 par le Journal de Mathématiques pures et appliquées de Liouville. C'est dans ce premier journal de mathématiques qu'est publié le premier article d'Évariste Galois, mathématicien prodige tué à vingt ans dans un obscur duel. Étonnant parallèle, sa courte vie (1811-1832) correspond à la période d'édition de ces Annales de Gergonne. Figure majeure de l'histoire des mathématiques, Évariste Galois est l'auteur d'une "théorie de l'ambiguïté » où se profilent les idées de groupe et d'invariant qui allaient unifier l'algèbre et la géométrie, et jouer un rôle fondamental dans toutes les mathématiques, en physique classique et quantique et jusqu'en chimie. Sa disparition précoce contribue au mythe du mathématicien prodige.

\section{Études et æuvre mathématique de Galois (1811-1832)}

Né le 25 octobre 1811 à Bourg-la-Reine, il étudie à Louis-le-Grand, où il entre en 1823 en classe de Quatrième. Durant l'année scolaire 1827-1828, en classe de première, il découvre les mathématiques. En 1828, lauréat du Concours général de Mathématiques, il est exceptionnellement admis en Mathématiques Spéciales avant d'avoir obtenu son bac afin de pouvoir présenter le concours de Polytechnique «hors ligne», mais il essuie son premier échec. Il prépare à nouveau ce concours. Mais le suicide de son père, suite à une campagne de diffamation politique est probablement à l'origine de son second échec à Polytechnique en 1829, quinze jours plus tard. Il entre alors à l'École préparatoire à l'Agrégation (où il se classe second).

Il contracte alors un engagement décennal avec l'Université. Tour à tour, il publie pour la première fois dans les Annales de Mathématiques de Gergonne (éditées à Nîmes en mars 1829), tente le Prix de l'Académie en 1830, puis obtient sa Licence et ouvre un cours privé de Mathématiques. En 1831, il publie un article intéressant dans la Gazette des Écoles, dans lequel il livre sa vision de l'enseignement des Sciences.

Hormis la publication de deux articles dans le Bulletin de Férussac en juin 1830, son grand Mémoire sur les conditions de résolubilité des équations par radicaux connaît une destinée mouvementée. D'abord adressé en 1829 à Cauchy, il est révisé et soumis à Fourier en février 1830 pour le Grand Prix de Mathématique de l'Académie des Sciences. Réécrit à la demande de Poisson qui le refuse en juillet, il est égaré après la mort de Fourier, puis retrouvé en 1843 par Liouville dans les archives de Galois, qui le présente enfin à l'Académie et le publie en 1846 dans son Journal de mathématiques, lui ouvrant un rayonnement international. 


\section{Engagement républicain de Galois}

L'idéal républicain de Galois constitue un autre aspect de sa personnalité, qui va perturber son investissement en mathématiques et contribuer à abréger son existence ${ }^{2}$. En 1830, après les Trois Glorieuses, dont il est tenu à distance comme les autres élèves par le Directeur de l'École Préparatoire (à la différence des élèves de Polytechnique), il s'inscrit à la Société des Amis du Peuple de Raspail le 10 novembre et s'enrôle dans la Garde Nationale, à nouveau dissoute. Son exclusion de l'École Préparatoire le 9 décembre déclenche une polémique. Le 9 mai 1831, lors d'un banquet républicain, il porte un toast "À Louis Philippe... » avec un couteau à la main, ce qui lui vaut un premier séjour d'un mois de prison, entre le 10 mai et le 15 juin. Il est arrêté à nouveau le 14 juillet suivant, lors de la commémoration républicaine et non autorisée de la Fête de la Fédération, qui n'était pas encore fête nationale, cette fois pour port d'armes illégal et usurpation de l'uniforme de l'artillerie. Condamné le 23 octobre à six mois de prison, le duel fatal lié apparemment à une querelle amoureuse, survient lors d'un aménagement de sa peine pour raison de santé. L'hypothèse d'une provocation de la police secrète pour éliminer cet antimonarchiste n'étant pas écartée. Libéré le 29 avril, il rédige le 29 mai sa fameuse lettre «testamentaire » en maths, la veille du duel qui entraîne son décès le 31 mai $1832^{3}$.

\section{Joseph-Diez Gergonne (1771-1859)}

Joseph Diez Gergonne est né à Nancy le 19 juin 1771. Il fait ses études dans cette ville, et manifeste très jeune une inclination pour les Mathématiques. Enrôlé volontaire pour défendre la République, il participe à la bataille de Valmy (1792), puis à la campagne d'Espagne (1794). Entre-temps, il se classe premier à un oral de mathématiques de l'École d'artillerie de Chalons. Avec l'armée des Pyrénées, il participe aux sièges de PortVendres et de Collioure. La guerre avec l'Espagne ayant cessé, en 1796, son corps d'Artillerie est rappelé à Nîmes. C'est l'époque où l'on organise les Écoles centrales décrétées dans les derniers jours de la Convention, prévoyant une école pour 300000 habitants. Nîmes et le Gard étant concernés, Gergonne obtient par concours la Chaire de Géométrie transcendante en Mathématiques, poste qu'il conserve après leur transformation en Lycées en $1802^{4}$.

Féru d'astronomie, notamment auteur d'une étude théorique et observationnelle de la comète de 1807, il devient titulaire de la Chaire d'astronomie de la Faculté des Sciences de Montpellier en 1816. Peu après la Révolution de 1830, il est nommé Recteur de l'Académie de Montpellier. La surcharge d'activité occasionnée (quelque 44000 lettres et correspondances administratives en une quinzaine d'années) le conduit à la fin 1831 à cesser la publication des Annales. À la retraite en 1844, il décède à 88 ans $(4$ mai 1859) 5 .

\section{Les premières publications scientifiques}

Les publications généralistes se développent avec le dernier tiers du XVII ${ }^{\mathrm{e}}$ siècle. Le Journal des Savans et les Philosophical Transactions à Londres paraissent concomitamment en 1665, complétés par des publications académiques comme les Histoires et Mémoires de l'Académie des Sciences. Pour la plupart des disciplines, les premières publications spécialisées démarrent quasiment un siècle plus tard, dans le dernier quart du XVIII ${ }^{\mathrm{e}}$ siècle. Pour l'Astronomie, les Recueils pour les astronomes de Bernoulli sont édités depuis Bâle entre 1771 et 1779. Les Monatliche Correspondenz du Baron de Zach sont lancées depuis Jena en 1798. Il y a aussi les recueils annuels d'éphémérides, qui

2. Dalmas A., Évariste Galois, révolutionnaire et géomètre, Paris, Fasquelle, 1956.

3. Ehrhardt C., Évariste Galois. La fabrication d'une icône mathématique, Paris, Éditions de l'EHESS, 2011

4. Bouisson F., Notice biographique sur J.-D. Gergonne, ancien Recteur de l'Académie de Montpellier, Montpellier, Boehm, 1859

5. Otero M. H., Joseph-Diez Gergonne (1771-1859) : histoire et philosophie des sciences, Nantes, Centre François Viète d'histoire et philosophie des sciences et des techniques, 1997. 
comportent un cahier d'observations publiées en Additions, comme pour la Connaissance des Tems en France, à l'initiative de Lalande, ou les Berliner Astronomisches Jahrbuch (B.A.J.) fondées en 1776 en Allemagne. Pour les sciences physiques et naturelles, le Journal de physique, de chimie, d'histoire naturelle et des arts est lui aussi édité à partir des années 1770, connu sous le nom de Journal de La Métherie quand celui-ci assure son édition à partir de 1785. Il est accompagné sous la Révolution par le Journal des sciences utiles, par une société de gens de lettres, avec douze livraisons en quatre volumes rédigé en 1791 par l'abbé Bertholon, déjà auteur de La Nature considérée sous ses différents aspects, ou Journal d'histoire naturelle entre 1787 et 1789.

\section{Les premières Annales de Mathématiques}

Ainsi, les Mathématiques n'ont toujours pas de journal périodique au commencement du XIX ${ }^{\mathrm{e}}$ siècle. C'est durant son séjour à Nîmes que Gergonne décide en 1810, avec la collaboration de Thomas Lavernède les deux premières années, de fonder les premières Annales de Mathématiques pures et appliquées, désignées depuis Annales de Gergonne, livrées mensuellement et réunies par années universitaires. Elles constituent le premier grand journal de l'histoire des mathématiques (tant de recherches qu'à visée didactique). Le premier volume (1810-1811) est achevé en 1811. Jusqu'en 1832, une collection de 21 volumes (le $22^{\mathrm{e}}$ étant inachevé), réunit 948 articles, lettres ou mémoires, et près de 9000 pages émanant de 140 auteurs de tous niveaux (du simple élève de Collège aux membres de l'Institut). Le dernier volume renferme deux tomes au sens où l'entendait Gergonne, avec des années de parution allant de juillet à juin : bien qu'inachevé (il ne comporte que quatre articles et une note de Gergonne expliquant l'interruption de la publication), ce tome XXII (1831-1832) y apparaît séparément.

En filigrane, dans le Prospectus qui sert d'éditorial au premier numéro, on perçoit dans les intentions et les publics visés qu'une frustration est à l'origine de ce lancement depuis la lointaine province de Nîmes. L'isolement dans lequel Gergonne se sent est celui de nombre de mathématiciens en province, ou de militaires isolés, enseignant parfois les mathématiques ou des techniques y faisant appel, et qui souhaitent eux aussi enrichir la science de leurs propres avancées (résolution de problèmes, démonstrations de théorèmes, etc.)

Au fil des numéros, on rencontre les scientifiques les plus illustres de l'époque, le succès des Annales attirant les élites françaises et européennes : Ampère, Poisson, Cauchy, Abel, Chasles, Liouville, Crelle, Brianchon, Poncelet, Lamé, Dupin, Steiner, Plücker, Gergonne... et Évariste Galois, alors âgé de 17 ans, en classe préparatoire, qui signe ici son premier article (1829). Abordés sous l'angle théorique ou pédagogique, les sujets d'astronomie reviennent régulièrement au sommaire des Annales, aux côtés de ceux d'algèbre, d'analyse, d'arithmétique, de mécanique, d'optique, de physique théorique, de gnomonique et de "philosophie mathématique », domaine cher au rédacteur.

Scrupuleux jusqu'au détail pour la qualité des illustrations, notamment des figures sur des planches à part, Gergonne s'initie à la gravure. Ses quelque 200 articles reflètent la diversité de ses contributions en mathématiques, comme son principe de dualité en géométrie, (objet d'une célèbre polémique avec Poncelet) ou encore le théorème de Gergonne, le point et le triangle de Gergonne ainsi qu'une originale Arithmétique politique où il établit que des élections à partir d'assemblées représentatives peuvent aboutir au contraire de la majorité citoyenne générale. Nombre d'articles non signés lui reviennent. Son entreprise est bientôt imitée, d'abord par Crelle qui lance en 1826 depuis Berlin son Journal de Mathématiques pures et appliquées, puis par Liouville à Paris en 1834, avec son journal au même titre qu'il ouvre en 1836, souhaitant prendre le relais des Annales de Gergonne, arrêtées trois ans plus tôt. 


\section{Évariste Galois et sa première publication dans les Annales de Gergonne}

En mars 1829, le premier article d'Évariste Galois (âgé de 17 ans), dans les Annales de mathématiques de Gergonne, porte sur le développement en fractions continues des racines d'un polynôme ${ }^{6}$. La partie entière $a$ d'un réel $x$ est le premier terme du développement de $x$; le second terme $\mathrm{b}$ est la partie entière de $\mathrm{y}=1 /(\mathrm{x}-\mathrm{a})$; le troisième terme est la partie entière de 1 / $(y-b)$; et ainsi de suite... La suite d'entiers ainsi obtenue constitue le développement en fractions continues; elle est définie de manière unique.

Si le développement en fractions continues d'un réel $x$ est périodique, $x$ se trouve alors défini par un ensemble fini d'entiers. Il était connu depuis les travaux de Lagrange que le développement en fractions continues de toute solution d'une équation polynomiale du second degré est périodique. Galois prouve que la période est symétrique si et seulement si le polynôme s'écrit sous la forme $a x^{2}-b x-a$. De plus, si un polynôme à coefficients réels admet une racine réelle $x$ dont le développement en fractions continues est périodique, alors ce polynôme admet une seconde racine réelle vérifiant la même propriété.

Ce Mémoire s'inscrit dans une problématique plus générale : la recherche des solutions d'une équation polynomiale. Au commencement du XIX ${ }^{\mathrm{e}}$ siècle, des formules exactes avaient été déterminées pour exprimer les solutions d'une équation polynomiale du second, troisième ou quatrième degré en fonction des coefficients. Il restait à établir des formules générales pour des équations polynomiales de degré supérieur. Lagrange avait reformulé la question comme la résolution d'une équation polynomiale par radicaux. Il avait émis l'hypothèse que certaines équations polynomiales ne pouvaient vraisemblablement pas être résolues par radicaux. Cette suggestion était basée sur le calcul du nombre d'expressions polynomiales à $n$ variables obtenues par permutation des variables. En 1813, Cauchy s'était déjà intéressé à cette question et étudia les permutations alors appelées substitutions, travaux précurseurs de la théorie des groupes.

\section{Les continuateurs de Galois en Mathématiques}

Les travaux de Galois sont à la source de divers développements par ses successeurs en algèbre de la Théorie de Galois et du concept de Groupes. On retiendra en particulier Félix Klein et son extension en géométrie en 1872 («Les géométries sont des groupes »), Sophus Lie en 1888, avec ses Groupes de Lie en géométrie différentielle, et Henri Poincaré à la fin du XIXe, avec sa notion de structure. Nombreux sont les mathématiciens du $X^{e}$ siècle qui s'accordent sur sa vision moderne des mathématiques ${ }^{7}$.

Plus récemment, afin de rendre hommage à sa démarche généralisante, fondatrice des mathématiques modernes, Alexander Grothendieck, Lauréat de la médaille Fields en 1966, refondateur de la géométrie algébrique, a écrit en 1981 La longue marche à travers la théorie de Galois ${ }^{8}$, manuscrit de 1600 pages où il développe les pistes de recherches actuelles et futures.

Rares sont les mathématiciens conjuguant une vie aussi brève et un impact aussi grand sur leur discipline. Le cas de Galois est exceptionnel dans l'histoire des sciences, à l'image de Carnot et de son concept fondant la thermodynamique.

6. Démonstration d'un théorème sur les fractions continues, Annales de mathématiques, T. 19 (1828-1829), p. 284301 


\begin{abstract}
Résumé
En 1810, Gergonne lançait ses Annales de Mathématiques, éditées à Nîmes durant une vingtaine d'années jusqu'en 1832, relayées en 1836 par le Journal de Liouville. C'est dans ce premier journal de mathématiques qu'est publié le premier article d'Évariste Galois, mathématicien prodige qui a ouvert la voie à l'algèbre moderne, victime à vingt ans d'un obscur duel. Étonnant parallèle, sa courte vie (1811-1832) correspond à la période d'édition des Annales de Gergonne. Outre l'analyse de cette publication et l'approche biographique, cette communication analyse les réactions de la communauté mathématique au langage mathématique nouveau introduit par Galois jusqu'à la prise de conscience de son importance par Liouville, qui publie ses travaux inédits une quinzaine d'années après sa mort.
\end{abstract}

\title{
Bibliographie
}

Annales de mathématiques pures et appliquées, Nîmes, 1810-1832. 22 Tomes.

Consultables en ligne : http:// www.numdam.org/numdam-bin/feuilleter?j=AMPA

BOUISSON Fernand, Notice biographique sur J.-D. Gergonne, ancien Recteur de l'Académie de Montpellier, Montpellier, Boehm, 1859, 14 p.

OTERO Mario H., Joseph-Diez Gergonne (1771-1859): histoire et philosophie des sciences, Nantes, Centre François Viète d'histoire et philosophie des sciences et des techniques, 1997.

DaLmas André, Évariste Galois, révolutionnaire et géomètre, Paris, Fasquelle, 1956.

EHRHARDT Caroline, Évariste Galois. La fabrication d'une icône mathématique, Paris, Éditions de l'EHESS, 2011.

GALOIS Évariste, Démonstration d'un théorème sur les fractions continues, Annales de Mathématiques, $1^{\text {er }}$ mars 1829, tome 19 (1828-1829), p. 284-301.

GROTHENDIECK Alexander, La longue marche à travers la théorie de Galois, 1981, Manuscrit. (Partiellement édité sous la direction de Jean Malgoire, Montpellier, 1995, vol I, 252 p.).

Neumann Peter M., The mathematical writings of Évariste Galois (Heritage of European Mathematics), Zürich, European mathematical society, 2011. 


\section{Illustrations}

Évariste Galois à 15 ans : Artiste inconnu, extrait de Paul Dupuy, La vie d'Évariste Galois, annales scientifiques de l'École Normale supérieure, Paris, 1896.

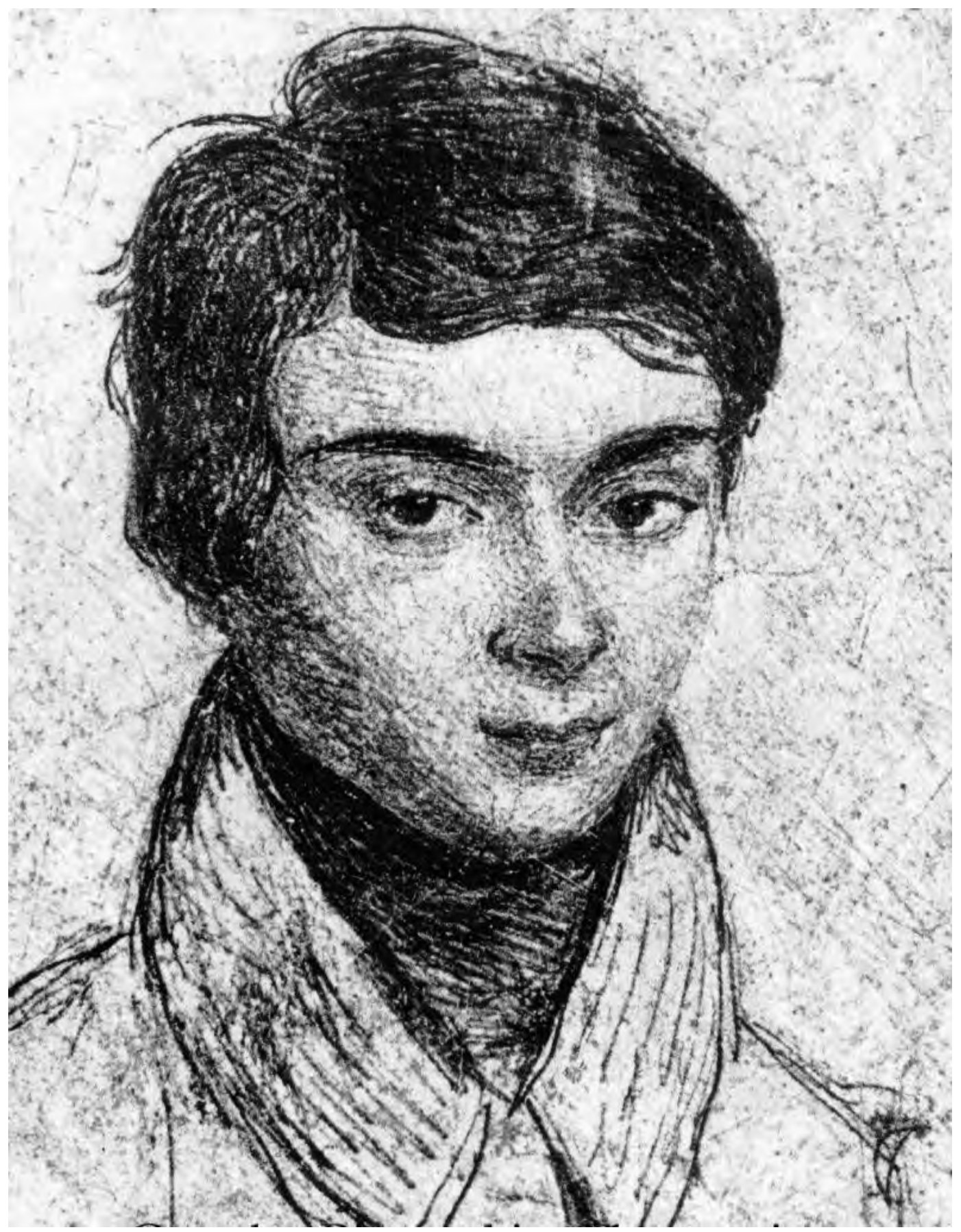


Les Annales de Mathématiques de Gergonne, contenant la première publication d'Évariste Galois en mars 1829.

\title{
ANNALES
}

$\mathrm{DJ}_{*}$

\section{MATHEMATIQUES \\ PURES ET APPLIQUEES.}

\author{
RECUEIL PÉRIODIQUE,

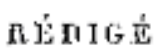

Par J. D. GERGONNE el J. E. THOMAS-LAYERNEDE.

TOHE PREMIER.

\author{
A NISHES,

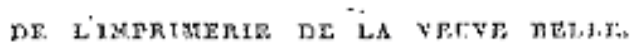

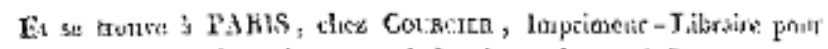

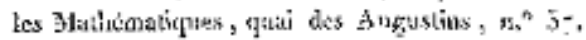

$$
\text { IEि } 51817 .
$$


Joseph-Diez Gergonne. (Cliché collection Université Montpellier II).

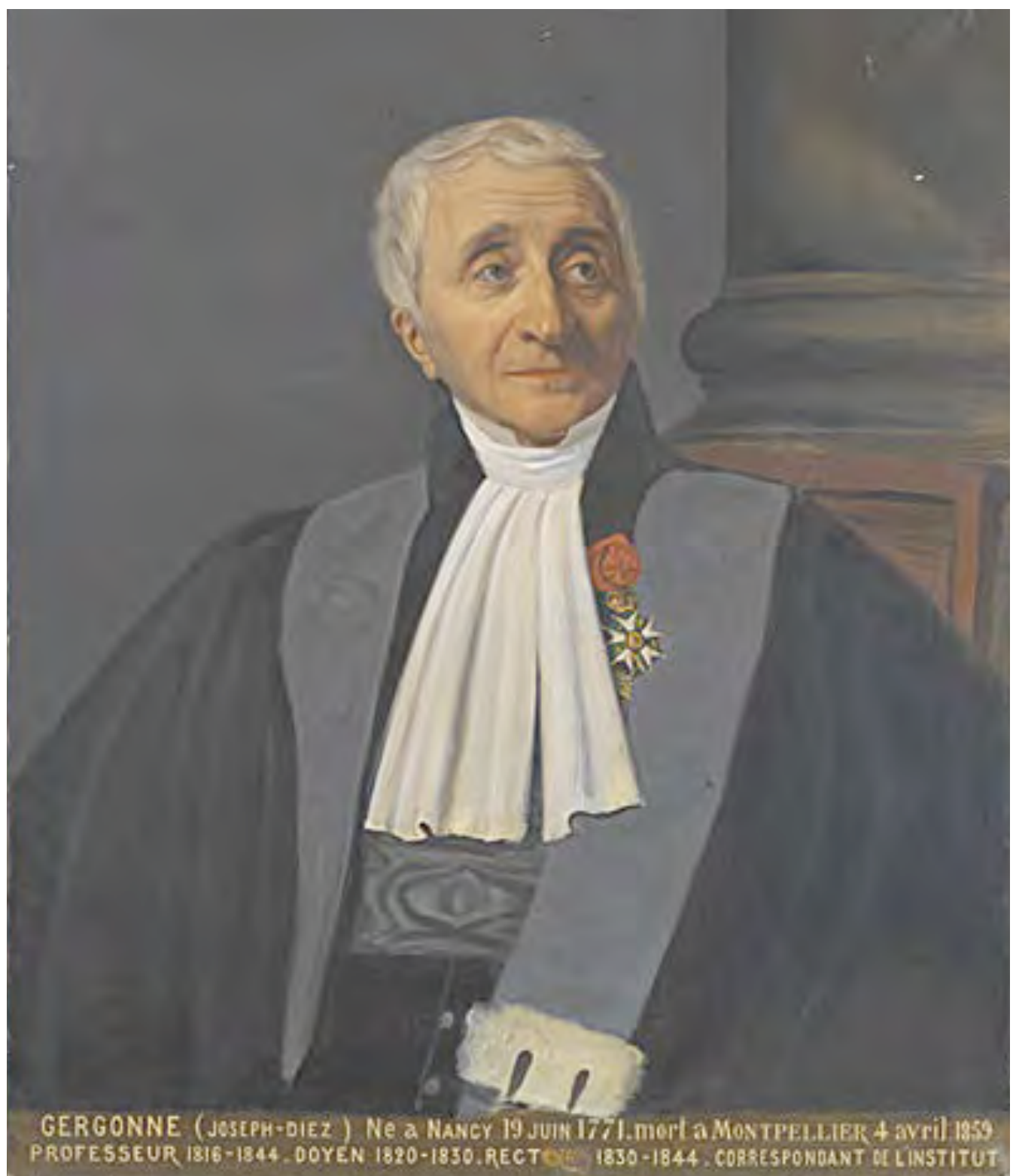


Cratère Galois sur la Lune. (Coordonnées lunaires : $14.2^{\circ}$ Sud - $151.9^{\circ}$ Ouest) (Face cachée) - Diamètre : $222 \mathrm{~km}$ - Profondeur : inconnue - Année de dénomination : 1970.

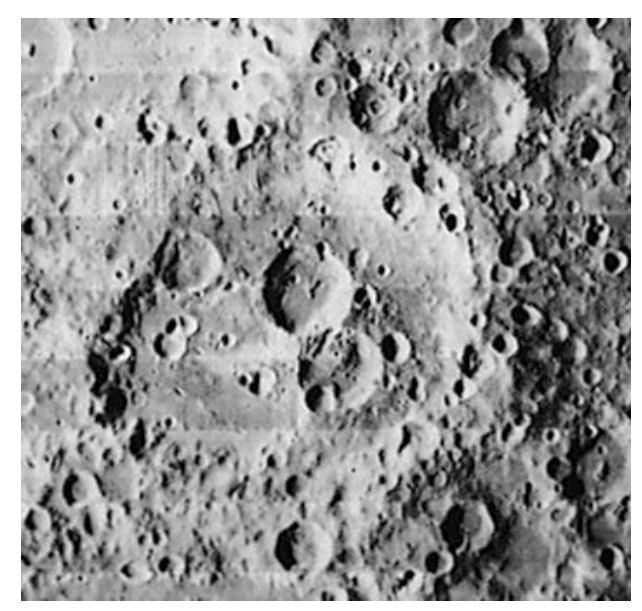

Joseph Liouville, éditeur du Journal de Mathématiques Pures et Appliquées, qui retrouva et publia les travaux de Galois en 1846, leur assurant une diffusion internationale.

(@wiki media-commons).

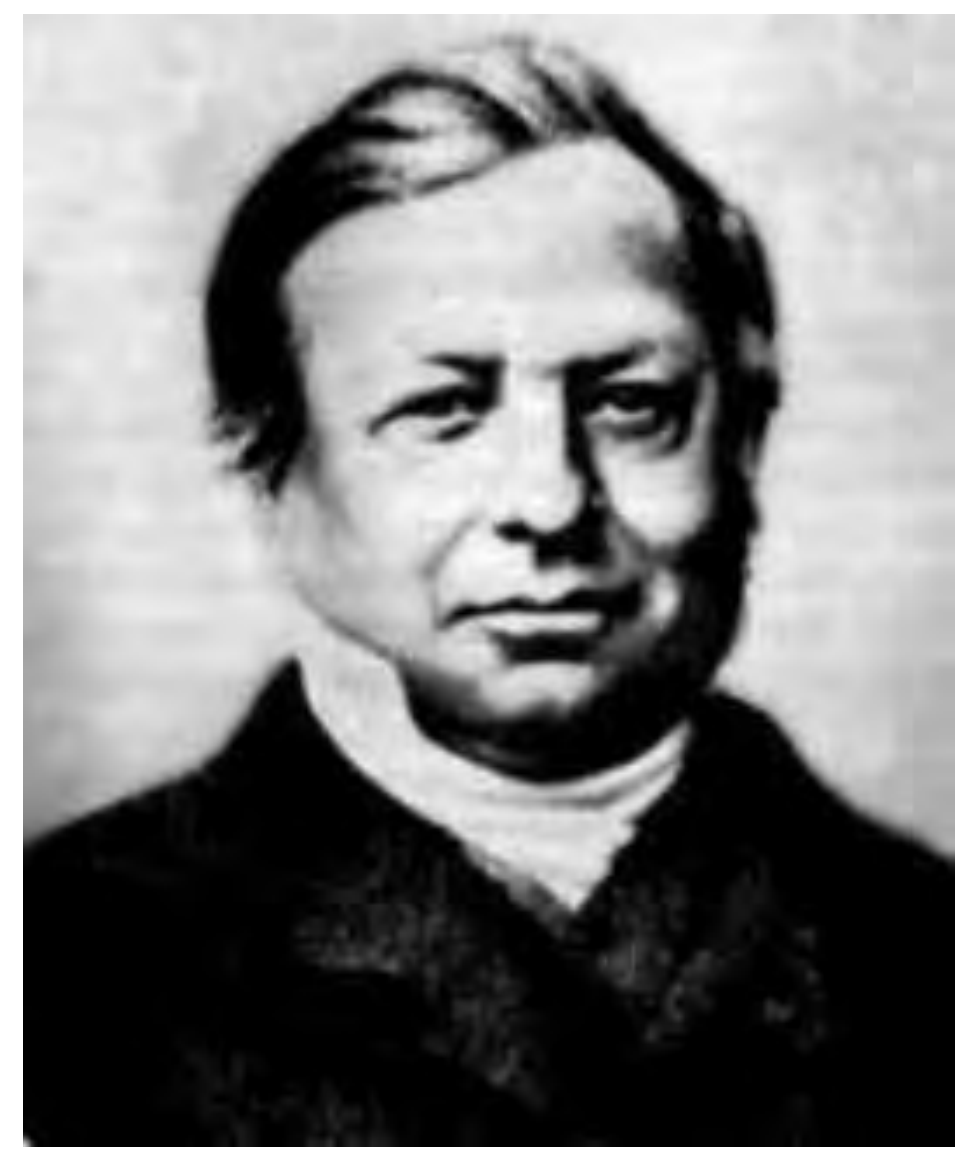


Alexander Grothendieck, auteur de La longue marche à travers la théorie de Galois en 1981. (source : http: / / www.grothendieckcircle.org).

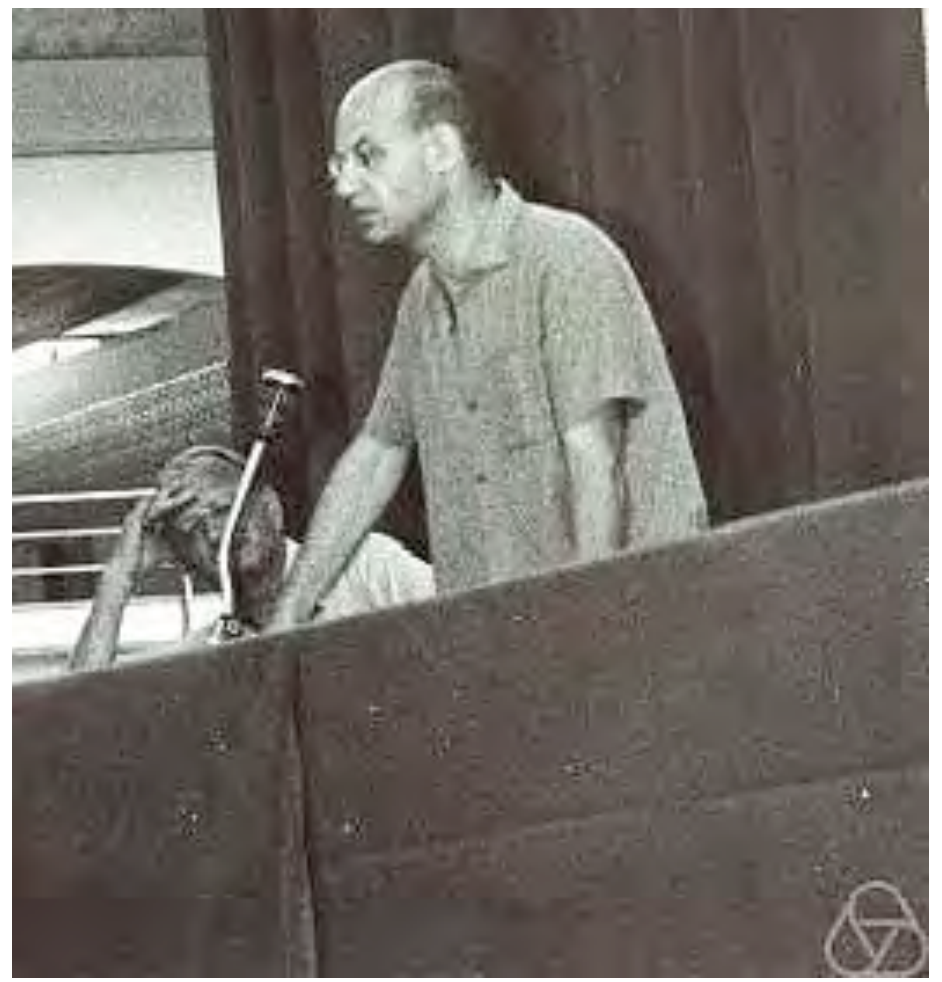

Duel au pistolet, encore très en vogue au XIX ${ }^{\mathrm{e}}$ siècle. ( $\mathrm{D}^{\prime}$ après Bauce et Rouget, 1857)

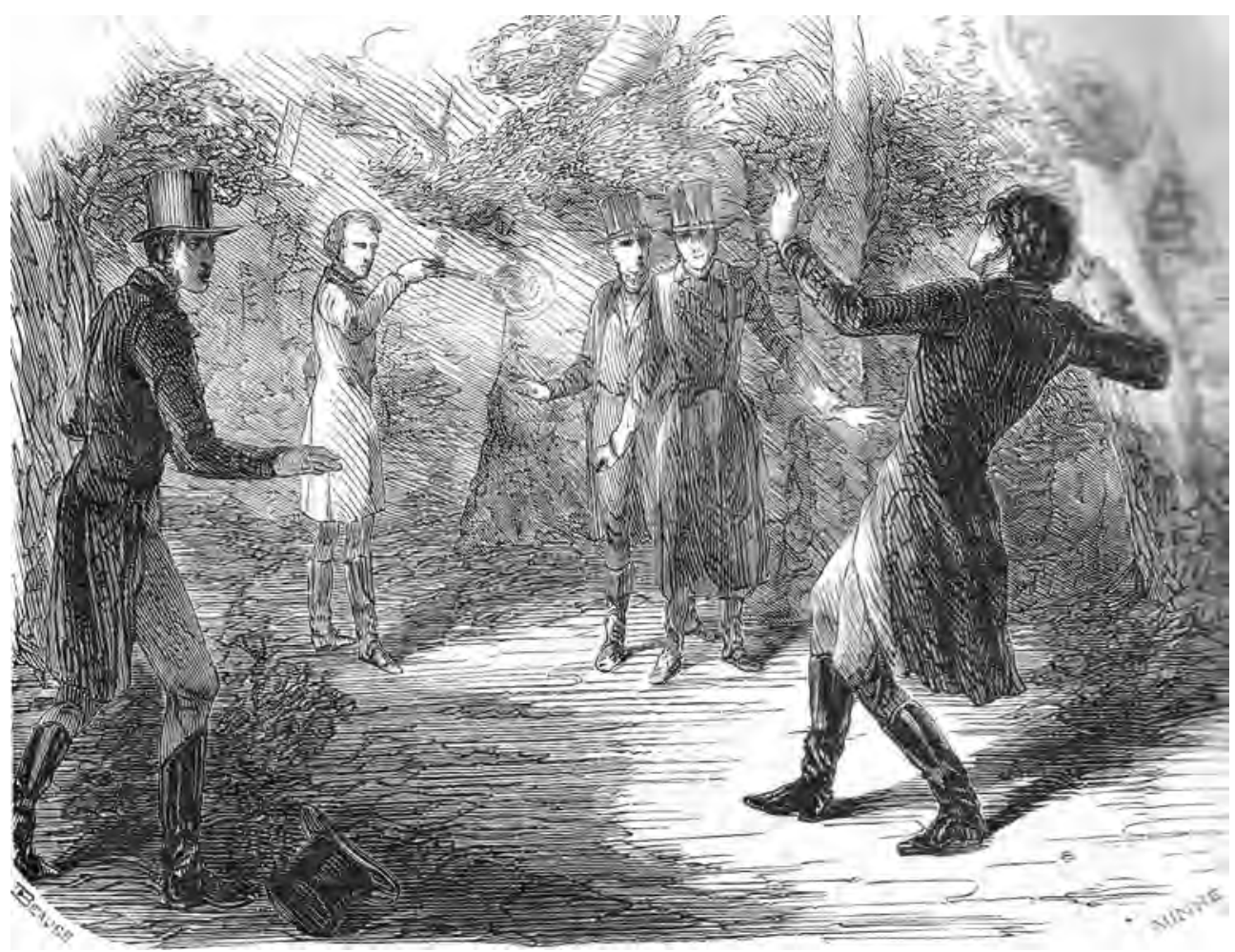


Cénotaphe de Galois à Bourg-la-Reine. (Cwiki media-commons).

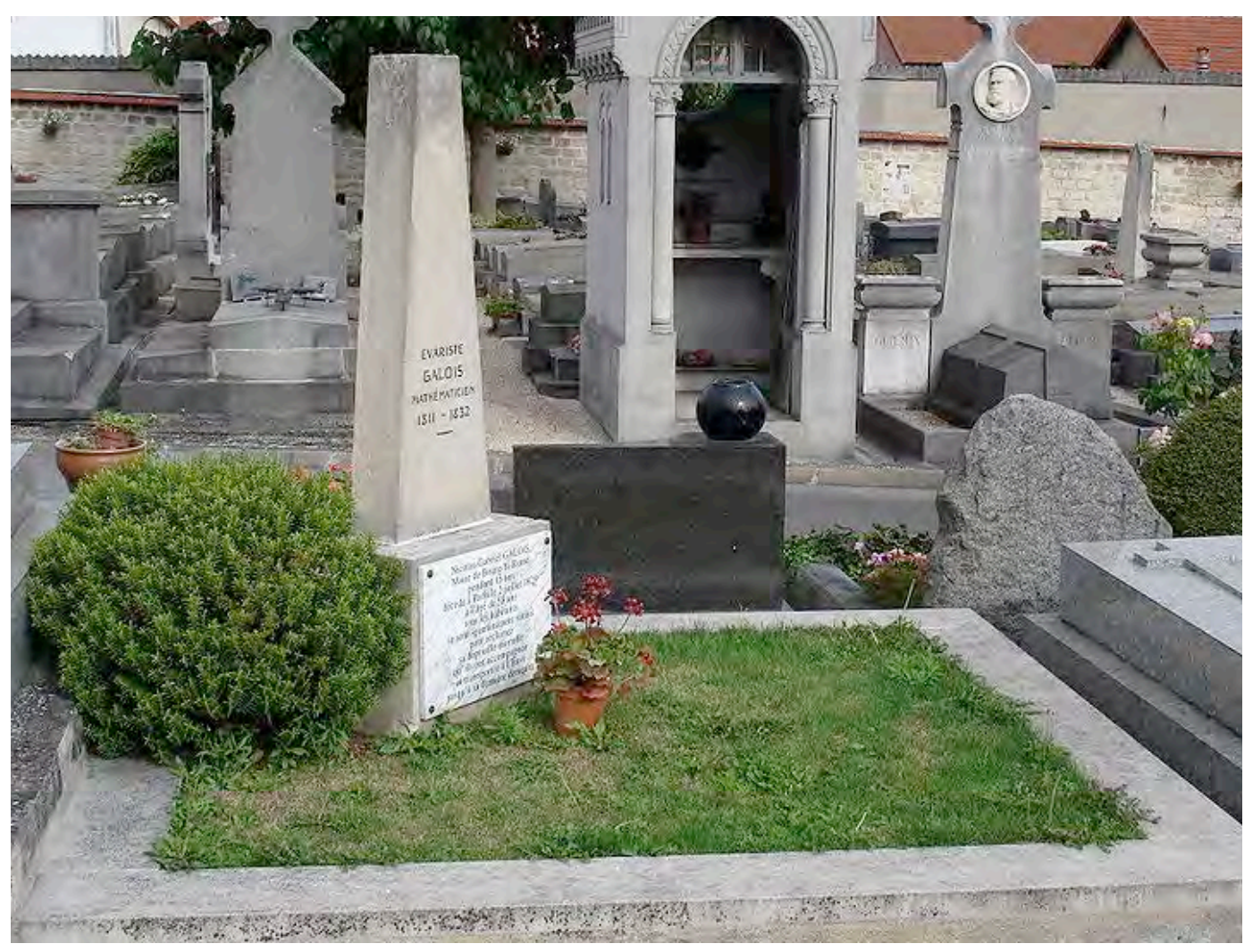




\title{
Les mots pour démolir : les instruments discursifs et les schèmes cognitifs de l'action publique sur et par l'espace
}

\author{
Philippe GENESTIER \\ Architecte-Urbaniste \\ Claudine JACQUENOD-DESFORGES \\ Linguiste \\ Université de Lyon, ENTPE, \\ CNRS UMR 5600
}

\begin{abstract}
Extrait de : Xavier LAFON, Les langues savantes, Paris, Édition électronique du CTHS (Actes des congrès des sociétés historiques et scientifiques), 2017.

Cet article a été validé par le comité de lecture des Éditions du CTHS dans le cadre de la publication des actes du $139^{\mathrm{e}}$ Congrès national des sociétés historiques et scientifiques tenu à Nîmes en 2014.
\end{abstract}

\begin{abstract}
«Parler aujourd'hui de «banlieues à problèmes » ou de " ghetto », c'est évoquer, presque automatiquement, non des «réalités » [...], mais des fantasmes, nourris d'expériences émotionnelles suscitées par des mots ou des images plus ou moins incontrôlés, comme ceux que véhiculent la presse à sensation et la propagande ou la rumeur politiques. Mais, pour rompre avec les idées reçues et le discours ordinaire [...], il faut, plus que jamais, pratiquer la pensée paradoxale qui, dressée à la fois contre le bon sens et les bons sentiments, s'expose à apparaître aux bien-pensants des deux bords soit comme un parti pris, inspiré par le désir $\mathrm{d}^{\prime}$ " épater le bourgeois », soit comme une forme d'indifférence insupportable à l'égard de la misère des plus démunis. [Il faut] rompre avec les fausses évidences, et avec les erreurs inscrites dans la pensée substantialiste des lieux [...] » ${ }^{1}$
\end{abstract}

La démolition des grands ensembles de logements sociaux : bon sens ou absurdité ? Le sens commun hésite, tiraillé entre une conception des cités HLM faisant de celles-ci des repoussoirs, des résidences indignes, pathogènes, dont la démolition serait nécessaire et salutaire, et une conception opposée qui s'inquiète, voire s'offusque de la destruction de logements abordables aux plus défavorisés, d'autant plus précieux que l'on vit une crise du logement ne cessant de s'accroître en une période où la pauvreté et la précarité progressent.

Entre ces deux perceptions, également fondées, il n'y a pourtant pas de discussion: depuis 15 ans, c'est la première qui a triomphé, et depuis 2003, la France a mis en place le Programme National de Rénovation Urbaine, prévoyant de démolir 250000 logements sociaux dans le cadre d'opérations dont le coût s'élève au total à 44 milliards d'euros. Pourquoi cette perception s'est-elle imposée au détriment de la perception opposée ?

Le PNRU est largement analysé par la sociologie urbaine et par la science politique. Ainsi sait-on tout (ou presque) sur les intérêts en jeu, sur les processus institutionnels de décision, et même sur les répercussions (voulues ou pas) des mesures prises par les pouvoirs publics en faveur (ou à l'encontre) des cités HLM.

En revanche, on sait moins de choses sur un aspect pourtant fondamental de cette politique publique : celui du cadre épistémologique et idéologique qui préside à sa conception et celui de la signification que les acteurs attribuent à leur action. En effet, la rénovation urbaine reste énigmatique, tant elle paraît à la fois paradoxale et paroxystique, d'une part, parce qu'au cours des décennies précédentes ces logements aujourd'hui détruits avaient bénéficié de plusieurs campagnes de réhabilitation (réparation, remise aux normes) et, d'autre part, parce qu'ils sont souvent localisés au sein d'agglomérations urbaines où les logements accessibles aux plus pauvres manquent cruellement. L'énigme

1. P. Bourdieu, 1993, « Effets de lieu », dans P. Bourdieu (dir.), La misère du monde, Paris, Le Seuil, p. 159. 
concerne particulièrement ce que doivent postuler et présupposer les acteurs de ces démolitions et réaménagements pour qu'une action de ce type et de cette ampleur puisse être conçue, justifiée et réalisée. Autrement dit, quelles représentations et quelles valeurs ont dû s'imposer pour qu'ait lieu cette destruction massive de logements sociaux?

Ce texte tente, par une analyse de discours relatifs à la politique nationale de démolition des grands ensembles HLM, de mettre au jour les procédés discursifs et les schèmes de pensée qui conduisent à appréhender le malaise social en termes spatiaux, au point de concevoir les actions publiques devant y porter remède, elles aussi, en termes spatiaux.

Ainsi, les «mots de l'espace $»^{2}$ servant à exprimer les malaises sociaux («exclusion », « marginalisation », « relégation »), les métaphores conceptuelles ${ }^{3}$ structurant nos façons de décrire la ville («tissu urbain», «maillage viaire»), les métonymies («quartiers », «banlieue»), les syllepses («urbanité», « espace public»), les paronomases («lieu et lien », « social et spatial », « urbain et humain »), les étymologismes (« cité », « civilité », « citoyenneté ») sont quelques-uns des instruments discursifs par le truchement desquels l'action publique fabrique ses thématiques, élabore ses problématiques et trouve des solutions plausibles aux problèmes ainsi posés.

Ces expressions spatialisantes ne sont pas, en effet, que des procédés stylistiques : elles fonctionnent également comme des concepts opératoires qui acquièrent une puissance d'action dans un contexte épistémologique particulier qui les accrédite et les renforce. Ce contexte, qui s'est imposé dans le monde des sciences sociales françaises actuel, nous pourrions le qualifier de manière schématique de "mésoscopique » (échelle de lecture du réel se situant entre l'échelle macroscopique propre au structuro-fonctionnalisme et celle, microscopique, de l'individualisme méthodologique). L'analyste qui appréhende le réel à l'échelle mésoscopique tient de facto compte de la dimension spatiale de ce dernier et considère pleinement (bien que de manière variable selon les écoles de pensée et les disciplines) le facteur environnemental. En cela, cette échelle de lecture contraste avec celles qui, hier, étaient dominantes. En effet, les échelles macroscopique et microscopique, bien qu'opposées, étaient quant à elles identiquement indifférentes à l'espace. De plus, la prise en compte de la spatialité des phénomènes sociaux par la grille actuelle d'appréhension et de compréhension (plus que d'explication) du réel sous-tend une démarche technico-politique qui attribue à l'espace physique une capacité à déterminer, soit matériellement, soit symboliquement, l'identité et le comportement de ceux qui l'habitent. Dès lors, la lecture mésoscopique fait de l'espace physique un actant. Ainsi, parle-t-on aujourd'hui d' «effet de quartier» dans les banlieues pour évoquer ce dont sont victimes certains de leurs habitants, ce qui s'exprime en termes de ségrégation et de stigmatisation. On assisterait donc actuellement à la domination, sur le plan cognitif et épistémique, d'un discours porteur de représentations spatialisantes qui se prolongent sur le plan pratique et politique par une pensée de nature spatialiste. ${ }^{4}$

Dans ce texte, nous explorons donc en deux temps la prégnance de quelques catégories de désignation et de qualification spatialisantes. Nous examinerons d'abord la manière dont ces catégories sont inscrites dans le lexique utilisé, tant pour énoncer des questions sociales que pour appliquer des schèmes d'inférence en vue de concevoir une action publique sur l'espace (action architecturale et urbanistique). Puis nous replacerons ce complexe de présupposés et de stéréotypes portés par le lexique et la rhétorique en vigueur dans la grille de lecture (la «vision du monde », l' « épistémè »... selon la façon dont on veut la nommer) qui confère sa plausibilité, sa crédibilité et son acceptabilité aux catégories de pensée régissant l'action. Autrement dit, il s'agit pour nous d'analyser comment et pourquoi l'action publique sur et dans l'espace est censée avoir un caractère socialement opératoire et efficient, au point que l'on puisse parler $\mathrm{d}^{\prime}$ «action par

2. P. Bacot et S. Rémi-Giraud, 2007.

3. G. Lakoff et M. Johnson, 1985.

4. Selon cette pensée, l'espace physique serait un inducteur social, de sorte que les pouvoirs publics pourraient façonner les représentations et/ou les pratiques des habitants par un remodelage des lieux où ils vivent. Cf. Y. Chalas, 1989. 
l'espace ». Il s'agit ainsi de lire l'enchaînement qui conduit les pouvoirs publics, à partir du moment où ils considèrent l'espace physique comme un inducteur social, à y voir également un instrument privilégié d'intervention.

Les matériaux empiriques employés seront extraits de textes (discours gouvernementaux, articles de presse généraliste et spécialisée...) collectés dans le cadre d'une recherche réalisée de 2010 à 2013 pour le compte de l'Agence Nationale de la Recherche, intitulée "La démolition dans les grands ensembles: un effet paroxystique des attentes et attendus en matière d'Espace » (dont le responsable scientifique était P. Genestier).

\section{Les instruments discursifs créant une confusion entre le spatial et le social}

Le discours de la rénovation urbaine se caractérise par des confusions constantes entre deux registres référentiels : celui du social et celui du spatial.

Sur le plan lexical, on observe que les promoteurs de la rénovation urbaine conçoivent la ville comme une entité devant rassembler une population dans son intégralité. Pour en décrire les dysfonctionnements, ils opposent en effet les termes exprimant une idée d'exclusion ("marginalité », «isolement», «relégation ») à ceux exprimant une idée d'inclusion ("réinsertion", "intégration", "accès à l'emploi / aux soins / aux services ») :

«Il s'agit de prendre conscience qu'humainement, socialement, il n'est plus possible de laisser des gens au ban de notre société. En cela, la lutte contre l'Exclusion sociale et la Pauvreté est un sujet politique majeur. Nous ressentons tous, dans la population, une forme de démobilisation généralisée, une désaffection émotionnelle vis-à-vis des plus pauvres. Ces derniers peuvent bien souffrir, un mur invisible, aussi étanche qu'une vitre d'aquarium semble les séparer du reste de la communauté humaine. Ils survivent tant bien que mal sous notre œil apathique dans le "ghetto" de l'exclusion. La séparation et la relégation prévalent. En cela, le terme même d'« exclus» me déplaît. Je le trouve lourd de conséquences, d'enfermements... $»^{5}$

Vue sous cet angle, la rénovation urbaine a pour mission la réintégration des exclus dans la ville, que ce soit dans un objectif humaniste ou politique :

«Avec la rénovation urbaine, c'est finalement la première fois dans l'histoire de la politique de la Ville que l'on aborde sérieusement la mutation physique des quartiers sensibles et la contribution qu'elle apporte au bien-être et à l'image de ses résidents. On peut rétorquer que l'on s'attache au territoire et pas assez aux gens qui l'habitent. Que cette rénovation ne serait finalement qu'un "cache-misère". Mais lorsque vous habitez une architecture qui vous indique votre place sociale de relégués, est-ce «criminel», comme certains l'ont exprimé, d'investir des millions pour permettre de vivre dans un cadre qui offre de vivre communément « en ville » et pas dans ce qui est devenu un « désigne-misère »? "

«Pour le ministre de l'Emploi, de la Cohésion sociale et du Logement [Jean-Louis Borloo], ce dossier [le financement de la rénovation urbaine] est prioritaire. Il l'a rappelé hier : « Il s'agit d'extraire le poison de la ségrégation sociale et territoriale [...] Il y a là tous les fondements du délitement républicain. Nous entrerons en 2007 avec le sentiment que notre pays est en train de le traiter. »

C'est pourquoi les opérations de rénovation urbaine sont si souvent décrites comme des actions de «désenclavement» de quartiers jugés trop isolés du reste de la ville et,

5. Christine Boutin, Ministre du Logement et de la Ville, Réunion plénière du conseil national de lutte contre la pauvreté et l'exclusion sociale, 21 juin 2007.

6. Frédéric Winter, «Chroniques de la rénovation urbaine», Urbanisme, septembre 2010

7. «Davantage d'argent pour réhabiliter les banlieues », Le Figaro, 23 mai 2006. 
particulièrement, du centre-ville. Ainsi peut-on lire dans des prospectus municipaux pour des projets de réaménagement à Vaulx-en-Velin :

«La réalisation de ces projets accentuera le dynamisme du centre-ville et renforcera par ailleurs son rôle fédérateur grâce à de nouvelles liaisons et à une continuité urbaine vers plusieurs quartiers de la ville. ${ }^{8}$

Le réaménagement de l'avenue Salvador Allende se poursuit (...) L'objectif est de donner à cette avenue un profil plus citadin et de faciliter les liaisons avec les quartiers environnants. ${ }^{\overline{9}}$

À cet égard, la figure métaphorique ${ }^{10}$ du tissu est partout présente dans le discours de la rénovation urbaine pour évoquer l'idée du lien. Il s'agit d'une métaphore conceptuelle ${ }^{11}$ structurant notre conception de la ville et, plus largement, des territoires urbanisés. Dans cet univers sémantique, on rencontre des expressions comme " tissu urbain », " maillage viaire " ou "franges urbaines», ainsi que le substantif «trame», notamment dans des syntagmes plus ou moins figés comme "trame viaire», "trame verte» ou "trame bleue ». De même, l'on parlera par exemple de «recoudre l'urbain», de «remailler le quartier », de «tisser des liens » entre les différentes parties d'une ville :

« Le SCOT [schéma de cohérence territoriale] identifie les quartiers Est [...] comme des lieux privilégiés de renouvellement urbain [...] et fixe comme orientation sur ces territoires [...] un élargissement du processus de renouvellement urbain permettant de mieux relier ces quartiers au tissu urbain et d'affirmer leur capacité à conjuguer qualité et intensité

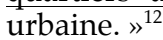

«Sur les quartiers Est, trois objectifs majeurs [...] sont à réaffirmer : organiser les continuités piétonnes afin de permettre les déplacements dans le cœur des quartiers et vers les autres territoires de la ville, construire un maillage de voies afin de désenclaver les quartiers et les ouvrir vers l'extérieur, et enfin améliorer les espaces extérieurs pour une meilleure qualité de vie. ${ }^{13}$

L'analogie sous-jacente entre une ville (le thème) et un ensemble de fils qui s'entrecroisent (le phore) est fondée sur l'idée que les différentes parties d'une ville doivent être reliées les unes aux autres, autrement dit, idéalement, une ville doit constituer un ensemble cohésif ${ }^{14}$. L'idée qui sous-tend ce postulat aménagiste est que relier les quartiers les uns aux autres ne peut que faciliter les relations entre leurs habitants.

C'est pourquoi il est si souvent question de «recomposer», « restructurer», «redistribuer», «reconfigurer» $l^{\prime}$ « urbain» ou $l^{\prime}$ « espace public» de manière à le « réinsérer» dans « la trame viaire environnante » ou «le fonctionnement d'ensemble de la ville ».

L'emploi généralisé d'autres figures de rhétorique telles que les métonymies ${ }^{15}$, synecdoques ${ }^{16}$, syllepses ${ }^{17}$ et zeugmes ${ }^{18}$ contribue à amalgamer le social et le spatial.

8. Dépliant « Le cœur de ville grandit », ANRU, Grand Lyon, Vaulx-en-Velin, mars 2010.

9. Dépliant «Les projets par quartiers, Vernay-Verchères, La rénovation urbaine du quartier », ANRU, Grand Lyon, Vaulx-en-Velin, juillet 2013.

10. Métaphore : figure sémantique reposant sur un rapport d'analogie entre l'objet désigné par le sens propre (thème) et l'objet désigné par le sens figuré (phore).

11. G. Lakoff et M. Johnson, op. cit.

12. Dépliant « Vaulx-en-Velin, Centre Ville / Quartiers Est », Assemblée générale du quartier du 10 juin 2010.

13. Dépliant «Vaulx-en-Velin, Centre Ville / Quartiers Est », op. cit.

14. Ce type de métaphore peut également contribuer à produire des effets spatialisants, notamment avec l'expression «tissu social » très usitée dans notre corpus, pour peu qu'elle s'insère dans un zeugme, comme dans ce titre d'un article de La Croix, paru le 13 mai 2009: "A la pelleteuse, la ville de Châteauroux reprise son tissu social ».

15. Métonymie : figure sémantique reposant sur un rapport de contiguïté entre l'objet désigné par le sens propre et l'objet désigné par le sens figuré.

16. Synecdoque : figure sémantique reposant sur un rapport d'inclusion entre l'objet désigné par le sens propre et l'objet désigné par le sens figuré. (Nous considérons la synecdoque comme un cas particulier de métonymie.) 
Ces figures opèrent en effet une confusion entre, d'une part, les thématiques spatiales portant sur des lieux urbains jugés défectueux et, d'autre part, les thématiques sociales, à savoir le mode de vie de leurs habitants, en sous-entendant que ce mode de vie est influencé par les caractéristiques de leur lieu de résidence.

$1^{\text {er }}$ exemple :

« Il ne s'agit pas de faire de la réparation, il s'agit de casser les ghettos, c'est-à-dire [...] de recomposer dans ces quartiers une vie normale de ville, dans un esprit de mixité sociale... $»^{19}$

Dans ce contexte, la syllepse du ghetto peut référer simultanément à un quartier isolé qu'il s'agit de réintégrer - au sens propre - dans une ville par un acte concret de démolition ( $\mathrm{d}^{\prime}$ où l'emploi du verbe «casser » ${ }^{20}$ ) et à une communauté isolée qu'il s'agit de réintégrer - métaphoriquement - dans une population plus vaste. Autrement dit, pour Marie-Noëlle Lienemann, il semble que favoriser l'émergence d'une "vie normale » en ville suppose des actions de réaménagement urbain visant à désenclaver des quartiers jugés trop isolés.

$2^{\mathrm{e}}$ exemple :

«L'objectif de ce programme est clair : la transformation profonde des quartiers les plus en difficulté de notre pays... $\gg^{21}$

Le syntagme nominal «transformation profonde des quartiers les plus en difficulté » correspond à un zeugme ${ }^{22}$ associant le sens propre du terme «quartiers» («partie de ville en tant que lieu») à son sens figuré («habitants vivant dans cette partie de ville»). En effet, dans un contexte urbanistique, on parle généralement de "transformation » pour désigner des actions portant sur des lieux, alors que la locution adjectivale « en difficulté " qualifie plutôt des situations humaines. Si Claude Bartolone avait voulu aborder une thématique spécifiquement urbanistique, il aurait parlé des «quartiers les plus dégradés», par exemple, car l'adjectif «dégradé » peut qualifier des objets architecturaux ou urbanistiques selon l'usage ordinaire de la langue française. Le zeugme crée ici implicitement une confusion entre action exercée sur le quartier en tant que lieu et action exercée sur la population habitant ce quartier.

Dans le discours de la rénovation urbaine, le mot « quartiers » (au pluriel) constitue sans doute l'une des métonymies les plus courantes. Ce mot est en effet fréquemment associé à des qualificatifs dont l'univers sémantique relève de l'humain et/ou du social, tels que : « difficiles », « sensibles », « fragiles », « vulnérables », « malades », « déshérités », « défavorisés ». Cela signifie entre autres que les problèmes sociaux sont appréhendés au travers des lieux plutôt qu'au travers des personnes.

Ce type de métonymies nous amène à percevoir les habitants des quartiers de grands ensembles comme des populations spécifiques et homogènes, dont tous les membres

17. Syllepse : figure sémantique reposant sur un rapport de simultanéité entre un sens propre et un sens figuré.

18. Zeugme : figure syntaxique associant des éléments hétérogènes (concrets et abstraits par exemple) au sein d'une même phrase.

19. Marie-Noëlle Lienemann, secrétaire d'État au logement, Congrès UNSFA - Marseille, 5 octobre 2001.

20. L'expression «casser les ghettos" est une formule forgée par Marie-Noëlle Lienemann pour qui, typiquement, la transformation du social s'effectue par une action sur l'espace, ainsi qu'on peut l'observer dans ses discours par la présence d'autres expressions de structure similaire, comme "bâtir une civilisation " (Congrès UNFSEA, Marseille, 5 octobre 2001), où un verbe exprimant une action urbanistique est associé à un concept comportant une dimension sociale.

21. Claude Bartolone, ministre délégué à la Ville, Démolition de la tour 5, Tarterêts, Corbeil-Essonnes, le 3 avril 2000.

22. Ce zeugme est structuré de la façon suivante : nom désignant une action urbanistique + nom désignant un objet urbanistique et/ou social sur lequel porte l'action urbanistique + adjectif désignant une propriété sociale de l'objet sur lequel porte l'action urbanistique. 
partageraient les mêmes caractéristiques sociales et, par là même, les mêmes besoins, du seul fait qu'ils résident au sein du même environnement urbain ${ }^{23}$. Réaménager l'ensemble d'un quartier est donc censé résoudre indifféremment les problèmes sociaux de tous ses habitants. Selon P. Genestier, « la pensée métonymique, par subsomption, stipule que le partage d'une même localisation constitue un facteur unificateur et identificateur » des populations qui y vivent. D'autre part, précise-t-il, "les figures métonymiques "ville", « cité », « quartier » procèdent par hypostase car elles saisissent le réel au travers de ses manifestations spatiales, c'est-à-dire tangibles, visibles et lisibles, voire médiatiquement représentables, tout en postulant la force d'homogénéisation du lieu de résidence ${ }^{24}$.

Ces confusions entre le social et le spatial sont parfois renforcées par des paronomases qui, en rapprochant des termes qui se ressemblent sur le plan phonétique, donnent faussement l'impression qu'ils sont unis sémantiquement (ex.: «lieu» et «lien», « social » et « spatial », « humain et urbain ») :

«Pour nous tous, ce lieu est un facteur essentiel du lien social dans nos rues. $»^{25}$

De même, il est fréquent que des jeux de mots fondés sur des étymologismes mettent en relation des objets spatiaux et des groupes sociaux, comme "les cités" au sens d'agglomérations d'immeubles et "la cité » au sens de communauté politique, et avec d'autres termes d'origine commune comme «civilisation», «citoyenneté » ou « civilité ${ }^{26}$ :

« Notre civilisation est bel et bien devenue celle de la cité. ${ }^{27}$

Sur le plan du raisonnement argumentatif, des confusions entre le social et le spatial apparaissent dans la façon dont les promoteurs de la rénovation urbaine présentent leurs arguments, laissant entrevoir le caractère illusoire des inférences ${ }^{28}$ qui sous-tendent leurs raisonnements.

Examinons à cet égard le discours de Gérard Bonhomet, adjoint à l'urbanisme et à l'habitat à Garges-lès-Gonesse. Pour cet élu, il est évident que les grands ensembles génèrent de l'insécurité, contrairement aux "petits immeubles résidentialisés », et c'est pourquoi il se félicite de leur démolition :

« Débuté en 2005 avec la démolition des premiers logements, pour arriver à 539 à ce jour sur les 998 existant au départ, le programme a déjà singulièrement changé la physionomie du quartier, «il y a cinq ans encore véritable zone de non-droit, mais où aujourd'hui les gens, relogés dans de petits immeubles résidentialisés tout neufs de trois ou quatre étages maximum, ont retrouvé la sécurité et le plaisir d'échanger » précise l'élu. ${ }^{29}$

En nous inspirant du modèle de S. Toulmin $(1958)^{30}$, nous analysons l'argumentation de cet élu de la manière suivante :

23. D'après G. Lakoff et M. Johnson (1985, p. 45), la figure métonymique « nous permet de nous concentrer plus spécifiquement sur certains aspects de l'entité à laquelle nous nous référons ». Ici, c'est l'aspect " habiter un quartier dégradé » qui est sélectionné pour désigner des groupes de personnes.

24. Cf. http://www-ohp.univ-paris1.fr/Textes/Genestier_2.pdf.

25. «Le Smoke ne partira pas en fumée », L'Humanité, 26 octobre 2007.

26. L'étymologisme consiste à utiliser "une racine étymologique commune comme raison suffisante pour établir une parenté sémantique et/ou référentielle entre divers termes. Ainsi, la racine cit permet de gommer la distinction entre l'urbs [espace physique de la ville] et la civitas [communauté politique], entre l'astu [ville vue de l'intérieur, dans sa quotidienneté] et la polis [ville vue de l'extérieur comme entité politique]. La référence explicite à l'origine latine civis des mots comme " cité ", " citoyenneté ", " civilité », " civilisation » participe du cadrage urbain des questions sociales », ainsi que l'écrit P. Genestier (2010, p. 254).

27. «2007. Ce qui doit changer. Dossier. Des villes plus conviviales », La Croix, 13 mars 2007.

28. M. Dufour, 2008.

29. Interview de Gérard Bonhomet, «Garges-lès-Gonesse. La rénovation de la Muette attire de nouveaux habitants », Le Parisien, 8 août 2009.

30. Selon ce modèle, un discours argumentatif pleinement développé se structure en cinq composantes fonctionnelles dont l'articulation constitue une «cellule argumentative »: la prémisse, la loi d'inférence, le support, la réserve et la conclusion. Ces composantes sont reliées entre elles de la façon suivante : la conclusion, 
- prémisse 1: l'insécurité régnait dans le grand ensemble dont la démolition a été entreprise ;

- prémisse 2: l'insécurité ne règne pas dans les petits immeubles résidentialisés qui remplacent les logements démolis du grand ensemble ;

- loi d'inférence : le grand ensemble est générateur d'insécurité ;

- conclusion : la rénovation urbaine permet de résoudre le problème de l'insécurité en remplaçant les grands ensembles par de petits immeubles résidentialisés.

Cette argumentation comporte ce que nous appelons un «saut inférentiel » fondé sur une confusion de causalités, plus précisément, sur une confusion entre les effets des caractéristiques architecturales de l'habitat et ceux, probablement plus prégnants, des caractéristiques psychosociales des habitants.

Nombre d'argumentations de ce type renvoient en fait à des raisonnements abductifs, où une simple corrélation vaut pour une relation de cause à effet :

- prémisse $1:$ l'insécurité règne dans tel ou tel quartier ;

- prémisse 2 : ce quartier comporte des grands ensembles ;

- conclusion : cette insécurité est due à la présence de grands ensembles.

Plus spécifiquement, le discours de la rénovation urbaine met en avant des notions qui sont présentées comme des valeurs morales, censées fédérer l'ensemble des habitants, telles que l' « urbanité », le "vivre ensemble » et le « civisme ». Ces valeurs se rapportent à des normes comportementales, à un "savoir être " social et sont constitutives du mode de vie idéal prôné par la puissance publique en France aujourd'hui. Elles se reflètent dans l'image du cadre urbain décrit par les discours de la rénovation urbaine comme étant propice à l'épanouissement de ce mode de vie : démolition des barres et des tours (jugées " pathogènes »), reconstruction de petits immeubles "résidentialisés », réaménagement de rues pour mieux circuler et de places pour mieux se rencontrer...

Les argumentations déployées par le discours de la rénovation urbaine sont généralement fondées sur des inférences fallacieuses ou, du moins, illusoires, reposant sur des confusions entre bénéficiaires de l'action de rénovation urbaine, entre causalités expliquant une situation défectueuse et, conséquemment, entre catégories d'objets sur lesquels porte l'action correctrice, entre univers de référence constituant le cadre de l'action, entre aspirations attribuées aux destinataires de l'action et aspirations des promoteurs de cette action.

Ces amalgames sont tellement ancrés dans les esprits que les discours des promoteurs de la rénovation urbaine en deviennent parfois même incohérents :

«Le constat sur le quartier des Vernay-Verchères est catégorique et partagé par tous les partenaires : son enclavement entre la rue Maurice Audin et les avenues Paul-Marcellin et Salvador Allende empêche son ouverture vers le Centre-Ville et le Village, tandis que sa vie en cœur de quartier est entravée par des impasses, une circulation difficile et des passages collectifs sous les bâtiments. Le quartier est, de plus, fragilisé par des situations sociales alarmantes : $40 \%$ de chômage chez les 16-24 ans, $33 \%$ de familles monoparentales...

Il devenait donc urgent que les habitants des Vernay-Verchères se rendent compte que leur quartier [comme d'autres de Vaulx-en-Velin] allait aussi se transformer grâce à trois orientations majeures : ouvrir le quartier sur la ville, améliorer le cadre de vie des habitants et améliorer les circulations dans le cœur du quartier.

qui peut être nuancée par une réserve, est affirmée sur la base d'une prémisse par l'application d'une loi d'inférence qui, elle-même, s'appuie sur un support. Dans la pratique, la structure des discours argumentatifs, potentiellement régressive, peut être plus complexe: elle peut comporter plusieurs prémisses justifiant une conclusion, plusieurs conclusions justifiées par une prémisse, des sous-prémisses justifiant une prémisse principale, des sous-conclusions justifiées par une conclusion principale... Elle peut être au contraire plus simple et se composer uniquement d'un «noyau » constitué de la prémisse et de la conclusion reliées entre elles par la loi d'inférence. 
Si nous savons, malheureusement, que ce projet urbain ne résoudra pas les situations sociales et économiques de certains habitants, nous sommes convaincus qu'il sera la première étape vers une amélioration de leurs conditions de vie. ${ }^{31}$

C'est ainsi qu'à Vaulx-en-Velin, une promesse de réaménagement d'un quartier est énoncée en réponse à l'évocation des problèmes sociaux qui y règnent.

Sur un autre volet du dépliant est indiqué le «coût total» de ce projet d'aménagement : « $8036000 € \mathrm{TTC}$ ». Dès lors, on peut s'interroger sur la nature du message envoyé aux habitants dans ce prospectus: il s'agit probablement de leur transmettre l'idée que les pouvoirs publics font des efforts importants pour améliorer le cadre bâti de leur vie quotidienne, le revers de la médaille étant que ces habitants pourraient s'offusquer du fait que de si grosses dépenses soient réalisées dans ce seul but.

Force est de reconnaître que modifier une forme urbaine est à la fois plus facile, plus immédiat et politiquement plus visible que chercher à améliorer la condition sociale des habitants des grands ensembles, ce qui reviendrait à vouloir lutter contre la pauvreté, la crise économique, le chômage, les déficiences éducatives et, plus problématiquement encore sur le plan politique, à se poser des questions d'ordre ethnique, etc.

Notre hypothèse est que l'engouement des pouvoirs publics pour une rhétorique de la «normalisation urbaine » constitue une réponse à la panique morale engendrée par la montée de leur impuissance face à l'ampleur des problèmes sociaux (tels que la précarité économique et les flambées de violence dans les banlieues) à laquelle ils sont confrontés. Ainsi, la spatialité serait adoptée et promue en tant que thématique de gestion du social en raison d'enjeux médiatiques et politiques, notamment parce que sa mise en exergue autorise à articuler dans un récit éthico-politique des principes généraux et des mesures réalisables, car tangibles et même visibles dès lors qu'elles sont inscrites dans l'espace physique. Or, une telle articulation est devenue aujourd'hui difficilement concevable dans bien d'autres domaines, dans celui de l'économie en particulier, où les objectifs annoncés paraissent souvent incantatoires.

\section{Le cadre épistémique et les schèmes cognitifs liant le spatial au social}

\section{La dimension identitaire de l'espace}

Aujourd'hui, selon notre thèse, l'analyse du fait social et du fonctionnement sociétal la plus répandue dans les courants en vue des sciences sociales procède d'un éloignement de ces sciences vis-à-vis des échelles de lecture du monde social qui étaient hier hégémoniques. Ces lectures d'hier étaient, soit macroscopiques, soit microscopiques. Elles imposaient une appréhension de ce monde en termes d'explications structurelles ou, à l'inverse, atomistiques.

Mais ce qu'elles avaient en commun, c'est qu'elles ne prenaient pas en compte la dimension spatiale du réel : pour le marxisme, en effet, seuls comptent les rapports et modes de production, cependant que pour l'individualisme méthodologique seul l'individu rationnel existe. Or, l'échelle de lecture en vigueur aujourd'hui est de type mésoscopique et cela, tant dans l'ethnométhodologie, la phénoménologie herméneutique, l'interactionnisme symbolique, la psychologie de l'environnement, la sociologie pragmatiste... Dans cette appréhension du réel par interactions et agrégats situés, l'espace représente un facteur constitutif, requérant à ce titre une pleine attention.

Toutefois, il nous semble que cette focalisation sur l'échelle mésoscopique ne constitue pas réellement un ralliement de l'épistémè française à une lecture processuelle et

31. Dépliant «Les projets par quartiers, Vernay-Verchères, La rénovation urbaine du quartier », ANRU, Grand Lyon, Vaulx-en-Velin, fév. 2011. 
pragmatiste (conforme, par exemple, à l'interactionnisme symbolique proposé par E. Goffman, pour lequel l'espace n'est qu'un simple opérateur d'interaction), mais qu'au contraire, et paradoxalement, elle réintroduit à l'échelle locale une pensée hypostasiante et holistique (même si cela procède d'un micro-holisme). Celle-ci attribue à l'espace, entendu comme «lieu ${ }^{32}$ et "territoire ${ }^{33}$, une dimension identitaire, voire une portée ontologique (reprenant en cela une conception heideggérienne). Cette conception phénoménologique de l'espace se traduit par des expressions comme «lien social » ou "vivre ensemble » dans le discours scientifico-politique actuel, ce qui redéfinit par un changement d'échelle ce que nous appelons «cohésion sociale» et "intégration sociale ${ }^{34}$. Il s'agit là de la réaffirmation d'une pensée substantialiste, certes désindexée du paradigme national (un paradigme qui fut longtemps dominant dans les sciences sociales françaises : pensons à la «solidarité organique » et à la «cohésion sociale » comprises dans la sociologie durkheimienne au sens de "conscience morale nationale »). Ainsi, dans cette pensée substantialiste, ce ne sont plus «La Société », "La Nation » qui existeraient comme substance ou essence, mais c'est le milieu (comme agrégation d'un lieu, d'un groupe et des liens internes au groupe et au lieu) qui constitue le substrat du social. C'est donc la figure, du « Nous, ici » qui s'impose cognitivement et qui, de facto, se conçoit réalisée au moyen de sa spatialité et saisissable au travers de cette dernière.

Cette opération de substantification du social par le lieu correspond au fait que l'épistémè nationale régissant les sciences sociales françaises soumet celles-ci à un surmoi ou à un idéal substantialiste. Et si ce dernier perd de sa crédibilité, pour autant ses adeptes ne se résignent pas réellement à la domination d'un paradigme pragmatiste, processuel, utilitariste (qu'ils qualifient en un mot de « libéral »).

\section{Du substantialisme à la substantification}

Ce refus d'une conception du social sans point dur, sans fondement ni englobement des individus dans un Tout; qui plus est dans un Tout dont le Politique peut se dire l'instaurateur et le garant, engendre une ruse ou une confusion: alors que le pragmatisme et l'interactionnisme symbolique récusent l'opposition entre holisme et individualisme et entendent dépasser le clivage entre lecture macroscopique et microscopique, le tournant géographique des sciences sociales françaises se traduit par l'adoption d'une mésoscopie, qui se mue bien vite en mésologie, elle-même nourrie du mythe (voire de la mystique) du «milieu» et du «lieu»: un lieu-milieu considéré comme générateur d'identité et producteur d'un sentiment d'appartenance. En un mot, via le mythe du lieu, facteur d'identification (et non d'individualisation, car ce n'est pas l'ipséité, mais l'identité - le fait d'être identique à d'autres, d'avoir quelque chose en commun - qui est mise en avant), c'est en fait un paradigme "socialiste » (au sens premier du terme, s'opposant à «individualiste», comme au sens second, désignant la valorisation de la socialisation sur la privatisation) qui se réaffirme. Ainsi, c'est une pensée hypostasiante qui se reformule.

Prenons l'exemple de "l'effet de quartier», postulé par les politiques sociales urbaines américaines et françaises. Si, pour les Américains, un effet de quartier négatif existe en certains lieux ghettoïsés, c'est parce que les interactions entre les habitants de ce quartier les enferment dans des comportements déviants, dans une culture de la pauvreté et de la marginalité, alors que, pour les Français, ce sont les espaces en eux-mêmes qui sont " pathogènes et criminogènes » (selon une formulation utilisée par Michel Rocard lors de la création du ministère de la Ville) indépendamment des relations sociales tissées entre les habitants.

32. P. Genestier, 1999.

33. P. Genestier, 2009.

34. Aujourd'hui, il ne s'agit plus de penser l'intégration sociale comme le fait de s'intégrer à un tout préexistant mais aussi de contribuer à un travail d'intégration de ce tout devenu incertain et fragile. De même, la cohésion de la Société ne relève plus d'une conscience morale partagée productrice d'une identité forte et unanime (de type national), mais d'un processus, jamais achevé, de rapprochement, d'intercompréhension et de reconnaissance mutuelle. On assiste alors, avec l'abandon de l'idée de Société, à l'émergence d'une nouvelle représentation collective de la vie sociale, liée au processus d'individuation, dans laquelle la cohésion liée au capital social et à la confiance dans les interactions vient remplacer l'intégration, au sens classique du terme. 
Ainsi, il existerait bel et bien un « effet de quartier » ne découlant pas uniquement de la structure sociale du quartier ${ }^{35}$. Il en découle qu'aux États-Unis et au Royaume-Uni, les politiques people mises en œuvre par l'intervention publique en faveur des quartiers défavorisés passent par du Community Organising, cependant que les politiques place ${ }^{36}$ qui sont appliquées en France entendent « remettre à la norme » des quartiers en organisant la «requalification des lieux », la «mixité sociale » afin de produire une «banalisation urbaine $»^{37}$.

Autre exemple : l'idéal français de Solidarité, tel qu'il est énoncé par la République et la sociologie durkheimienne, conçu comme appartenance à une même et unique Communauté (civique et surtout pas ethnoculturelle), ressurgit aujourd'hui avec l'emploi récurrent dans les discours scientifico-politiques du syntagme " mixité sociale ». Mais cet objectif de mixité sociale n'est rien d'autre que la déclinaison à l'échelle locale du mythe de Communauté politique qui transcende la diversité des identités individuelles et groupales. Ce syntagme témoigne d'un idéal holiste et d'une grammaire substantialiste, et donc anti-processuelle, voire antilibérale, régissant les appréhensions et les raisonnements.

Ainsi, le constat de l'absence de mixité dans les Zones Urbaines Sensibles conduit à la rénovation de ces quartiers, c'est-à-dire à la démolition d'un bon nombre de logements très sociaux. Cependant, si l'on adoptait une lecture processuelle en accordant attention aux trajectoires résidentielles des habitants de ces quartiers, on s'apercevrait que, du fait du renouvellement fréquent des locataires, ces quartiers jouent un rôle non négligeable de refuge et de tremplin, si bien qu'il est très dommageable de les " rénover ", c'est-à-dire de les détruire.

Il apparaît donc que, dans l'épistémologie nationale française (ou dans ce «nationalisme épistémologique et méthodologique », pour reprendre l'expression d'U. Beck, 2006), la conception de la Société (avec un S majuscule, comme l'écrivent F. Dubet et D. Martucelli, 1998) est de nature holistique et substantialiste : il existe un Tout, supérieur à la somme de ses parties, incitant les individus à se penser en termes de "Nous », et qui est doté de suffisamment de consistance interne pour demeurer lui-même sur une longue période tout en déterminant ceux qui lui appartiennent. Bien sûr, ce modèle épistémologique n'est pas isolé et autonome. Il participe de ce que P. Rosanvallon (2004) appelle «le modèle politique français ", c'est-à-dire ce mélange - pour le dire en termes anthropologiques - de prémisses ontologiques, d'options axiologiques, de schèmes étiologiques et de visions sotériologiques qui irriguent l'idéologie propre au monde institutionnel, indépendamment des adhésions partisanes de ses membres, et qui guident et motivent l'imaginaire de l'action publique.

\section{De la Société-République à la cohésion-démocratie?}

Or, ce modèle politique est pourtant aujourd'hui remis en question, notamment dans sa dimension épistémologique :

«Longtemps, j'ai cru vivre en société. J'ai même pensé que la sociologie consistait à étudier cette société, à en étudier la nature, les mécanismes, les ressorts, et pourquoi pas, les «lois ». » ${ }^{38}$

Selon cet auteur, nous sommes à l'heure de la psychologisation et de la désinstitutionalisation des rapports sociaux, ce qui révoque de facto le paradigme de La Société. De même, U. Beck ${ }^{39}$ affirme que la nation n'est qu'une des dimensions de l'identité collective, pas plus pertinente que d'autres pour aborder la question des différences et des groupes sociaux au sein d'un monde globalisé. Pour sa part, J. Urry a

35. Voir, par exemple, J.-Y. Authier, 2007.

36. La distinction entre une politique de la ville «people» et une politique «place » a été développée par J. Donzelot, 2003.

37. R. Epstein, 2013.

38. F. Dubet, 2009, p. 7.

39. U. Beck, 2006. 
consacré son livre Sociology beyond Societies (2001) à la formulation d'un paradigme alternatif à celui fondant la conceptualisation par la sociologie du fait sociétal à partir de la nation. Ce qui le conduit à proposer que la sociologie du contemporain repose sur des concepts liés aux phénomènes de déplacement et de communication, de réseaux et de flux puisque les facteurs de mobilité constitueraient la matrice du lien social dans le monde transnationalisé.

Si une part de la sociologie se réfère à l'échelle de la mondialisation (et de ce fait consent, voire acquiesce à une perte d'influence de l'instance politique indexée à la souveraineté des États nations) pour se confronter explicitement à la crise affectant l'ancienne conception nationale-holistique du fait sociétal, une autre part réagit en fonction de ce qu'elle voudrait que l'action publique puisse continuer à être et à faire. Cette autre part tente de résister à cet âge de «l'écume» qui se substitue à celui «des sphères » englobantes qui assuraient les appartenances, comme l'analyse P. Sloterdijk (2012), en vue de contrecarrer cette société « liquide», telle que la qualifie Z. Bauman, et qui lui apparaît surtout comme la liquidation par le néolibéralisme de la capacité des institutions publiques à agir sur les structures sociales. Les sociologues français, n'entendant pas découpler leur lecture des phénomènes sociaux et leur philosophie du social, qui est aussi une philosophie du souverainisme politique, s'inspirent alors discursivement de la sociologie interactionniste et pragmatiste anglo-saxonne, mais pour tout de suite s'en écarter en réduisant la part de liberté individuelle que cette dernière postule. Là s'exprime une volonté de souveraineté, un attachement à une conception instaurationniste ou constructiviste du politique $\mathrm{e}^{40}$.

Cette campagne de sauvegarde d'une représentation substantielle et consistante du social, prémisse et réquisit à une conception activiste de $l^{\prime}$ agir politique ${ }^{41}$, passe par une reformulation, par simple substitution, des catégories d'objets sur lesquels et au travers desquels va s'exercer l'action publique. Dans le paradigme français, à La Société (conçue comme Communauté nationale productrice d'identité, de valeurs et de règles) se substituent des conditionnements, des prescriptions, qui n'opèrent plus par le poids d'une conscience morale intériorisée mais, par des dispositifs symboliques, des mécanismes techno-économiques d'échanges et par des agencements socio-spatiaux aux effets éthologiques. Ce qu'il y a de commun à ces deux lectures, c'est que, in fine, le sujet reste identifié, attaché, déterminé, si bien que sa part de singularité et d'autonomie s'en trouve réduite et que ce sujet reste tributaire d'un destin commun élaboré en dehors de lui, par une "volonté générale », ou par une "cohésion » qui lui sont supérieures. Et il semble que, dans l'imaginaire institutionnel français, ces instances d'englobement et d'appartenance, qu'elles soient macroscopiques ou mésoscopiques, disposent d'une légitimité déniée à l'individu, toujours suspecté d'être anomique car trop individué et alors borné par et dans ses intérêts propres.

Ainsi, le mouvement récent de la pensée française a consisté à passer de l'intégrationappartenance abstraite à l'inclusion-insertion concrète, et de l'imprégnation par la collectivité à un conditionnement par le lieu-milieu. Il y a eu un glissement du paradigme de l'identification à et par la Nation, assurée par l'assignation à un rôle et à un statut ainsi que par l'intériorisation d'un ordre moral moniste-universaliste jusqu'au paradigme $\mathrm{d}$ 'une acculturation réciproque par côtoiement. Et dans ce déplacement, on observe à la fois une focalisation sur l'échelle du lieu et une substantification des idéaux (les « aménités urbaines » et $\mathrm{l}^{\prime}$ « urbanité » apparaissent comme des substituts à la « concorde sociale » qui avait été posée en tant qu'idéal régulateur au sein de la vie en Société, telle que l'avait définie E. Durkheim).

40. Au sens où Karl Popper (1971) parle du « constructivisme rationaliste français », expression d'un imaginaire social-historique faisant de la société un artefact façonné par une volonté extérieure et supérieure aux hommes et aux groupes qui la constituent.

41. Il semble que la vision instaurationniste du politique reste tributaire du schème théologico-politique porté par la mythologie grecque comme par les trois monothéismes : que ce soient les Titans Prométhée et Epiméthée ou que ce soit Yahvé, c'est en modelant la glaise du limon que La Puissance donne naissance à l'Humanité. Il faut donc concevoir le social comme une pâte lisse et meuble à disposition d'une force faconnante. Si, à l'inverse, la glaise contient trop de cailloux, de scories, ou si elle se transforme en sable sec et pulvérulent, alors le modelage est impossible. 
Toutefois, dans cette double translation, à la fois référentielle et scalaire, est maintenue l'idée d'une possible cohésion du collectif et d'une action publique opérante en faveur de celle-ci. Il en découle que l'action publique peut prétendre sauvegarder sa nature substantielle, et cela selon trois sens de ce terme: elle a besoin de réalités quasi substantielles pour y exercer une force (selon un paradigme physicaliste) de transformation, elle peut revendiquer d'impacter la substance même des choses et elle peut se référer à une conception substantielle du Bien, par opposition à sa conception procédurale (anglo-saxonne), et donc relativiste. Le modèle politique français peut ainsi penser persister dans son être.

\begin{abstract}
Résumé
Comment, au cours des dix dernières années, en est-on venu à appliquer une politique de « rénovation urbaine » consistant à démolir massivement des immeubles HLM, alors que dans le même temps la pénurie de logements pour les populations modestes ne cessait de s'accroître? Les réalités de terrain (dégradation du bâti, obsolescence urbanistique, marginalité des quartiers) n'expliquant pas réellement les choix qui ont été faits par les pouvoirs publics en la matière, ce texte s' efforce de montrer que la réponse à cette énigme se trouve dans l'examen des cadres cognitifs et des instruments discursifs qui ont été engagés pour appréhender ces quartiers. Ainsi, ce sont les catégories de désignation, les schèmes de raisonnement et l'appareillage conceptuel des sciences sociales contemporaines qui, en amalgamant les réalités sociales et spatiales, ont prédéfini et formaté le type d'actions ressenties comme nécessaires et efficaces par les acteurs publics.
\end{abstract}

\title{
Bibliographie
}

Authier Jean-Yves, BACQuÉ Marie-Hélène, GuÉRIN-PACE France, Le quartier. Enjeux scientifiques, actions politiques et pratiques sociales, Paris, La Découverte, 2007.

BACOT Paul, RÉMI-GIRAUD Sylvie, Mots de l'espace et conflictualité sociale, Paris, L'Harmattan, 2007.

BAUMAN Zygmunt, La société assiégée, Éditions du Rouergue, 2005.

BECK Ulrich, Qu'est-ce que le cosmopolitisme? Paris, Aubier, 2006.

Chalas Yves, «L'imaginaire aménageur ou le complexe de Noé », Les annales de la Recherche Urbaine, $\mathrm{n}^{\circ}$ 42, 1989, pp. 66-73.

Donzelot Jacques, Mével Catherine, WyveKens Anne, Faire société, Paris, Seuil, 2003.

Dubet François, MARTUCELli Danilo, Dans quelle société vivons-nous ?, Paris, Seuil, 1998.

DUBET François, Le travail des sociétés, Paris, Seuil, 2009.

DufOuR Michel, Argumenter. Cours de logique informelle, Paris, A. Colin, 2008.

EPSTEIN Renaud, La rénovation urbaine. Démolition-reconstruction de l'Etat, Paris, Presses de Sciences Po, 2013.

GENESTIER Philippe, «Le sortilège du quartier. Quand le lieu est censé faire le lien. Cadre cognitif et catégorie d'action publique ", Les Annales de la Recherche Urbaine, $\mathrm{n}^{\circ}$ 82, mars 1999, pp. 142-153. 
GENESTIER Philippe, OuARDI Samira et RENNES Juliette, «Le paradigme localiste au secours de l'action publique démocratique ", Mots. Les langages du politique [En ligne], 83 | 2007, mis en ligne le $1^{\text {er }}$ mars 2009, consulté le 6 novembre 2017. URL: http:// mots.revues.org/907

GENESTIER Philippe, «Espace et identité narrative: les fonctions identificatoire et instituante de l'espace en question », dans BAUDIN G. et BONNIN P. (dir.), Faire Territoire, Paris, Éditions Recherches, 2009.

GENESTIER Philippe, "L'urbanophilie actuelle, ou comment le constructionnisme politique se réaffirme en s'indexant à la ville », dans SALOMON CAVIN J. et MARCHAND B., Antiurbain. Origines et conséquences de l'urbaphobie, Lausanne, Presses polytechniques et universitaires romandes, 2010.

GENESTIER Philippe, «Percevoir, dénommer et évaluer la ville pour agir sur elle: les catégories de pensée et le raisonnement de la politique de la ville à l'œuvre", dans VICTOR A., Penser et agir Contextes psychosociologique, psychologique, spatial et écologique Tome 2, Paris, Le Manuscrit, 2011.

LAKOFF George et JOHNSON Mark, Les métaphores dans la vie quotidienne, Paris, Les Éditions de Minuit, 1985.

POPPER Karl, La société ouverte et ses ennemis, Paris, Seuil, 1971.

RosanVALlon Pierre, Le modèle politique français, Paris, Seuil, 2004.

SLOTERDIJK Peter, Écumes Sphères III, Paris, Pluriel, 2012.

TOULmin Stephen E., [The Uses of Argument. Cambridge, Cambridge University Press, 1958], Les usages de l'argumentation, Paris, PUF, 1993.

URRY John, Sociology beyond Societies, New York, Routledge, 2001. 


\title{
Les métaphores favorisent-elles la compréhension d'une théorie scientifique? Le cas des images darwiniennes de "lutte pour la vie" et de "sélection naturelle»
}

\author{
Antonello LA VERGATA \\ Professeur d'Histoire de la philosophie, \\ Département d'Études linguistique et culturelle, \\ Université de Modène et de Reggio d'Émilie \\ Extrait de : Xavier LAFON, Les langues savantes, Paris, \\ Édition électronique du CTHS (Actes des congrès des sociétés historiques et scientifiques), 2017. \\ Cet article a été validé par le comité de lecture des Éditions du CTHS dans le cadre de la publication \\ des actes du $139^{\mathrm{e}}$ Congrès national des sociétés historiques et scientifiques tenu à Nîmes en 2014.
}

\section{Métaphores et méprises}

Dans la communication scientifique, on pense généralement que les métaphores jouent un rôle essentiellement didactique. Leur domaine, croit-on, est celui de la vulgarisation. En effet, nous gardons tous le souvenir des jours à l'école où l'on nous apprenait les lois de la physique par l'intermédiaire de boules qui roulaient et s'entrechoquaient sur la surface d'un billard. Le recours à des images tirées de l'expérience quotidienne devient d'autant plus nécessaire que la science se spécialise, se mathématise et s'éloigne du plan intuitif du sens commun. Les scientifiques eux-mêmes utilisent des métaphores, des analogies, des expériences mentales pour mieux expliquer leurs idées les plus nouvelles ou difficiles à comprendre, ou pour mieux les fixer dans l'esprit des allocutaires : il suffit de rappeler le «petit diable de Maxwell » ou le "chat de Schrödinger», ou la célèbre phrase d'Einstein « Dieu ne joue pas aux dés avec l'univers ».

Dans des cas comme ceux que nous venons de mentionner, on dirait normalement qu'il s'agit de communication scientifique, et non de vulgarisation proprement dite. Mais la différence entre les deux est-elle toujours aussi nette qu'on le croit? Après tout, il n'y a pas seulement une vulgarisation "verticale », du spécialiste au non-initié, il y en a aussi une «horizontale», du spécialiste d'un domaine particulier au spécialiste d'un autre domaine : un astrophysicien peut bien être aussi profane en biologie moléculaire qu'un avocat ou un ingénieur. Pendant au moins un siècle les morphologues, les paléontologues et les naturalistes « en plein air » qui étudiaient les populations animales et végétales dans leurs habitats ont entendu trois choses différentes par le simple mot « espèce ». Même entre des spécialistes de la même matière il peut y avoir des problèmes de communication, voire de compréhension, très importants : est-on sûr qu'un physicien théorique et un physicien pratique parlent toujours le même langage ? Pour ne rien dire de deux philosophes qui habitent deux bureaux contigus de la même faculté... Ce n'est qu'une illusion de profane que de croire que les «spécialistes de l'autre côté » doivent s'entendre nécessairement entre eux.

Mais les métaphores ne servent pas que pour parler, elles servent aussi pour penser, et cela est vrai aussi bien pour les scientifiques que pour le reste du monde. Parfois on les construit, comme on fait pour les modèles, aujourd'hui mathématiques, qui simulent la réalité pour mieux la manipuler. Mais pour la plupart elles sont tirées, presque 
inconsciemment, du contexte historique, culturel et linguistique dont l'usager fait partie. Cependant, elles s'avèrent souvent une source de celles qu'on pourrait définir, d'une façon un peu expéditive, des «méprises", car elles multiplient l'ambiguïté des mots et décèlent l'ambiguïé qui se cache dans l'expérience quotidienne, dans le sens commun et dans les images les plus familières.

L'histoire des sciences est pleine de ces «méprises ». En rechercher les causes est d'une importance vitale pour l'historien des idées, du moins pour celui qui s'est débarrassé de l'image positiviste d'une histoire des sciences réduite au simple enregistrement des découvertes faites par «ceux qui avaient raison ». Dans l'exposé qui suit, j'ai essayé de répondre à des questions à première vue circonscrites, mais qui se sont avérées tentaculaires: comment a-t-on reçu les métaphores darwiniennes de la "lutte pour l'existence » et de la "sélection naturelle» ? Ont-elles aidé la compréhension des concepts-clé de la théorie de Darwin ? Les méprises n'ont-elles produit que le rejet de la théorie?

\section{Les métaphores dans l'œuvre de Darwin}

Il y a beaucoup de métaphores dans les écrits de Darwin. Parmi elles, on trouve l' «arbre de la vie »; une documentation fossile comparée à un texte dont ne sont conservées que quelques pages, et de ces pages seulement quelques lignes fragmentaires; la «division du travail » au sens physiologique de Milne Edwards, mais surtout au sens écologique, et tout à fait nouveau, lié au " principe de divergence des caractères »; la puissance de la pression démographique comparée à la "force de dix mille coins » qui appuient pour s'enfoncer dans $l^{\prime}$ «économie de la nature», en sapant d'autres ${ }^{1}$. Le mot même $\mathrm{d}^{\prime}$ «évolution » est une métaphore. C'est une métaphore dangereuse : elle fait penser au déroulement de quelque chose qui était enroulé sur lui-même, à l'explication de ce qui était implicite, au déploiement d'une potentialité, au développement de quelque chose d'enveloppé, au déroulement d'un parchemin déjà écrit... Ce n'est pas pour rien qu'en biologie "évolution » a eu longtemps un sens embryologique, dans le cadre de la théorie de la «préformation des germes". Ce n'est pas à Darwin qu'on doit l'usage $\mathrm{d}^{\prime}$ « évolution » dans le sens aujourd'hui courant (dans l'Origin il utilise "descendance avec modifications » et emploie seulement une fois, et dans la conclusion, le verbe to evolve), mais à T. H. Huxley et surtout à Spencer. Pour celui-ci, le terme avait un sens dangereusement large, au point de pouvoir embrasser le développement embryonnaire comme la formation du système solaire, l'histoire du langage autant que celle des sociétés. Il utilisait dans ce sens large aussi le terme de "progrès » (progress), pas très loin étymologiquement d'evolution. Et l'on connaît les conséquences, pas uniquement en biologie, de la superposition - une véritable identification souvent - des idées d'évolution et de progrès.

Mais bien sûr les deux métaphores darwiniennes les plus célèbres sont le struggle for existence (ou struggle for life ${ }^{2}$ ) et la « sélection naturelle». En elles est concentré le noyau des innovations scientifiques et conceptuelles irréversibles opérées par Darwin.

\footnotetext{
1. Darwin Charles, Notebook D, p. 106. L'image revient dans la première édition de l'Origin, mais disparaît dans les suivantes, peut-être - a-t-on pensé - du fait de sa violence, incompatible avec le cadre généralement déiste dans lequel Darwin présentait sa théorie au public.

2. Darwin les utilise indifféremment. Comme on le sait, survival of the fittest est en revanche une invention de Spencer.
} 


\title{
Il y a lutte et lutte
}

\author{
Darwin précisait :
}

« Je ferai observer au préalable que j'utilise ce terme [lutte pour l'existence] en un sens large et métaphorique comprenant la dépendance d'un individu à l'égard d'un autre, et comprenant (ce qui est plus important) non seulement la vie de l'individu, mais le succès avec lequel il laisse une progéniture. $»^{3}$

Des exemples suivaient, qui étaient très clairs. Peut-être aurait-il dû dire " "aussi" ou "surtout" en un sens large et métaphorique", vu qu'il n'excluait pas complètement l'acception littérale. Lui-même, en effet, se servait quelquefois d'expressions plus «militaires » : par exemple, pour donner une idée de l'énorme complexité des relations écologiques, il dit que «des batailles au sein d'autres batailles doivent se produire continûment avec un succès variable $»^{4}$.

Les matériaux rassemblés par Darwin dans la rubrique «lutte pour l'existence » de ses archives personnelles en vue de ses publications comprennent des choses très différentes : exemples d'obstacles à l'augmentation des populations végétales et animales, acclimatation des espèces immigrées, distribution géographique, extinctions causées par les envahisseurs, chaînes alimentaires, cas d'abondance ou de rareté d'espèces du même genre dans une aire déterminée, jusqu'aux exemples de ce que d'autres définiraient comme «coopération » ou «secours mutuel » et opposeraient à la lutte pour l'existence dans l'acception littérale!

«Ce sont ces idées différentes, qui d'ailleurs sont liées, que je réunis pour plus de commodité sous l'expression générale de lutte pour l'existence. $»^{5}$

Ce concept consistait donc en un entrelacement d'images, il avait un caractère non seulement métaphorique, mais «plurimétaphorique » et polysémique, et c'est justement grâce à cela qu'il a servi à Darwin pour penser la complexité des interactions écologiques et des mécanismes de l'évolution. Ses métaphores, dont celle-ci, n'étaient pas seulement des instruments rhétorico-linguistiques, mais aussi des images productrices de pensée : en plus d'une fonction didactique et descriptive, elles revêtaient aussi une fonction autodidactique, pour ainsi dire ${ }^{6}$.

La nature métaphorique de la lutte dont parlait Darwin aurait pu être claire à quiconque avait lu le livre avec attention. Pourtant, les méprises furent nombreuses, et pas seulement de la part des moins avertis. La raison principale en fut la nouveauté de l'image darwinienne de la nature et la tentative de la ramener aux schémas de pensée précédents. Cette nouveauté ressort, si l'on fait une comparaison avec l'usage qui a été fait, avant comme après Darwin, de l'image, antique en soi, de la « guerre » entre les êtres vivants. Linné et son école, par exemple, en arrivaient à décrire la nature comme une atrox laniena ou un bellum omnium contra omnes, aussi pour démontrer, par un habile retournement rhétorique, comment de tout cela jaillit, par un dessein divin admirable, un «équilibre de la nature» providentiel fondé sur une sage "économie» (que des métaphores, comme on voit). Mais, comme tout le monde jusqu'à Darwin, ils entendaient la lutte interspécifique, autrement dit entre espèces différentes. C'est seulement avec Darwin que la lutte intraspécifique devient le centre de l'attention et le cœur de l'évolution. Le mot « darwinisme » signifia des choses différentes pour des personnes différentes.

3. C. Darwin, L'Origine des espèces, p. 343.

4. Ibid., L'Origine des espèces, p. 353-354.

5. Ibid., p. 344.

6. L'étude la plus ample et détaillée des métaphores darwiniennes se trouve dans E. Manier, The Young Darwin and His Cultural Circle. 
Il est en outre connu désormais qu'il ne fut pas refusé seulement par les ignorants ou par des personnes aveuglées par l'odium theologicum: les savants les plus éminents, comme Louis Agassiz, Karl Ernst von Baer, Claude Bernard, Rudolph Virchow, Louis Pasteur, eurent à son égard des réactions qui allaient de la polémique ouverte à la méfiance, du silence indifférent au sarcasme. Le langage même de L'Origine des espèces fit l'objet de critiques, parmi lesquelles il faut signaler les invectives de Pierre Flourens, secrétaire perpétuel de l'Académie des Sciences, membre de l'Académie française, professeur au Muséum d'histoire naturelle et au Collège de France :

«Quel langage prétentieux et vide! Quelles personnifications puériles et surannées!

O lucidité ! O solidité de l'esprit français, que devenez-vous ? »

Comme on devait s'y attendre, un nombre énorme de lecteurs entendit la lutte dans l'acception la plus littérale, celle qu'Huxley définit comme un « combat de gladiateurs ${ }^{8}$.

«Les Darwinistes actuels - écrivait le biologiste français Yves Delage - se représentent les individus dans la nature comme des duellistes de force un peu inégale et croient que les plus avantagés résisteront seuls, de même que les duellistes qui ont, si peu que ce soit, plus de sang-froid, de méthode, de force dans le poignet, de vivacité dans les mouvements, l'emporteront en moyenne sur leurs adversaires. C'est là une idée très fausse. »"

La littérature est pleine $\mathrm{d}^{\prime}$ " espèces vaincues" et $\mathrm{d}^{\prime}$ " espèces victorieuses », $\mathrm{d}^{\prime}$ " exterminations », de "luttes entre les forts et les faibles », "élus » et " délaissés », «domination brutale et féroce de la force », "destruction des inoffensifs par les féroces », «variétés abattues ou fauchées dans la mêlée », «combats et souffrances ». Beaucoup se firent forts de cette méprise pour refuser la théorie darwinienne. Certains crurent la discréditer en demandant comment il pouvait être possible, après de tels massacres, que la vie ne soit pas réduite «à une seule unité » ou, tout au plus, «à quelques espèces victorieuses $\gg{ }^{10}$. Certains se demandèrent comment on pouvait parler de lutte entre les plantes, puisqu' « il $n^{\prime} y$ a pas de lutte là où il $n^{\prime} y$ a pas de volonté ${ }^{11}$. Comment pouvaiton, dirent d'autres, voir dans la lutte un facteur d'harmonie et d'équilibre ? Comment croire que « l'ordre soit sorti du désordre »?

«On a dit que les êtres des divers âges géologiques ont eu, les uns avec les autres, des luttes où les plus forts ont vaincu les plus faibles, de sorte que le champ de bataille est resté aux mieux doués ; ainsi le progrès serait la résultante des combats et des souffrances du temps passé. »

Mais la paléontologie démontre que : «Le monde géologique n’a pas été un théâtre de carnages, mais un théâtre majestueux et
tranquille. »12

Darwin, dirent d'autres, avait une «conception militaire et utilitaire de la vie universelle », expliquée par le heurt des égoïsmes adverses, dans laquelle la théorie entière de la sélection naturelle était «digne de Torquemada » ${ }^{13}$. Tel le zoologue Armand Sabatier :

«Il est clair en effet que le darwinisme donne du gouvernement de ce monde une idée peu flatteuse au point de vue moral, qui est, après tout et sans aucun doute, celui qui importe le plus. Cette domination brutale et féroce de la force, cet anéantissement fatal du faible, cette

7. P. Flourens, Examen du livre de M. Darwin sur l'origine des espèces, Paris, 1864, p. 65.

8. T.-H. Huxley, «The Struggle for Existence in Human Society», p. 200.

9. Y. Delage, L'hérédité et les grands problèmes de la biologie, p. 844.

10. A. La Vergata, L'equilibrio e la guerra della natura, p. 431, 433, 434

11. J.-L. Gray (dir.), Letters of Asa Gray, vol. II, p. 626-627.

12. A. Gaudry, Essai de paléontologie philosophique, p. 30.

13. Ainsi s'exprimèrent le sociologue français Tarde et l'écrivain populiste russe Černyševskij (cités dans Antonello La Vergata, L'equilibrio e la guerra della natura, p. 445, 453. 
négation des droits antérieurs et de la justice, cet appel à la violence, justifiés par la nécessité du progrès, cette idée enfin qu'il n'y a dans la nature que lutte acharnée, que combats impitoyables, que meurtre et spoliation, que la vie et le progrès ne sont possibles qu'à ces conditions, toutes ces considérations en un mot qui représenteraient la direction de ce monde comme appartenant à la force et non à la justice et à l'amour, ne sont que très difficilement compatibles avec l'idée d'un Créateur et Législateur, sage, juste et bon. Était-il possible que les idées de progrès, de perfectionnement qui ont en réalité un caractère de moralité, ne pussent trouver leur réalisation que dans des conditions de la plus évidente immoralité ? » 14

En 1918 encore, Jean Piaget écrivait que «le darwinisme envisagé comme seule explication de l'évolution justifie la guerre», car il voit dans la concurrence entre individus et entre espèces tout le mécanisme de l'évolution, et «la concurrence, c'est la lutte, il n'y a pas de discussion possible ${ }^{15}$.

En revanche, l'image de la lutte eut un large succès en vertu justement de sa supposée truculence. On reconnaissait finalement que dans la nature c'est « le droit du plus fort » qui prévaut, la "guerre d'extermination », s'écriait content le biologiste Oscar Schmidt, qui n'était certes pas un socialiste. Mais le socialiste Edward Aveling, gendre de Marx, écrivait lui aussi: "Le monde est un grand champ de bataille [...] Malheur aux vaincus! $»^{16}$. Le botaniste Asa Gray, que Darwin estimait au point de le mettre au courant de ses théories avant même qu'elles soient rendues publiques, affirmait avec désinvolture que la théorie de Darwin était non seulement fondée sur celle de Malthus, mais aussi sur celle de Hobbes. La sélection naturelle, selon Gray, était "comme la doctrine napoléonienne selon laquelle la Providence favorise les bataillons les plus forts » :

«Le dicton selon lequel " une maison lacérée par les divisions ne peut rester debout" trouve dans la nature son plus flagrant démenti. La guerre civile est ici l'instrument même du progrès. » 17

Le règne organique, écrivait le philosophe américain John Fiske, est « un carnage extraordinaire $»$ :

«Des batailles bien plus sanglantes que Gettysburg et Gravelotte ont été menées sans cesse sur chaque mille carré de la surface habitable de la terre depuis les débuts de la vie ${ }^{18}$.

Le biologiste français Félix Le Dantec publia en 1906 un livre intitulé La lutte universelle (dont le sous-titre était être, c'est lutter, vivre, c'est vaincre) et en 1911 il écrivit:

« La biologie [...] ne nous enseigne que la lutte et la sélection résultant de la lutte [...] L'idée de lutte est inséparable de l'idée de vie [...] La vie est un acte absolument égoïste, et l'être vivant est en lutte contre l'Univers entier, dans lequel il ne conserve sa place qu'au prix de triomphes incessants [...] L'être vivant est seul contre tous, [...] il est l'ennemi de tout ce qui n'est pas lui. $\gg^{19}$

Et ainsi durant des pages et des pages, en voyant une lutte dans les réactions aux variations de température, dans la digestion, dans la défense contre les bactéries, dans tous les aspects de la vie sociale. Et dans la guerre évidemment, qu'il définit en 1917 «la fonction la plus naturelle de l'être vivant » et de l'homme, bienfaisante en ce qu'elle démasque toute hypocrisie morale et religieuse :

«En temps de guerre [...] on dit la vérité, c'est-à-dire qu'on proclame, par nécessité, le droit du plus fort $»^{20}$.

14. A. Sabatier, Philosophie de l'effort, p. 111-112.

15. J. Piaget, «La biologie et la guerre», p. 40.

16. Cités par Antonello La Vergata, L'equilibrio e la guerra della natura, p. 444.

17. A. Gray, Darwiniana, p. 30, 74, 313)

18. J. Fiske, Outlines of Cosmic Philosophy, p. 11, 12

19. F. Le Dantec, L'égoïsme seule base de toute société, p. 2, 61, 63.

20. F. Le Dantec, Savoir!, p. 58. 
Bien évidemment, devant ces fleurs rhétoriques (seulement rhétoriques ?) il n'était que trop facile de s'indigner au nom de la coopération et de l'altruisme en tant que faits naturels, autant sinon plus que la lutte. Kropotkine tonna contre les déformations des darwiniens «bourgeois » aveuglés par l'individualisme et par l'avidité. "Ce qu'ils ont fait de Darwin - écrivit-il - est abominable » :

«Les innombrables continuateurs de Darwin [réduisirent] la notion de lutte pour l'existence à son sens le plus étroit. Ils en vinrent à concevoir le monde animal comme un monde de lutte éternelle entre individus affamés, assoiffés de sang. Et ils firent résonner la littérature moderne du cri de guerre Gare aux vaincus, comme si c'était le dernier mot de la biologie moderne. Et, par intérêts personnels, ils élevèrent la «lutte sans pitié » à la hauteur d'un principe biologique, auquel l'homme doit se soumettre sous peine de succomber dans un monde fondé sur l'extermination réciproque. $»^{21}$

Pendant la Grande Guerre, les biologistes français, qui étaient presque tous lamarckiens, eurent beau jeu de soutenir que l'interprétation littérale et militaire de la lutte pour l'existence était un des éléments constitutifs du germanisme agressif qui avait déchaîné la catastrophe. En insinuant que cette interprétation était plus ou moins inévitable, ils réglaient aussi leur compte avec le darwinisme lui-même, tout en évitant de critiquer directement l'illustre représentant d'un pays alliée 22 .

\section{Effort et épreuve}

Il se trouva beaucoup de darwiniens qui interprétèrent la lutte pour la vie comme un synonyme d' « effort » ou d' « épreuve ». Cette interprétation rencontra la faveur de ceux qui étaient déjà prédisposés, pour des raisons diverses, à voir dans le monde naturel (et humain) un théâtre, non d'un combat de gladiateurs au sens littéral, mais d'une tension biologico-morale vers le progrès. On se trouve donc ici en présence de quelque chose d'apparemment paradoxale : les «méprises » n'eurent pas toujours pour conséquence le refus du darwinisme. Dans de nombreux cas, au contraire, elles en favorisèrent l'acceptation, ou du moins la pénétration dans des milieux où l'évolutionnisme rencontrerait des résistances très fortes, par exemple dans les courants d'origine calviniste, et parmi eux ceux qui étaient particulièrement sensibles au message moral du self help et du no pain no gain.

Le drame entier de l'évolution pouvait être vu dans un cadre moral qui lui conférait le sens spirituel d'une ascension continuelle et progressive, même à travers la lutte, l'effort, la douleur et la destruction, au couronnement dans l'esprit humain. Il y avait continuité entre le monde biologique et le monde spirituel. Le processus cosmique avait un sens, tout comme les souffrances des êtres vivants ${ }^{23}$.

Henry Drummond, théologien et professeur de sciences naturelles à Glasgow, au Theological College de la Free Church d'Écosse, dans Natural Law in the Spiritual World (1883) interpréta la lutte pour l'existence comme une tension universelle, un effort du monde vivant pour accéder à des formes toujours supérieures, à travers l'épreuve avec les adversités. Dans le monde humain l'évolution prend la forme du progrès spirituel à travers l'exertion et le travail. Dans la nature comme dans la société, quand les stimuli et la tension sont absents, se produit une dégénérescence. Drummond citait l'exemple de la Sacculine, un organisme qui jeune ressemble à une écrevisse, avant de se changer en parasite du bernard-l'ermite, se réduisant à un simple sachet rempli d'œufs, qui absorbe

21. P. Kropotkine, L'entr'aide, p. 4.

22. A. La Vergata, «Lamarckisme et solidarité»; J.-M. Bernardini , Le darwinisme social en France (1859-1918), p. 367-388 ; A. La Vergata, «Evoluzionismo alla francese contro darwinismo alla tedesca».

23. Le même Sabatier que nous avons vu réfuter la conception brutale de la lutte percevait dans l'évolution, correctement entendue, une sorte $d^{\prime}$ «inquiétude biologique», comme «le pressentiment d'une fin supérieure» qui rachetait l'apparente matérialité du processus entier. 
la nourriture de son hôte à travers des sortes de racines. Sa dégénérescence, poursuivaitil, est une punition pour deux fautes :

«Avant tout, il a ignoré l'évolution ; en second lieu, ce qui est pratiquement la même chose, il a enfreint la loi du travail. Donc la vengeance de la Nature était nécessaire. »

Pareillement, l'extraordinaire fécondité de la nature tropicale dispense les natifs chanceux seulement en apparence - de la nécessité de faire des efforts : voilà pourquoi $l^{\prime}$ Afrique est une terre d'oisifs et de fainéants ${ }^{24}$.

De semblables réinterprétations n'allaient pas du tout dans la direction d'une désanthropomorphisation de la métaphore de la lutte. Bien au contraire :

" "Lutte pour l'existence" - lisons-nous chez un autre darwinien - est une formule qui comprend toutes les réactions et les efforts (endeavours) des créatures vivantes face aux difficultés et aux limitations. ${ }^{25}$

En effet, la caractéristique fondamentale des organismes est l'«effort de bien-être (endeavour after well-being)». "L'être vivant a sa propre volonté, une volonté de vie ». L'organisme vigoureux est caractérisé par la self-assertiveness :

«Un organisme vigoureux a tendance à être agressif. Il joue des coudes dans la foule, en bousculant ses voisins. La plante elle aussi fait pression et obstacle, piège et étrangle, pique et tue $[\ldots]$ Il y a lutte pour l'existence partout où l'être vivant fait pression contre les conditions qui le limitent, partout où des êtres vivants, attaquant et esquivant, changeant et étant changés, disent de quelque manière : Nous vivrons! L'essence de l'être vivant est de s'imposer. S'il ne peut faire autrement, il se multiplie. La vie est effort, elle se développe, s'entremêle, proteste contre ses limites. Un être vivant menace les autres, rivalise avec les autres, mange les autres. Pour indiquer tous ces heurts et ces évitements entre les êtres vivants et leurs limites nous utilisons la formule "lutte pour l'existence" [...] Si la nature a quelque chose à dire à l'homme, ce quelque chose est le mot "effort" (endeavour) [...] La nature est tout entière pour l'efficacité et contre la paresse. ${ }^{26}$

Lutte contre la proie et contre le prédateur, avec des individus de la même espèce, avec le milieu physique, pour l'accouplement, pour défendre sa progéniture: lequel de ces efforts est une « lutte pour l'existence »?

«Chacun d'eux. En effet le vrai sens du terme se trouve non dans la représentation de tel ou tel type de lutte ou d'effort, mais plutôt dans l'idée générale que les organismes vivants s'affirment contre les restrictions et les difficultés, certainement dues aussi aux contrecourants directs du même groupe, voire de la même famille, mais pas seulement à cela. Le progrès dépend de bien plus que d'une querelle pour son bout de pain. La lutte pour l'existence est beaucoup plus qu'une lutte à mort aux marges de la subsistance; elle comprend tous les multiples efforts pour soi ou pour les autres entre les extrêmes de l'amour et de la faim ; elle embrasse tous les efforts du compagnon pour sa compagne, du père pour l'enfant, du parent pour le parent et toutes les circonstances particulières de l'affirmation de soi. Pour de nombreux animaux l'existence signifie le bien-être d'un autre organisme lié aux liens sociaux ou de parentèle dans un contexte social ; l'égoïsme n'est pas satisfait tant qu'il ne devient pas altruiste. ${ }^{27}$

Même la solidarité, la coopération, l'altruisme sont une lutte pour l'existence. En effet, tant la «lutte pour soi » que la «lutte pour les autres» sont des réactions de l'organisme aux limitations :

24. H. Drummond, Tropical Africa, p. 56 ; Natural Law in the Spiritual World, p. 344, 350. L'exemple du Sacculina était tiré du travail avec lequel Edwin Ray Lankester en 1880 avait porté l'attention du monde scientifique sur le concept de "dégénérescence », dont il faisait une loi valide même dans l'histoire humaine (cf. A. La Vergata, La sacculina e il « Regno dell'uomo »).

25. J.-A. Thomson, Darwinism and Human Life, p. 13

26. J.-A. Thomson, Darwinism and Human Life, p. 83, 87-88, 92.

27. Ibid., p. 92. 
«Le monde est, oui, la demeure du fort, mais c'est aussi la demeure de nombreux très faibles qui se rattrapent par l'amour de la force qui leur manque. »

La coopération n'est pas le contraire, mais :

«L'autre face de la lutte pour l'existence [...] où le vaste concept de "lutte" - ou de "réaction aux manques" - inclut celui de secours mutuel. $»^{28}$

Plus ou moins sur cette ligne on trouve un biologiste non darwinien, mais lamarckien, le français Edmond Perrier, défenseur de la «solidarité » comme loi de la nature et de la société :

«Si d'une part la lutte pour la vie a joué un certain rôle dans le progrès matériel des êtres vivants, d'autre part elle a contribué à ce processus seulement dans le détail des formes organiques. Une plus large coordination de tous les grands faits de la biologie nous permet, en revanche, d'établir que les grands types du règne animal se sont constitués dans la paix, grâce aux efforts constants des animaux sur eux-mêmes, grâce à la tension constante de leurs facultés pour triompher des conditions défavorables dans lesquelles certains étaient condamnés à vivre. ${ }^{29}$

Comment s'étonner qu'aient prospéré tant de formes de darwinisme social là où la concurrence, le conflit et même la guerre étaient présentés comme des lois bénéfiques de la nature, et autant d'antidarwinisme social là où on évoquait l'importance décisive de la coopération ? ${ }^{30}$ Comment s'étonner des dits «abus» du darwinisme de la part de philosophes, de politiciens, de sociologues et de littéraires, si même des savants qui faisaient autorité, darwiniens et antidarwiniens n'avaient pas compris?

La question est: pourquoi n'ont-ils pas compris? Les raisons sont nombreuses et diverses: le poids de certaines traditions disciplinaires, de paradigmes et styles de recherche incompatibles, de présupposés et conditionnements philosophiques ou idéologiques, d'intérêts, la tentative de ramener l'image darwinienne de la nature à une image pré-darwinienne plus rassurante, le désir de trouver dans la nature une sanction de ses opinions morales et politiques... Demeure le fait que des spécialistes de telle ou telle discipline scientifique ont mal compris, quand ils n'ont pas carrément déformé. Mais peut-être ne peut-on pas prétendre que ceux qui ont d'autres choses à faire pratiquent de subtiles analyses conceptuelles avant d'utiliser un terme.

\section{Déconstruire la sélection}

Comme la «lutte pour l'existence », la «sélection naturelle» (terme que Darwin avait forgé par analogie avec la "sélection artificielle" pratiquée par les éleveurs et les jardiniers) fut l'objet de déconstructions, intégrations, reformulations. Cette activité commença dès la traduction de l'Origin. Le géologue Bronn, qui en fut le premier traducteur allemand, avait d'abord pensé rendre natural selection par un bizarre Wahl der Lebensweise ("choix du mode de vie»). On le persuada de se replier sur natürliche Züchtung ${ }^{31}$. En allemand furent utilisés ensuite natürliche Zuchtwahl, natürliche Auslese, natürliche Auswahl, mais aussi Naturauslese et Naturzüchtung (et il y eut même des personnes pour faire de savantes distinctions entre ses deux dernières, en critiquant évidemment Darwin pour avoir confondu deux processus distincts ${ }^{32}$ ). Haeckel, qui éprouvait $\mathrm{du}$ plaisir chaque fois qu'il forgeait un néologisme, était même capable d'utiliser trois variantes dans la même ligne: Diese Auslese der Besten, $d$. $h$. die Auswahl der am meisten Begünstigsten zur Naturzucht... (Traduction approximative : «... cette sélection

28. Ibid., p. $88,89,91$

29. E. Perrier, France et Allemagne, p. 103.

30. A. La Vergata, Colpa di Darwin?, p. 73-94

31. G. Wilson Leonard (dir.), Sir Charles Lyell's Scientific Journals on the Species Question, p. 353. Qui sait ce qu'aurait imaginé Bronn pour traduire kin selection?

32. G. Seidlitz, Die Darwinsche Theorie, p. 80, 199. 
des meilleurs, autrement dit le tri des plus favorisés par le choix de la nature... ${ }^{33}$. Ce n'est que bien longtemps après que Selektion s'est imposée ${ }^{34}$.

Inévitablement, les reformulations de «sélection naturelle » se mêlèrent à celles de « lutte pour l'existence ». Le résultat fut une prolifération de luttes et de sélections, tissées et détissées de manière variable. À la sélection naturelle s'ajouta (ou naquit d'une de ses côtes) une "sélection sociale", qui à son tour engendra une "sélection économique ", une "sélection matrimoniale », une " sélection institutionnelle», qui, à son tour, pouvait être «ecclésiastique», « militaire», « sanitaire», " pénale»... Certains distinguèrent entre une "sélection létale » et une "sélection reproductive ». D'autres proposèrent une distinction encore plus articulée: "sélection autogénérique », "hétérogénérique ", « inorganique». La "sélection reproductive», ou "génétique», ne pouvait être contrastée que par une sélection plus forte - la sélection naturelle -, qui pouvait prendre la forme de "sélection périodique » ou de « sélection séculaire ». Mais voilà que défilent devant nous aussi la survival of the unlike, la natural elimination, la constitutionelle Auslese et la dotationelle Auslese, à ne pas confondre avec la balanced e unbalanced selection, la comparative et superlative selection, l'environmental selection, l'active et passive selection, la dominational selection... 35

What's in a metaphor! La volonté de mettre de l'ordre naît du même désir qui engendre le désordre. Inévitablement, quiconque pouvait proposer sa déconstruction, tant il est vrai que la confusion naissait justement de l'abondance des propositions! On en est arrivé à soutenir qu'une des causes principales du darwinisme social classiste, raciste et va-t-enguerre en Allemagne fut la traduction déviante du struggle for life par Kampf ums Dasein plutôt que par le moins belliqueux Anstrengung um den Lebensunterhalt ${ }^{36}$. Combien de délires sanguinaires auraient épargné une bonne traduction! Puissance des mots! (et des exorcismes a posteriori)!

\section{Quelques considérations générales}

Que penser de cette prolifération de termes et de concepts? Excès professoraloacadémico-spécialistico-narcissique? Aussi. Confusion? Bien sûr, mais confusion créative, si exaspérante qu'elle soit pour l'historien : toute cette ébullition était le signe qu'il se passait quelque chose d'important. Les idées véhiculées par les métaphores darwiniennes avaient déchaîné des processus irréversibles, incontrôlables, aux conséquences imprévisibles. Les idées ne sont pas des entités fixes. Elles subissent des modifications, qui peuvent être des adaptations, des traductions plus ou moins fidèles. L'infidélité de la traduction peut se révéler être un facteur de succès. Le mouvement des idées est mouvement précisément parce qu'il comporte des réinterprétations, des hybridations, des contaminations et des malentendus.

Essayer de «démétaphoriser » les métaphores est inévitable, du moins autant que la tendance à se servir de métaphores: l'ambiguïté peut être suggestive et même créative, mais en général il vaut mieux ne pas courir de risque, d'autant plus que, tout comme les mots, les métaphores se banalisent, se congèlent, elles prennent un certain sens comme par inertie (ou par mort des alternatives : pas nécessairement une survie du plus apte), et à la fin on en oublie qu'elles ont été des métaphores, et leur sens littéral n'en est plus clair que pour les philologues (ou pour le philosophe heideggérien, qui, le "re-découvrant », croit penser " pro-fond»). Les métaphores, en somme, finissent pas perdre, comme disent les connaisseurs, leur «prégnance» (autre métaphore!). Du reste, il est fatal qu'une pensée profonde perde de sa profondeur quand ses résultats pénètrent dans les esprits de

33. E. Haeckel, Generelle Morphologie der Organismen, vol. II, p. 232.

34. En français, dans un premier temps, on a eu aussi élection naturelle ; ainsi qu'en italien: elezione naturale et scelta naturale. En espagnol selección natural dès le début. En français survival of the fittest devint survivance des mieux adaptés et survie des plus aptes.

35. Qu'il me soit permis de renvoyer encore une fois à A. La Vegata, L'equilibrio e la guerra della natura, p. 487503.

36. Gottschalk R., «Darwin und der Sozialdarwinismus». 
ceux qui en bénéficient sans l'avoir créée. D'un cerveau à l'autre passent des signes, et quelquefois des idées, pas des mondes spirituels. Cela se produit dans la science comme dans d'autres milieux: "surmoi », " main invisible», «lutte de classe », "équilibres ponctués», «égoïsme génétique », «empreinte», "société civile», «hystérie »... des légions de mots qui ont derrière eux des pensées et des réflexions, des efforts de conceptualisation et des constructions intellectuelles ont perdu, ou presque, tout lien avec leur origine; on les écoute sur la bouche de personnes qui n'ont jamais lu une ligne de Freud, Smith, Hegel, Marx, Dawkins, Lorenz... De la même manière, on peut parier que nombre de biologistes utilisent à bon escient «sélection naturelle » sans jamais avoir lu un texte de Darwin : ce n'est tout simplement pas une exigence de leur profession. Nous demandons au médecin de soigner la maladie de Parkinson, pas de savoir ce que Parkinson pensait du rapport entre maladie et santé. "Influenza » est devenu un terme technique même pour les médecins (non francophones) qui ne croient pas aux influences des astres. Vice-versa, des termes absolument, prosaïquement techniques deviennent des métaphores à usage multiple; il suffit de penser à l'ADN et à toutes les choses qu'on lui attribue dans les discours de chaque jour: les talents d'un footballeur, la ruse d'un homme politique, même les habitudes de toute une nation.

Chaque pensée humaine, en sortant du creuset de l'esprit, perd son lien vital avec le monde intérieur où elle est née; elle peut se refroidir, se cristalliser, se fragmenter, mourir, ou bien survivre en se transformant et en recueillant dans le temps des sens différents, à la manière d'un chalut. Elle peut acquérir et perdre en même temps. Son voyage la portera dans les endroits les plus inimaginables, pour disparaître peut-être dans une petite gare perdue ; peut-être elle finira par signifier toute autre chose. C'est le cas, par exemple, du verbe "dégénérer », qui, né comme terme neutre (« différent de son parent»), a acquis un sens péjoratif. Des considérations semblables devraient nous vacciner pour toujours contre cette maladie que je voudrais appeler "sophisme de l'authentique », et qui consiste à croire que le sens véritable, essentiel, soit celui d'origine, révélé par l'étymologie. Cette maladie frappe surtout quelques philosophes, et en particulier les habitants du «Heideggerland».

Et pourtant, le monde intérieur d'un savant n'est jamais seulement privé. Même celui qui écrit une note de laboratoire ou une impression fugitive sur son journal a devant lui un public, un lecteur possible. Si ce n'était pas le cas pourquoi noterait-il cela d'une certaine manière ou dans un certain style?

L'usage de méthodes quantitatives et mathématiques garantit-il en soi des ambiguïtés ? Les mathématiques tuent-elles les métaphores? Ou mieux, vu que le langage mathématique lui-même grouille de métaphores (bien que tellement vieilles et pétrifiées qu'elles sont devenues inoffensives: bien sûr, même l'étudiant le plus ingénu ne se méprend pas sur le sens du mot «sein » en trigonométrie...), les métaphores sont-elles tuées par les nombres? La mathématisation de la biologie évolutionniste semble avoir progressivement clarifié et, pour ainsi dire, "démétaphorisé » et " purgé de leurs aspects théâtraux » jusqu'aux métaphores de la lutte et de la sélection ${ }^{37}$. On en est arrivé au point presque paradoxal qu'on utilise le terme «fitness darwinienne» pour éviter les équivoques, et pour indiquer quelque chose que Darwin n'a jamais dit avec autant de précision $^{38}$.

37. Y. Conry, «Organisme et organisation : de Darwin à la génétique des populations», p. $292,319$.

38. Il est en effet entré dans la génétique des populations avec R.S. Fisher, qui en a fait un paramètre démographique statistique qui décrit le taux d'augmentation d'une population ou d'un organisme donné dans une population. L'expression « fitness darwinienne » est particulièrement efficace en français ou en italien, grâce à l'éloignement du terme anglais de la langue quotidienne. En anglais, l'effet est mineur, et provient surtout de $l^{\prime}$ adjectif, puisque pour un anglophone fitness est un mot du langage courant, et ne provoque donc pas l'effet d'éloignement nécessaire à un terme technique. Naturellement, tant en anglais qu'en français ou en italien, l'ajout de l'adjectif "darwinienne » empêche que fitness soit entendu dans le sens de la qualité qui s'acquiert avec une vie saine et en consommant certains produits ou en s'entraînant dans une salle de gym... Une équivoque semblable, nous l'avons vu, à celle où sont tombés quelques « déconstructeurs " scientifiques de la sélection naturelle. 
La biologie postdarwinienne a introduit d'autres métaphores : "flux génique », "dérive génétique », "programme», «code», «bricolage», "ADN poubelle» (junk DNA), " gènes modificateurs et gènes régulateurs", "information ", "transcription ", "saut évolutif », ... et, dans les applications de la théorie des jeux, "faucons », " colombes», «poulets » (dans le sens d' 'innocents »), et même " bourgeois opportunistes ». Sont-elles aussi partie intégrante de la pensée évolutionniste, ou bien, vu qu'aujourd'hui «nous en savons plus » et que nous avons des instruments plus "objectifs », sont-elles réservées à la vulgarisation? L'idéal de l'objectivité scientifique semble exiger que les métaphores soient «mises hors d'état de nuire ». Cela est-il possible ? Peut-être sommes-nous au milieu d'une lutte pour l'existence entre les mots et les nombres. Qu'en sera-t-il des choses?

\begin{abstract}
Résumé
On pense généralement que les métaphores ne figurent dans le discours scientifique qu'à titre d'exemples, de suggestions rhétoriques ou d'images favorisant la représentation intuitive de concepts difficiles; bref, elles relèveraient plutôt du domaine de la stratégie communicative et de la vulgarisation que de celui de la construction des théories. Cela n'est vrai que partiellement: l'histoire des sciences nous présente beaucoup de cas où les métaphores ont aidé le savant lui-même à penser sa théorie, ou, autrement dit, elles ont rempli une fonction autodidactique aussi bien que didactique, ainsi que le montre l'analyse d'un cas très important, celui des métaphores darwiniennes de «lutte pour la vie » et de «sélection naturelle». Elles ont engendré des méprises non seulement chez les adversaires mais aussi chez bien des défenseurs de Darwin. La diffusion n'a donc pas coïncidé avec la compréhension. C'est paradoxal, mais c'est normal dans l'histoire des idées.
\end{abstract}

\title{
Bibliographie
}

BERNARDINI Jean-Marc, Le darwinisme social en France (1859-1918). Fascination et rejet d'une idéologie, Paris, CNRS Éditions, 1997.

CONRY Yvette, "Organisme et organisation : de Darwin à la génétique des populations », Revue de Synthèse, III ${ }^{\mathrm{e}}$ série, vol. CII, n 103-104, p. 291-321.

DARWIN Charles, L'origine des espèces par le moyen de la sélection naturelle, ou la préservation des races favorisées dans la lutte pour la vie. Édition du Bicentenaire sous la direction de Patrick TORT, traduction par Aurélien BERRA, coordination par Michel PRUM, Paris, Champion (Champion Classiques), 2009.

Darwin Charles, Notebook D, dans BARretT Paul H., GaUtrey Peter J., Herbert Sandra, KoHN David et SMITH, Sydney (dir.), Charles Darwin's Notebooks, 1836-1844, Londres, British Museum (Natural History) - Cambridge, Cambridge University Press, 1987.

Delage Yves, L'hérédité et les grands problèmes de la biologie (1894), 2e éd., Paris, Schleicher Frères, 1903.

DRUMMOND Henry, Tropical Africa, Londres, Hodder and Stoughton, 1888. 
DRUMMOND Henry, Natural Law in the Spiritual World (1883), $2^{\mathrm{e}}$ éd., Londres, Hodder and Stoughton, 1892.

FISKE John, Outlines of Cosmic Philosophy, Based on the Doctrine of Evolution, with Criticisms on the Positive Philosophy, London, Macmillan, 1874.

Flourens Pierre, Examen du livre de M. Darwin sur l'origine des espèces, Paris, Garnier Frères, 1864.

GAUDRY Albert, Essai de paléontologie philosophique, Paris, Masson, 1896.

GotTSCHALK R., «Darwin und der Sozialdarwinismus », Deutsche Zeitschrift für Philosophie, vol. VII, p. 521-539.

GRAY J.-L. (dir.), Letters of Asa Gray, London, Macmillan, 1893, 2 vol.

GRAY Asa, Darwiniana. Essays and Reviews Pertaining to Darwinism (1876), éd. par A. Hunter DupreE, Cambridge, Mass., The Belknap Press of Harvard University Press, 1963.

HAECKEL Ernst, Generelle Morphologie der Organismen, Berlin, Reimer, 1866, 2 vol.

HuXley Thomas Henry, "The Struggle for Existence in Human Society » (1888), dans HuXley Thomas Henry, Collected Essays, Londres, Macmillan, 1893-1894, vol. IX, p. 195236.

HuXleY Thomas Henry, «Evolution and Ethics » (1893), dans HuXLEY Thomas Henry, Collected Essays, Londres, Macmillan, 1893-1894, vol. IX, p. 46-116.

KROPOTKINE Pierre (KROPOTKIN Pëtr Alekseevič), L'entr'aide. Un facteur de l'évolution, traduit de l'anglais sur l'édition revue et augmentée par L. Bréal, Paris, Hachette, 1904.

LANESSAN Jean-Louis, La lutte pour l'existence et l'évolution des sociétés, Paris, Alcan, 1903.

LA VERGATA Antonello, L'equilibrio e la guerra della natura. Dalla teologia naturale al darwinismo, Naples, Morano, 1990.

LA VERGATA Antonello, "Lamarckisme et solidarité », dans BLANCKAERT Claude, COHEN Claudine, CORSI Pietro et FISCHER Jean-Louis (dir.), Le Muséum au premier siècle de son histoire, Paris, Muséum National d'Histoire Naturelle, 1996, p. 313-327.

LA VERGATA Antonello, «Evoluzionismo alla francese contro darwinismo alla tedesca », Paradigmi, vol. XXXIX, n²2, août 2011, p. 67-87.

LA VERGATA Antonello, «La sacculina e il «Regno dell'uomo ». Edwin Ray Lankester, la degenerazione e il futuro della civiltà », Medicina \& Storia, vol. 1-2 (n.s.), 2012, p. 59-83.

LE DANTEC Félix, La lutte universelle, Paris, Flammarion, 1906.

LE DANTEC Félix, L'égoïsme seul base de toute société. Étude des déformations résultant de la vie en commun, Paris, Flammarion, 1911.

LE DANTEC Félix, Savoir! Considérations sur la méthode scientifique, la guerre et la morale, Paris, Flammarion, 1917.

MANIER Eduard, The Young Darwin and His Cultural Circle. A Study of the Influences Which Helped Shape the Language and Logic of the First Draft of the Theory of Natural Selection, Dordrecht-Boston, Reidel, 1978. 
PERRIER Edmond, France et Allemagne, Paris, Payot, 1915.

PIAGET Jean, « La biologie et la guerre », Feuille centrale de la Société suisse de Zofingue, vol. LVIII, 1918, nº 5 , p. 374-380.

SABATIER Armand, Philosophie de l'effort. Essais philosophiques d'un naturaliste, Paris, Alcan, 1903.

SEIDLITZ Georg, Die Darwinsche Theorie. Elf Vorlesungen über die Entstehung der Thiere und Pflanzen durch Naturzüchtung, Dorpat, Matthiessen, 1871.

THOMSON John Arthur, Darwinism and Human Life. The South African Lectures for 1909, Londres, Melrose, 1909.

WILSON Leonard G. (dir.), Sir Charles Lyell's Scientific Journals on the Species Question, New Haven, Yale University Press, 1970. 


\title{
Pourquoi l'anglais est-il la langue de l'économie?
}

\author{
Jean MATOUK \\ Professeur des universités honoraire en économie, \\ Université de Montpellier \\ Extrait de : Xavier LAFON, Les langues savantes, Paris, \\ Édition électronique du CTHS (Actes des congrès des sociétés historiques et scientifiques), 2017. \\ Cet article a été validé par le comité de lecture des Éditions du CTHS dans le cadre de la publication
des actes du $139^{\text {e }}$ Congrès national des sociétés historiques et scientifiques tenu à Nîmes en 2014.
}

De multiples autres disciplines utilisent l'anglais. Presque de force! Car pour être retenu pour une promotion universitaire, dans un colloque, les revues dans lesquelles il faut publier sont à $80 \%$ anglaises ou américaines.

Mais, dans le cas de l'économie, l'anglais est la langue maternelle, une langue dont la domination économique mondiale anglaise au XIX siècle a assuré l'impérialisme linguistique dans les affaires et donc dans la discipline qui les étudie. S'ajoute à ces raisons historiques de la symbiose étroite entre l'anglais et l'économie, une raison pratique, la plasticité linguistique de l'anglais, particulièrement utile en finances.

\section{L'anglais, langue maternelle de l'économie}

Ce sont des auteurs anglais qui ont énoncé les premières vraies lois économiques, les économistes français se contentant, jusqu'à la Seconde Guerre mondiale, au moins, d'importer et de traduire. Pourquoi ? Tout simplement en raison de la précocité économique britannique. Sous Louis XIV, la France domine politiquement ce qui est alors le monde occidental, c'est-à-dire l'Europe. Pourtant, malgré les efforts de Richelieu, puis ceux de Colbert, d'abord dans la marine, puis avec l'industrie - le colbertisme incarne la matrice du développement économique français centré sur l'État -, la Grande-Bretagne, bien que politiquement dominée, commence à prendre de l'avance sur le plan industriel. Elle maintient cette avance durant tout le $\mathrm{XVIII}{ }^{\mathrm{e}}$ siècle tant sur le plan du commerce international, avec la fameuse Compagnie des Indes, que sur le plan financier.

En 1763, le Traité de Paris met fin à la Guerre de Sept ans et marque la fin du primat politique français, le début d'un déclin que Napoléon ne parviendra pas à enrayer. Mais, en même temps, par ce traité, les plus importantes de nos colonies d'alors, notamment l'actuel Canada, passent sous contrôle anglais, comme la petite partie de l'Inde de Dupleix. L'industrie anglaise a évidemment bénéficié de cette victoire dans le commerce international, malgré le coût de l'indépendance américaine, et c'est là, en GrandeBretagne, qu'apparurent les premiers vrais discours sur l'économie moderne.

Pour être juste, on avait eu, auparavant, quelques textes français traitant de faits économiques. Au $\mathrm{XVI}^{\mathrm{e}}$ siècle, Jean Bodin, juriste, attribua l'envolée des prix à l'arrivée massive d'or et d'argent du nouveau monde dans un débat resté célèbre, la Controverse avec Monsieur de Malestroit. C'était la première forme de la fameuse théorie quantitative de la monnaie. À l'extrême fin du XVII ${ }^{\mathrm{e}}$ siècle, Vauban avec sa Dîme royale et de Boisguilbert avec Le factum de la France, attribuèrent à juste titre la faiblesse de notre économie - déjà ! - à la fiscalité, ce qui leur valut d'ailleurs le courroux de Louis XIV. Mais nous n'étions pas dans l'analyse de la production et des échanges, sujet central de 
l'économie, mais dans l'analyse du rôle de l'État dans l'économie, problème toujours d'actualité.

Outre-Manche, dès le XVI ${ }^{\mathrm{e}}$ siècle, durant cette période dite mercantiliste, plusieurs auteurs ont publié des analyses sur les échanges et la monnaie: Thomas Gresham publie sa fameuse loi La mauvaise monnaie chasse la bonne (1579), Thomas Mun publie Discourse of Trade from England unto the east indies (1621) et Josiah Child, directeur de la Compagnie des Indes par ailleurs, publie aussi un New discourse on trade (1668).

William Petty (1623-1687) s'intéresse aussi à la monnaie et surtout à la statistique par le recensement. Il effectue les premières analyses de ce qui deviendra le PIB. De son côté, Gregory King développe la statistique. À la fin du XVIII ${ }^{\mathrm{e}}$ siècle - naissance de l'industrie anglaise - le philosophe Adam Smith, dont le premier ouvrage la Théorie des sentiments moraux (1759) peut être considéré comme le fondateur de l'économie politique moderne. C'est dans un voyage en France, en 1764, notamment à Toulouse et en Provence où, dit-il, il s'ennuie, qu'il commence la rédaction de son Traité. Il ira ensuite à Genève, puis Paris, où il rencontre celui que l'on considère comme le premier économiste français, François Quesnay. Celui-ci, auteur de l'article "Grains » dans l'Encyclopédie, est le fondateur de l'école française qu'on dénomme "physiocrate». Il estime que la seule source de valeur est la terre. L'artisanat, l'industrie, le commerce, ne sont que des consommateurs de la valeur créée par la seule agriculture. Le grand Turgot, tout en étant pour la liberté du commerce, (Lettre sur la liberté du commerce et des grains - 1770), reprend dans Réflexions sur la formation et la distribution des richesses (1776) la thèse de l'agriculture comme seule source de richesse.

Désaccord complet d'Adam Smith, qui de retour à Glasgow, déjà industrielle, écrit son livre fondateur: Recherche sur la nature et les causes de la richesse des nations (1776). Il y décrit l'organisation industrielle, le salariat, les gains de productivité qu'obtient l'industrie par la mécanisation. Il est d'ailleurs pessimiste sur l'avenir et évoque déjà la question du partage des richesses.

Je m'arrête une minute sur le parallèle entyre Quesnay et Smith. L'un vit dans un pays déjà en perte relative de vitesse, la France, où le politique freine plutôt le développement économique, cause de la révolution de 1789 , et où, surtout, pour notre sujet d'aujourd'hui, l'agriculture domine l'économie, ce qui fait que la pensée économique est orientée prioritairement vers l'agriculture. Au contraire, Smith vit en Grande-Bretagne, pays qui est en train de prendre le primat politique et qui est déjà économiquement dominant. La pensée économique anglaise va directement vers l'industrie qui va être le secteur phare du XIX ${ }^{\mathrm{e}}$ puis du XX $\mathrm{X}^{\mathrm{e}}$ siècle, sur lequel va se bâtir le capitalisme triomphant.

$\mathrm{Au} \mathrm{XVII}^{\mathrm{e}}$ siècle, comme au XVIII ${ }^{\mathrm{e}}$ et au XIX, on connaît de brillants mathématiciens, physiciens, chimistes, français. Souvent d'ailleurs - ils le peuvent à l'époque - ils sont pluridisciplinaires. Pascal est physicien, mathématicien et philosophe, comme Descartes. Aussi le français est-il une langue de naissance de ces trois disciplines. Ce n'est pas le cas de l'économie.

En Grande-Bretagne, à la suite d'Adam Smith, David Ricardo (1772-1823), agent de change, écrit des traités sur la monnaie pendant les guerres avec Napoléon, plaide évidemment pour le libre-échange, faisant abolir les corns laws anglaises (lois sur le protectionnisme agricole) pour faire baisser les prix de l'alimentation, ce qui permet de baisser les salaires industriels, et développer les exportations. Finalement, il écrit son livre principal, Des principes de l'économie politique et de l'impôt (1817), qui est à son tour le texte fondateur de ma discipline et qui, lui aussi, d'ailleurs est pessimiste sur la redistribution, voyant la rente amputer les profits, donc les salaires. À côté de lui, Malthus (1766-1834), dont on ne retient en général que son Essai sur le principe de la population (1798), également pessimiste, qui conseille aux prolétaires de modérer leurs transports sexuels. Mais lui aussi a écrit des Principes d'économie politique au point de vue de leur application pratique (1820). Citons aussi Stuart Mill, plus tardif (1816-1876), que nous dirions plus à gauche aussi, qui vécut en France et publia également des Principes en 1848. Enfin, on doit 
considérer que Le Capital de Karl Marx (1867) est une œuvre d'économiste, directement inspirée de l'industrie anglaise que son comparse, Engels, connaissait bien par son père. Bref, au moins cinq fondateurs de ma discipline sont nés ou ont travaillé en GrandeBretagne.

Et en France ? Jean-Baptiste Say, retenant uniquement de Smith et Ricardo la liberté du commerce et la concurrence, importa en France une théorie économique particulièrement appauvrie. Sa fameuse phrase : "l'offre crée sa propre demande» est une erreur. Si toutes les entreprises embauchent, achètent ou louent des machines et achètent des matières premières pour produire une offre, rien ne dit qu'une demande absorbera entièrement cette offre. C'est pourtant à cette théorie que s'accrocheront encore ceux $q^{\prime}$ 'on appelle les « néoclassiques » français de la fin du XIX ${ }^{\mathrm{e}}$ siècle.

Quand ces théories néoclassiques, qu'on appellerait, aujourd'hui, d'ailleurs à tort, « ultralibérales », feront flores en Grande-Bretagne même, c'est encore d'un économiste anglais, John Meynard Keynes, que viendra la réfutation décisive en 1936... laquelle sera traduite en français en 1963 seulement, par l'inspecteur des finances Jean de Largentaye. Bref, notre discipline est anglaise de naissance, et à ce premier titre historique, il est normal que sa langue soit l'anglais. Mais, si « l'anglicité » de l'économie tient d'abord au fait que les premiers vrais économistes étaient anglais, elle tient aussi à la domination britannique totale sur l'économie mondiale de 1815 à 1913.

Le XIX ${ }^{e}$, c'est le siècle de la Grande-Bretagne. La City domine la finance mondiale ; elle est en liaison avec les banques que souvent elle a créées sur tous les continents, au sein du Commonwealth (dont la traduction littérale est "richesse commune », ce qui souligne encore le primat de l'économie). Londres est le premier port du monde et la GrandeBretagne la première exportatrice et importatrice. Tous les contrats financiers ou commerciaux sont rédigés dans la langue de Shakespeare. Un autre détail : les Anglais sont aussi les premiers touristes internationaux et vont longtemps le rester.

Dans le seconde partie du au XIX ${ }^{\mathrm{e}}$ siècle, lors de la «mêlée pour l'Afrique ${ }^{1}$ » et de la colonisation de l'Asie, la France va aussi avoir sa part. Mais, pour l'Afrique par exemple, à y regarder de près, en l'Afrique du nord et au Sahel, les colonies françaises ont fort peu des matières premières qui sont à la base de la croissance industrielle du XIX $\mathrm{X}^{\mathrm{e}}$ - le pétrole ne sera exploité en Afrique de l'ouest qu'à partir du dernier tiers du XX $X^{\mathrm{e}}$-alors que les colonies anglaises recèlent non seulement d'or et de diamants, mais aussi toutes les matières premières dont a besoin le capitalisme pour sa deuxième révolution industrielle. L'intensité commerciale au sein du Commonwealth, avec les pays d'Asie, a été infiniment plus grande qu'entre les colonies françaises et leur métropole. La langue anglaise s'impose aussi dans la plus grande partie du monde comme langue du commerce et de l'économie.

J'en viens à la troisième raison qui fait de l'anglais la langue de l'économie : sa plasticité linguistique. C'est vrai dans de nombreux domaines. Les Anglais sont, je crois, plus inventifs que nous pour dire en une ou deux syllabes quelque chose de compliqué qui devient simple et courant. Courriel n'a pas pris, c'est mél ou mail qui triomphe! Vous entendrez bien peu d'économistes français utilisez le mot "mercatique », que je ne sais plus quel gouvernement voulait imposer pour franciser marketing, qui comporte une syllabe de moins, mais qui surtout, au participe présent, signifie immédiatement «agir sur le marché ». Merchandising aussi! Mais c'est encore plus évident en matière financière. Comment croyez-vous que les financiers parlent d'un contrat d'échange de taux d'intérêt ou d'un contrat d'échange de taux de change, déjà pas facile à expliciter en français? Ils vont dire interest swap ou change swap? Et tout le monde - le monde financier, je veux dire, et même un peu plus - comprend. Parleront-ils d'un " contrat d'échange d'assurance crédit »? Mais non ! CDS - Credit default swap-, dont tout le monde

1. Expression tirée de l'anglais «The Scramble for Africa », d'après l'ouvrage éponyme de Thomas Pakenham, relatif au partage du continent entre les différentes puissances européennes. 
économique, connaît les initiales. Future au lieu de «contrat de futur » qui n'est d'ailleurs pas correct en français. Cap ou floor pour un «contrat de plafonnement» ou «de plancher » Call pour une « option d'achat » et put pour une «option de vente »

La langue anglaise est la langue idéale par cette plasticité pour les opérations financières qui se déroulent en quelques minutes. Bref, si vous me permettez cette comparaison grossière trop souvent entendue, si le français est la langue de la raison ou de la littérature, l'espagnol celle qu'il faut utiliser pour parler à Dieu (quand on a oublié le latin...), l'allemand suivant l'estime qu'on a pour nos amis teutons, celle qu'on utilise pour philosopher, ou... qu'utilisent les dresseurs pour parler aux animaux de cirque, l'italien pour parler aux femmes, l'anglais est assurément la langue du business... pardon... du commerce et des affaires.

\section{Résumé}

Plusieurs raisons expliquent que l'économie ait une langue spéciale qui est l'anglais. L'économie politique, comme discipline, est née en Grande Bretagne, à la fin du XVIII ${ }^{\mathrm{e}}$, avec Adam Smith, autour de l'industrie anglaise naissante, tandis que les physiocrates français ne voient de «richesse » que dans l'agriculture. Le développement économique de la GrandeBretagne, "son » XIX ${ }^{\mathrm{e}}$ siècle - activité commerciale et bancaire internationale -, ont largement alimenté des études économiques, la plupart du temps, anglaises. Des économistes français "suivaient " mais ne générèrent aucune école spécifique, à l'exception du nîmois Charles Gide. La traduction en français de la Théorie générale de John M. Keynes après 1936 fut un moment refondateur de l'économie politique en France.

Enfin la troisième raison pour laquelle l'anglais est la langue de l'économie est ce que l'on pourrait appeler sa plasticité terminologique. Un mot commun (swap, future, cap, flour) devient très vite un terme technique précis, adopté par tous les professionnels, alors que les mots français correspondants ne gardent que leur sens premier. Tous les financiers connaissent et utilisent le sigle CDS (Credit Default Swap) dont la traduction française serait « inutilisable».

Mais, outre cette «nationalité » d'origine anglaise, la langue de l'économie a une autre caractéristique linguistique, lexicale. C'est l'usage fréquent sinon permanent du pourcentage, à l'image de la date en histoire. Un texte économique est obligatoirement scandé de pourcentages. Peu importe en effet de connaître le niveau absolu du produit national brut total ou par tête, ce qui caractérise un état de tout ou partie de l'économie, c'est l'évolution de ce PNB, de cette consommation, des prix, des salaires donc du pouvoir d'achat, en pourcentage; ou c'est le taux de chômage, pourcentage des chômeurs par rapport à la population active totale. La sociologie, la géographie utilisent aussi les pourcentages, mais dans une moindre mesure. 


\title{
Professionnels et professionnelles de la publicité française : les femmes dans l'histoire de la profession publicitaire de 1925 à nos jours
}

\author{
Nathalie PELIER \\ Docteure en histoire sociale des professions \\ Extrait de : Xavier LAFON, Les langues savantes, Paris, \\ Édition électronique du CTHS (Actes des congrès des sociétés historiques et scientifiques), 2017. \\ Cet article a été validé par le comité de lecture des Éditions du CTHS dans le cadre de la publication \\ des actes du $139^{\mathrm{e}}$ Congrès national des sociétés historiques et scientifiques tenu à Nîmes en 2014.
}

Pourquoi l'historien des professions est-il amené à adopter une approche « genrée » des professionnels qu'il étudie? Cette orientation est liée à un contexte général : depuis quelques années, et sous l'impulsion de l'historiographie anglophone notamment, un certain nombre de colloques intégrant une posture "sexuée» ont vu le jour dans l'Hexagone : "Genre et technique » (Besançon, 2012), "La vocation au féminin » (Lille, juin 2014) ou encore "Inégalités hommes-femmes et utopies» (Lille, juin 2015). Le législateur se préoccupe d'autre part de plus en plus d'inciter chaque employeur à mettre en place une plus forte équité entre ses collaborateurs des deux sexes sous peine de sanctions $^{1}$, mais aussi à travers quelques aides financières et techniques ${ }^{2}$. Les historiens du travail et des professions sont donc de plus en plus amenés à adopter une posture " genrée » parce que les milieux professionnels eux-mêmes sont tenus (théoriquement du moins) de se préoccuper d'une meilleure équité en matière d'accessibilité aux métiers et d'évolution professionnelle. Certaines professions se prêtent plus ou moins facilement à une approche par le genre. L'histoire de la profession publicitaire se montre assez récalcitrante à cela. Nous allons en voir les raisons et évoquer quelques figures féminines de la profession publicitaire française.

\section{L'État de la recherche : le problème des sources et archives}

En France, l'histoire des «publicitaires » intéresse depuis très peu de temps, à la suite des études historiques (peu nombreuses elles aussi ${ }^{3}$ ) sur la publicité en tant que telle. MarieEmmanuelle Chessel, auteure de Publicité, naissance d'une profession (1900-1940) publié en 1998, s'est beaucoup appuyée notamment sur les travaux de l'historien des médias Marc Martin et son incontournable Trois siècles de publicité en France (1992) qui, comme le titre l'indique, traite davantage la publicité que ses producteurs. Son ouvrage Les pionniers de

1. Écarter par exemple une personne d'une procédure de recrutement en raison de son sexe expose à des sanctions prévues par l'article 225-2 du Code Pénal ( 3 ans de prison et 45000 euros d'amende).

2. Le «contrat d'égalité professionnelle», dont toutes les entreprises et branches professionnelles peuvent bénéficier, a par exemple pour objet le financement d'actions mises en œuvre pour l'égalité en matière d'embauche, d'évolution professionnelle et d'aménagement des postes de travail. $50 \%$ des coûts d'investissement en matériel et $30 \%$ de ceux en matière de rémunération pendant un temps de formation par exemple peuvent être pris en charge par la collectivité !

3. Dans le champ des études littéraires, la publicité (affiche mise à part en raison d'une proximité avec l'histoire, " noble ", de la peinture) est longtemps passée pour quelque chose de vulgaire. Toutefois, ces dernières années, de nombreuses manifestations scientifiques ont été organisées, comme, par exemple, le colloque «Littérature et publicité : de Balzac à Beigbeder » (Union Centrale des Arts Décoratifs, Paris, avril 2011). 
la publicité, aventures et aventuriers de la publicité en France (1836-1939) est par ailleurs assez récent puisqu'il ne date que de 2012! L'omniprésence de la publicité, son caractère envahissant, peuvent donner l'impression que dresser l'histoire des publicitaires est chose aisée. Contrairement à ce que l'on pourrait croire, il n'en est rien. Les archives, matériau de base de l'historien, sont, en ce qui concerne les producteurs de la publicité, encore plus que pour la publicité en tant que telle, difficilement accessibles et fort peu nombreuses. À juste titre, l'historienne de la publicité Véronique Pouillard a observé :

«[...] Les publicitaires apparaissent comme un groupe sociologique porté vers les campagnes à venir et rejetant ce qui, vieilli [...], ne peut qu'encombrer leur marche vers la nouveauté. [...] La conservation de nombreux et volumineux documents requiert une infrastructure et des choix propres à décourager les volontés. De plus, la confidentialité des affaires traitées, qui implique plusieurs parties et des budgets parfois importants [...] a instauré dans les milieux publicitaires, dès le milieu du XIX ${ }^{\mathrm{e}}$ siècle, une pratique $\mathrm{du}$ secret $[\ldots] »^{4}$

En dépit de la présence plus ou moins forte de certains professionnels de la pub dans les médias (Jacques Séguéla par exemple côté hommes ou Mercedes Erra, sur laquelle nous allons revenir, côté femmes), on sait donc peu de chose sur les publicitaires de façon générale et encore moins sur les femmes publicitaires en particulier. Cela est a priori tout à fait surprenant: si les cadres de la publicité sont des hommes à $72 \%$, les effectifs des agences installées en France sont à $62 \%$ féminins ${ }^{5}$. Le musée de la publicité à Paris possède bien une chemise documentaire constituée de coupures de presse portant sur les femmes publicitaires, mais leur histoire professionnelle n'a pas encore fait l'objet d'une étude scientifique.

\section{Les palliatifs à des archives inexistantes/inaccessibles}

À défaut de pouvoir consulter des archives d'entreprise utiles à l'établissement de l'histoire des «pros de la pub», il reste la possibilité d'exploiter les éventuels ouvrages biographiques et autobiographiques ainsi que la presse professionnelle.

\section{La presse professionnelle}

Contrairement à une idée reçue, le célèbre magazine Stratégies, né au début des années 1970, n'est pas la seule revue française destinée aux professionnels de la publicité. Ces derniers ont constitué une presse spécialisée tournée sur eux-mêmes à une date relativement haute et c'est dans deux périodiques aujourd'hui disparus (La publicité, revue mensuelle des méthodes modernes de vente et Vendre) que l'on trouve les traces les plus anciennes de femmes publicitaires. La première, une émanation de l'agence Hémet, Jep \& Carré, est parue dès 1902 et a été publiée jusque dans les années 1930. Vendre a été éditée à partir de 1923, durant une cinquantaine d'années. Émanation d'une autre célèbre agence de la première moitié $d u X X^{\mathrm{e}}$ siècle, celle des frères Damour, elle a été le portedrapeau de la profession «publicitaire » modernisée, laquelle a voulu laisser derrière elle les «publicistes» pour ne laisser la place, petit à petit, qu'à des «publicitaires », fabricants d'une publicité moralisée ${ }^{6}$, d'une part et, d'autre part, conçue davantage au service des annonceurs (commanditaires des campagnes) que des organes de presse ${ }^{7}$. Il

4. V. Pouillard, La publicité en Belgique 1850-1975, des courtiers aux agences internationales, p. 23.

5. Statistiques 2014 de l'Association des Agences Conseils en Communication.

6. En décembre 1917, Les publicitaires, p. 4, bulletin de la Conférence des Chefs de Publicité, expliquait que le " rôle » des (bons) professionnels de la publicité était d' " aider (les annonceurs) à gagner toujours plus » mais précise par ailleurs qu'ils devaient rester « épris de conscience professionnelle».

Il ne s'agissait pas de faire gagner «plus » par n'importe quel moyen, c'est pourquoi dès 1921, la Chambre Syndicale de la Publicité adoptera son premier Code des usages.

7. Marie-Emmanuelle Chessel a observé qu'au début du XX $X^{\mathrm{e}}$ siècle, « deux définitions de l'activité publicitaire » avaient co-existé. La première, portée aux nues par la Chambre Syndicale de la Publicité (créée en 1906), consistait en un « échange d'espace publicitaire au service d'un organe de presse ». Dans la seconde conception, celle de la Corporation des Techniciens de la Publicité (née en 1920, mais en gestation dès 1913 au sein de la 
s'agit donc là de deux périodiques très importants pour l'historien de la publicité et de ses praticiens.

Le centre de documentation du musée de la publicité à Paris a procédé au très précieux dépouillement de Vendre en 2006-2007 et on dispose de la quasi-totalité des numéros de la revue. En revanche, de nombreux «trous» perturbent l'état de la collection de $\mathrm{La}$ publicité... mais une lecture de l'ensemble des sommaires de Vendre et l'examen de chaque exemplaire de La publicité... permettent d'établir que tous les collaborateurs de ces périodiques étaient des hommes, à l'exception d'une certaine Paule de Gironde, auteure, entre 1926 et 1931, de treize articles publiés dans Vendre, et de Jeanne Laforest, signataire d'une vingtaine de compositions rédigées pour La publicité... entre 1931 et 1939. Qui étaient ces deux femmes ? Les articles de la première se terminent volontiers avec la reproduction d'une signature manuscrite à laquelle s'ajoute la mention " technicien en publicité » (et non pas " technicienne » comme nous serions tentée de le dire aujourd'hui). Quant à la seconde, elle s'est elle-même définie comme «publicitaire » dans un article publié en octobre $1937^{\circ}$. À l'heure actuelle, nous ne savons encore strictement rien sur leur vie personnelle.

Les travaux écrits de ces deux femmes, peut-être les deux premières publicitaires françaises, sont essentiellement constitués de conseils pratiques et d'analyses sur des travaux réalisés par des confrères dont elles ne précisent toutefois pas le nom. Elles n'ont laissé strictement aucun indice concernant leur propre production publicitaire. Le fait que cette dernière soit totalement inconnue à ce jour du moins n'est pas lié à leur sexe : entre 1938 et 1970, le rédacteur Pierre Herbin (1900-1981), notamment collaborateur d'Havas dans les années 1960, a écrit une douzaine de livres portant sur la rédaction publicitaire ou la publicité et son environnement de façon générale, mais il est impossible, pour le moment, de préciser à quelles campagnes il a collaboré. Constituer un énorme corpus de publicités en espérant repérer une création de Paule de Gironde, Jeanne Laforest ou même Pierre Herbin serait vain : contrairement aux œuvres littéraires et artistiques qui supportent très bien la précision d'un nom d'auteur (même inventé de toutes pièces), celles de la publicité (mis à part les deux cas particuliers des « réclames ${ }^{9}$ » du XIX siècle et des affiches signées par des peintres) gardent pour elles le nom de leurs géniteurs/génitrices. Le grand public n'est en fait informé de l'identité des créateurs d'une publicité que dans un contexte limité ${ }^{10}$ (par exemple, expositions du musée de la publicité, soucieux de proposer une légende nominative).

Les textes de Paule de Gironde ont une facture extrêmement pragmatique : destinés à parfaire la technique des lecteurs, ils sont riches d'éléments susceptibles de renseigner l'historien sur les pratiques professionnelles publicitaires de la seconde moitié des années 1920 à la première des années 1930. « Mettez-vous dans la peau de l'acheteur ! [...] [Les annonces] sont faites trop souvent par des gens qui pensent ou qu'on oblige à penser comme des annonceurs", exhortait l'auteur en 1927 dans son article "Si nous faisions une annonce : comment la construire ${ }^{11}$ ? ». Celui-ci nous apprend que pour être efficace, la personne qui souhaite composer une annonce avec professionnalisme doit se mettre à la place du client final (le consommateur) et non pas à la place du client du publicitaire, l'annonceur. Par déduction, on peut supposer qu'une erreur potentielle des rédacteurs du premier tiers du XX $X^{\mathrm{e}}$ siècle a consisté à écrire en se trompant de cible. Paule de Gironde,

Conférence des Chefs de Publicité), l'activité publicitaire était plutôt « une technique au service de l'annonceur ». M-E. Chessel, Publicité : naissance d'une profession (1900-1940), p. 19.

8. J. Laforest, « La joie fait vivre le commerce», p. 698-701. À la page 698, l'auteur déclare : «[...] Nous sommes là pour $[. .$.$] savoir, nous autres publicitaires ».$

9. Au XIX ${ }^{\mathrm{e}}$ siècle, certaines "réclames » (articles laudatifs insérés dans un journal contre rémunération) ont pu être signées, car les pseudo signatures qui terminaient les textes devaient donner l'illusion d'articles journalistiques. Le nom des signataires était souvent inventé. Dans sa Dernière mode (1874), Stéphane Mallarmé (1842-1898) signait « Miss Satin » sa rubrique Fashion. S'ils donnaient à voir leur véritable identité, les auteurs de ces textes, journalistes ou écrivains, s'exposaient à un fort discrédit.

10. Depuis juin 2009, un moteur de recherche de l'INA permet toutefois à tout un chacun (et non pas seulement aux seuls chercheurs et personnels des agences de publicité) de consulter près de 210000 créations diffusées depuis 1968 !

11. P. (de) Gironde, Vendre, « Si nous faisions une annonce : comment la construire ? », p. 279. 
qui signait ses compositions avec la mention «Technicien en publicité » semble avoir évolué vers une fonction de «cadre ». À la fin des années 1920, elle recrutait le personnel créatif pour l'agence Damour. Elle était l'équivalent actuel d'une «directrice de la création »! Le célèbre homme de lettres Jean Anouilh (1910-1987) a travaillé comme rédacteur au sein de cette entreprise grâce au talent de "chasseuse de têtes » de Paule de Gironde.

Ce qui saute surtout aux yeux dans les articles de Jeanne Laforest, ce sont leurs très nombreuses références à des marques, entreprises et produits étrangers alors que les compositions de Paule de Gironde sont nettement plus «franco-françaises ». La collaboratrice de La publicité... a probablement beaucoup voyagé en Europe. Mais Paule et Jeanne ne sont peut-être, à vrai dire, qu'une seule et même femme! La première a peut-être quitté la revue Vendre et pris un pseudonyme pour s'associer à La publicité [...], périodique d'une agence concurrente. Étrangement en effet, la formulation du titre d'un des articles de Jeanne semble faire écho à celle d'études, antérieures, de Paule. En décembre 1926, Vendre publiait l'une d'elles sous le titre "Si nous faisions une chronique "; en mars 1936, La publicité... donnait à lire "Si nous lancions la blanchisserie MON-PRÉ », sorte d'étude de cas fictif. Les articles "en Si... » semblent avoir été instaurés par Vendre qui a publié, des années 1920 aux années 1930 incluses, toute une série de «papiers » dont le titre commençait par «Si... » et dont tous les auteurs étaient des hommes (sauf Paule de Gironde). On perd par ailleurs la trace de Paule en 1931 et commence à repérer celle de Jeanne la même année. Curieuse coïncidence...

Comme l'a démontré Christine Bard ${ }^{12}$ notamment, le tertiaire se féminise durant l'entredeux-guerres. Il semble tout à fait probable que d'autres femmes que Paule de Gironde et Jeanne Laforest aient travaillé dans une entreprise publicitaire tout en n'ayant pas forcément écrit pour une revue professionnelle. Plutôt que "publicitaires » ${ }^{13}$ en tant que telles, la majorité des femmes ayant peut-être travaillé dans une agence « de pub » devait être dactylographes et secrétaires, métiers " féminins » dit-on. Marcel Bleustein-Blanchet (1906-1996), fondateur de Publicis ( $n^{\circ} 3$ mondial de la pub) a par exemple évoqué dans sa Rage de convaincre une secrétaire qu'il avait recrutée peu de temps après la création de son entreprise en 1927: elle était «blonde, petite, boulotte [...] (et) faisait des fautes d'orthographe ${ }^{14}$ ». L'« enragé » PDG n'avait sans doute pas eu connaissance de l'article "Comment engager votre dactylo secrétaire ${ }^{15}$ ? » que le publicitaire Marcel Nancey avait proposé aux lecteurs de Vendre en 1925. Pour les aider à recruter la leur efficacement, le professionnel a en effet invité ses confrères à faire passer une dictée à chaque candidate potentielle!

Dans la première moitié $\mathrm{du} \mathrm{XX}^{\mathrm{e}}$ siècle, $\mathrm{d}$ 'autres femmes ont peut-être eu des « responsabilités » plus importantes que celles conférées par les métiers de dactylographe ou de secrétaire: en 1917, les Chefs de Publicité observaient dans leur tout premier bulletin que «la guerre» avait ouvert aux femmes «les portes [...] du lieu sévère ${ }^{16}$ » qu'était leur «Conférence ». D'après Marc Martin ${ }^{17}$, entre 1900 et 1939, les «publicitaires [étaient] quelques milliers » : des « portes » auraient été « ouvertes » aux femmes dans ce très parisien et tout petit milieu professionnel, mais combien ont pu les franchir et comment exactement? Pour le moment, difficile à dire. Toujours dans la Rage de

12. Selon Christine Bard, en 1926 (année du premier article rédigé par Paule de Gironde), " $40 \%$ des employés des banques, du commerce [...] » sont des femmes. C. Bard, Les femmes dans la société française au XX $X^{e}$ Siècle, p. 63. 13. À propos de l'usage du substantif»publicitaire ", voir: N. Pelier, "Professionnels de la publicité" ou" publicitaires" ? Comment appeler les hommes et les femmes de l'industrie publicitaire », p. 125-135.

14. M. Bleustein-Blanchet, La rage de convaincre, p. 38.

15. M. Nancey, «La question du personnel: comment engager votre dactylo-secrétaire ?», Vendre, $\mathrm{n}^{\circ} 26$, décembre 1925 , p. 559-564.

16. Les publicitaires, p. 2.

17. M. Martin, Trois siècles de publicité en France, p. 247. Faute de sources, les effectifs sont difficiles à évaluer avec plus de précision. À l'heure actuelle, la profession publicitaire (polymorphe puisqu'elle serait constituée, pour l'Observatoire des Métiers de la Publicité, d'environ 150 métiers différents) peut s'exercer en « agence conseil en communication » et dans deux autres catégories de structures : les « agences médias » et " régies et médias ». Dans les«agences conseils en communication », les «publicitaires » sont aujourd'hui un peu moins de 9700 personnels salariés. 
convaincre, Marcel Bleustein-Blanchet évoque des femmes qui ont eu des responsabilités au sein de Régie-Presse, émanation de Publicis. Il y a eu par exemple une certaine Lucienne Cazavan ${ }^{18}$ (dite Lulu) et Alice, que Bleustein-Blanchet aurait appelée «sa violette de Parme ${ }^{19}$ ». La première a été vice-présidente ; l'autre directrice administrative. Hélas, les archives potentielles de Publicis (de toute façon amputées par un incendie dans les années 1970) n’ont toujours pas été déposées au Musée de la Publicité, où elles devraient, d'après une rumeur (toutefois déjà un peu ancienne), être déposées «prochainement».

Les candidates à l'entrée de la "sévère Conférence » des Chefs de Publicité devaient savoir se «montrer dignes ${ }^{20}$ » de la fameuse ouverture, ont précisé ses dirigeants. Dans la première moitié $\mathrm{du} X \mathrm{X}^{\mathrm{e}}$ siècle, une femme donnait-elle d'elle-même une image "digne » en travaillant dans une agence de publicité ? Vraisemblablement, non : en 1931, Paule de Gironde a relaté dans Vendre qu'une jeune fille à laquelle elle avait voulu présenter sa profession lui avait répondu spontanément et presque avant même que l'explication ait pu commencer :

«Jamais, Madame ! Je suis une artiste et je ne prostitue ni mon crayon ni ma plume ! » ${ }^{21}$

Si les femmes qui ont travaillé dans des agences de publicité n'ont pas donné d'ellesmêmes une très bonne image, leurs homologues de l'autre sexe ont été exposés à des regards négatifs eux aussi. On considère aujourd'hui qu'une certaine publicité appartient à une sorte de patrimoine culturel populaire, mais elle a longtemps été mal considérée et les témoignages de professionnels ayant commencé leur carrière dans le premier tiers du $\mathrm{XX}^{\mathrm{e}}$ siècle évoquent tous les a priori très négatifs de leurs prospects. Revenant sur son expérience professionnelle, dans les années 1920 notamment, Jean Mineur (1902-1985), haute figure de la publicité cinématographique, a déclaré dans son autobiographie :

"Pour les commerçants riches [...], connus pour réaliser de bonnes affaires, faire appel à la "réclame" [aurait été] une attitude déshonorante; on ne [s'abaissait] pas à de telles pratiques, si l'on [était] un homme respectable. [...] Et puis il y avait les femmes, mes ennemies! En général, avec leur esprit pratique, elles ne s'en laissaient pas conter. Elles considéraient [la] réclame comme une $[\ldots]$ escroquerie $[\ldots] »^{22}$

Jusqu'aux années 1970, les publicitaires, qu'ils aient été hommes ou femmes, ont exercé une activité professionnelle mal perçue. Revenant sur ses souvenirs des années 19601970, Michel Coudeyre, cofondateur en 1968 du Club des Directeurs Artistiques (structure destinée à la valorisation de la création publicitaire et de ses auteurs) nous a fait remarquer lors d'une entrevue que nous avons eue avec lui :

« Dans les dîners en ville, on était très, très mal vu si l'on disait qu'on était publicitaire. »

Marcel Bleustein-Blanchet a fait allusion dans ses mémoires, nous l'avons vu, à quelques collaboratrices mais rares semblent être les femmes publicitaires qui ont laissé des commentaires imprimés sur leurs homologues. Entre 1923 et 2010, au moins 25 livres biographiques et autobiographiques relatifs à des hommes publicitaires ont été publiés contre deux seulement côté femmes. Or, parce que la création publicitaire est globalement anonyme (nous l'avons signalé précédemment), les autobiographies et mémoires auxquels nous venons de faire très rapidement allusion constituent un moyen original pour laisser malgré tout un nom dans l'histoire professionnelle de la publicité. Le fait que ces récits soient majoritairement relatifs à des hommes publicitaires et non pas à des femmes donne aux métiers de la publicité, dont les effectifs sont aujourd'hui très largement féminins, l'estampillage d'un milieu « d'hommes ».

\section{Les éventuels ouvrages (auto-)biographiques}

18. M. Bleustein-Blanchet, La rage de convaincre, p. 88 .

19. Ibid., p. 124

20. Les publicitaires, p. 2

21. P. (de) Gironde, «Ce qu'est la technique publicitaire moderne », p. 22

22. J. Mineur, Balzac 00.01 Jean Mineur, le magicien de l'entracte, p. 87. 
À notre connaissance du moins, la seule autobiographie professionnelle de femme publicitaire est celle de l'Américaine Mary Wells Lawrence (née en 1928), haute figure de la pub outre-atlantique dans les années 1970-1980. Co-instigatrice de la journée internationale de la Femme organisée tous les ans le 8 mars, elle a notamment conçu le célèbre slogan Try it, you'll like it («L'essayer, c'est l'adopter ») pour le médicament Alka Seltzer. Son livre, publié en 2002, s'intitule $A$ big life in advertising ${ }^{23} . .$. et n'a pas encore été traduit en français. Côté France, il n'existe pas encore de récit autobiographique signé par une femme publicitaire. On ne connaît qu'un seul ouvrage, «simplement » biographique cette fois, portant sur des professionnelles de la pub : Le beau pour tous [...], publié en $2009^{24}$.

Il porte sur les insolentes fondatrices de l'agence Mafia, créée en 1968. L'une d'elles, Maïmé Arnodin, le «MA» de MAFIA ( FIA " se référant à «Fayolle International Associés») était ingénieure et l'autre, Denise Fayolle, a été championne de patinage artistique à 16 ans. Maïmé Arnodin (1916-2003) est issue d'une famille de banquiers protestants et a effectué ses études au sein de l'École centrale des arts et manufactures. Denise Fayolle (1923-1995), alias « Madame Prisu », a fait des études de philosophie avant de devenir rédactrice pour le magazine Votre beauté et d'intégrer la centrale d'achat de Prisunic dont elle sera licenciée suite à des propos impertinents publiés en 1967 dans Le Nouvel Observateur. Ce sont ces «mafiosies» qui ont créé le fameux «Chouchou» des 3 Suisses. Elles ont également lancé le parfum « Opium » sur fond de polémique. En 1977, Benoîte Groult, future présidente de la Commission pour la féminisation des noms de métiers (fondée en 1984), a refusé que la campagne de presse de la fragrance «pour celles qui s'adonnent à Yves Saint-Laurent» intègre son magazine (Femmes) parce que l'écrivaine estimait qu'aucun individu de sexe féminin n'avait à s'adonner à un homme.

Maïmé Arnodin et Denise Fayolle ont marqué une page dans l'histoire de la profession publicitaire, car Mafia (rachetée en 1985 pour la très coquette somme de 25 millions de francs) a été à la fois la première agence de publicité française créée et dirigée par des femmes et la première intégrant un bureau de style. L'agence n'a pas fait que de la $p u b$ ( $p u b$ par ailleurs perçue comme particulièrement créative et esthétique ${ }^{25}$ ) mais professait ses conseils en conception de produits et s'est donc positionnée bien en amont de la mise sur le marché des produits dont elle assurait la promotion. Le nom tape-à-l'œil de «Mafia » (qui n'est d'ailleurs pas étranger à la visibilité de l'agence ${ }^{26}$ ) a donné un parfum sulfureux à la fameuse entreprise. Cependant, Mafia n'aurait jamais reçu ${ }^{27}$ ces fameuses " sur-commissions ${ }^{28}$ » auxquelles la loi Sapin, dite «loi anti-corruption », a mis un terme en 1993.

Ayant rencontré des exemples de violence symbolique à l'encontre des femmes dans les agences de publicité, la sociologue Laure Gaertner a observé que, dans le sous-groupe

23. M. Wells Lawrence, A big life in advertising, Hardcover, Alfred A. Knopf, 2002, 307 p.

24. S. Chapdelaine de Montvalon, Le beau pour tous. Maîmé Arnodin et Denise Fayolle, l'aventure de deux femmes de style : mode, graphisme, design, Paris, L'iconoclaste, 2009, 261 p.

25. Roman Cieslewicz, par exemple, le graphiste polonais de renommée internationale, a travaillé pour Mafia! 26. Mafia a bien failli s'appeler tristement «SEPT» pour «Style, Esthétique, Publicité et Technique». Le nom, aux allures de chiffre, a été laissé de côté, car il avait déjà été déposé par d'autres entreprises.

27. Dès son année de naissance (1968), Mafia aurait« (adopté) le principe de la facturation à l'heure pour ses honoraires » (du reste " plus élevés que ceux des autres agences à Paris ». S. Chapdelaine de Montvalon, Le beau pour tous..., p. 222.

28. Avec la loi Sapin, le législateur a souhaité clarifier les règles de l'achat d'espace et mettre un terme à la pratique des commissions occultes. Avant 1993, lorsque les agences ou les centrales achetaient de l'espace publicitaire, elles recevaient de la part des supports (c'est-à-dire tel ou tel journal par exemple) une commission d'environ $15 \%$ du montant total de l'achat d'espace à laquelle s'ajoutait très souvent une sur-commission dont le montant n'était bien entendu pas indiqué à l'annonceur. Les agences ou les centrales refacturaient alors l'achat brut aux annonceurs, qui ne pouvaient par conséquent pas connaître le coût réel de l'achat de l'espace de leur publicité. Le recours aux sur-commissions notamment a eu de quoi faire douter de la pertinence des recommandations des agences en ce qui concerne le choix des supports. Aujourd'hui, l'achat d'espace se fait sur la base de tarifs connus pas tous les maillons de la chaîne et le support est tenu d'envoyer directement à l'annonceur l'original de la facture d'achat d'espace. 
des " créatifs $^{29}$ ", catégorie de publicitaires dont l'effectif est à $61 \%$ constitué d'hommes, des « formes de domination masculine » se manifestaient par l'emploi de "stéréotypes culturels de la féminité30 ». Et elle explique qu'il est «attendu [des créatives] qu'elles ne s'offusquent pas » des blagues graveleuses, par exemple, auxquelles elles sont exposées et auxquelles elles sont censées répondre par l'humour ${ }^{31}$. Dans le cadre de ses enquêtes, la chercheuse a pu observer un certain machisme que les fondatrices de Mafia (dont le management n'était toutefois pas réputé pour sa " féminité » ou sa tendresse ${ }^{32}$ ) semblent elles-mêmes avoir subi. Denise Fayolle a en tout cas expliqué que :

«Dans une profession peuplée de "machos" il n' [est...] pas facile de s'imposer. » ${ }^{33}$

C'est par ailleurs ce que raconte avec beaucoup d'humour le film Ce que veulent les femmes, sorti en 2000. Il relate l'histoire de Nick Marshall (incarné par Mel Gibson), créatif-phare d'une agence de publicité américaine. Il espère être nommé directeur de la création et ne se doute pas une seule seconde que ce sera une femme, Darcy McGuire (Helen Hunt) qui aura finalement le poste convoité. Le machisme chronique de Nick l'empêche de concevoir des campagnes pour les cibles féminines mais il s'électrocute accidentellement avec son sèche-cheveux et une nouvelle configuration de son cerveau lui fait entendre ce que pensent les femmes. Il vole alors les idées de Darcy qui les mûrit intérieurement sans se douter du machiavélisme de son collègue. Marshall finit toutefois par tomber amoureux de la brillante publicitaire et les choses vont rentrer dans l'ordre.

Mercedes Erra, le «E » de l'agence BETC (pour Babinet, Erra, Tong-Cuong, co-fondateurs de l'entreprise créée en 1994), validerait sans doute l'affirmation de Denise Fayolle selon laquelle "dans une profession peuplée de "machos" il n' [est...] pas facile de s'imposer ". Réputée chez les professionnels de la publicité notamment pour ses compétences en «construction et gestion de grandes marques", (Lacoste, Petit Bateau, Canal,$+ \ldots$ ), Mercedes Erra a par exemple travaillé autour de la fameuse campagne "aux bébés » d'Évian, et n'est pas étrangère à l'envie de "Faire du ciel le plus bel endroit de la Terre ", promesse d'Air France.

Également connue pour une particularité de son compagnon (" père au foyer ", c'est lui qui s'occupe des enfants!) Mercedes Erra, est décorée de la Légion d'honneur, officier dans l'Ordre national du Mérite et membre de Force Femmes (association pour l'emploi des femmes de plus de 45 ans). Née en Espagne en septembre 1954, elle est arrivée en France à l'âge de six ans. Une vingtaine d'années plus tard, elle a passé et réussi un CAPES de Lettres avant de s'ennuyer dans le professorat et de s'inscrire à HEC Paris puis de devenir assistante Cheffe de publicité dans le groupe Saatchi \& Saatchi. Elle s'est un jour aperçue qu'elle n'avait pas le même salaire que ses associés et s'est mise à militer pour l'instauration de quotas féminins dans les conseils d'administration des entreprises.

29. Les« créatifs » désignent les concepteurs-rédacteurs et directeurs artistiques. Les directeurs de la création sont des créatifs devenus cadres.

30. L. Gaertner, Les créatifs de la publicité entre efficience marchande et idéal d'inventivité : genèse et régulation sociale d'une profession, p. 237.

31. Ibid., p. 338.

32. La biographe de Maïmé Arnodin et Denise Fayolle a par exemple observé que la « rigueur [des fondatrices de Mafia était] stimulante [mais] à condition de tenir». Les deux femmes «[voulaient] que leurs collaboratrices soient séduisantes et chics et [imposaient] pour cela leurs exigences : que le petit haut fleuri soit d'un goût absolument parfait [...]. Maïmé [était] capable de dire à l'une [de ses collaboratrices si celle-ci était] tout ébouriffée, d'aller chez le coiffeur. Téléphone en main, elle [prenait] le rendez-vous à sa place ». S. Chapdelaine de Montvalon, Le beau pour tous..., p. 212.

33. Ibid., p. 192. 
Lorsque l'on cherche à croiser les thèmes " publicité » et «femmes », on pense volontiers en premier lieu à l'image des femmes dans la publicité. Le rapprochement en quelque sorte spontané de ces deux items est lié à l'existence d'une production relativement ancienne et très importante de publicités mettant en scène des femmes, en témoigne par exemple, dans les affiches de la fin du XIX ${ }^{e}$ siècle, la "Chérette", personnage-type féminin, grand conquérant des murs de Paris. Il est probablement lié d'autre part à l'énergie avec laquelle les féministes, réunies en «ligues » ${ }^{34}$ dans les années 1970 puis en « meute $^{35} »$ depuis 2000 , ont dénoncé des campagnes qu'elles jugeaient sexistes.

Le thème « image des femmes dans la publicité » a fait couler beaucoup d'encre mais celui des femmes professionnelles de la publicité bien moins : on ne sait, historiquement nous l'avons vu, quasiment rien sur elles! Dresser une histoire des femmes publicitaires à partir des seules sources imprimées existantes s'avère compliqué : les documents, nous l'avons $\mathrm{vu}$, sont rares et ne permettent guère de sortir d'une histoire des quelques rares notables féminins de la profession.

Une solution consisterait à rencontrer des femmes ayant travaillé en agence pour collecter leurs témoignages. La principale faiblesse de cette solution est de ne pas permettre de remonter à une date très haute. Son principal point positif, et non des moindres, serait de sortir d'une histoire des seules professionnelles ayant, comme Mary Wells Lawrence, les fondatrices de Mafia ou Mercedes Erra, acquis de la notoriété. S'obstiner à étudier les professionnels de la publicité avec une approche "genrée » est important : cela pourrait permettre de renouveler le débat sur l'idée d'après laquelle la publicité serait un vecteur d'images nuisibles à l'égalité des sexes. Mieux savoir qui fait la pub permettrait peut-être de mieux cerner qui elle est. Il s'agit ici d'un véritable challenge. Dans un milieu facilement accusé de recourir au pillage des idées d'autrui, déterminer qui est le père ou la mère d'une campagne est souvent complexe : la création publicitaire est collective et, le plus souvent, distinguer ce que femmes ou hommes ont apporté est difficile, car on ne sait jamais vraiment qui est à l'origine de quoi ${ }^{36}$.

\begin{abstract}
Résumé
Ces dernières années, les études en sciences humaines portant sur la publicité (genre maudit) semblent s'être un peu développées mais, à l'heure actuelle, on dispose encore d'assez peu d'éléments historiques sur les professionnels qui conçoivent, produisent et diffusent la $p u b$. Le manque de connaissances est encore plus flagrant en ce qui concerne les femmes publicitaires. Or, les effectifs des agences de publicité installées en France sont féminins à $62 \%$ environ. Le thème «image des femmes dans la publicité » a fait couler beaucoup d'encre mais pas celui des femmes, presque invisibles, fabricantes de publicités. À l'heure où les études sur le genre et l'égalité professionnelle ont le vent en poupe, cet article aura pour but de présenter quelques femmes publicitaires françaises en exercice de 1925 à nos jours.
\end{abstract}

34. Dans les années 1970, la Ligue du droit des femmes, co-fondée notamment par Simone de Beauvoir, déplorait le caractère "sexiste » de certains messages publicitaires et a demandé (en vain) une extension de la loi Pleven, votée en 1972. Elle devait sanctionner les diffamations commises «envers une personne ou un groupe de personnes à raison de leur origine ou de leur appartenance ou de leur non-appartenance à une ethnie, une nation, ou une religion déterminée ».

35. Fondée en 2000 par Florence Montreynaud, «La meute (des chiennes de garde) » s'est donnée pour objectif de lutter contre la publicité sexiste.

36. On a par exemple pris l'habitude d'attribuer à Philippe Michel (co-fondateur, au début des années 1970, de l'agence CLM) la campagne Myriam, créée en 1981 pour l'afficheur Avenir. En réalité, ce seraient plutôt ses " créatifs ", Joël Le Berre et Pierre Berville qui ont fait naître cette déclinaison dans laquelle (au grand dam des féministes) un mannequin en maillot de bain deux pièces promet d'enlever son top (soutien-gorge) un jour et sa culotte le lendemain. 


\section{Bibliographie}

BARD Christine, Les femmes dans la société française au XX Siècle, Paris, Colin, 2003.

BLEUSTEIN-BlanCHET Marcel, La rage de convaincre, Paris, Robert Laffont, 1970.

Chapdelaine de Montvalon Sophie, Le beau pour tous. Maîmé Arnodin et Denise Fayolle, l'aventure de deux femmes de style: mode, graphisme, design, Paris, L'iconoclaste, 2009.

CHESSEL Marie-Emmanuelle, Publicité: naissance d'une profession (1900-1940), Paris, éditions du CNRS, 1998.

GAERTNER Laure, Les créatifs de la publicité entre efficience marchande et idéal d'inventivité: genèse et régulation sociale d'une profession, thèse de Doctorat, Université Paris X Nanterre, 2008.

GIRONDE (de) Paule, «Ce qu'est la technique publicitaire moderne », Vendre, n 92, juillet 1931, p. 22.

GIRONDE (de) Paule, «Si nous faisions une annonce : comment la construire ? », Vendre, $n^{\circ} 40$, mars 1927, p. 279.

LAFOREST Jeanne, «La joie fait vivre le commerce», La publicité, revue mensuelle des méthodes modernes de vente, $\mathrm{n}^{\circ} 356$, octobre 1937, p. 698-701.

Les publicitaires, bulletin de la CCP (Conférence des Chefs de Publicité), $\mathrm{n}^{\circ} 1$, décembre 1917.

MARTIN Marc, Trois siècles de publicité en France, Paris, Éditions Odile Jacob, 1992.

MiNEUR Jean, Balzac 00.01 Jean Mineur, le magicien de l'entracte, Paris, Plon, 1981.

NANCEY Marcel, «La question du personnel: comment engager votre dactylo secrétaire », Vendre, n² 26, décembre 1925, p. 559-564.

PELIER Nathalie, "Professionnels de la publicité" ou "publicitaires" ? Comment appeler les hommes et les femmes de l'industrie publicitaire ", Cahiers de RECITS, n 9, 2013, p. 125-135.

POUILlARD Véronique, La publicité en Belgique 1850-1975, des courtiers aux agences internationales, Bruxelles, Académie Royale de Belgique, 2005.

Wells Lawrence Mary, A big life in advertising, Hardcover, Alfred A. Knopf, 2002. 


\title{
L'explication du geste technique, la transmission des savoir- faire : l'indicible et la communication non verbale à travers l'exemple des monographies d'entreprises du chantier 1810 au MNATP ( musée national des arts et tradition populaires)
}

\author{
Bénédicte ROLLAND-VILLEMOT \\ Conservateur du patrimoine \\ Institut du patrimoine, \\ département des restaurateurs, \\ chargée de mission auprès du directeur des études \\ Extrait de : Xavier LAFON, Les langues savantes, Paris, \\ Édition électronique du CTHS (Actes des congrès des sociétés historiques et scientifiques), 2017. \\ Cet article a été validé par le comité de lecture des Éditions du CTHS dans le cadre de la publication \\ des actes du $139^{\mathrm{e}}$ Congrès national des sociétés historiques et scientifiques tenu à Nîmes en 2014 . \\ «Sculpte, lime, cisèle, que ton rêve flottant se scelle dans le bloc résistant » ${ }^{1}$ \\ Théophile Gautier, L'art \\ Le geste, l'action se verbalise : on la décrit par des verbes à l'infinitif ou à l'impératif. Ce \\ langage verbal est-il suffisant pour décrire, comprendre et transmettre le geste et \\ l'action ? Comment on apprend et on transmet les « ficelles » du métier ?
}

\section{Définition du geste technique}

Dans le Petit Robert, le geste technique est défini comme "mouvement du corps (principalement des bras, des mains, de la tête) volontaire ou involontaire révélant un état psychologique ou visant à exécuter quelque chose, simple mouvement expressif ou caractéristique, acte ou action ». "Acte, action » est ainsi présenté dans le Larousse :

«Manière de mouvoir, le corps, les membres et, en particulier, de mouvoir les mains dans un but de préhension, de manipulation. Métier qui demande une précision dans le geste. Le geste dit technique est plus spécifique, lié à une pratique, ou un travail particulier. »

Le geste technique est fondateur de l'homme. Comment décrire le geste technique ou plutôt l'action? Comme l'affirme Leroi-Gourhan "L'outil n'est réellement que dans le geste qui le rend techniquement efficace $»^{2}$. Comment se transmettent les informations? Les savoir-faire? Le savoir-faire développé par l'homme s'exprime à travers les mains et dans la symbiose entre le geste et un outil. Le savoir-faire doit être rapproché ici de la mètis des Grecs, cette intelligence rusée disséquée par Marcel Détienne et Jean-Pierre Vernant. 
Ce qui dans la technique, cet «acte traditionnel efficace", ne se dit ni ne se montre ni donc bien souvent ne se transmet explicitement mais se sent, s'incorpore, se vit au jour le jour, s'exprime dans le milligramme du cuisinier, le «nez » du parfumeur, la dextérité du tronçonneur, ou l'oreille du fondeur de cloches et que l'on nommera parfois le « tour de main », le « vice » et, le plus souvent, le «savoir-faire».

Ce «presque rien », pour paraphraser Jankélévitch ${ }^{3}$, qui aux dires des gens de métier ferait l'efficacité du geste technique, c'est le typographe dont le savoir-faire réside dans toute une série de gestes difficilement décelables car situés au niveau de "l'épiderme et des nerfs sensitifs... doigts passés sur la ligne composée pour apprécier sa tension... appréciation à la main $\mathrm{du}$ poids $\mathrm{du}$ plomb qui permet d'identifier le corps d'un caractère ${ }^{4}$. C'est le faïencier qui doit être en mesure de s'arranger avec son propre corps, savoir régler le jeu des postures et des mouvements, "régulation qui peu à peu devient une seconde nature indicible». Blandine Brill, a démontré les difficultés de méthodes pour décrire le geste à cause de l'incroyable diversité du geste technique ${ }^{5}$.

Y a-t-il un moyen de travailler sur l'apprentissage du geste technique? De fait, c'est l'action et son efficacité qui sont importantes plus que le geste qui est très variable. On apprend à réaliser une action. Par exemple, donner la bonne vitesse de frappe à un marteau. Le geste dépend des contraintes de la tâche à effectuer.

Est-ce que les disciplines comme la tracéologie peuvent apporter leur contribution pour un décryptage du geste technique? Cette discipline archéologique classe et étudie les différents types d'empreintes ou de marques encore visibles sur les matières travaillées. Elle peut permettre d'identifier les outils utilisés, de restituer les diverses étapes de fabrication et les savoir-faire employés et les éventuelles marques d'usages et d'usures. Parfois, elle peut aussi déterminer la durée de l'emploi d'un objet et la manière dont il était utilisé.

Cerner les difficultés méthodologiques et pratiques pour étudier les gestes techniques et les traces qu'ils peuvent laisser sur les objets ou alors comment, en étudiant des outils, on peut en déduire des gestes.

Comment identifier, décrire pour le reproduire, un objet qui par sa nature même se dérobe à l'investigation comme à l'explicitation? L'explication du geste, sa description, doivent emprunter une méthodologie rigoureuse et utiliser plusieurs techniques: le dessin, le dessin technique, la photographie, le reportage, la description textuelle. ( Fig. 1) Mais peut-on le transmettre? Est-il reproductible? comme l'écrit Leibniz :

«Il n'y a point d'art mécanique si petit et si méprisable, qui ne puisse fournir quelques observations ou considérations remarquables et toutes les professions ou vocations ont certaines adresses ingénieuses dont il n'est pas aisé de s'aviser et qui néanmoins peuvent servir à des conséquences bien plus élevées. »"

Les critiques de la parcellisation des tâches qui se développent au cours des années soixante 70 avec les nouvelles formes d'organisation du travail : la multiplication des travaux des ergonomes énumérant les situations où les agents contreviennent aux procédures prescrites, le plus souvent au bénéfice du déroulement correct du processus de travail, les analyses des formes de résistance à la taylorisation constituent autant de repères dans l'attention nouvelle apportée aux savoirs ouvriers. De fait, le geste n'est pas incarné dans l'objet de l'ère industrielle, il ne laisse pas de trace. C'est la marque de fabrique qui identifie l'objet.

Il faut alors observer et manipuler pour comprendre le fonctionnement de l'objet. Il faut analyser la capacité d'affordance de l'objet, ou plus exactement sa capacité à suggérer sa propre utilisation. Comment trouver des dispositifs pour décrire par des mots ces gestes

3. Le Je-ne-sais-quoi et le presque-rien, Paris, PUF, 1957.

4. Carré, Tiévant, 1990, p. 70-71.

5. B. Bril, «Description du geste technique : Quelles méthodes?», Techniques \& Culture [En ligne], 54-55 | 2010, mis en ligne le 30 juin 2013, consulté le 17 juin 2014. URL : http: / / tc.revues.org/ 5001

6. G. Wilhelm Leibniz, Discours touchant la méthode de la certitude et l'art d'inventer, Berlin, 1890, p. 174. 
techniques, soit pour la conservation du patrimoine technique soit pour leur valorisation et leur transmission?

Il faut essayer de comprendre ce qu'est un geste technique : se pose alors le problème de son acquisition et de son apprentissage. Le geste est une cinématique qui s'exprime par le mouvement et par l'action. La parole est-elle nécessaire à la transmission des savoir-faire et des gestes techniques? Ils ne se transmettent pas par la parole : qu'exprime-t-on du mouvement à partir du langage?

\section{Le problème de la répétitivité du geste pour être efficace?}

Pour décrire le geste, il existe des descriptions comportementales et des descriptions fonctionnelles: un geste technique est un geste efficace sur la matière. Leroi-Gourhan l'explique en termes d'action et non de mouvement :

«Nous montrerons que les capacités transformatives des matériaux jouent un rôle fondamental dans leur sélection et leur utilisation et que, par ailleurs, toute transformation suppose des actions calculées qui sont, par nature, relationnelles. Nous affirmons que la sélection et la manipulation technique des matériaux vont de pair avec une empathie, une sorte d'intersubjectivité, qui permet la reconnaissance du potentiel de certains matériaux comme étant capables de répondre à des attentes essentielles et permettant de parvenir à des résultats spécifiques. ${ }^{7}$

\section{Le chantier 1810 : la méthode de la monographie d'entreprise artisanale}

Les chantiers intellectuels lancés à la demande de l'État français constituent encore aujourd'hui une source majeure pour la recherche en ethnologie. Le chantier 1810, dirigé par Marcel Maget, illustre la conception de la démarche empirique fondée sur l'enquête de terrain telle que le musée avait pu en organiser dès 1939. L'ethnologie folklorique pendant le régime de Vichy confirme le rôle déterminant que peuvent jouer les enquêtes dans le domaine de la recherche scientifique.

Dans les années 20, la réflexion sur les rapports entre l'artisanat et l'art populaire est présente dans la démarche des folkloristes. L'ethnologie, science sociale qui se développe à l'époque, emploie la méthode descriptive qu'est la monographie pour viser à l'exhaustivité. Marcel Mauss, dans son Manuel d'ethnographie, écrit :

«On collectionnera tous les produits fabriqués; on étudiera tous les moments de la fabrication des machines. ${ }^{8}$

La méthode de la monographie qui vise à traiter son objet dans sa totalité et veut aboutir à la synthèse est alors élaborée. La monographie est un genre ethnologique consistant en l'analyse la plus complète possible d'un groupe humain, d'une institution ou d'un fait social. Elle désigne à la fois une méthode spécifique d'enquête et une forme d'exposition des résultats de la recherche. Elle demande une observation directe et prolongée des faits. Le premier à utiliser cette méthode fut Frédéric Le Play en 1826. Puis, dans les années 20, avec le rejet de l'ethnologie de cabinet, l'ethnologie «exotique » développe cette méthode. En 1922, Malinowski écrit une monographie sur les Tobriandais. Cet effort de méthodologie part de l'enseignement de Marcel Mauss à l'Institut d'ethnologie entre 1928 et 1939. Marcel Maget va s'en inspirer pour définir une méthode spécifique propre à l'ethnographie. Entre 1943 et 1944, il est chargé de l'organisation d'un service de recherche au MNATP. Dans un texte du 18 octobre 1943 qui s'intitule " ethnographie folklorique et ethnologie », il décrit cette méthode :

7. Leroi-Gourhan, Le geste et la parole, p. 65.

8. M. Mauss, Manuel d'ethnographie, p. 28. 
"Chacun des objets étudiés ou recueillis dans les missions d'ethnographie entreprises sous la direction du musée de l'Homme, fait l'objet d'une description complète où apparaissent non seulement à côté de son nom : ses caractéristiques matérielles, dimensions, matières... mais aussi les procédés de fabrication et ses caractères économiques sociaux et idéologiques. »

Les monographies permettent des études comparatives pour établir des rapports, des filiations ou des survivances, des traits culturels archaïques qui font appel aux notions de caractères et de transmissions populaires. Les monographies, en établissant un simple constat doivent également fournir le substrat d'une analyse comparative et typologique. Or, c'est l'artisanat, dans le contexte de la France rurale de l'entre-deux-guerres, qui apparaît comme le meilleur objet d'étude pour faire surgir ces survivances, pour les étudier, les conserver et les transmettre afin de développer un art populaire vivant. L'exposition internationale de Paris en 1937 était destinée entre autres, selon le commissaire général de l'Exposition, Edmond Labbé, à développer un thème particulier, les arts et les techniques. Les diverses appréciations de la nature du rapport entre arts et techniques vont donner lieu à différentes manifestations rattachées soit aux métiers d'art (les objets de luxe), soit à une production essentiellement régionale: un centre des métiers, un centre rural, un centre régional pouvaient être visités lors de cette exposition. Le centre régional fut l'un des clous de l'exposition. Labbé écrit :

«J'ai pour ma part confiance dans l'avenir du régionalisme, dans l'avenir de son aspect technique d'abord, c'est-à-dire de l'artisanat, j'ai entendu prouver que l'usine, faite à la mesure de la machine, ne supplantera pas définitivement l'atelier, fait à la mesure de l'homme et que l'évolution des procédés de productions n'a frappé qu'à mort les formes inférieures du travail de l'artisanat. [...] Dans cette France, notre domaine, comment constituer nos collections? Les produits et les instruments de la civilisation industrielle n'étaient pas pour nous. Dans un monde en pleine transformation, il fallait atteindre les témoins épargnés d'une civilisation artisanale arrivée à son stade ultime, mais encore florissante un siècle et demi plus tôt. »

Voilà le programme défini par Georges-Henri Rivière. La méthode monographique paraît alors la plus appropriée pour étudier la culture matérielle et plus particulièrement les techniques. Accompagnées d'enquêtes-collectes destinées à enrichir les collections du futur musée, des monographies furent expérimentées en Bretagne et en Sologne.

\section{Description de la méthode employée pour ce chantier}

Ce troisième chantier (dit «1810») est organisé en relation avec le ministère de l'Agriculture et la Corporation nationale paysanne. Il concerne "les arts et traditions populaires de la paysannerie » et utilise la méthode monographique. La monographie d'entreprise artisanale a été mise au point par Marcel Maget et enseignée dans le cours d'ethnographie française à l'École du Louvre à partir de 1941 dans le cadre du cours organique consacré à l'ethnologie de la France mis en place par Georges-Henri Rivière en 1939. Entre 1942 et 1946, sous la direction de Marcel Maget, alors directeur du service de la civilisation paysanne au sein de la Corporation paysanne, 63 enquêtes ont eu lieu, réparties dans la France entière mais plutôt concentrées dans la France de l'Ouest. Les enquêteurs doivent utiliser la méthode monographique définie par Marcel Maget. Ce chantier 1810 est celui qui développe le plus la méthode de l'enquête intensive. Maget va développer tout une méthodologie de la description d'objets et entreprises artisanales dans différents textes dont l'instruction provisoire pour la constitution d'une collection d'ethnographie française, la monographie d'objets.

Ce texte conduit à la mise au point d'une fiche «d'objet folklorique» qui doit être la méthode employée pour la description des objets entrant au musée. Dans un ordre de service du 9 mai 1941 à destination du personnel scientifique du MNATP, Maget rédige des instructions préliminaires à une fiche d'objet folklorique. Il développe cette notion de fiche d'objets dans une instruction du 27 octobre 1943 " dédiée au personnel et aux amis du MNATP, aux équipiers des chantiers du service des chantiers intellectuels et artistiques et 
aux élèves du cours d'histoire des arts et traditions populaires de l'école du Louvre ». Dans la préface, il écrit "les présentes instructions ont été élaborées au Musée de l'Homme (dès 1929), puis au MNATP ».

Selon cette instruction, cette fiche d'objet a deux buts : décrire les objets sur le terrain de l'enquête et servir à la rédaction de la fiche définitive d'objet quand celui entre dans les collections du musée. Cette fiche est donc une monographie d'objet qui doit suivre une méthode rigoureuse : dénomination exacte de l'objet, description physique, techniques de fabrication, techniques d'utilisation, histoire du spécimen et du type ainsi que, si possible, des comparaisons. Les équipiers des différents chantiers doivent donc utiliser cette méthode pour décrire les objets ou machines qu'ils vont trouver chez les artisans. La monographie d'entreprise comprend donc des monographies d'objets ou fiches descriptives, ce qui permet de décrire une chaîne de fabrication avec précision. Il écrit également une note critique sur les outils de l'enquête. Il va également s'intéresser au rôle de l'image comme outil de connaissance et de description dans l'enquête, ce sont des «suggestions concernant l'emploi de la photo et du cinéma dans la recherche en ethnographie paysanne ». Dans ce texte, il insiste sur le rôle important de l'image comme outil de documentation et de description indispensable à l'enquête de terrain. Dans le texte du 18 octobre 1943 déjà cité, Maget conclut :

« Reprenant la tradition des encyclopédistes du XVIII siècle, et étendant à l'ethnographie les méthodes qui peu à peu s'élaborent et confirment leur efficacité en ethnographie, G.-H. Rivière a voulu que l'on entreprenne une description approfondie des techniques artisanales qu'il est encore permis d'observer sur le territoire métropolitain. »

Mais plus loin, il ajoute :

« Nous avons insisté sur la méthode monographique, mais il ne faudrait pas en conclure de la considérer comme le seul moyen de mener une enquête intensive. Elle ne se suffit que si les points d'observations sont suffisamment rapprochés et étudiés dans un laps de temps suffisamment court pour rendre les comparaisons valables. »

Cette méthode de la monographie d'entreprise artisanale doit préciser dans le cadre de chaque enquête, pour chaque entreprise ou atelier artisanal, le milieu géographique, les matières premières, les produits fabriqués, le rythme de production, les gestes employés, l'action technique, le contrat social et économique, une biographie des artisans, un relevé des coutumes ainsi qu'un historique du métier. Ces éléments textuels, selon la méthode monographique, doivent être complétés par des dessins, des relevés, des croquis. Chaque enquête devait suivre la même méthode: recherche sur le terrain, préparation et élaboration d'un journal de route, chiffres, croquis, dessins mis au net, éventuellement accompagnés de photographies.

\section{Organisation du chantier}

Dans une note du 3 juin 1943 consacrée aux trois chantiers, G.-H. Rivière et Maget expliquent leurs objectifs en créant ces chantiers :

« Des circonstances sans précédent dans l'histoire du monde agissent depuis un siècle sur le développement de la civilisation occidentale. La révolution industrielle bouleverse les techniques et l'économie et sape les bases millénaires de l'ancienne société agricole et artisanale. »

Les buts du chantier 1810 sont ainsi définis :

«a) accomplir une recherche scientifique aboutissant à une documentation mise à la disposition du secrétariat général des beaux-arts et ouverte à tous les spécialistes intéressés ; b) élaborer une doctrine et un programme de conservation et de rénovation d'éléments du folklore à transmettre ou à incorporer dans la société nouvelle ; c) former un personnel susceptible de collaborer à ces diverses activités avec le concours des artistes repérés au cours de la recherche. » 
Ce chantier avait donc initialement deux buts: une recherche scientifique pour documenter les artisanats traditionnels afin de les transmettre; la documentation et la transmission des savoir-faire. Les équipiers ont un profil «d'ethnographes, de techniciens ou d'artistes ». Chaque équipier obéit à un plan de travail dont l'exécution est contrôlée mensuellement par un compte-rendu. Ils doivent tenir un journal de route. Des rapports annuels des travaux sont rédigés pour le secrétariat général à l'agriculture, accompagnés d'un programme pour l'année suivante.

\section{Les enquêteurs}

Lors de sa création en novembre 1942, le chantier devait embaucher 20 équipiers. Mais par un arrêté du 19 mai 1943, il n'est prévu que 14 équipiers. En réalité, par manque de crédits, le chantier ne pourra employer que 13 équipiers (12 et Maget). En 1945, un enquêteur coûtait $6500 \mathrm{~F}$ par mois. Dans les rapports remis annuellement, on peut étudier les évolutions et les difficultés d'organisation qu'a connues ce chantier. Ainsi, à partir de mars 1943, à cause de la mise en place de la ligne de démarcation, les déplacements des équipiers sont difficiles. Dans le programme pour l'année 1945-1946, pour tenir compte des désordres de la Libération, des modifications sont prévues :

«La période de transition durant laquelle la raréfaction des moyens de transport empêchant le travail d'enquêtes importantes sera utilisée pour l'étude de certains artisanats de Paris. »

C'est dans ce cadre que se déroule l'enquête au sein de l'entreprise Hermès à Paris. L'étude du corpus disponible pour le chantier 1810 aux archives du MNATP montre une importante diversité : vanniers de Brière, potiers de la Borne, fondeurs de Villedieu, artisanats du bois, tonneliers, sabotiers.

Ces 63 monographies d'entreprises artisanales ont aussi pour but de décrire les gestes, l'action technique par des méthodes scientifiques. Les enquêteurs utilisent des méthodes scientifiques pour essayer de comprendre ce qui se passe dans l'acte technique. Ils décomposent les phases de fabrication pour comprendre la tendance technique. Ils chronomètrent les phases de fabrication, se renseignent sur les rythmes de travail, la durée de la journée de travail, le nombre de pièces produites.

Ils étudient les lieux de travail, l'organisation spatiale de l'atelier ou des ateliers. Ils décrivent avec une grande précision l'outillage : ils rédigent une fiche descriptive des outils et machines en expliquant leur fonctionnement. Ils demandent aussi des renseignements sur la division du travail. Dans chaque monographie, sont précisés le nombre d'apprentis, le nombre d'employés, le nombre et la qualité des collaborateurs. Qui fait quoi ? Quelles étapes décisives de la fabrication sont effectuées et par qui ?

En analysant toutes ces données, il est possible, par défaut et par effet de miroir, de comprendre ou du moins d'entrevoir des gestes techniques. Ainsi, dans la monographie la plus complète de cette série, l'étude de l'atelier de potier de Saint-Jean-la-poterie en Bretagne, Dan Lallier étudie minutieusement la position des potières. Dans son carnet il note: " position de la potière sur la tourneuse ». Il dessine la potière et accompagne ces croquis par un texte détaillé :

«Pour le montage, la potière se courbe vers la girelle, mouille à nouveau ses mains, prend la masse à deux mains, les extrémités des doigts joints prennent la masse pressée par les 2 paumes et les doigts s'élèvent. »"

Ensuite, il décrit l'opération du creusage :

«Le creusage au pouce : le pouce de la main droite s'enfonce dans le centre de la masse. La main gauche maintient l'argile latéralement. Il prend la masse à 2 mains, paumes plaquées contre l'argile, la presse et la fait légèrement monter. » 
Toutes ces descriptions textuelles sont accompagnées d'une iconographie riche et méthodique, notamment le dessin technique des différentes séquences du travail. L'enquêteur segmente les différentes opérations et les dessine de façon méthodique en numérotant les phases avant de les photographier.

Les dessins sont très codifiés. Ils présentent d'abord une vue d'ensemble de l'atelier, de l'artisan devant son métier. Puis l'enquêteur porte son attention sur les mains. Dans l'enquête sur les potières de Saint-Jean-la-poterie, Dan Lallier dessine les mains en train de monter un pot. Il reprend par le dessin les principales opérations en notant avec précision les positions des mains à l'extérieur et à l'intérieur, en donnant pour cette dernière la position en pointillé.

L'étude des postures au travail (fig. 2) renseigne aussi sur les gestes et l'action technique. Par des dessins successifs, presque une image par image, ces monographies analysent pour chaque métier les postures de bases qui sont synthétisées par des diagrammes corporels. Ce travail séquentiel, l'objectif de ce chantier, permet des études comparatives. Pour essayer d'appréhender les gestes efficaces et efficients, les enquêteurs demandent aux artisans comment ils ont appris leur métier et auprès de qui. La réponse est presque toujours la même: ils ont appris sur le tas chez un artisan, en commençant comme apprenti, puis en progressant petit à petit. Jules Coty, potier dans le Calvados (Fig. 3), déclare :

«Il faut bien un an au four pour former un apprenti et il faut bien 7 ou 8 ans pour être ouvrier et tout connaître; quand on sait tourner, on tourne tout. » ${ }^{10}$

Les enquêteurs se posent aussi la question de savoir comment on devient expert et comment on acquiert ces compétences. Comment déceler cette empathie avec les matériaux?

Pour essayer de comprendre la bonne tenue des pots à la cuisson de la Veuve Marie Noury à Saint-Jean-la-poterie, l'enquêteur fait des prélèvements de la terre crue et cuite pour analyse par le laboratoire de la manufacture de Sèvres.

«Ce n'est donc pas à la nature spéciale des matières premières qu'il faut attribuer la bonne tenue au feu de la poterie.»

C'est donc habilité de la potière. Or cette habilité, cette expertise sont difficiles à analyser et à expliquer. D'après Leroi-Gourhan, «c'est la fonction qui fait l'outil ». Grâce à la précision des fiches descriptives des outils employés et des ustensiles produits, l'étude d'un atelier de chaudronnerie dans le Tarn permet de déduire le degré "d'affordance " des objets et donc d'analyser les gestes techniques efficients (fig. 4). L'affordance est la capacité de l'objet à suggérer sa propre utilisation. L'outil est conditionné à l'origine par le geste à faire et il conditionne à son tour les gestes à venir. L'outil crée le mouvement ${ }^{11}$.

Cet ensemble de monographies est bien un alphabet du geste technique. Mais cet alphabet ne s'exprime pas par le langage parlé ou écrit. C'est par la communication visuelle (dessins, photographies,... ) que se comprend le geste. Son apprentissage et sa transmission au sein des ateliers se font aussi par une communication visuelle. C'est par immersion et en regardant que l'apprenti comprend ce qu'il doit faire. Aujourd'hui c'est par le film, l'enregistrement vidéo, que l'on décrit mieux le geste technique et l'action technique. La popularité des émissions et des vidéos consacrées à la cuisine et à la réalisation de recettes montre bien que l'image joue un grand rôle dans l'apprentissage des techniques. 


\begin{abstract}
Résumé
Le geste technique est fondateur de l'homme. Comment se transmettent les informations ? Les savoir-faire? Le corps, «le premier et le plus naturel instrument de l'homme » selon Marcel Mauss ${ }^{12}$. L'association du geste et de l'objet est une interface entre l'homme et la technique. Dans la technique, cet «acte traditionnel efficace», ne se dit ni ne se montre ni donc se transmet par le langage mais se sent, s'incorpore, se vit au jour le jour, dans l'atelier. Comment trouver des dispositifs pour décrire par des mots ces gestes techniques soit pour la conservation du patrimoine technique soit pour leur valorisation et leur transmission? L'étude des monographies d'entreprises artisanales effectuées dans le cadre du chantier intellectuel 1810 par l'équipe du Musée national des arts et tradition populaire (MNATP) entre 1940 et 1946 apporte des réponses à la question: comment expliquer le geste technique. Le geste technique est fondateur de l'homme. Comment se transmettent les informations ? Les savoir-faire ? Le savoir-faire développé par l'homme s'exprime à travers les mains et dans la symbiose entre le geste, et un outil. Le corps, «le premier et le plus naturel instrument de l'homme » selon Marcel Mauss (référence). L'association du geste et de l'objet est une interface entre l'homme et la technique. Le savoir-faire est l'ensemble des compétences acquises, incorporées, transmises, qui se manifestent dans l'acte technique. Dans une période de rupture, il est impossible de formaliser par le langage les savoir-faire pour les incorporer dans les nouvelles technologies que sont les systèmes experts. Dans la technique, cet « acte traditionnel efficace », ne se dit ni ne se montre ni donc se transmet par le langage mais se sent, s'incorpore, se vit au jour le jour, dans l'atelier. Comment trouver des dispositifs pour décrire par des mots ces gestes techniques soit pour la conservation du patrimoine technique, soit pour leur valorisation et leur transmission?
\end{abstract}

\title{
Sources
}

Archives nationales : archives ATP série 2013277

\section{Bibliographie}

Leroi-Gourhan André, Le Geste et la Parole, 1. : Technique et langage, 2.: Mémoire et les Rythmes, Paris, Albin Michel, 1964-1965.

BRIL Blandine, «Description du geste technique: Quelles méthodes ? », Techniques $\mathcal{E}$ Culture [En ligne], 54-55 | 2010, mis en ligne le 30 juin 2013, consulté le 17 juin 2014.

URL : http:/ / tc.revues.org/5001

MAUSS Marcel, Manuel d'ethnographie, Paris, Éditions sociales, 1926, 264 p.

Repris de l'édition électronique de J.-M. TREMBLAY

http: / / classiques.uqac.ca/classiques/mauss_marcel/manuel_ethnographie/manuel_eth nographie.pdf

ROBERT Jean-François, Rêver l'outil, Paris, éditions Cabédita, 1995, 188 p.

12. Mauss M., « Les techniques du corps », Sociologie et anthropologie, Paris, PUF, 1950, p. 372. 


\section{Illustrations}

Figure 1: Atelier de Monsieur Plaisance à Noron-la-Poterie. Dessin au crayon de Dan Lallier (AN série 2013277 / 118). CBénédicte Rolland-Villemot.

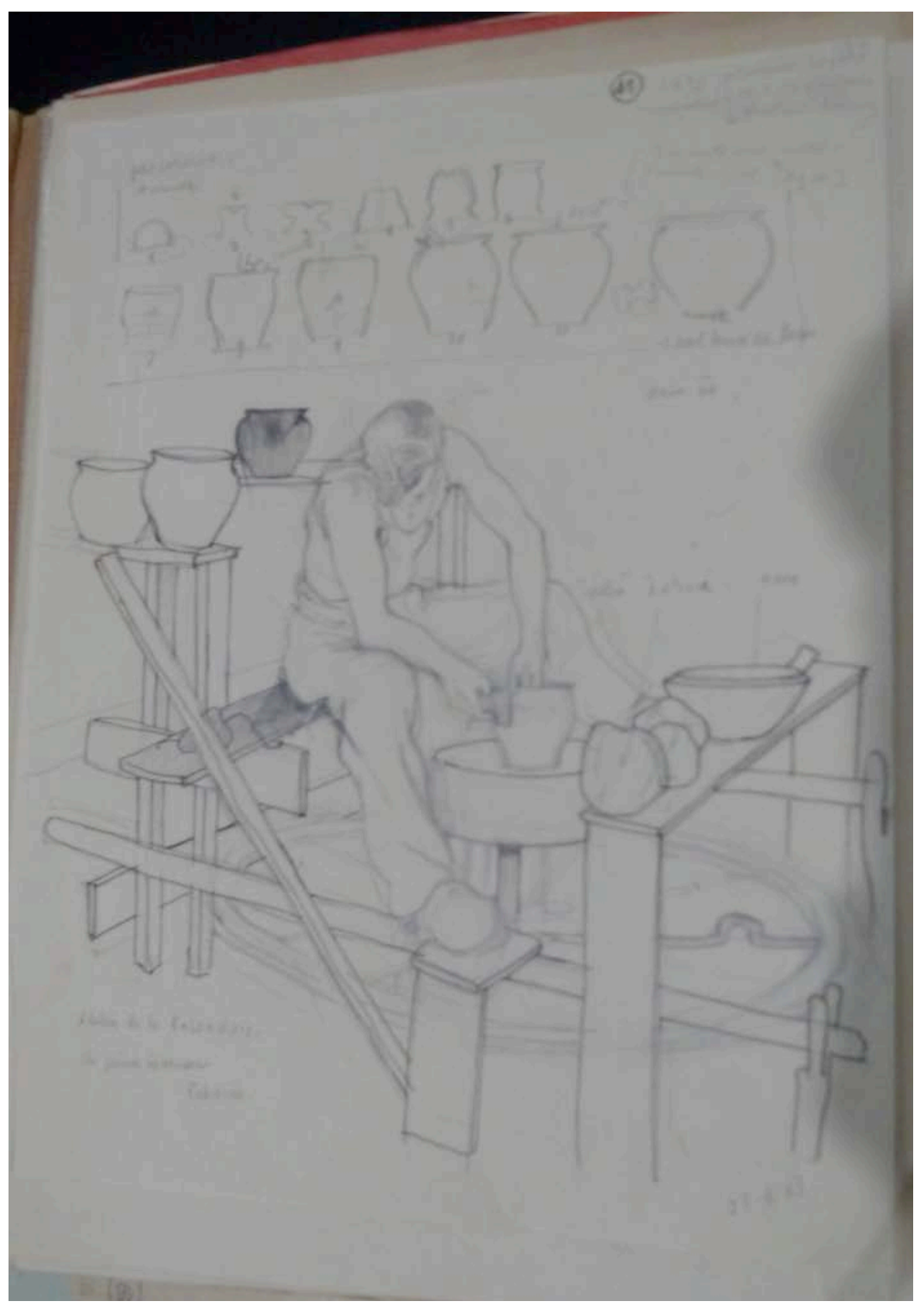


Figure 2: Noron-la-Poterie, dessin technique sur calque, Dan Lallier (AN série 2013277/118) usage de la tournette? et étude de la posture du corps au travail. cBénédicte Rolland-Villemot.

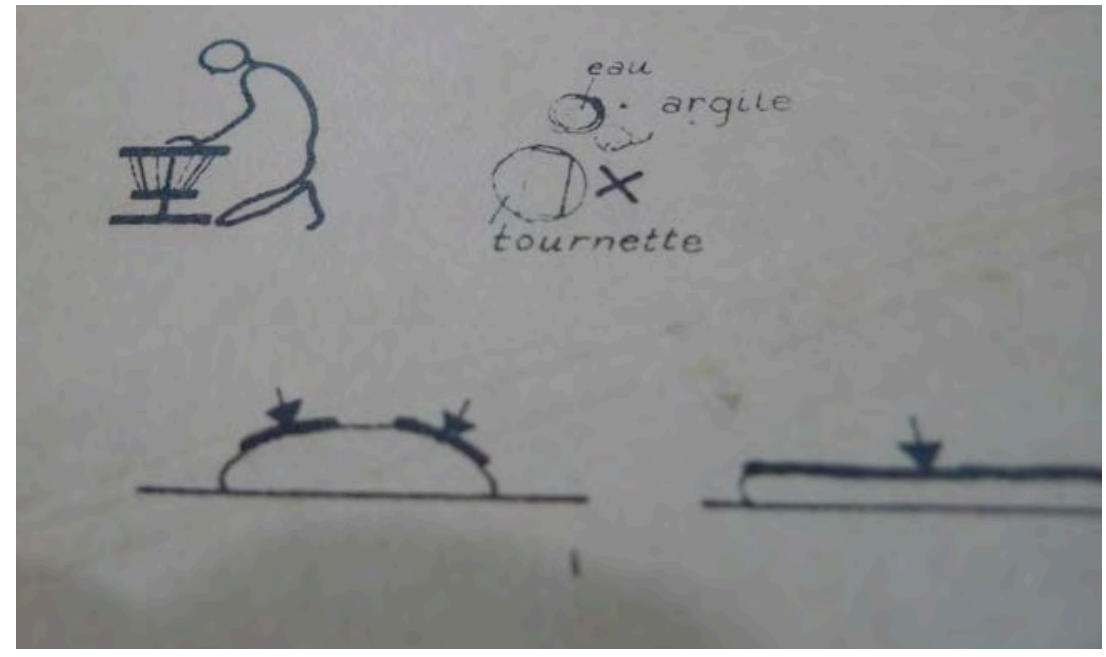

Figure 3 : mains de Jules Coty, potier dans le Calvados, en train de monter un pot (AN série 2013277 / 116). Dessin au crayon de Dan Lallier. (CBénédicte Rolland-Villemot.

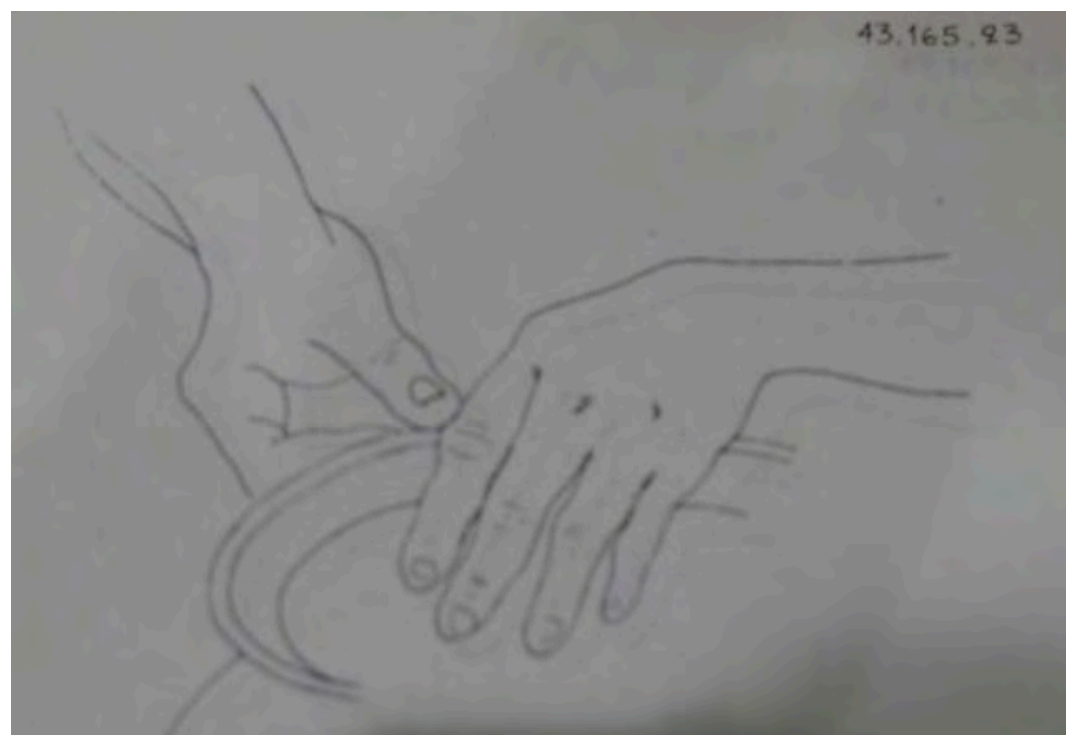


Figure 4: dessins et relevés techniques d'ustensiles de cuisine en cuivre chez un dinandier dans le Tarn. Dessin de Guy Moinet. OBénédicte Rolland-Villemot.

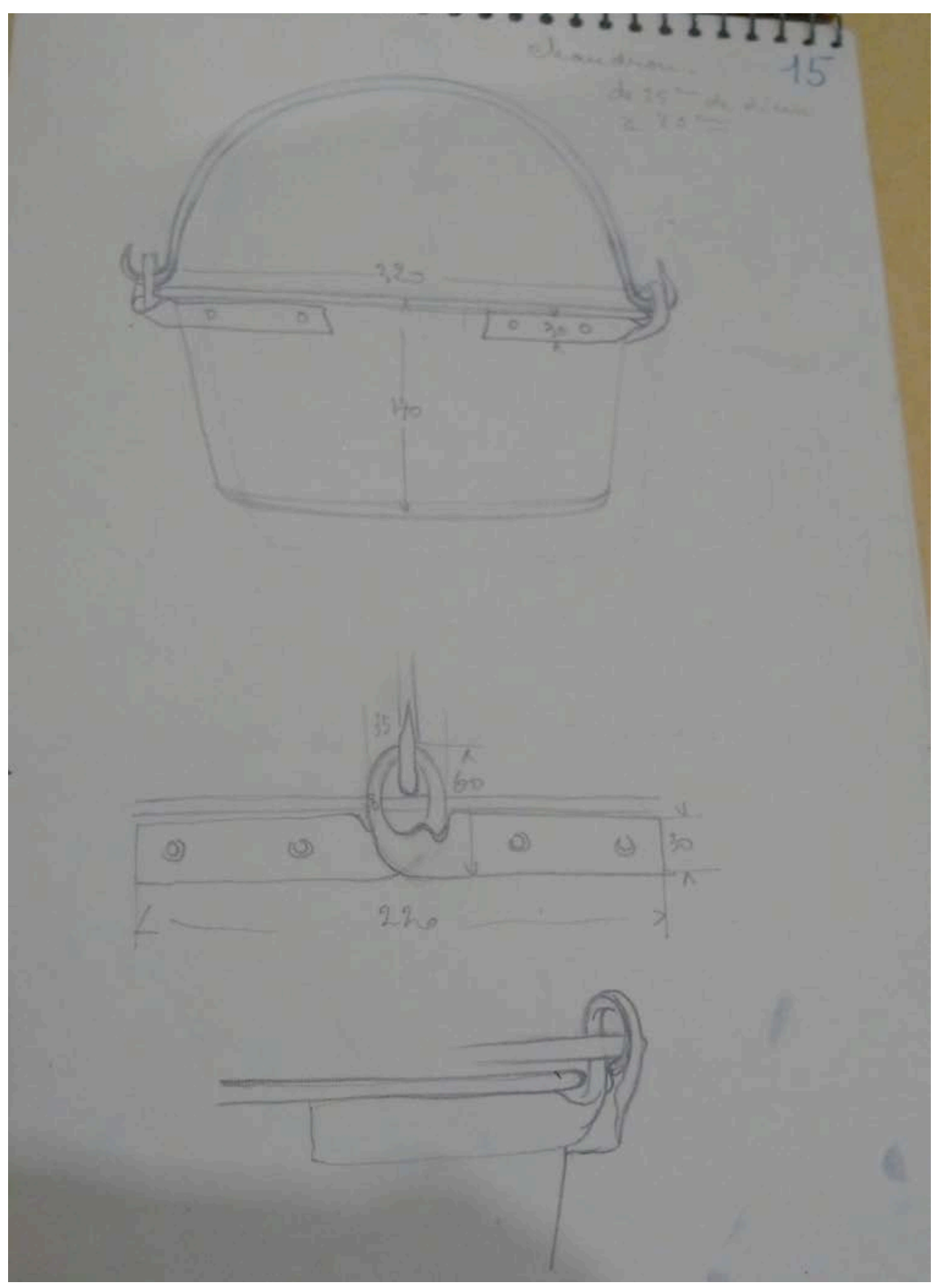

